Florida International University FIU Digital Commons

FIU Electronic Theses and Dissertations

University Graduate School

9-20-2011

\title{
Carbon Nanotube Based Systems for High Energy Efficient Applications
}

Indranil Lahiri

Florida International University, ilahi001@fiu.edu

DOI: $10.25148 /$ etd.FI11120614

Follow this and additional works at: https://digitalcommons.fiu.edu/etd

\section{Recommended Citation}

Lahiri, Indranil, "Carbon Nanotube Based Systems for High Energy Efficient Applications" (2011). FIU Electronic Theses and Dissertations. 508.

https://digitalcommons.fiu.edu/etd/508

This work is brought to you for free and open access by the University Graduate School at FIU Digital Commons. It has been accepted for inclusion in FIU Electronic Theses and Dissertations by an authorized administrator of FIU Digital Commons. For more information, please contact dcc@fiu.edu. 


\section{FLORIDA INTERNATIONAL UNIVERSITY}

Miami, Florida

\section{CARBON NANOTUBE BASED SYSTEMS FOR HIGH ENERGY EFFICIENT APPLICATIONS}

A dissertation submitted in partial fulfillment of the requirements for the degree of DOCTOR OF PHILOSOPHY

in

MATERIALS SCIENCE AND ENGINEERING

by

Indranil Lahiri 


\section{To: Dean Amir Mirmiran}

College of Engineering and Computing

This dissertation, written by Indranil Lahiri, and entitled Carbon Nanotube Based Systems for High Energy Efficient Applications, having been approved in respect to style and intellectual content, is referred to you for judgment.

We have read this dissertation and recommend that it be approved.

$\begin{array}{r}\text { Arvind Agarwal } \\ \hline \text { Norman D.H. Munroe } \\ \hline \text { WonBong Choi, Major Professor }\end{array}$

Date of Defense: September 20, 2011

The dissertation of Indranil Lahiri is approved.

Dean Amir Mirmiran

College of Engineering and Computing

Dean Lakshmi N. Reddi

University Graduate School

Florida International University, 2011 


\section{DEDICATION}

I dedicate this dissertation to my son, Dibyanshu - his love and affection is my driving force for completing this research. 


\section{ACKNOWLEDGMENTS}

I sincerely thank my advisor, Dr. WonBong Choi, for his extensive support throughout the span of my $\mathrm{PhD}$ research - without his continued support and encouragement, it would not have been possible for me to finish the dissertation today. He is a key factor in successful completion of this dissertation.

My heartfelt thanks to Dr. Arvind Agarwal, who is also one of my committee members and his $\mathrm{PhD}$ student/post-doc Dr. Debrupa Lahiri for their continued and extensive support. An important part of this dissertation is quantification of CNTsubstrate bonding by a novel nano-scratch technique. It would not have been possible to execute that important study without continuous support, suggestion, encouragement and most active participation in each and every step - from experiment planning, through experimentation, till paper writing - from Dr. Agarwal and Dr. Lahiri. It was a good learning experience for me to work with them.

I sincerely thank Dr. Norman D.H. Munroe and Dr. Joong-ho Moon for serving on my dissertation committee and for their timely advice and support throughout my $\mathrm{PhD}$ research. I am also thankful to Dr. Surendra Saxena - he was always very supportive with his advices and provided me immense help with the research facilities.

My special thanks to all my present and past group mates - Dr. Raghunandan Seelaboyina, Dr. Jun Huang, Dr. Harindra Vedala, Dr. Ved Prakash Verma, Santanu, Chiwon and Divya. My special thanks to Dr. Raghunandan Seelaboyina and Dr. Jun Huang, who were very much supportive during my initial days, when I was learning about this new field of research. I also thank all other friends and colleagues in FIU. I must mention about Lyci, Puneet and Shadab - they have extended their hands during my 
research, whenever I needed any help from them. Dr. Kantesh Balani and Dr. Anup Kumar Keshri deserve special mention as my close friends, who were extremely supportive to my technical and personal issues.

I am also indebted to Dr. Rajarshi Banerjee (University of North Texas, Denton, TX, USA) and his post-doctoral researcher Dr. Jun Y. Hwang for their continued support in performing HRTEM and XPS studies. They were very much enthusiastic and used to manage time quickly for my samples, in spite of their own busy schedule.

I sincerely acknowledge support from Dr. Yang-Kook Sun (Hanyang University, Seoul, Korea) and his PhD students, for continuous support regarding the Li-ion battery studies. It was a very fruitful collaboration with them that has helped a lot in fulfillment of the aim of my dissertation. I personally enjoyed technical discussions with them and learnt firsthand about battery studies from them, during my visit to Hanyang University in May-June, 2009. I also thank Dr. Hyeongtag Jeon, Hanyang University, Seoul, Korea, for an important collaboration regarding atomic layer deposition.

My heartfelt thanks to Mr. Neal Ricks and Dr. Liu- they were very much supportive to my experiments throughout my research period. Without their extensive help, it could not have been possible for me to finish my studies in a timely manner. I have learnt a lot from both of them. I am also thankful to our department staffs - Maite and Martha - they were always available for reducing my involvement in many administrative and purchase related duties.

I am thankful to University Graduate School, FIU for supporting me through Dissertation Evidence Acquisition (DEA) Fellowship and Dissertation Year Fellowship (DYF). I also acknowledge financial support from AFOSR grant (FA9550-09-1-0544), 
NSF grant (NSF-CMMI-0900583) and WCU program through Korea Science and Engineering Foundation funded by the Ministry of Education, Science and Technology (R31-2008-000-10092) for partly supporting research work during different time periods.

On the personal front, I am grateful to my parent-in-law - I would not have decided to join FIU for PhD without their strong encouragement and continuous support. Apart from pouring in mental support, they have also taken the responsibility of bringing up my son, so that both me and my wife, Debrupa can concentrate fully on our studies and research. 'Thanks' is too small a word to express my feelings to them. I want to thank my parents, too and all those relatives, who were always supportive to us.

I am not sure how to express my feelings for my son - Dibyanshu. I missed him throughout these 4 years - it was extremely painful to stay away from him. I hope to stay with him very soon and give him lot of extra love that he missed in last four years. His love and affection are my driving energy - this dissertation is a small gift to him.

Lastly, I can not miss the most important person in my life - my wife and best friend, Debrupa. We always stayed hand-in-hand through all ups and downs of life in last 14 years; we could not even miss doing our $\mathrm{PhDs}$ together! Our academic backgrounds being similar, we could share our thoughts and work together during $\mathrm{PhD}$, especially during the nano-scratch work. She was the only person who was always with me during my Miami days - supporting with personal touches and technical feedbacks. I do not know any word to express my feelings for her - let it be left to the readers' understanding!! 


\title{
ABSTRACT OF THE DISSERTATION
}

\section{CARBON NANOTUBE BASED SYSTEMS FOR HIGH ENERGY EFFICIENT}

\section{APPLICATIONS}

\author{
by \\ Indranil Lahiri \\ Florida International University, 2011 \\ Miami, Florida

\section{Professor WonBong Choi, Major Professor}

In the current age of fast-depleting conventional energy sources, top priority is given to exploring non-conventional energy sources, designing highly efficient energy storage systems and converting existing machines/instruments/devices into energyefficient ones. 'Energy efficiency' is one of the important challenges for today's scientific and research community, worldwide.

In line with this demand, the current research was focused on developing two highly energy-efficient devices - field emitters and Li-ion batteries, using beneficial properties of carbon nanotubes (CNT). Interface-engineered, directly grown CNTs were used as cathode in field emitters, while similar structure was applied as anode in Li-ion batteries. Interface engineering was found to offer minimum resistance to electron flow and strong bonding with the substrate. Both field emitters and Li-ion battery anodes were benefitted from these advantages, demonstrating high energy efficiency. Field emitter, developed during this research, could be characterized by low turn-on field, high emission current, very high field enhancement factor and extremely good stability during long-run. Further, application of 3-dimensional design to these field emitters resulted in 
achieving one of the highest emission current densities reported so far. The 3-D field emitter registered 27 times increase in current density, as compared to their 2-D counterparts. These achievements were further followed by adding new functionalities, transparency and flexibility, to field emitters, keeping in view of current demand for flexible displays. A CNT-graphene hybrid structure showed appreciable emission, along with very good transparency and flexibility.

Li-ion battery anodes, prepared using the interface-engineered CNTs, have offered $140 \%$ increment in capacity, as compared to conventional graphite anodes. Further, it has shown very good rate capability and an exceptional 'zero capacity degradation' during long cycle operation. Enhanced safety and charge transfer mechanism of this novel anode structure could be explained from structural characterization. In an attempt to progress further, CNTs were coated with ultrathin alumina by atomic layer deposition technique. These alumina-coated CNT anodes offered much higher capacity and an exceptional rate capability, with very low capacity degradation in higher current densities. These highly energy efficient CNT based anodes are expected to enhance capacities of future Li-ion batteries. 


\section{TABLE OF CONTENTS}

CHAPTER

PAGE

1. INTRODUCTION 1

1.1 Field Emission - Application of Carbon Nanotubes 2

1.1.1 Carbon Nanotube Based Field Emitters - Advantages,

Limitations and Scope of Improvement 2

1.2 Lithium Ion Batteries - Application of Carbon Nanotubes 4

1.2.1 Carbon Nanotube as Anode in Li-ion Batteries - Pros and Cons and Scope of Improvement

1.3 Objectives of Present Research

References

2. LITERATURE REVIEW 10

2.1 Field Emission Devices - History and Applications 10

2.1.1 Theory of Field Emission $\quad 12$

2.1.2 Materials Used in Field Emitters $\quad 17$

2.1.3 Carbon Nanotubes in Field Emitters $\quad 19$

2.1.4 Properties Affecting Emission Performance 26

2.1.5 Scope of Improvement in Carbon Nanotube Based Field Emitters 28

2.2 Li-ion Batteries - History and Applications 29

2.2.1 Working Principle of Li-ion Batteries 31

2.2.2 Materials Used as Anodes in Li-ion Batteries 34

2.2.3 Carbon Nanostructures as Anode in Li-ion batteries 37

$\begin{array}{ll}2.2 .3 .1 \text { Fullerne } & 37\end{array}$

2.2.3.2 Carbon Nanotube/ Nanofiber and Graphene 37

2.2.3.3 Specialty Carbon Nanostructures $\quad 40$

2.2.3.3.1 Ordered mesoporous and hierarchically porous carbon 40

2.2.3.3.2 Superfine expanded graphite fiber 42

2.2.4 Scope of Improvement in Li-ion Batteries 42

References $\quad 44$

3. MATERIALS AND METHODS 56

3.1 Carbon Nanotube Synthesis 56

3.2 Preparation of Electrodes and test Devices $\quad 60$

3.2.1 Preparation of Conventional 2-Dimensional Field Emitters $\quad 60$

3.2.2 Preparation of 3-Dimensional Field Emitters 60

3.2.3 Preparation of Transparent Flexible Cathode for Field Emitters $\quad 61$

3.2.3.1 Graphene Synthesis $\quad 62$

3.2.3.2 Transferring Graphene on PET 62

3.2.3.3 Cathode Fabrication $\quad 63$

3.2.3.4 Anode Fabrication 63

3.2.4 Preparation of Li-ion Battery Test Cells 64 
3.3 Structural Characterization 64

3.3.1 Scanning Electron Microscopy 65

3.3.2 Transmission Electron Microscopy 65

3.3.3 Stereological Analysis 66

3.3.4 X-Ray Diffraction $\quad 66$

3.3.5 Raman Spectroscopy 66

3.4 Field Emission Characterization $\quad 67$

3.5 Evaluation of Battery Performance 68

3.6 Other Characterization Techniques 68

$\begin{array}{ll}\text { 3.6.1 Differential Scanning Calorimetry } & 69\end{array}$

3.6.2 Electrochemical Impedance Spectroscopy 69

3.6.3 X-ray Photoelectron Spectroscopy 70

$\begin{array}{ll}\text { 3.6.4 Nano-Scratch } & 70\end{array}$

$\begin{array}{ll}\text { References } & 72\end{array}$

4. RESULTS AND DISCUSSION 73

4.1 Structure and Field Emission Performance of Conventional
2-Dimensional Field Emitters

4.1.1 Structures of MWCNT Based Field Emitters 78

4.1.2 Effect of Substrate on Field Emission Response 83

4.1.3 Effect of Interfacial Layer on Field Emission Behavior 87

4.1.4 Effect of Catalyst Layer on Field Emission $\quad 92$

4.1.5 Other Possible Factors Affecting Field Emission 94

4.1.6 Importance of Interface Engineering on Field Emission 98

$\begin{array}{lr}4.2 \text { Substrate-CNT Bonding Strength } & 100\end{array}$

4.2.1 Bonding Energy Quantification by Nano-Scratch 104

4.2.2 Bonding Energy for MWCNT Structure 105

4.2.3 Bonding Energy for Carbon Nanocone Structure 115

4.3 Structure and Field Emission Performance of 3-Dimensional Field Emitter

119

4.3.1 Effect of Number of Micro-Channels on Field Emission Performance

121

4.3.2 Predicted Current Density 127

4.4 Structure and Field Emission Performance of Transparent Flexible Field Emission Device

129

4.5 Structure and Performance of Carbon Nanotube Based Anode in Li-ion Battery

135

4.6 Structure and Performance of Ultra-thin Alumina Coated Carbon Nanotube Based Anode in Li-ion Battery

4.7 Operational Mechanism of Carbon Nanotube Based Anode in Li-ion Battery

4.7.1 Differential Scanning Calorimetry Studies on MWCNT Anode

4.7.2 Electrochemical Impedance Spectroscopy Studies on MWCNT Anode

4.7.3 X-ray Photoelectron Spectroscopy Studies on MWCNT Anode 
References

5. CONCLUSIONS

6. RECOMMENDATIONS FOR FUTURE RESEARCH

VITA 


\section{LIST OF TABLES}

TABLE

PAGE

Table 2.1: $\quad$ Summary of materials used in field emitters

Table 2.2: Summary of materials (except carbon-based materials) used as 36 anode in Li-ion batteries

Table 2.3: Summary of applying CNT/CNF/graphene as anode in Li-ion batteries

Table 4.1: Nomenclature of the samples used in this study

Table 4.2: Raman characteristics of MWCNT emitters grown on different samples

Table 4.3: Overview of literature reported CNT-substrate adhesion testing methods

102

Table 4.4: Estimated current density from different number of channels (from 128 $1 \mathrm{~cm} 2$ foot-print area) 


\section{LIST OF FIGURES}

FIGURE

PAGE

Figure 1.1: $\quad$ Scheme of the present research plan 8

Figure 2.1: $\quad$ Field and thermionic emission processes in a field-temperature 11 space, for an assumed work function of $4.5 \mathrm{eV}$

Figure 2.2: $\quad$ Surface potential barrier seen by an electron during field electron emission (solid line). Contributions from image potential and applied field are shown by broken and broken-solid line, respectively

Figure 2.3: $\quad$ Research trends for a variety of field emitter materials (decade wise publication list in English-language journals only). Source: scopus.com

Figure 2.4: Field emission response from a vertically aligned CNT array. (a) Current-voltage behavior, along with F-N plot in the inset, (b) stability of emission

Figure 2.5: (a) Schematic design of the carbon nanotube based flat panel display and (b) the actual field emission device showing three different color modes

Figure 2.6: Schematic representation of the device design having a bottomgated structure, using (a) whole insulating layer and (b) a patterned insulating layer

Figure 2.7: Thin MWCNT based field emitter structure using micro-channel plate (MCP). (a) Schematic of the device structure, (b) field emission responses with and without $\mathrm{MCP}$

Figure 2.8: Multistage CNT array field emitter. (a) and (b) SEM images of the structure, (c) field emission response

Figure 2.9: Publication status (in English-language journals only) showing high interest in Li-ion battery research (source: scopus.com)

Figure 2.10: Schematic drawing of the structure of Li-ion batteries and mechanism of charge transfer during (a) charging and (b) discharging

Figure 2.11: Publication trend involving carbon nanostructure as anode in Li- 
ion batteries. (Source: scopus.com)

Figure 2.12: TEM image of the ordered mesoporous carbon structure, perpendicular to the direction of hexagonal pore arrangement

Figure 2.13: Hierarchically porous carbon structure - (a) SEM image of the structure and (b) rate capability of the anode

Figure 3.1: A brief schematic presentation of the present research work

Figure 3.2: Schematic process flow-chart for synthesis of MWCNTs on various samples

Figure 3.3: $\quad$ SEM images of the MWCNT structure, grown by CVD

Figure 3.4: A schematic of the processing steps to fabricate the 3D CNTbased field emitter on $\mathrm{Cu}$ substrate

Figure 3.5: A schematic of field emission test set-up (for better visual effect, transparency has been added to the anode of this schematic - in reality, anode is a solid copper sheet)

Figure 3.6: Schematic of the nano-scratch method, followed in the present study. A normal load of $150 \mu \mathrm{N}$ was used for all the scratch tests

Figure 4.1: $\quad$ Structure of CNTs grown on $\mathrm{Cu}$ - and Si-based substrates. (a), (c), (e) SEM images of the MWCNTs on $\mathrm{Cu}-\mathrm{Ti}-\mathrm{Ni}, \mathrm{Cu}-\mathrm{Ti}-\mathrm{Fe}$ and $\mathrm{Cu}-\mathrm{Al}-\mathrm{Fe}$ samples, respectively. (b), (d), (f) SEM images of the MWCNTs on Si-Ti-Ni, Si-Ti-Fe and Si-Al-Fe samples, respectively. All the insets show high magnification images of the respective CNT structures.

Figure 4.2: Raman spectroscopic responses of MWCNTs grown on a variety of samples

Figure 4.3: TEM images of the individual MWCNTs grown on Raman spectroscopic responses of MWCNTs grown on Si-Ti-Ni substrate, showing tips of the CNTs

Figure 4.4: Field emission response of MWCNT emitters on Ti-intermediated substrates. (a) Field emission current, (b) Turn-on fields (shown by the dotted lines), (c) Fowler-Nordheim plots and (d) longtime emission stability of Cu-Ti-Ni-CNT, Al-Ti-Ni-CNT, W-TiNi-CNT, Si-Ti-Ni-CNT and LTCC-Ti-Ni-CNT samples 
Figure 4.5: (a) TEM image of the interface between CNTs and Cu. (b) A schematic of the process showing strong bonding and conductive path formation during CVD growth of CNTs, which leads to enhanced performance of CNTs-on-Cu field emitter. (c) Diffraction spots of $\mathrm{TiC}$ in the selective area diffraction pattern, taken from the interface

Figure 4.6: Field emission responses of MWCNT emitters on Ti-intermediated and $\mathrm{Cu}$-Ti-intermediated substrates. Field emission current and emission stability (insets) of CNT emitters on (a) Al-, (b) W-, (c) Si- and (d) LTCC-substrates

Figure 4.7: Statistical box plot showing CNT diameter distribution of all the samples considered in the present study. Refer to table-1 for nomenclatures of the samples

Figure 4.8: Effect of catalyst (Ni or Fe) on field emission response of CNTs directly grown on $\mathrm{Cu}$ substrates. (a) Field emission current and (b) turn-on field for $\mathrm{Cu}-\mathrm{Ti}-\mathrm{Ni}-\mathrm{CNT}$ and $\mathrm{Cu}-\mathrm{Ti}-\mathrm{Fe}-\mathrm{CNT}$ samples

Figure 4.9: A comparison of the achievements of the present study with the literature reported values, for turn-on field (left half) and field enhancement factors (right half) for CNT based field emitters. While the points denote literature values [13, 21, 49-73], the lines represent the status of the present study

Figure 4.10: Schematic diagram of the working principle of nano-scratch technique

Figure 4.11: Schematic drawing and important geometric ratios of the Berkovich indenter used during nano-scratch study

Figure 4.12: Structural features of CNTs on Cu and Si substrates. (a) Plot of diameter distribution of CNTs on both substrates, at two different growth time, 2 and 30 minutes. Insets show SEM images of catalytic islands on both samples (scale bar length is $200 \mathrm{~nm}$ ). (b) Density of CNTs for both the samples, after 1 and 2 minutes of CVD growth

Figure 4.13: 2-dimensional scanning probe microscopy (SPM) images of the nano-scratches, made on samples with Si-substrate. From left to right, (a) scratches are visible on bare sample, (b) only catalyst deposited sample and (c) sample after CNT growth

Figure 4.14: Nano-scratch through CNTs on different substrates. Lateral force response during nano-scratch tests on (a) $\mathrm{Cu}-\mathrm{CNT}$ and (b) Si- 
CNT samples. Curves represent force required to scratch bare substrates, substrates with only catalysts and substrates after CNT growth. (c and d) SEM images of nano-scratches through the CNT structure on $\mathrm{Cu}$ and $\mathrm{Si}$ substrates, respectively. Insets show higher magnification SEM images of the scratches (scale bar length is $1 \mu \mathrm{m}$ )

Figure 4.15: Scanning probe microscopy (SPM) images of the (a) $\mathrm{Cu}$ and (b) Si substrates after catalyst deposition

Figure 4.16: Plot of lateral force increment for both $\mathrm{Cu}-\mathrm{CNT}$ and Si-CNT samples, after 2 minutes and 30 minutes of growth time

Figure 4.17: Comparison of adhesion energy of individual CNTs on both $\mathrm{Cu}$ and Si substrates

Figure 4.18: SEM images of nano-patterned carbon nanocone structure - (a) before nano-scratch, imaged from top, showing presence of multiple CNCs in each catalytic islands and (b) before nanoscratch, imaged at a tilt angle of $\sim 40^{\circ}$. All scale bars in this figure are $1 \mu \mathrm{m}$

Figure 4.19: Nano-scratch on nano-patterned carbon nanocone structure. (a) SEM image of the CNC structure after nano-scratch, showing removal of $\mathrm{CNCs}$, imaged at a tilt angle of $\sim 40^{\circ}$. (b) Lateral force response during nano-scratch test on Si-CNC sample, comparing effects on bare substrate and through CNCs. The upper inset shows a schematic of the process, uprooted CNCs being shown in a different color. The lower inset shows corresponding SEM image. All scale bars in this figure are $1 \mu \mathrm{m}$

Figure 4.20: Representation of the effect of substrate thickness and channel diameter on total active surface area available for CNT growth (values mentioned against each line in the plot is the channel diameter)

Figure 4.21: Structure of the 3-D field emission device. (a) Micro-channels created on the $\mathrm{Cu}$ substrate. (b) MWCNTs synthesized around and (c) within the micro-channels. (d) Comparison of Raman spectra, before and after field emission tests, showing D and G peaks - signature peaks for CNTs

Figure 4.22: Field emission response of the 3-dimensional cathode structure (numbers mentioned within the plots are the number of microchannels in corresponding samples). (a) Field emission current density from the emitters having different number of channels, 
under DC bias. Inset shows field emission image, captured using green phosphor coated ITO glass as anode. (b) FowlerNordhiem (F-N) plot of the emitters showing straight line behavior. (c) Field emission current density from the emitters having different number of channels, under AC bias. (d) Stability of emission during continuous, long-time operation, under AC bias. (e) Field emission response of emitters under DC bias, after 10 hours of exposure during stability test, showing emitter structure stability. (f) Comparison of current density from 2-D and 3-D CNT based emitters ['red square': data for 2-D CNT based emitter on $\mathrm{Cu}$ substrate (taken from section 4.1); 'green triangles': data from 3-D CNT based emitter on alumina substrate (taken from Seelaboyina et al, ref. 8)]

Figure 4.23: Process flow for cathode and anode preparation. Schematic of assembled full flexible and transparent $\mathrm{FE}$ device showing CNT-graphene/PET cathode and phosphor coated graphene/PET anode

Figure 4.24: Structure of the graphene-CNT hybrid emitter - SEM image of PVA coated CNTs on graphene/PET film

Figure 4.25: (a) Images of flexible and transparent anode and cathode. (b) Fully transparent and flexible FE device assembled on glass tube ("FIU" character can be seen from the front side of the FE device). Inset shows FE image from the device. (c) Emission current versus electric field characteristics of FE device operating under DC bias voltage. Inset shows corresponding F$\mathrm{N}$ plot. (d) Emission current versus electric field characteristics of FE device operating under AC bias voltage. Inset shows corresponding F-N plot

Figure 4.26: Emission stability of the graphene-CNT hybrid field emitter, under DC bias

Figure 4.27: Electrochemical characteristics of the proposed CNT-based electrode structure. (a) First two charge-discharge cycles of the MWNT-on-Cu anode, at $38 \mathrm{mAg}-1$ rate. (b) Reversible capacity of the MWNT-on- $\mathrm{Cu}$ anode, at different C-rates. (c) Exceptional stability of the reversible capacity $(\sim 900 \mathrm{mAhg}-1)$ of the MWNT-on-Cu anode in long-run, at $372 \mathrm{mAg}-1$ rate. (d) Coulombic efficiency of the MWNT-on-Cu anode, showing 138 very high efficiency, except for the first cycle. After initial 5 cycles, the efficiency remained more than $99 \%$ 
Figure 4.28: Structural comparison of the proposed anode structure in different stages. (a) X-ray diffraction patterns and (b) Raman spectroscopic analysis

Figure 4.29: Structure of the electrode in as-grown, lithiated and delithiated states. (a) SEM and (b) TEM images of the as-grown MWNTon- $\mathrm{Cu}$ structure (insets are at higher magnification). (c) TEM images of twisting in the as-grown sample. (d) and (f) show TEM images of the CNTs in lithiated condition (FFT image at inset). (e) and (g) are TEM images of the CNTs in delithiated condition, showing absence of the second phase

Figure 4.30: (a) TEM image of MWCNTs showing fine nanocrytalline particles on the MWCNTs surface, (b) higher magnification of circled region shown in (a), (c) HRTEM image on the one of the nanocrystalline particle showing the lattice fringes, (d) Inverse FFT image indicated by arrow from the filtered FFT showing 4fold symmetry of reciprocal lattice fringes

Figure 4.31: A schematic (not to scale) of the proposed lithiation-delithiation mechanism, showing huge amount of $\mathrm{Li}+$ ion intercalated to walls of CNTs during lithiation and de-intercalation of most of the ions in delithiation

Figure 4.32: Structural analysis of the proposed anode. (a) HRTEM image of substrate-CNT interface, showing well-bonded interface and presence of interfacial phase. (b) Selective area diffraction pattern showing formation of $\mathrm{TiC}$ at the interface

Figure 4.33: A schematic (not to scale) of the proposed anode structure, showing its advantageous features

Figure 4.34: Comparison of reversible specific capacities of literature reported values of different types of anodes and that of the electrode used in present study, over a range of current rates. The interfacecontrolled MWCNT-on-Cu electrode has shown 1.5-5.5 times higher capacities than all reported carbon-based anodes

Figure 4.35: Structure of the anode material. (a) SEM images of the ALD alumina coated MWCNT; the inset shows high magnification image of individual MWCNTs. (b) TEM image of individual CNTs, showing the ultra-thin coatings of alumina on CNTs. (c) Lattice fringes showing alumina $(0.21 \mathrm{~nm})$ and CNT $(0.34 \mathrm{~nm})$. (d) TEM images of few CNTs. The inset shows the high angle angular dark field scanning transmission electron microscope (HAADF-STEM) image from the same location. (e) and (f) 
Spot elemental analysis performed at different positions on the wall of a few CNTs. Both analysis show presence of $\mathrm{Al}$ and $\mathrm{O}$, indicating that alumina has covered the walls of the CNTs forming a thin and discontinuous coating on CNTs. (g) HRTEM image from walls of one of the MWCNTs, showing a presence of multiple walls

Figure 4.36: Lattice fringe image showing CNTs $(0.34 \mathrm{~nm})$ and (400) plane of Li9A14 $(0.45 \mathrm{~nm})$ on the outer surface of CNTs (after lithiation)

Figure 4.37: Electrochemical performance of the anode material. (a) Chargedischarge behavior of the CNT anode with alumina coating in first two cycles. (b) Rate capability of the alumina-coated CNT anode at five different current rates $(114,228,372,558$ and $1116 \mathrm{~mA} \mathrm{g-1,} \mathrm{respectively} \mathrm{For} \mathrm{comparison,} \mathrm{theoretical} \mathrm{specific}$ capacity of graphite is included. (c) Specific capacity retention ability of the electrode in higher number of cycles. Numerical values mentioned in figure $\mathrm{b}$ and $\mathrm{c}$ denotes the current rate $(\mathrm{mA}$ g-1), at which charge-discharge tests are performed. (d) Coulombic efficiency and irreversible capacity loss, as a function of number of cycles

Figure 4.38: A schematic of the advantageous features of ultrathin alumina coated MWCNT anode for Li-ion batteries

Figure 4.39: A comparison plot showing the specific capacities different anodes (as available in open literature) and that of the anodes in the present study, as a function of current density

Figure 4.40: Differential scanning Calorimetry plots MWCNT anode, during different stage of preparation and different states of lithiation/de-lithiation

Figure 4.41: Electrochemical impedance spectroscopy results for MWCNTon- $\mathrm{Cu}$ anodes, at different conditions of charge/discharge. (a) Nyquist plot for the whole frequency domain. (b) Nyquist plot for the frequency range $10 \mathrm{kHz}-100 \mathrm{mHz}$. (c) and (d) are Bode plots showing absolute impedance and phase-shift

Figure 4.42: Equivalent circuit with mixed charge transfer and diffusion control, for MWCNT-on-Cu anodes

Figure 4.43: Li 1s, C 1s, O 1s, F 1s and P 2p XPS spectra of the anode after (a) 1st lithiation, (b) 1st de-lithiation, (c) 2nd lithiation, (d) 2nd de-lithiation and (e) 55th de-lithiation cycles 


\section{INTRODUCTION}

"Energy" is being considered as one of the most important challenges for today's scientific community. As the source of conventional energy is fast depleting, it is imperative to search for alternate sources and save energy in all possible applications. Moreover, sources for conventional energy are the main cause behind increasing pollution and resulting global warming, which are serious threats to our habitat. Possible solutions to these issues could be developing alternate non-polluting energy sources (e.g., using natural resources like solar, wind power etc.), generating energy in a much more efficient way and minimizing energy loss, thus, saving energy, in each and every application. The main aim of this research work is to develop high efficiency energy sources, through two different applications: (a) cold field electron emission sources, which have potential applications in high power microwave devices, display materials and miniature $\mathrm{x}$-ray sources for clinical (imaging) and medical (cancer treatment) purposes and (b) Li-ion (lithium-ion) rechargeable batteries, for powering future portable electronic devices and hybrid cars.

With this goal, the basic idea is to implement interface-engineered carbon nanotubes (CNT) for fabricating these two types of devices, having high energy efficiency. Emphasis will be given on understanding the nature of the interface between CNTs and substrate materials, leading to an optimized interface. An in-depth analysis of electrical and electrochemical properties of the newly developed systems will be conducted to establish their advantages over the existing ones. Structural characterization will support properties of these CNT based systems. 


\subsection{Field Emission - Application of Carbon Nanotubes}

The process of field emission is known to human kind for more than a century, though most of its scientific basis was clarified in 1928 by Fowler and Nordheim $[1,2]$. Tungsten (W) was the first material to be used for field emission [3]. It is also the mostly used field emitter material in commercial instruments. Among other materials, $\mathrm{LaB}_{6}$ has been very popular in practical applications [4]. After discovery of attractive properties of carbon nanotube (CNT) by S. Iijima [5] and demonstration field emission application of CNTs by de Heer et al. [6], CNTs took the center stage of field emission research. Within few years, first CNT-based practical flat panel field emission display was demonstrated [7]. Field emission research involving CNTs took a sharp peak after this period and many other applications have been demonstrated.

\subsubsection{Carbon Nanotube Based Field Emitters - Advantages, Limitations and Scope of Improvement}

In spite of immense popularity of CNTs, which can be related to its fine tips, high aspect ratio, good chemical resistance, efficient thermal and electrical conductivity and exceptional mechanical strength, ample opportunities exist for further development in this field. Two major properties, which need to be enhanced further for CNT-based field emitters, are turn-on field and emission current density. Turn-on field implicates the electrical energy input required to initiate electron emission from the device. In order to achieve an energy-efficient field emitter, turn-on field has to be minimized. On the other hand, emission current density needs to be maximized, in order to gain highest output 
efficiency from the device. Significant developments in these two issues can further expand application of CNT-based field emitters.

For practical device applications, stability of emission and device lifetime are two more extremely important parameters. Emission current achieved from the field emitter, should be stable enough for reproducible performance of the device. Further, its structure should be highly stable, so that device lifetime can be enhanced. For better structural stability, it is necessary to have strong bonding between CNTs and the substrate material used for the device, because failure from this interface has been known to be the main reason for restricting device life [8]. A strong bonding between CNT and substrate material can enhance device life.

With an aim to address all these issues, it seems important to tailor the interface between CNTs and substrate. Moreover, substrate selection appears to play an important role in determining field emission response, as its resistance can significantly influence turn-on field and emission current density. A high-resistance substrate (and interface) will hinder electron movement towards CNTs and negatively affects these two important properties of the emitter. Further, device design enhancements can increase output from the field emitters. Keeping in mind about all these possible solutions to overcome limitations of CNT-based field emitters, this study aims to develop an interfaceengineered CNT growth on low-resistance substrates, which can appreciably enhance field emission response. Design enhancements will also be performed to further advance these achievements and to add new features to CNT based field emitters. 


\subsection{Lithium Ion Batteries - Application of Carbon Nanotubes}

In the present age of extensive use of portable electronic devices, rechargeable (or secondary) batteries play important role as energy storage devices. Efficient use of rechargeable batteries can even lead to hybrid or all-electric vehicles, which is expected to revolutionize the automobile industries. Among all varieties of rechargeable batteries, lithium ion (Li-ion) batteries have quickly become very popular worldwide and are being extensively used in electronic gadgets and devices. Such quick industrial acceptance of Li-ion batteries owes to their excellent properties - high energy density, higher output voltage, absence of memory effect and lower self-discharge rate, as compared to other batteries. Comparing all these advantages, it seems quite obvious that Li-ion batteries are industrially accepted and have attracted lot of research activities in development of lithium ion batteries with higher capacity and better stability in long-cycle operation [9].

In commercial Li-ion batteries, a complex Li-compound is used as cathode and graphite is used as anode. Advancement in nanotechnology and specifically excellent properties offered by CNTs in various applications, propelled replacement of graphite by CNTs with an aim to achieve enhanced properties from the battery [10]. In spite of several such efforts, CNTs have not yet shown attractive properties to be considered as a replacement in commercial Li-ion batteries.

\subsubsection{Carbon Nanotubes as Anode in Li-ion Batteries - Pros and Cons and Scope of Improvement}

CNTs have their obvious advantages over graphite in anodic application in Li-ion batteries. First, CNTs being allotrope of graphite, their chemical interaction with Li is 
expected to be known and should be quite similar to that with graphite. Second, CNTs offer much higher specific surface area (than graphite), which can remarkably enhance amount of $\mathrm{Li}^{+}$ion intercalated into it. Higher $\mathrm{Li}^{+}$ion storage ability allows CNT-based anodes to offer higher capacity. Third, CNTs are much better electronic conductor than graphite, which is anticipated to positively affect charge transfer kinetics [11]. With all these exciting features, CNTs are expected to excel as anode in Li-ion battery application.

In contrary to this expectation, CNTs have not yet offered any such exciting properties, as an anode of Li-ion batteries, such that it can replace graphite in commercial Li-ion batteries. CNT based anodes have offered higher specific capacities than graphite anodes. But CNTs have also shown high irreversible capacity, which is intrinsic to higher surface area of a nano-material and is considered as a matter of serious concern for commercial applications. Further, safety is an important issue in Li-ion batteries and any new material to be used in $\mathrm{Li}$-ion batteries should successfully demonstrate its safety features. With high surface area, which can participate in unwanted exothermic reactions leading to thermal runaway of the battery, CNTs are already presumed to offer less safety for the battery.

With these pros and cons of CNTs for anodic application in Li-ion battery, it is necessary to offer solutions to each of the limiting factors. First of all, specific capacities of CNT-based anodes should be increased to such a level that its application in Li-ion battery offers direct benefit. Secondly, device lifetime, performance reliability and reproducibility should be enhanced for CNT-based anodes. These can be achieved by ensuring structural integrity of the anode during long cycle operations. Further, its safety factors should be demonstrated and if needed, be improved to match with the expected 
levels, such that application of CNT based anodes in commercial Li-ion batteries (offering much higher capacity and faster charging) can be a reality in near future. The present research is focused to target these issues.

\subsection{Objectives of Present Research}

The overall goal of the present research is to demonstrate development of energyefficient devices in two different categories - field emitters and Li-ion batteries, using beneficial properties of carbon nanotubes. This main aim can be achieved through some specific objectives, as delineated below:

Understanding the role of substrate materials on the field emission response of CNTs, grown on them;

Analyzing the function of interface between CNTs and substrate on CNT growth and further on field emission response;

$>$ Optimizing selection of materials as substrate, underlayer, catalyst with the goal of developing an energy-efficient CNT based field emitter;

$>$ Quantifying the bonding energy between CNTs and substrates;

Exploring the possibilities of 3-dimensional design in improving field emission efficiency;

Understanding and analyzing new design and structural modifications in order to impart new functionalities, such as transparency and flexibility, to field emitters;

Dxploring the possibilities of CNTs as anode in Li-ion batteries, with the aims of increasing its capacity, enhancing stability, without affecting safety issues; 
Understanding the charge transfer mechanism in CNT anodes in Li-ion batteries.

Figure 1.1 presents a schematic representation of the planned research works, to be carried out during this study.

The dissertation is segmented into different chapters, sections and subsections to efficiently narrate the developments related to this research work. Chapter 2 will present an overview of the state-of-the-art in the associated issues, specifically highlighting the scope of further research in those fields, which will justify the present research plans. Chapter 3 will give an account of the methodologies implemented in the present research. Fourth chapter, the center point of this dissertation, will present results of the current study and their scientific analysis leading to specific outcomes, in line with the already laid-out objectives. The key findings and achievements will be summarized in chapter 5 . Further scope of research in the related fields and few specific recommendations for nearfuture studies will be proposed in chapter 6 . 


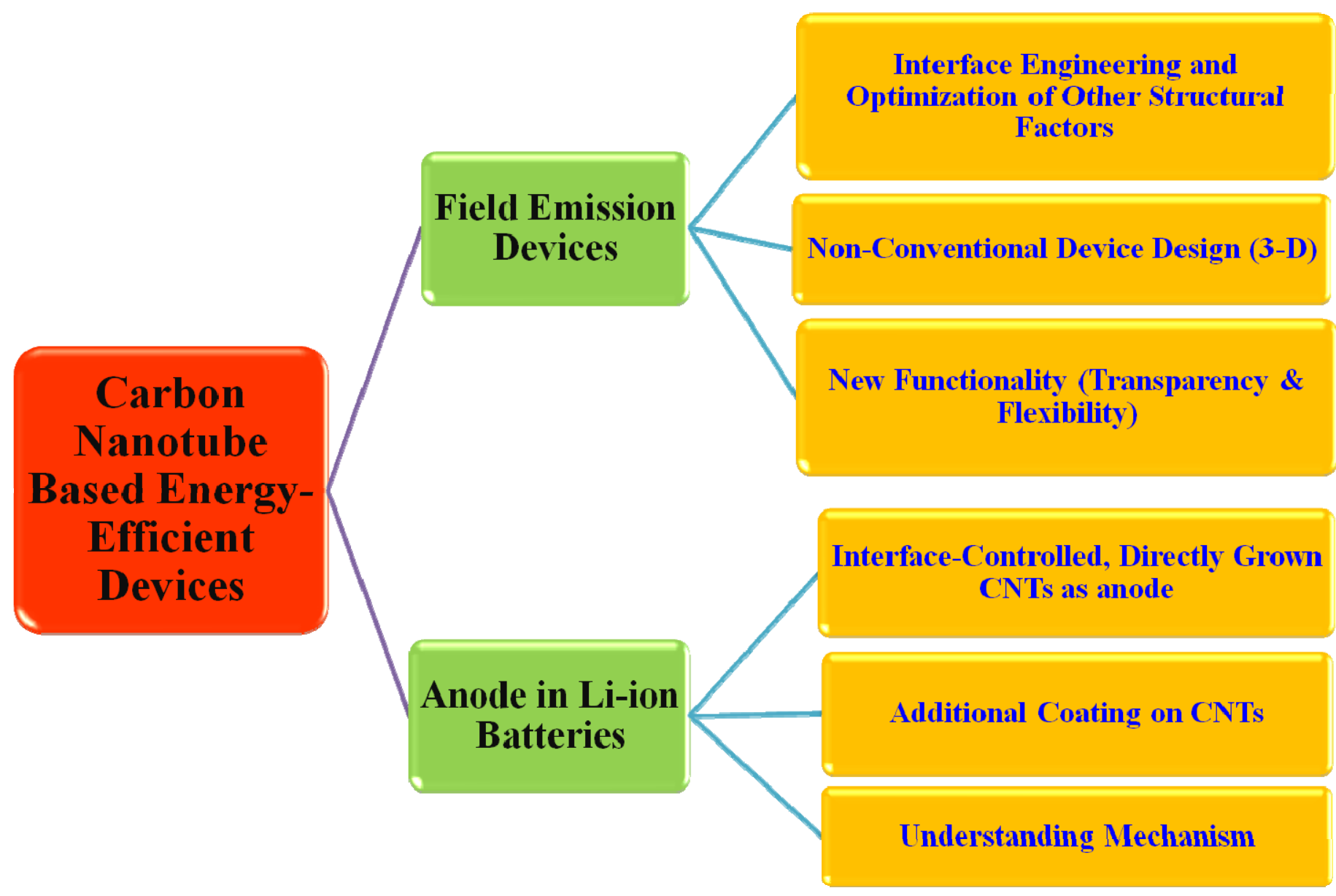

Figure 1.1: Scheme of the present research plan. 


\section{References}

1. R.H. Fowler, L.W. Nordheim. Electron emission in intense electric fields. 1928, Proc. R. Soc. London, Vol. A119, pp. 173-181.

2. L.W. Nordheim. The effect of the image force on the emission and reflection of electrons by metals. 1928, Proc. R. Soc. London, Vol. A121, pp. 626-639.

3. M.J.G. Lee. Field emission of hot electrons from tungsten. 1973, Phys. Rev. Lett., Vol. 30, pp. 1193-1196.

4. K.C. Qi, Z.L. Lin, W.B. Chen, G.C. Cao, J.B. Cheng, X.W. Sun. Formation of extremely high current density LaB6 field emission arrays via e-beam deposition. 2008, Appl. Phys. Lett., Vol. 93, p. 093503.

5. S. Iijima. Helical microtubules of graphitic carbon. 1991, Nature, Vol. 354, pp. 5658.

6. W.A. de Heer, A. Chatelain, D. Ugarte. A Carbon Nanotube Field-Emission Electron Source. 1995, Science, Vol. 270, pp. 1179-1180.

7. W.B. Choi, D.S. Chung, J.H. Kang, H.Y. Kim, Y.W. Jin, I.T. Han, Y.H. Lee, J.E. Jung, N.S. Lee, G.S. Park, J.M. Kim. Fully sealed, high-brightness carbon-nanotube field-emission display. 20, 1999, Appl. Phys. Lett., Vol. 70, pp. 3129-3131.

8. S. Kato, T. Noguchi. Carbon nanotubes and related field emitters: Fundamentals and applications. [ed.] Y. Saito, Wiley-VCH, 2010. pp. 373-376

9. G.A. Nazri, G. Pistoia. Lithium Batteries: Science and Technology. Springer Science+Business Media, New York, 2009.

10. N.A. Kaskhedikar, J. Maier. Lithium Storage in Carbon Nanostructures. 2009, Adv. Mater., Vol. 21, pp. 2664-2680.

11. T. Ishihara, M. Nakasu, M. Yoshio, H. Nishiguchi, Y. Takita. Carbon Nanotube Coating Silicon Doped with Cr as a High Capacity Anode. 2005, J. Power Sources, Vol. 146, pp. 161-165. 


\section{LITERATURE REVIEW}

This chapter is divided into two broad sections. While the first section presents a comprehensive review of scientific investigations performed by other researchers on field emission and application of CNT as a field electron emitter, the following section deals with working principles of Li-ion battery and research efforts to use different materials, specifically carbon nanostructures, as anode of these batteries. An analysis of the presently available knowledge in these fields facilitates to point out the issues of concern, leading to experimental planning of this research work.

\subsection{Field Emission - History and Applications}

Emission, of ions or electrons, from the surface of energized metals, has been known to human kind for more than a century. Emission processes, in a broad sense, can be defined as the process of flow of charge carriers, either ions or electrons, from a highly energized metallic surface to another surface or over some kind of potential barrier. Emission processes can be divided into different categories either depending on the source of energy or on the nature of charge carriers. Based upon the process of energizing the metal surface, emission can be divided as field emission, thermal emission and thermal-field emission. On the other hand, nature of charge carriers leads to either electron emission or ion emission.

The process of field emission was first observed by Robert W. Wood in 1897 [1]. However, it was due to Walter Schottky in 1923 [2] and R.H. Fowler and L.W. Nordheim in $1928[3,4]$ that an insight into the theory of the process was achieved. Putting in simple terms, during this process, an electric field is applied between two electrodes kept 
under vacuum and electrons tunnel through the vacuum, from cathode to anode. This process is generally carried at room temperature or at slightly elevated temperature, allowing high applied electric field. The process could emit ions or electrons.

Thermal emission, more popularly known as thermionic emission, is initiated by supplying thermal energy, so that thermally activated electrons or ions emit out of the surface of the electrode. Though this process was first reported by Daniel Lordan in 1873, it was due to Edison in 1880 that the theory behind the process could be understood. In 1928, O.W. Richardson won the Nobel prize for explaining the thermionic emission process [5-7] and the basic equations were named after him. This process is generally carried out at high temperatures and low or negligible electric field.

In the transition region of these two emission processes, one more process is defined as thermal-field emission process, in which a combination of electric field and thermal energy are used to excite the charge carriers $[8,9]$. This process is, however, not as popular as the other two. Due to complex nature of this process, the governing equations for this process also become complicated, to be used in normal computing facility. Figure 2.1 shows a simple way to distinguish between these emission processes.

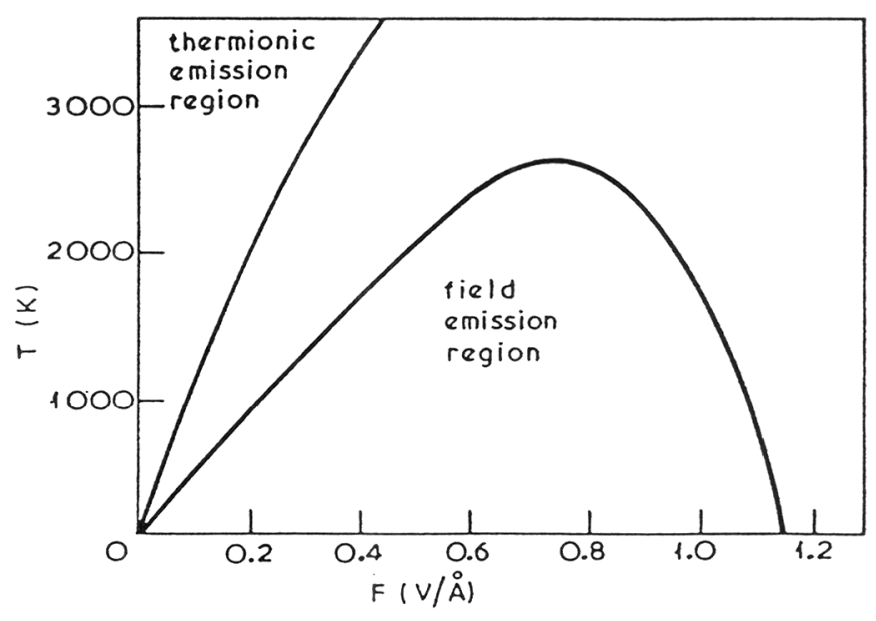

Figure 2.1: Field and thermionic emission processes in a fieldtemperature space, for an assumed work function of $4.5 \mathrm{eV}$ [8]. 


\subsubsection{Theory of Field Emission}

In order to understand the basic process of field electron emission, it is first necessary to estimate the energy required to emit electron from a metal surface. First assumption made in this calculation process is that the metal surface is semi-infinite plate, having its normal in the z-direction. The surface of the metal is taken as $\mathrm{z}=0$. Among all possible energy terms, the most important is Fermi Energy $\left(E_{F}\right)-$ defined as the energy of the highest occupied electron state at absolute zero. Another very important energy term is the Work Function $(\phi)$ of the metal, which is defined as the minimum amount of energy, at absolute zero, that should be supplied to the metal before an electron can escape from its surface. Another energy term, the Image Force (given as $\mathrm{e}^{2} / 4 \mathrm{z}^{2}$, where the negative sign indicates that the attractive force is inward from the metal surface), is defined as the attraction force that an electrons feels towards the plane of a perfect conductor, when situated at a finite distance from it. Adding all these energy terms, the potential energy of an electron on vacuum side of the metal-vacuum interface is given by [8]

$$
\mathrm{V}(\mathrm{z}) \cong \mathrm{E}_{\mathrm{F}}+\phi-\mathrm{e}^{2} / 4 \mathrm{z}^{2}
$$

In the case of a field emission experiment, an external applied field is applied to the surface of the metal. In such cases, the potential energy field seen by an electron is given as

$$
\mathrm{V}(\mathrm{z}) \cong \mathrm{E}_{\mathrm{F}}+\phi-\mathrm{e}^{2} / 4 \mathrm{z}^{2}-\mathrm{eFz}
$$

Figure 2.2, presented in next page, schematically shows the potential energy field of an electron during field electron emission. This shows the amount of energy that needs to be supplied to an electron before it actually could escape from the metal surface. 


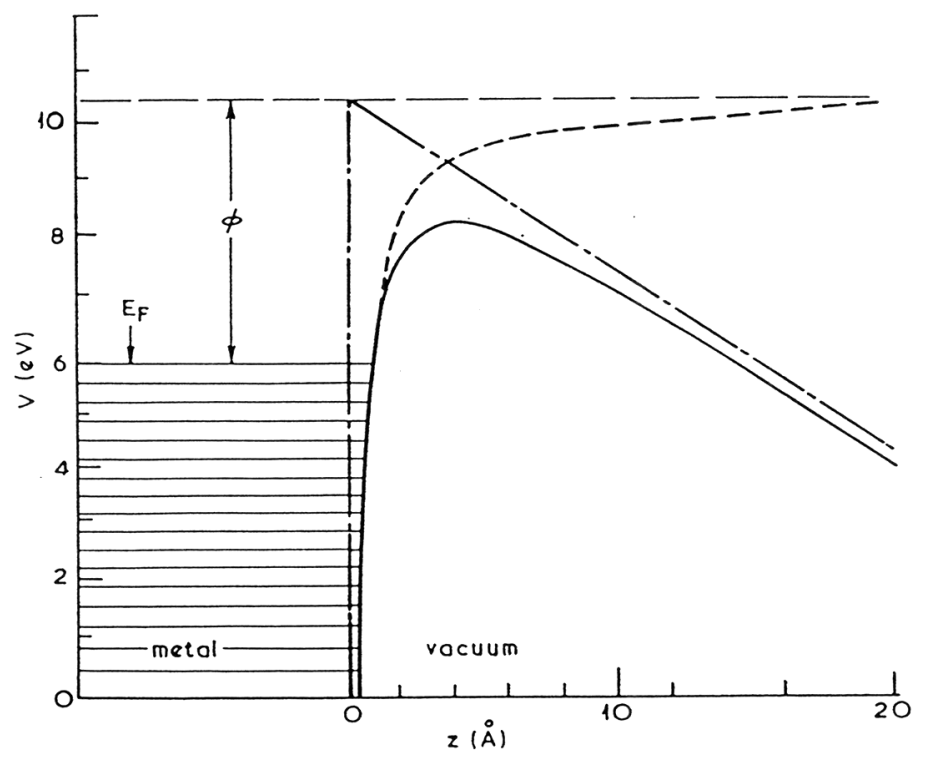

Figure 2.2: Surface potential barrier seen by an electron during field electron emission (solid line). Contributions from image potential and applied field are shown by broken and broken-solid line, respectively. [8]

It may be noted from the figure that the potential barrier takes a triangular kind of shape and it is lowest at highest energy level of electrons. Thus, it is easier for the electrons in higher levels of conduction band to be emitted easily, at a much lower applied field, than the electrons occupying lower energy levels.

In order to calculate the current density obtained in field electron emission, the first thing to calculate is the number of electrons that hit the surface of the metal, from within the metal. The number of electrons, with normal energy between $\mathrm{W}$ and $\mathrm{W}+\mathrm{dW}$, impinging on the surface of the metal, from within the metal is given as

$$
N(W, T) d W=\frac{m d W}{2 \pi^{2} \hbar^{3}} \int_{W}^{\infty} f(E) d E=\frac{m k_{B} T}{2 \pi^{2} \hbar^{3}} \ln \left[1+\exp \left(-\frac{W-E_{F}}{k_{B} T}\right)\right] d W
$$


However, all the electrons impinging the surface of the metal can not emit from the metal surface. It will be decided by the probability $\mathrm{D}(\mathrm{W})$, known as transmission coefficient, to be transmitted through the surface potential barrier. Thus, the field emission current density, number of emitted electrons per unit surface area per unit time multiplied by the magnitude of electronic charge, is presented as

$$
J(F, T)=e \int_{0}^{\infty} N(W, T) D(W) d W
$$

where $\mathrm{T}$ and $\mathrm{F}$ denote temperature and applied field, respectively. Different terms of equation (4) can be written as follows:

$$
\begin{gathered}
N(W, T)=\frac{m k_{B} T}{2 \pi^{2} \hbar^{3}} \ln \left[1+\exp \left(-\frac{W-E_{F}}{k_{B} T}\right)\right] \\
D(W)=\{1+\exp [Q(W)]\}^{-1} \\
Q(W) \equiv-2 i \int_{z 1}^{z 2} \lambda(z) d z \\
\lambda(z)=\left[\frac{2 m}{\hbar^{2}}\left(W-E_{F}-\phi+\frac{e^{2}}{4 z}+e F z\right)\right]^{1 / 2}
\end{gathered}
$$

Using the expressions from equations (5) - (8), equation (4) takes the following shape.

$J(F, T)=\frac{e m k_{B} T}{2 \pi^{2} \hbar^{3}}\left[\int_{0}^{W} \frac{\ln \left\{1+\exp \left[-\left(W-E_{F}\right) / k_{B} T\right]\right\} d W}{1+\exp [Q(W)]}+\int_{W}^{\infty} \ln \left[1+\exp \left\{-\left(W-E_{F}\right) / k_{B} T\right\}\right] d W\right]$ 
Equation (4) is the most generalized form for any kind of emission (field or thermal), from a metal surface. Thus, this generalized form of the equation can be applied to the case of thermionic as well as thermal-field emission also. With proper assumptions, equation (4) leads to formulation of Fowler-Nordheim formula for field emission (at high applied field and low temperature) and to Richardson and Schottky formulae for thermionic emission (high temperature with weak or no applied field). Since, the main focus of this chapter is on field electron emission, it is suitable to mention the FowlerNordheim formula, at this moment.

$$
J(F)=\frac{1.537 \times 10^{10} F^{2}}{\phi t^{2}\left(3.79 F^{0.5} / \phi\right)} \exp \left[-\frac{0.683 \phi^{3 / 2}}{F} v\left(\frac{3.79 F^{0.5}}{\phi}\right)\right] \frac{A}{c^{2}}
$$

Though equation (10), popularly known as Fowler-Nordheim (or, F-N) equation, was originally deduced for metallic electron emitting surfaces of conventional 3dimensional materials, it has successfully been used for other types of (non-metallic and/or non-3-diemnsional) materials, too.

It may be appreciated at this point that in most of the practical cases, the emitting surface is not flat, but curved and small. Thus, removal of an electron from such a surface leads to consideration of a surface energy term. For such a system, the revised form of equation (2) should take the shape of

$$
\mathrm{V}(\mathrm{z}) \cong \mathrm{E}_{\mathrm{F}}+\phi-\mathrm{e}^{2} / 4 \mathrm{z}^{2}-\mathrm{eFz}-2 \gamma^{0} / \mathrm{r}
$$

Analysis of equation (11) immediately shows the reason for better performance of nano-emitters. For a nano-structured material, surface energy $\left(\gamma^{0}\right)$ will be high. If the structure also has small tip radius (r), then the contribution from the term $2 \gamma^{0} / \mathrm{r}$ will be very high, leading to a much smaller value for the potential barrier, V(z). This equation 
shows clearly how an electron could be extracted more easily from a nano-emitter structure, as compared to a micro- or macro-emitter.

In nano field emitters, it has been observed that the macroscopic field $\left(\mathrm{F}_{\mathrm{M}}\right)$, applied to it and the local field (F), at the tip of the emitter is not same and can be related by the following equation.

$$
F=\beta F_{M}
$$

where $\beta$ is known as field enhancement factor.

It is experimentally found that the value of $\beta$ is very high, in the range of 500015000. Several theories exist in support of this behavior; however, none of them could satisfactorily explain this phenomenon properly [10]. One such theory predicts geometrical field enhancement due to nanostructures present on the surface of the emitter. However, atomically flat surfaces were also observed to show efficient field emission and thus, contradict this theory. Another approach [11] predicts negative electron affinity or lower work function supports high local field formation. In contrast to this claim, high work function materials like, hydrogen-terminated diamond was found to emit significantly. In another study, Robertson $[12,13]$ suggested that patch fields, different parts of surface having different work functions, might be responsible for low macroscopic field emission, though experimental observations against this theory also exist. It may be possible that each of these theories contributes towards high field enhancement factor, though any experimental proof of that fact or a detailed theoretical explanation is still not available.

Field enhancement factor has also been known to be directly proportional to the aspect ratio ( $\mathrm{h} / \mathrm{r}, \mathrm{h}$ being height and $\mathrm{r}$ being tip radius) of the emitter. It can immediately 
be concluded that a wire type of structure will show better enhancement as compared to a plate-like structure. It is of no surprise that carbon nanotubes (CNT), having very high aspect ratios, are known as a promising candidate for field emitters.

\subsubsection{Materials Used in Field Emitters}

Field emission is being thoroughly investigated for almost last 50 years. During this time period, different materials have been characterized for their field emission response and some of them have found practical applications, too.

Tungsten (W) was the first material used for field emission. In 1966, Swanson et al. [14] proposed total energy distribution from a tungsten field emitter and the research in this field started immediately. Among other materials, $\mathrm{LaB}_{6}$ has been very popular in practical applications. In last decade, carbon nanotube (CNT) was proposed as an excellent field emission material.

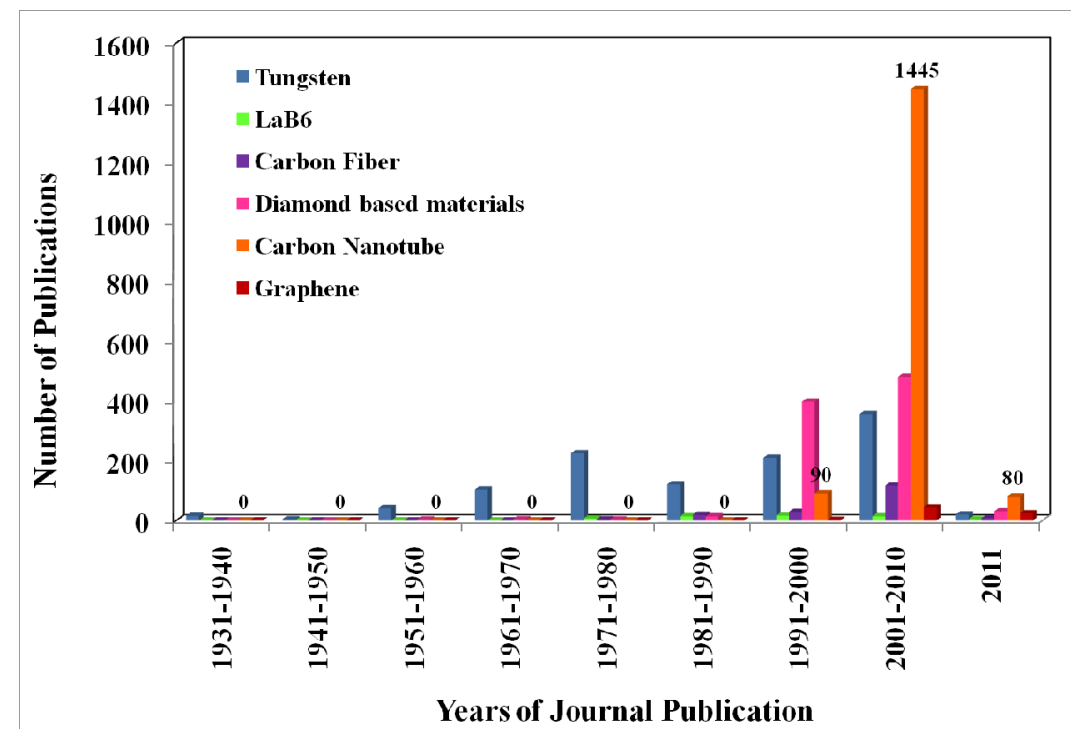

Figure 2.3: Research trends for a variety of field emitter materials (decade wise publication list in English-language journals only). Source: scopus.com. 
Table 2.1 summarizes a variety of materials that has been used for field emission. Due to space limitation, this table uses only a fraction of available literature - actual number of published literature is much higher. Figure 2.3 (previous page) presents a list of publications (decade-wise) for different field emitter materials.

Table 2.1: Summary of materials used in field emitters

\begin{tabular}{|c|c|c|}
\hline Material & Aim of the study & Reference \\
\hline \multirow[t]{5}{*}{ Tungsten } & $\begin{array}{l}\text { Theoretical analysis of total energy distribution of a } \\
\text { tungsten field emitter }\end{array}$ & {$[14]$} \\
\hline & $\begin{array}{l}\text { Emission current and total energy distribution from } \\
\text { different crystallographic planes of tungsten, with and } \\
\text { without various gas adsorption on them }\end{array}$ & [15-19] \\
\hline & $\begin{array}{l}\text { Experimental and theoretical analysis of emission of hot } \\
\text { electrons }\end{array}$ & [20] \\
\hline & Field emission from tungsten nanowire & {$[21]$} \\
\hline & $\begin{array}{l}\text { Multistage tungsten oxide nanowire and its field emission } \\
\text { under poor vacuum condition }\end{array}$ & {$[22]$} \\
\hline \multirow[t]{4}{*}{ Carbon } & $\begin{array}{l}\text { Field emission response from sharpened micro-size } \\
\text { carbon fiber }\end{array}$ & [23] \\
\hline & $\begin{array}{l}\text { Field emission from micro- and nano-sized diamond } \\
\text { emitter arrays }\end{array}$ & [24-27] \\
\hline & $\begin{array}{l}\text { Field emission from single wall- and multi wall- carbon } \\
\text { nanotubes, in the form of arrays or individual nanotube }\end{array}$ & {$[28-42]$} \\
\hline & $\begin{array}{l}\text { Field emission from single-layer, multi-layer and thick } \\
\text { graphene structures }\end{array}$ & [43-49] \\
\hline Silicon & $\begin{array}{l}\text { Large-area arrays of sharply-pointed field emitters on } \mathrm{Si} \\
\text { wafers }\end{array}$ & [50] \\
\hline \multirow[t]{2}{*}{ Molybdenum } & Closely packed arrays of micro-size Mo cones & [51] \\
\hline & Field emission from single crystalline $\mathrm{MoO}_{3}$ nanobelts & [52] \\
\hline
\end{tabular}




\begin{tabular}{|l|l|l|}
\hline $\begin{array}{l}\text { Aluminum } \\
\text { nitride }\end{array}$ & Field emission response of AlN nanotubes & {$[53]$} \\
\hline $\begin{array}{l}\text { Boron- } \\
\text { Carbon- } \\
\text { Nitrogen }\end{array}$ & $\begin{array}{l}\text { Field emission behavior from individual B-C-N nanotube } \\
\text { rope, in situ within a low-energy electron microscope }\end{array}$ & {$[54]$} \\
\hline Copper oxide & $\begin{array}{l}\text { Field emission from aligned cupric oxide nanobelt film, as } \\
\text { a function of temperature }\end{array}$ & {$[55]$} \\
\hline Tin oxide & $\begin{array}{l}\text { Field emission from } ~ 90 \mu m \text { long SnO nanobelt array, } \\
\text { grown on Si wafer }\end{array}$ & {$[56]$} \\
\hline Zinc oxide & $\begin{array}{l}\text { Different morphologies of ZnO nanostructures } \\
\text { nanoneedle array, nanowire, nanopencils, nanorod array } \\
\text { on different substrates and their field emission response }\end{array}$ & {$[57-60]$} \\
\hline $\begin{array}{l}\text { Lanthanum } \\
\text { hexaboride }\end{array}$ & $\begin{array}{l}\text { Micro-tip and nanostructures of LaB } 6 \text { and their field } \\
\text { emission response }\end{array}$ & {$[61-63]$} \\
\hline
\end{tabular}

As can be observed from the figure and the table presented above, carbon nanotubes have attracted most attention as field emitter in the last decade and hence, demands special attention. Moreover, carbon nanotube based field emitter is one of the focus areas of the present research work. This issue will, thus, be discussed in detail in the following sub-section.

\subsubsection{Carbon Nanotubes in Field Emitters}

Research on understanding field emission from CNTs and their application in field emission devices (FED) had taken a big jump in the last decade, owing to its unique structure and beneficial properties. Though field emission from carbon fibers has been known since 1973 [23], it was first in 1995 when a carbon nanotube based field emission electron source has been reported [30]. The device had an array of vertically aligned 
CNTs and the total electron gun was only $0.2 \mathrm{~mm}$ thick. The electron source offered high current density and stability of emission (figure 2.4). With easy scalability to manufacture devices up to hundreds of square centimeters, the authors predicted its suitability for flat panel display applications.
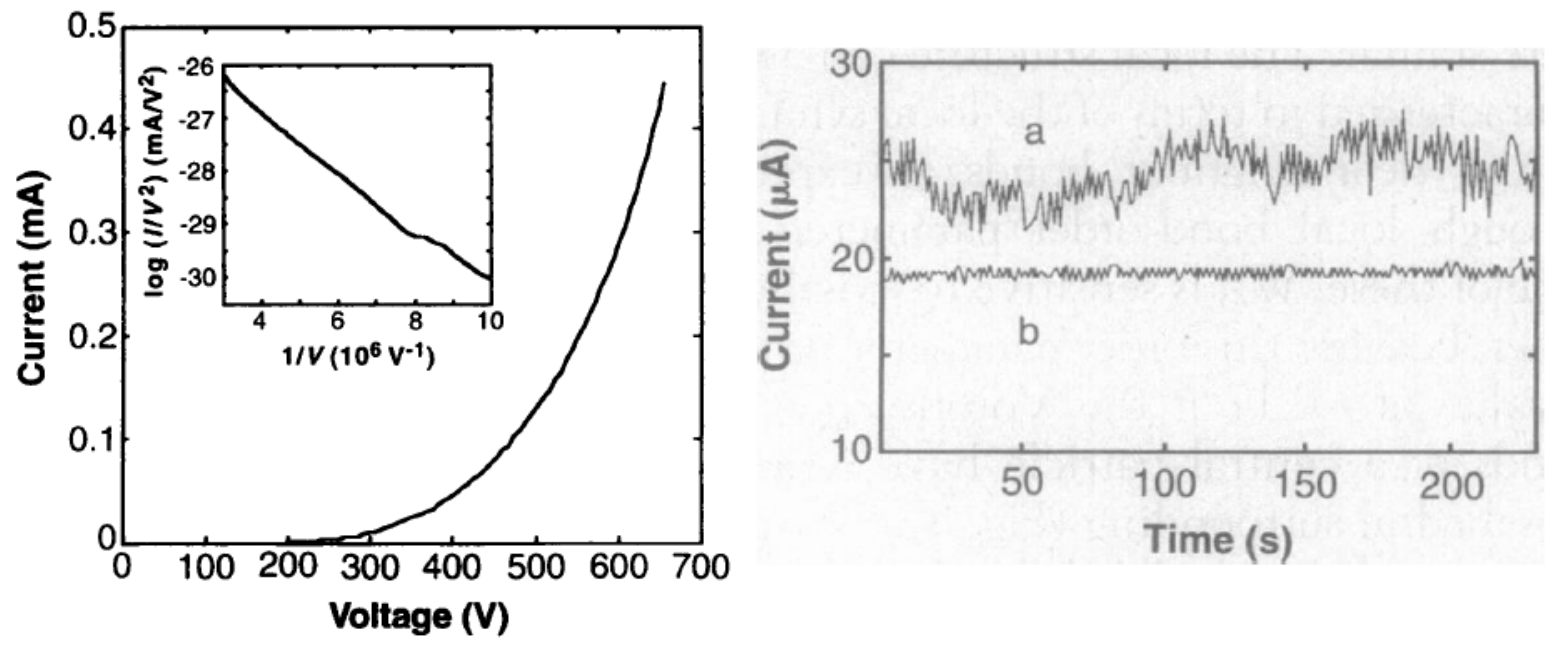

Figure 2.4: Field emission response from a vertically aligned CNT array. (a) Currentvoltage behavior, along with $F-N$ plot in the inset, (b) stability of emission. [30]

Though it took some time to follow this extremely significant study, but in 1999 many exciting results were reported. Two important issues were addressed in two different studies - field emission energy distribution [33], which helped to understand the process of emission in a more detailed manner and environmental stability of field emission mainly in lower vacuum levels or in presence of moisture [34]. Further, Xu et al. [32] reported an inexpensive and controllable process to produce uniform and high density of carbon nanotube emitters on large substrate surfaces. Device, prepared by them, has shown $100-1000 \mathrm{~mA} \mathrm{~cm}^{-2}$ current density at a low macroscopic electric field of 10-15 $\mathrm{V} \mu \mathrm{m}^{-1}$. With support from the knowledge gained from all such studies and 
fulfilling predictions made by de Heer et al. [30], Choi et al. from Samsung Electronics have demonstrated an actual CNT based field emission display (figure 2.5) - 4.5 inches in size [31]. High brightness, good emission current at comparatively low electric field and good stability of emission immediately attracted attention worldwide. Incidentally, a sudden jump in research of CNT based field emission was observed in the next decade, which is continuing till now.
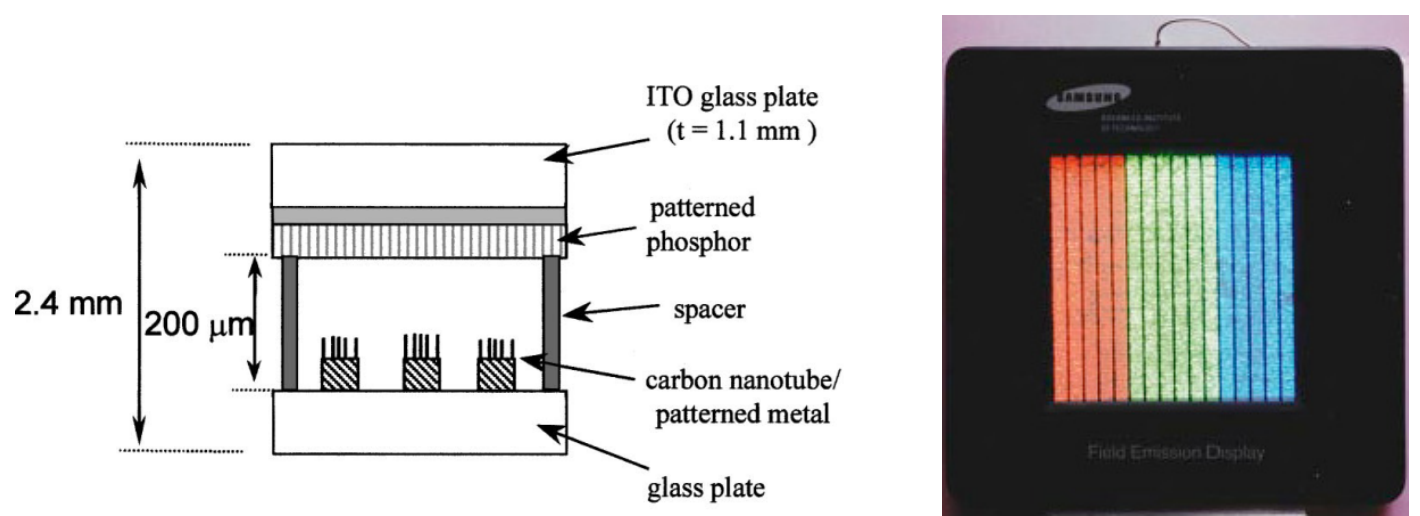

Figure 2.5: (a) Schematic design of the carbon nanotube based flat panel display and (b) the actual field emission device showing three different color modes. [31]

Extending this research further, Saito et al. have successfully manufactured cathode-ray tube type lighting elements and vacuum fluorescence display (VFD) panels, by replacing conventional thermionic cathodes by multiwall carbon nanotubes (MWCNT) [35]. These exciting new device performances were quickly followed by application of new structures - such as CNTs grown on pointed tungsten tips [36], carbon nanofibers [37], single MWCNT [38]. All these initial success stories have sparked wide research activities involving application of CNTs in field emission. As can be seen from figure 2.3, this enthusiasm could be reflected in more than 1400 journal publications (in English language only) during the time period 2001-2010. In the short space of this 
dissertation, it is not possible to refer most of them. However, some of the important findings will be listed below.

As an important development of application of CNTs in field emission displays, it is necessary to understand behavior of CNTs in presence of different types of phosphors. It was observed that emission from CNTs degrade with time in presence of phosphor anode in a field emission back-light unit [64]. Such degradation could be related to degassing of $\mathrm{Zn}$ and $\mathrm{S}$ from the phosphor and modification of tips of CNTs to a nonhexagonal network of carbon atoms, which changed its work function. This study was important to identify a practical device problem.

For wider acceptance of CNTs as a field emitter material, it is important to reduce production cost of CNTs and to make it a environmental friendly synthesis route. In an effort to address this issue, Kumar et al. [65] have shown three dimensional growth of well-aligned high-purity MWCNT arrays on Si, Ni-coated Si and Co-coated Si substrates by thermal decomposition of a botanical carbon source - camphor. When used in field emission device, these MWCNT array have shown $20-30 \mathrm{~mA} \mathrm{~cm}^{-2}$ emission current at an excitation field of $5.6 \mathrm{~V} \mathrm{\mu m}^{-1}$ and stability up to 5 months under continuous operation. This achievement prompted further research activities in this field.

Enhancing field emission current density from the CNT based emitters is always considered as an important step. One simple way could be to add a gate structure to the otherwise diode kind of structure. Such triode type of emitter structure could either be top-gated or bottom-gated. In a bottom-gated type device, in which gate electrodes were located underneath CNT cathodes with an in-between insulating layer, it was observed that emission response was enhanced when a patterned insulator was used [66]. Figure 
2.6 presents the schematic diagrams of device structure. Accumulation of emission electrons in the insulating layer was reduced by patterning the insulator. Simple structure and easy fabrication process of this kind of device is attractive for practical applications.

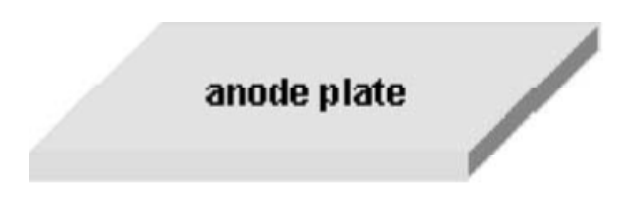

carbon nanot ube

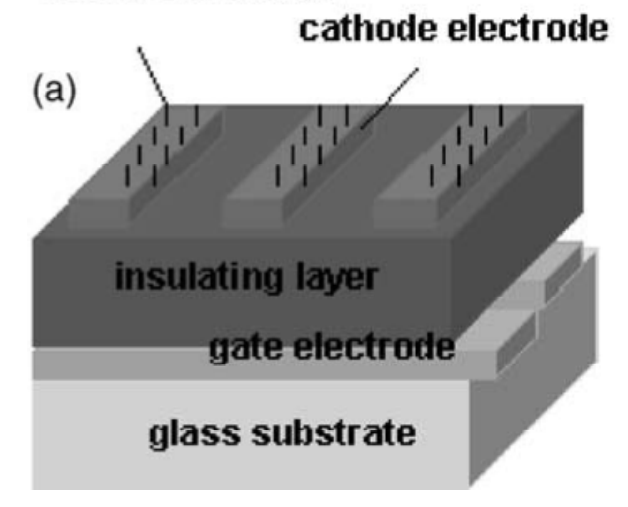

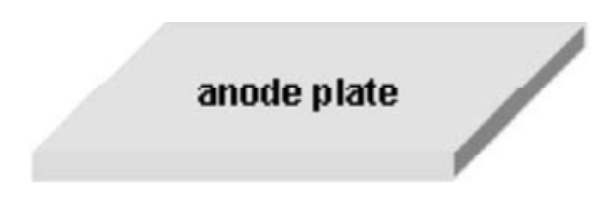

carbon nanotube

(b)

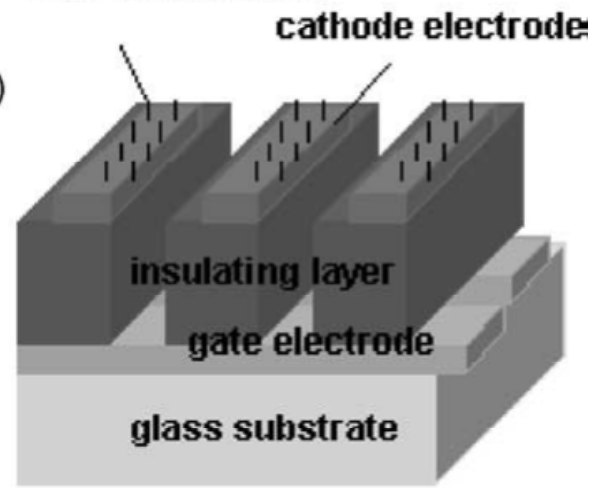

Figure 2.6: Schematic representation of the device design having a bottom-gated structure, using (a) whole insulating layer and (b) a patterned insulating layer. [66]

Constructing a top-gated structure, Seelaboyina et al. [40] have used an electron multiplier micro-channel plate (MCP) as a top-gate-equivalent structure. Figure 2.7 (a) shows the schematic of the emitter structure. Enhancement in field emission current is immediately evident from figure 2.7 (b). Enhancement in the field emission current could be related to dual effect of MCPs. First, MCP operates by avalanche multiplication of secondary electrons, which are generated when incident electrons strike the channel walls of a MCP. A voltage applied across the ends of the MCP creates a field which accelerates 
the secondary electrons along the channel leading to avalanche multiplication. Second, MCP protects the CNTs from irreversible damage during vacuum arcing.

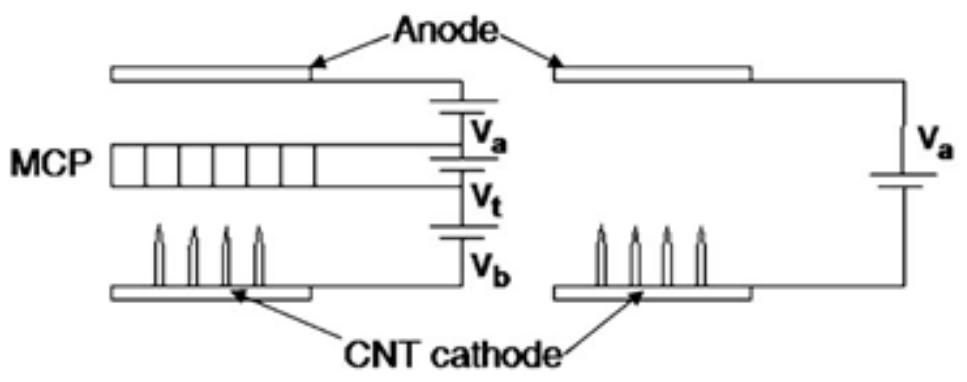

(a)

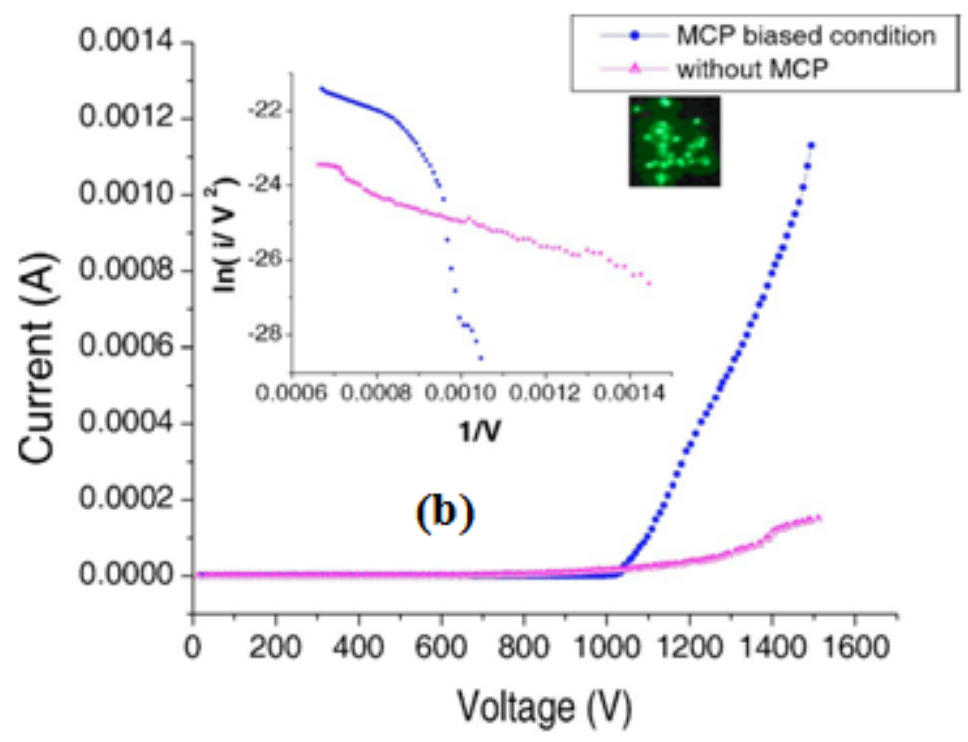

Figure 2.7: Thin MWCNT based field emitter structure using micro-channel plate (MCP). (a) Schematic of the device structure, (b) field emission responses with and without MCP. [40]

Different hybrid structures were also proposed for enhancing field emission current. One example is growth of CNTs on thin W tips, as mentioned before in this subsection. In another variety of hybrid structure, it was shown that a multistage CNT emitter array, consisting of vertical arrays of SWCNTs and thin MWCNTs grown on 
MWCNTs, could significantly enhance emission response [41]. Figure 2.8 presents structure and field emission response of this structure.
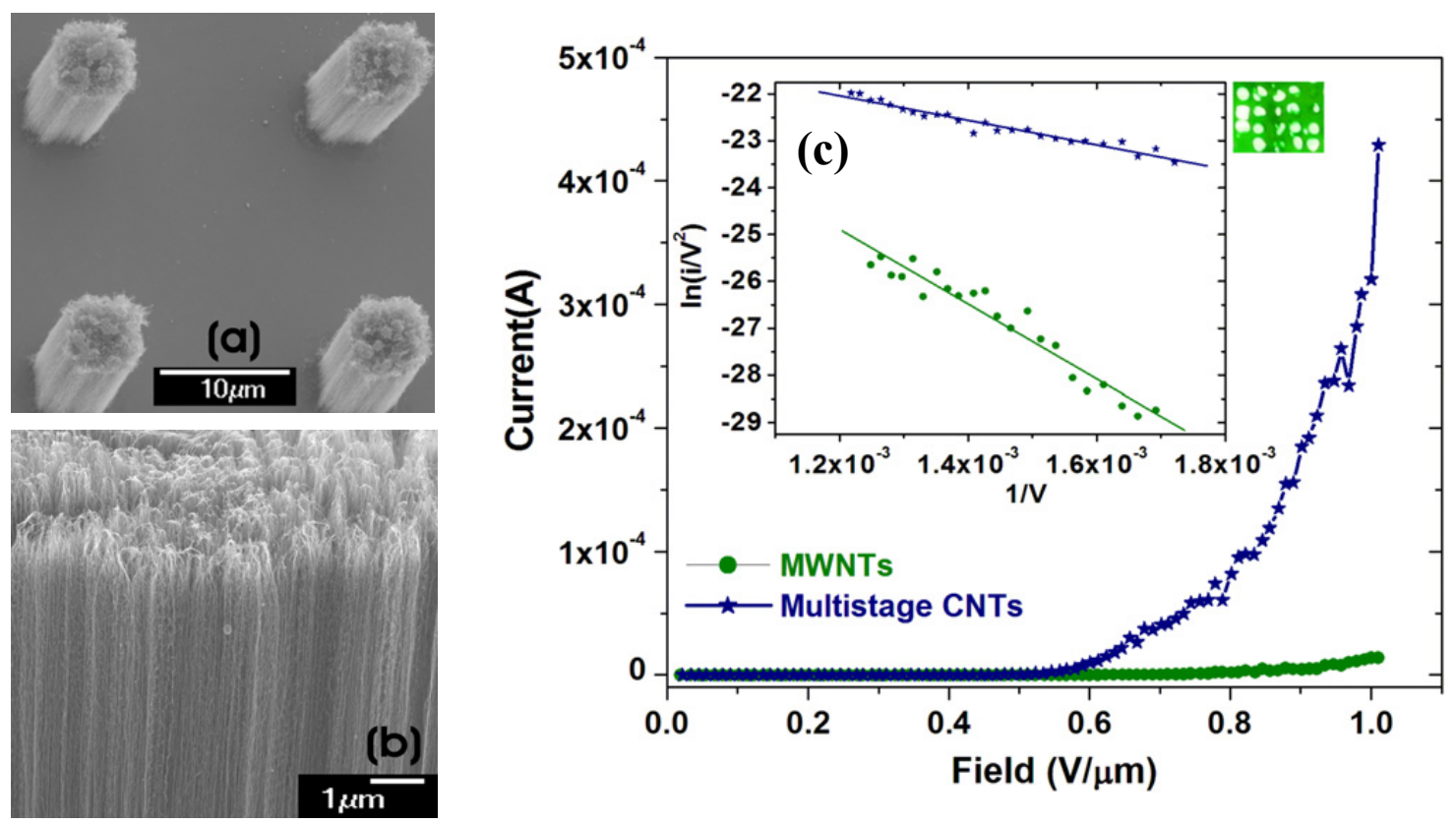

Figure 2.8: Multistage CNT array field emitter. (a) and (b) SEM images of the structure, (c) field emission response. [41]

The beneficial effect of the multistage structure is clearly visible from figure 2.8 (c), which shows more than 10 times enhancement in emission current.

All these interesting research works were well supported parallely by related studies to understand deviation of field emission from conventional F-N relation [67, 68], simulation studies to better understand field emission mechanism $[69,70]$, screening effect [71] and failure mechanism [72] of CNT field emitters and many issues related to growth, morphology, structure, orientation, bonding, presence of metallic nanoparticles etc. Considering space limitation of this chapter, it is not possible to cover all these issues in detail. However, an attempt was made to present important developments and issues 
related to application of CNTs in field emission devices. The following chapter will highlight different aspects that could influence emission behavior of CNT emitters. A good understanding of these issues will direct towards research goals of the present study.

\subsubsection{Properties Affecting Emission Performance}

Before going into discussion about the properties that have significant effect on field emission performance, it is important to know about the process of field emission. Field emission process consists of three steps - electron injection, electron transmission and emission in vacuum [73]. Thus, any property that could affect these process steps might have high impact on controlling the field emission behavior. For the case of CNT field emitters, populating the conduction band of the CNTs, with charge carriers, become important. Thus, it is important to use such a bottom contact, such that it can supply electrons to the CNTs without much resistance. A metallic bottom contact often appears to be beneficial.

Substrate material plays an important role in controlling the field emission response of CNTs. Apart from offering high electrical conductivity (aiding faster electron transport) and thermal conductivity (effectively dissipating heat generated during emission process and thus, minimizing de-bonding of CNTs from substrate), metallic substrates form ohmic contacts with MWCNTs, thus creating a low substrate-CNT barrier. Hence, it is expected that electrons from MWCNTs grown on metallic substrates will be able to tunnel through the energy barrier at a much lower excitation field, thus showing lower $\mathrm{E}_{\text {Tо }}$ values. Among the metallic substrates, the metal with higher work function $(\varphi)$ than CNTs (but, nearer to CNTs) need least energy to inject electrons into 
CNTs, especially metal contact with semiconducting CNTs and hence, show favorable F$\mathrm{N}$ tunneling [74]. On the other hand, metal with lower work function than CNTs forms ohmic contact with semiconducting CNTs. Thus, work function of the contact material is also important. However, it may be recalled here that for practical purposes, MWCNTs are considered as metallic.

Further, structure of CNTs or to be specific, nature of bonds in CNTs is known to influence their field emission response. Under an applied external electric field, the triangular barrier between the Fermi level of the bottom contact and the conduction band of CNTs narrows down, facilitating efficient electron tunneling to the CNTs. While $\mathrm{sp}^{2} \mathrm{C}$ clusters provide sufficient conducting channels, improving electron transfer from the metallic substrate to the emitter tip [75], $\mathrm{sp}^{3}$ portions reduce the electron affinity and thus shorten the potential barrier to aid in an easy escape of electrons (emission) into vacuum [76]. A good combination of $\mathrm{sp}^{2}$ and $\mathrm{sp}^{3}$ bonds in the CNT structure could be the best option to positively influence field emission.

Apart from these issues, type of contact also affects the interfacial resistance for MWCNTs $[74,77]$. For side-contacted CNTs, area of contact directly impact the electron transmission process by imposing a finite energy barrier created by van der waals interaction between metal and CNTs. This interaction was found to be significant for smaller contact areas; though for larger contact areas, better electronic coupling between metal and MWCNTs are reported [74].

Another important factor for a good field emitter is bonding between CNTs with the materials underneath. It has been reported that poor adherence between substrateCNT is known to influence field emission by adding an extra resistance and often leading 
to failure of the device [72]. Good bonding results in lowering of contact resistance and a high, sustained emission current $[78,79]$.

\subsubsection{Scope of Improvement in Carbon Nanotube Based Field Emitters}

Many issues were discussed in the last sub-section, which are known to influence field emission properties of carbon nanotubes. Some of these issues have been studied systematically, but some of them have not been studied thoroughly. Out of those lessstudied but very important issues is substrate material. Some scattered studies have been performed in the past to understand the effect of substrate, underlayer and catalyst material on CNT growth, but no systematic studies have been found to address and clarify this important issue. In 2006, Talapatra et al. [80] have stressed on growth of carbon nanotubes on bulk metallic substrates. Few studies were performed thereafter to study the effect of metallic substrate and catalyst on CNT growth and field emission response [81-86]. However, the effect of materials at the bottom of CNTs on their field emission behavior is still not very much clear. Thus, opportunities exist to clarify this issue and optimize the effects of substrate material on field emission of CNT emitters.

Apart from this important issue, it is also very much necessary to extract more current from the field emission devices. This can be achieved by novel materials and/or design of field emitter devices. Further, CNT based emitters should be constructed in such a way that these could be used efficiently for future flexible displays. Design of such devices will be a step forward in popularizing field emission displays. In the present research work, some of these extremely important issues have been focused. Details 
about the experiments and analysis of results will be presented in chapters 3 and 4, respectively.

\subsection{Lithium Ion Batteries - History and Applications}

Among all varieties of rechargeable batteries, lithium ion (Li-ion) batteries have quickly become very popular worldwide and are being extensively used in electronic gadgets and devices. Using modern Li-ion batteries, efforts are on to produce hybrid or all-electric vehicles, which is expected to revolutionize the automobile industries. Such wide acceptance of Li-ion batteries is facilitated by their intrinsic properties. Li-ion batteries offer high energy density - 160\% higher than Ni-metal hydride (Ni-MH) batteries and $220 \%$ more than Ni-Cd batteries. This means Li-ion batteries of equivalent energy will be much smaller in size and lighter in weight. In other words, keeping battery weight or size similar, Li-ion batteries will produce much higher energy than other rechargeable batteries. Moreover, Li being known as the most electronegative solid material (with standard electrode potential being $-3.04 \mathrm{~V}$; $\mathrm{Li} \leftrightarrow \mathrm{Li}^{+}+\mathrm{e}^{-}$), it is quite expected that $\mathrm{Li}$-ion batteries will offer higher voltages, as compared to other rechargeable batteries. While normal Ni-MH and Ni-Cd batteries offer $1.2 \mathrm{~V}$ and lead acid battery gives $2.1 \mathrm{~V}$, a normal Li-ion battery generates $3.6-3.7 \mathrm{~V}$, depending upon the electrode materials used. Moreover, $\mathrm{Li}$-ion batteries do not have any memory effect and its self-discharge rate $(\sim 5-10 \%$ per month) is lower compared to other batteries $(\sim 30 \%$ per month for common NiMH batteries and $10 \%$ per month for $\mathrm{Ni}-\mathrm{Cd}$ batteries). Comparing all these advantages, it seems quite obvious that Li-ion batteries are industrially accepted and have attracted lot of research activities in development of 
lithium ion batteries with higher capacity and better stability in long-cycle operation [87].

Figure 2.9 below will show a trend in publication of Li-ion battery research activities in English-language journals only.

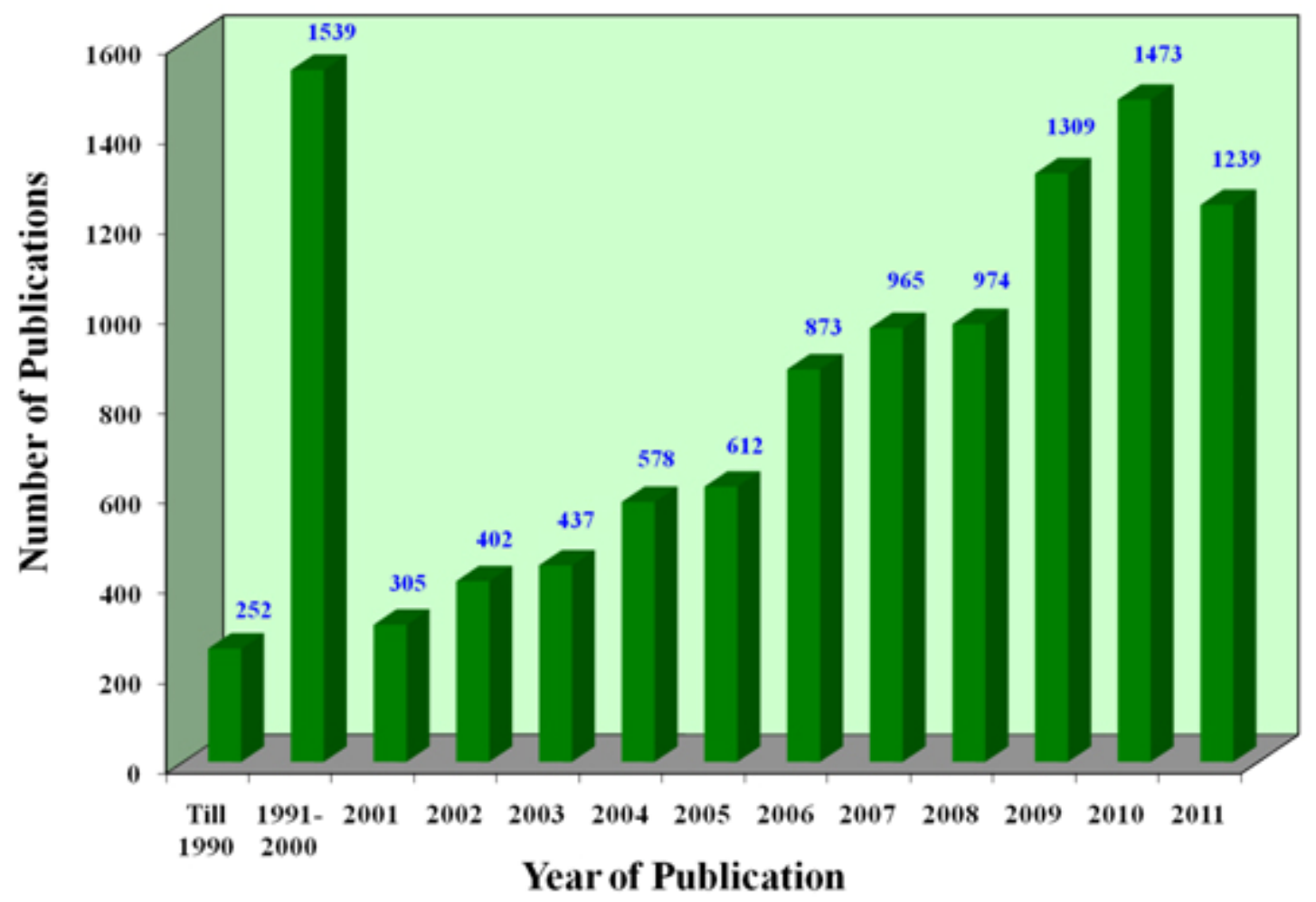

Figure 2.9: Publication status (in English-language journals only) showing high interest in Li-ion battery research (source: scopus.com)

In the present study, stress has been given on development of new CNT-based anode materials. Before explaining the goals of this study, it is necessary to have background knowledge of the system. Following sub-sections are designed to present important issues related to Li-ion batteries, leading towards a plausible justification of the goals of this study. 


\subsubsection{Working Principle of Li-ion Batteries}

The structure of a Li-ion battery, in principle, is same as any other electrochemical cells, having an anode, a cathode, an electrolyte and a separator to avoid direct short-circuiting by not allowing electrons to travel through, but permitting ions to move through it. Figure 2.10 presents schematic drawing of a typical Li-ion battery and shows movement of ions and electrons during charging and discharging cycles.

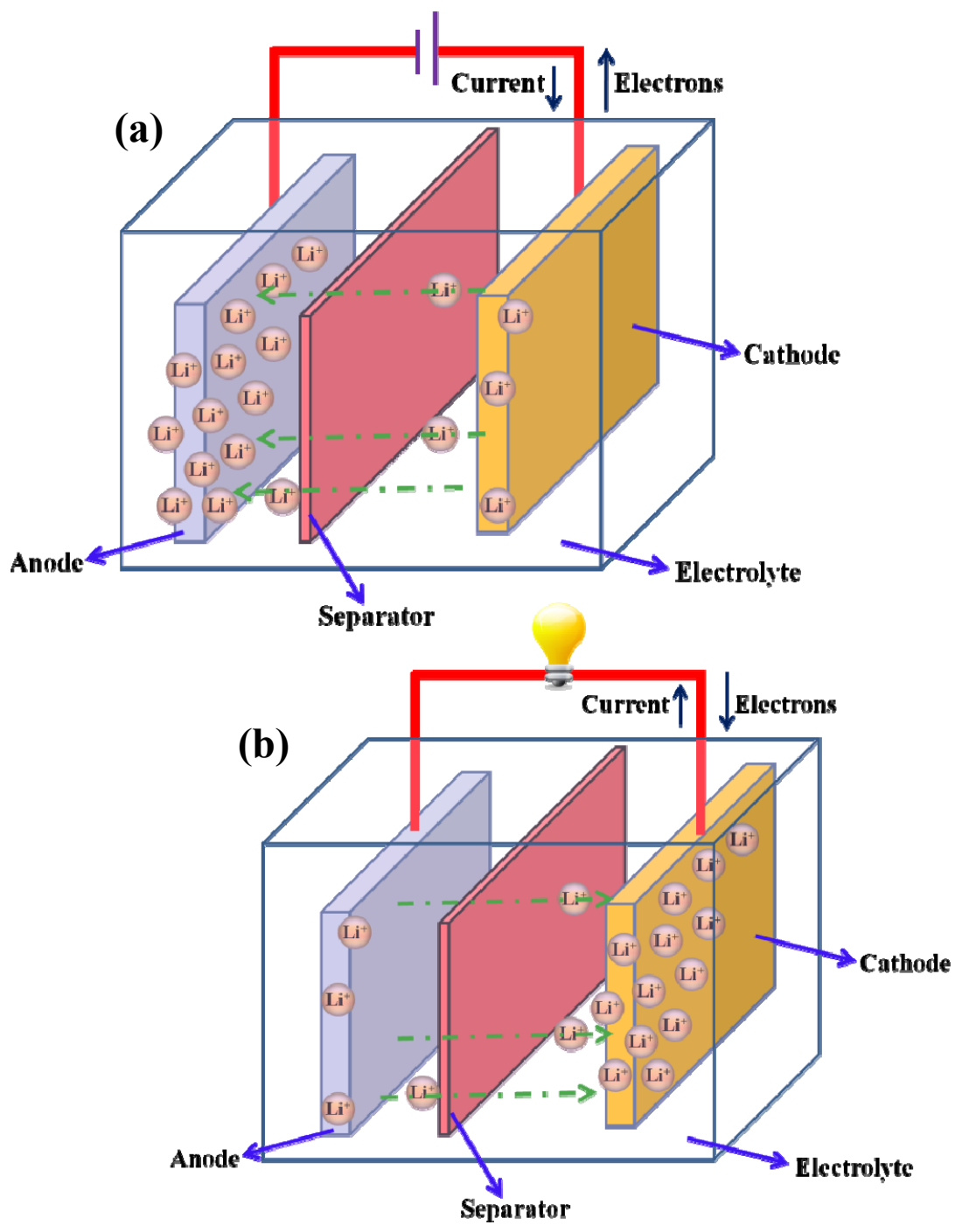

Figure 2.10: Schematic drawing of the structure of Li-ion batteries and mechanism of charge transfer during (a) charging and (b) discharging. 
During charging, external electrical energy aids to break $\mathrm{Li}$ into $\mathrm{Li}^{+}$and electron on the cathode. While $\mathrm{Li}^{+}$ions move through the electrolyte, crossing separator, towards the anode, electrons takes the path of external circuit. On the other hand, during discharging, $\mathrm{Li}^{+}$ions move from anode to cathode and the electrons go through the external circuit (energizing the external 'load') to reach cathode in an effort to recombine with the ions.

$\mathrm{LiCoO}_{2}$ is the most commonly used cathode material, though any compound with the formula $\mathrm{LiMO}_{2}(\mathrm{M}: \mathrm{Mn}, \mathrm{Co}, \mathrm{Ni})$ can be used [88]. Recent advancements have shown good promise for $\mathrm{LiFePO}_{4}$ as the cathode material [88]. Many other complex oxides or mixtures of oxides are used as cathode, but those are still in research scale. Metallic Li is not considered as a cathode material as its compounds offer higher melting temperature than metallic $\mathrm{Li}$, less reactivity with atmosphere and much less tendency to form dendrites during cycling (which can short-circuit the electrodes) [89].

On the other hand, graphite is the most commonly used anode material [90], though other materials like active carbon, mesocarbon microbead (MCMB) etc. are also used often. In research scale, a wide variety of materials are being used and proposed for anode application - next sub-section will present a brief outline of those efforts. Electrolytes used in Li-ion batteries consist of Li-based salt (most popularly $\mathrm{LiPF}_{6}$ ) in an organic solvent, such as ethylene carbonate (EC), diethyl carbonate (DEC) or dimethyl carbonate (DMC) or a combination of these solvents. In most cases, an electrolyte of 1.0M LiPF 6 in EC-DEC (1:1 in volume) is used. Considering the most popularly used components, i.e. $\mathrm{LiCoO}_{2}$ as cathode and graphite (C) as anode, electrode reactions for a Li-ion battery can be written as follows: 


$$
\mathrm{LiCoO}_{2} \leftrightarrow \mathrm{Li}_{1-x} \mathrm{CoO}_{2}+x \mathrm{Li}^{+}+x \mathrm{e}^{-} \text {(at cathode) }
$$

$x \mathrm{Li}^{+}+x \mathrm{e}^{-}+6 \mathrm{C} \leftrightarrow \mathrm{Li}_{x} \mathrm{C}_{6}$ (at anode)

The value of ' $x$ ' depends on the structure of the carbon anode used - for graphite, $x=1$, i.e., the compound formed at the anode is $\mathrm{LiC}_{6}$. This means that for every 6 carbon atoms, one Li atom will be intercalated. For higher lithiation capacity, it is obvious that ' $x$ ' should be high.

From the above-mentioned reactions, it is possible to calculate theoretical specific capacity of the anode $\left(\mathrm{Q}_{\mathrm{A}}\right)$ in $\mathrm{mAh}^{-1}$ using the following equation [89]

$$
\mathrm{Q}_{\mathrm{A}}=(x .96500) /(3.6 \cdot \mathrm{m} \cdot \mathrm{M})
$$

Where ' $\mathrm{M}$ ' is molecular weight of carbon (could be any other element, depending upon anode material used) and ' $\mathrm{m}$ ' is its exponent of $\mathrm{C}$ in the reaction. Inserting appropriate values for a graphite anode $(\mathrm{M}=12, \mathrm{~m}=6$ and $x=1)$, it can be shown that theoretical specific capacity of graphite (or, any other form of carbon, which forms $\mathrm{LiC}_{6}$ with Li) is $372.3 \mathrm{mAh} \mathrm{g}^{-1}$. This is an important information which will be used frequently during the present study.

After knowing about the basics of Li-ion battery, it is important to know about the materials used in a Li-ion battery. Since, the present study is focused at developing a novel anode material for Li-ion batteries, the following discussions will be limited to materials used in anode only. A wide variety of materials have been used as anode in Liion batteries. Section 2.2.2 will present a brief outline of different materials being proposed for anodic application in Li-ion battery, while section 2.2.3 will focus more on the carbon-based anodes only. 


\subsubsection{Materials Used as Anodes in Li-ion Batteries}

Three different categories of materials have been applied (in research scale) as anodes of Li-ion batteries - carbon and carbon nanostructured based materials, oxide based materials led by Sn-oxide and Si-based materials. Few other materials have also been experimented. In this sub-section, a brief outline of all types of structures, except Cbased structures, will be presented.

Though graphite is conventionally used as anode in Li-ion batteries, its low theoretical specific capacity presents ample opportunities for development of new anode materials with higher capacities. $\mathrm{Si}$ and $\mathrm{SnO}_{2}$ are considered to be two important materials for this application, owing to their very high theoretical specific capacities, 4200 and $782 \mathrm{mAhg}^{-1}$, respectively [91, 92]. However, in spite of such high theoretical capacities, neither of these materials could be successfully used as anode in Li-ion batteries as both of these materials suffer from the limitation that during Li-ion intercalation and de-intercalation, they experience huge (300-400\%) expansion and contraction, resulting into pulverization and capacity loss, in high number of cycles. It was shown later that an efficient design of their nanostructures can generate very high capacity, while minimizing the pulverization problem [93-95]. Thus, nanostructures of $\mathrm{Si}$ and $\mathrm{SnO}_{2}$ and their composites with other materials have been studied. Capacity degradation still occurs for some of these anodes in long-cycle operation, though at a much slower rate.

Among other oxide materials, $\mathrm{TiO}_{2}$ and $\mathrm{V}_{2} \mathrm{O}_{5}$ have shown good promise and these two oxides have been used either individually or as a composite with other materials. Though scattered efforts were made to use some other oxides, such as $\mathrm{CuO}, \mathrm{MnO}$ etc., 
but none of them were successful to attract much attention. Many other materials have been proposed for anodic application in Li-ion batteries, e.g. Ge nanowire. Ge is known to have a theoretical specific capacity of $1600 \mathrm{mAh} \mathrm{g}^{-1}$ (forming $\mathrm{Li}_{4.4} \mathrm{Ge}$ compound) [96] and 400 times faster diffusion of Li-ions into it as compared to $\mathrm{Si}$ [97] indicating that $\mathrm{Ge}$ may be an attractive electrode material for high-power-rate anodes. Nanocomposites of Ge with carbon [98] and tin [99] have shown promising capacity and cyclability. However, Ge shows 370\% volume change during lithiation/de-lithiation. In line with the success of Si nanostructures, Ge nanowires have also shown appreciable capacity [96].

Apart from taking care of the volume change (and thus, pulverization issue), introduction of nanomaterials as electrodes in the Li-ion cells, in place of conventional ones, are expected to show higher lithiation capability and an overall better performance simply because of their extremely high surface area as compared to their bulk counterparts. Hence, in recent times, many nanomaterials have been studied for possible application in Li-ion batteries. Virus templated $\mathrm{Au}-\mathrm{Co}_{3} \mathrm{O}_{4}$ nanowire assembly is example of one such novel structure, which has shown good specific capacity [100], though its complicated processing route is restricting its further application. At this point, it must be mentioned that higher surface area of nanomaterials is also responsible for high irreversible capacity loss. Thus, structural and material optimization is extremely important. Table 2.2 presents a summary of a variety of materials used as anode in Li-ion battery, except C-based nanostructures. It may be noted at this point that many other materials have been used for the same purpose; however, in the small space of this chapter, it is not possible to accommodate all such great research efforts. 
Table 2.2: Summary of materials (except carbon-based materials) used as anode in Liion batteries

\begin{tabular}{|c|c|c|}
\hline Material & Achievements of the study & Reference \\
\hline $\begin{array}{l}\mathrm{Si} \\
\text { nanostructures }\end{array}$ & $\begin{array}{l}\text { Si nanowire as anode, showing }>2100 \mathrm{mAh}^{-1} \text { at } 1 \mathrm{C}^{*} \\
\text { rate and } \sim 3500 \mathrm{mAh}^{-1} \text { at } \mathrm{C} / 5 \text { rate; but up to } 39 \% \text { drop } \\
\text { in capacity in } 50 \text { cycles (at } \mathrm{C} / 5 \text { rate) }\end{array}$ & {$[93,101]$} \\
\hline \multirow[t]{9}{*}{$\begin{array}{l}\text { Various } \\
\text { oxides and } \\
\text { their mixtures }\end{array}$} & $\begin{array}{l}\mathrm{ZnO}, \mathrm{SnO}_{2} \text { and their } 1: 1 \text { (molar ratio) mixtures - particle } \\
\text { size in the range of } 200-250 \mathrm{~nm} \text {, reversible capacity is } \\
400,700 \text { and } 450 \mathrm{mAh} \mathrm{g}^{-1} \text {, respectively }\end{array}$ & {$[102]$} \\
\hline & $\begin{array}{l}\text { Tin-based amorphous composite oxide }(\mathrm{TCO})-\text { shows } \\
\text { reversible Li-ion storage capacity }>600 \mathrm{mAh} \mathrm{g}^{-1}\end{array}$ & [103] \\
\hline & $\begin{array}{l}\text { Various nano oxides }-\mathrm{FeO}, \mathrm{NiO}, \mathrm{CoO}, \mathrm{Co}_{3} \mathrm{O}_{4}, \mathrm{Cu}_{2} \mathrm{O}- \\
\text { shows range of capacities from } 1000-600 \mathrm{mAh} \text { g-1 and } \\
\text { some showing } 100 \% \text { capacity retention up to } 100 \text { cycles }\end{array}$ & [104] \\
\hline & $\begin{array}{l}\text { Mixture of metal and oxides }-\mathrm{Sb}-\mathrm{V}_{2} \mathrm{O}_{3} \text { and } \mathrm{Sb}-\mathrm{MnO}- \\
\text { latter shows improved capacity retention ability }\end{array}$ & [105] \\
\hline & $\begin{array}{l}\text { Various sizes of } \mathrm{CoO} \text { and } \mathrm{Co}_{3} \mathrm{O}_{4}(\text { range: } 4-30 \mu \mathrm{m})-\mathrm{CoO} \\
\text { offers best stable capacity } \sim 300 \mathrm{mAh} \mathrm{g}^{-1}\end{array}$ & {$[106]$} \\
\hline & $\begin{array}{l}\text { Porous } \mathrm{Co}_{3} \mathrm{O}_{4} \text { nanotubes - discharge capacity of } 1200 \\
\mathrm{mAh} \mathrm{g}^{-1} \text { at a current rate of } 50 \mathrm{~mA} \mathrm{~g}^{-1}\end{array}$ & {$[107]$} \\
\hline & $\begin{array}{l}\mathrm{MoO}_{x}(x=2 \text { or } 3) \text { nano-particles prepared by hot wire } \\
\text { chemical vapor deposition - capacity of } 620 \mathrm{mAh} \mathrm{g}^{-1} \text { and } \\
93 \% \text { capacity retention in } 50 \text { cycles }\end{array}$ & {$[108,109]$} \\
\hline & $\begin{array}{l}\mathrm{V}_{2} \mathrm{O}_{5} \text { nanoribbon - extremely high } \mathrm{Li} \text { diffusion rate, } \\
\text { which can provide super-high power rate of } 360 \mathrm{C}(10 \mathrm{~s})\end{array}$ & {$[110]$} \\
\hline & $\begin{array}{l}\text { Virus-enabled synthesis of } \mathrm{Au}_{-\mathrm{Co}_{3} \mathrm{O}_{4}} \text { nanowire - } \\
\text { approximately } 1100 \mathrm{mAh} \mathrm{g} \mathrm{g}^{-1} \text { capacity at a current rate of } \\
\mathrm{C} / 26.5\end{array}$ & {$[100]$} \\
\hline Ge nanowire & $\begin{array}{l}\text { High specific capacity }-1141 \mathrm{mAh} \mathrm{g}^{-1} \text { at } \mathrm{C} / 20 \text { rate and } ~ \\
600 \mathrm{mAh} \mathrm{g}^{-1} \text { at } 2 \mathrm{C} \text { rate }\end{array}$ & [96] \\
\hline
\end{tabular}




\subsubsection{Carbon Nanostructures as Anode in Li-ion Batteries}

Advantages of nanostructures are obvious for their application as electrode in Liion batteries. In line with application of nanostructures of $\mathrm{Si}, \mathrm{SnO}_{2}$, Ge etc., a variety of carbon nanostructures have also been attempted for anodic application in Li-ion batteries [111]. Enormous amount of literature exist dealing with carbon nanostructure based anodes - the following sub-sections will present a highlight of these structures.

\subsubsection{Fullerene}

Both $\mathrm{C}_{60}$ and $\mathrm{C}_{70}$ fullerenes were studied for understanding their $\mathrm{Li}$ storage capacities. It was observed that a maximum of $1 \mathrm{Li}$ per $5 \mathrm{C}$ can be accommodated in $\mathrm{C}_{60}$ molecule [112], which presents better situation as compared to graphite $\left(\mathrm{LiC}_{6}\right)$. Hydrogenated fullerenes were found to offer higher Li-storage capacity and better stability [113]. The capacity was also observed to be dependent on the degree of hydrogenation. However, in spite of some good feedbacks, fullerene based anodes were not studied much, because of huge advantages expected from two other carbon allotropes - carbon nanotube and graphene.

\subsubsection{Carbon Nanotube/ Nanofiber and Graphene}

Among the three carbon allotropes - 0-dimensional fullerene, 1-dimensional carbon nanotube and 2-diemnsional graphene, carbon nanotubes have attracted most attention in recent years for application as anode in Li-ion batteries, though application of graphene has also gained quick popularity during last couple of years (figure 2.11). 


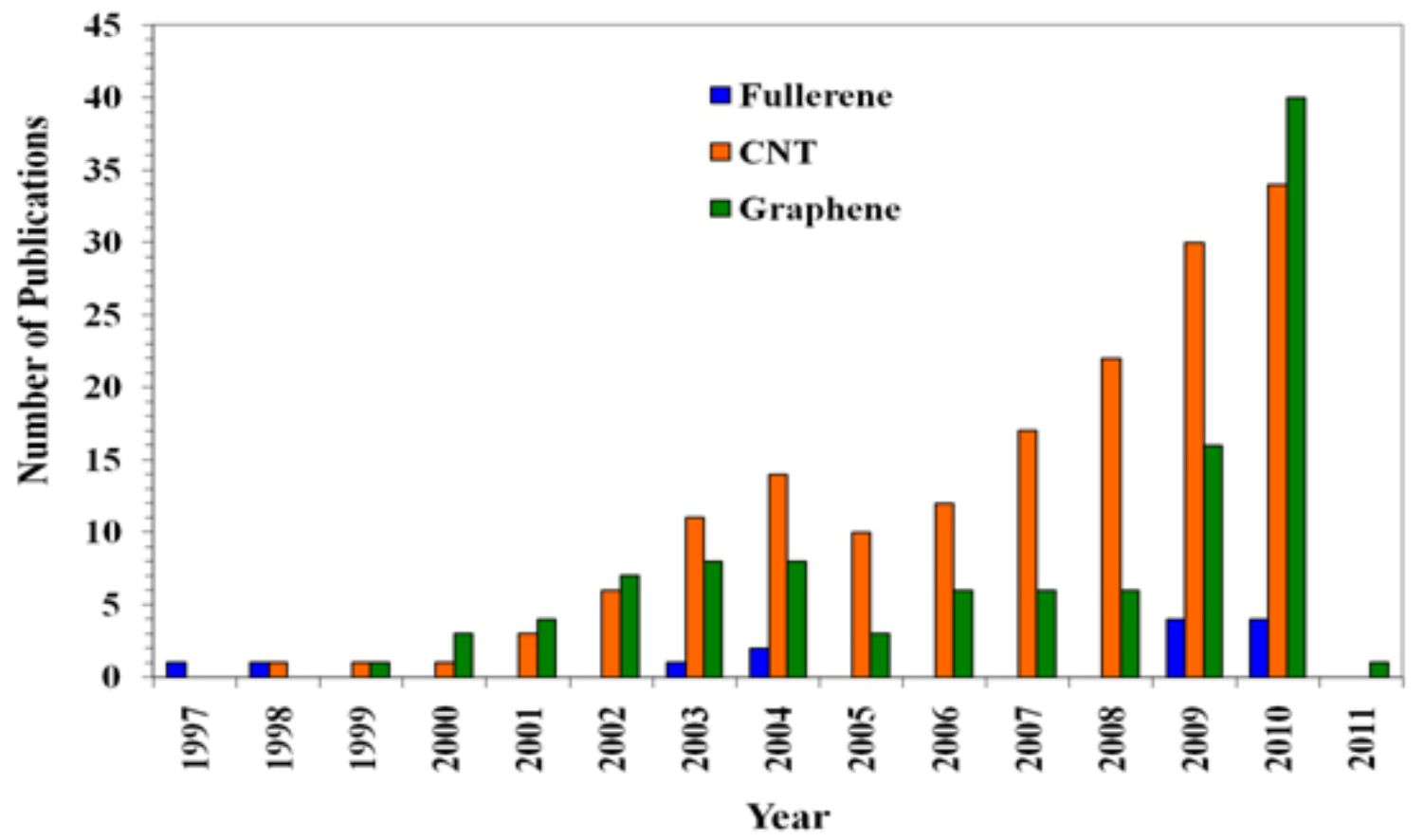

Figure 2.11: Publication trend involving carbon nanostructure as anode in Li-ion batteries. (Source: scopus.com)

Carbon nanotubes (CNT), carbon nanofibers (CNF) and graphene have been used in different forms as anode material in Li-ion batteries. Apart from forming the anode individually, these were used as composite with many other metals/oxides/other materials, sometimes even as encapsulated structures. Accordingly, the anode structures have shown a wide range of properties, some of them being impressive. Table 2.3 summarizes some of these important findings. As can be observed from figure 2.11, research with carbon nanostructures as anode in $\mathrm{Li}$-ion batteries have resulted in high volume of publications. In the following table, only a small fraction of them have been presented, with the aims to cover widest possible range of structures and properties. Details about each of these structures could be found in the corresponding references. 
Table 2.3: Summary of applying CNT/CNF/graphene as anode in Li-ion batteries

\begin{tabular}{|c|c|c|c|c|}
\hline $\begin{array}{l}\text { Sl. } \\
\text { No. }\end{array}$ & Material & $\begin{array}{l}\text { Reversible } \\
\text { Capacity } \\
\text { (mAh/g) }\end{array}$ & C-rate & Reference \\
\hline 1 & CNT & 125 & $0.02 \mathrm{C}$ & [114] \\
\hline 2 & CNT & 150 & $0.05 \mathrm{C}$ & [(115] \\
\hline 3 & CNT & 200 & $0.13 \mathrm{C}$ & [116] \\
\hline 4 & CNT & 280 & $0.11 \mathrm{C}$ & [117] \\
\hline 5 & CNT & 250 & $0.07 \mathrm{C}$ & [118] \\
\hline 6 & CNT & 436 & $0.11 \mathrm{C}$ & [119] \\
\hline 7 & CNT & 230 & $0.13 \mathrm{C}$ & [120] \\
\hline 8 & ONTC* & 515 & $0.5 \mathrm{~mA} \mathrm{~cm}^{-2}$ & [95] \\
\hline 9 & $\mathrm{CNT}-\mathrm{Sn}_{2} \mathrm{Sb}$ & 500 & $0.08 \mathrm{C}$ & [117] \\
\hline 10 & Sn-CNT & 550 & $0.13 \mathrm{C}$ & [120] \\
\hline 11 & Sb-CNT & 449 & $0.07 \mathrm{C}$ & [118] \\
\hline 12 & Bi-CNT & 309 & $0.07 \mathrm{C}$ & [118] \\
\hline 13 & SnNi-CNT & 500 & $0.13 \mathrm{C}$ & [120] \\
\hline 14 & SnSb-CNT & 480 & $100 \mathrm{~mA} \mathrm{~g}^{-1}$ & [121] \\
\hline 15 & $\begin{array}{l}\mathrm{SnO}_{2}-\mathrm{CNT} \\
\text { core-shell }\end{array}$ & 586 & $0.5 \mathrm{C}\left(336 \mathrm{~mA} \mathrm{~g}^{-1}\right)$ & [122] \\
\hline \multirow{2}{*}{16} & \multirow{2}{*}{$\begin{array}{l}\mathrm{CNT} @ \mathrm{SnO}_{2-} \\
\text { Au nanocable }\end{array}$} & 575 & $1500 \mathrm{~mA} \mathrm{~g}^{-1}$ & \multirow{2}{*}[123]{} \\
\hline & & 680 & $180 \mathrm{~mA} \mathrm{~g}^{-1}$ & \\
\hline 17 & Si-CNT & 940 & $0.156 \mathrm{~mA} \mathrm{~cm}^{-2}$ & [124] \\
\hline 18 & $\begin{array}{l}\text { Si-Graphite- } \\
\text { MWNT }\end{array}$ & 584 & $0.1 \mathrm{C}$ & [125] \\
\hline 19 & $\begin{array}{l}\text { Si-Graphite- } \\
\text { SWNT }\end{array}$ & 960 & $0.11 \mathrm{C}$ & [126] \\
\hline
\end{tabular}




\begin{tabular}{|c|c|c|c|c|}
\hline 20 & $\mathrm{CeO}_{2}-\mathrm{CNT}$ & 133 & NA & [127] \\
\hline 21 & CuO-CNT & 500 & $0.1 \mathrm{C}\left(67 \mathrm{~mA} \mathrm{~g}^{-1}\right)$ & {$[128]$} \\
\hline 22 & SWNT paper & 520 & $0.2 \mathrm{C}$ & [129] \\
\hline 23 & CNFs@CNT ${ }^{\#}$ & 400 & $0.2 \mathrm{C}$ & [130] \\
\hline 24 & Graphene & 540 & $0.13 \mathrm{C}$ & \multirow{3}{*}{ [131] } \\
\hline 25 & Graphene-CNT & 730 & $0.13 \mathrm{C}$ & \\
\hline 26 & Graphene-C60 & 784 & $0.13 \mathrm{C}$ & \\
\hline
\end{tabular}

* ONTC - Ordered nanostructured tin-based oxides/carbon composite

${ }^{\#} \mathrm{CNFs} @ \mathrm{CNT}$ - Carbon nanofibers encapsulated in carbon nanotubes

NA - Not available in the literature

From table 2.3 , it is clearly evident that most of the $\mathrm{CNT} / \mathrm{CNF} /$ graphene based anodes offer higher capacities than graphite electrode, but many of them were tested at low current rates. It will be interesting to know about their capacities at higher current rates. Moreover, other issues, like safety, also need to be addressed.

\subsubsection{Specialty Carbon Nanostructures}

Apart from the above-mentioned well-known carbon nanostructures, some special structures were synthesized and applied as anode for Li-ion batteries. Though research on these structures is in its infancy stage, but novelty of these structures deserves a mention.

\subsubsection{1 $\quad$ Ordered mesoporous and hierarchically porous carbon}

Ordered mesoporous carbon structure has extremely high surface area, which could successfully be used for lithium storage. Zhou et al. [132] reported such a structure prepared using ordered silica as a template and sucrose as precursor and it has shown one 
of the highest Li-ion intercalation capacities among carbon-based structures. This structure offered a reversible specific capacity of $1100 \mathrm{mAh} \mathrm{g}^{-1}$ (which corresponds to the structure $\mathrm{Li}_{3} \mathrm{C}_{6}$ ) at a current density of $100 \mathrm{~mA} \mathrm{~g}^{-1}$. Figure 2.12 presents a TEM image of the structure. This structure shows good promise for anodic application in Li-ion battery.

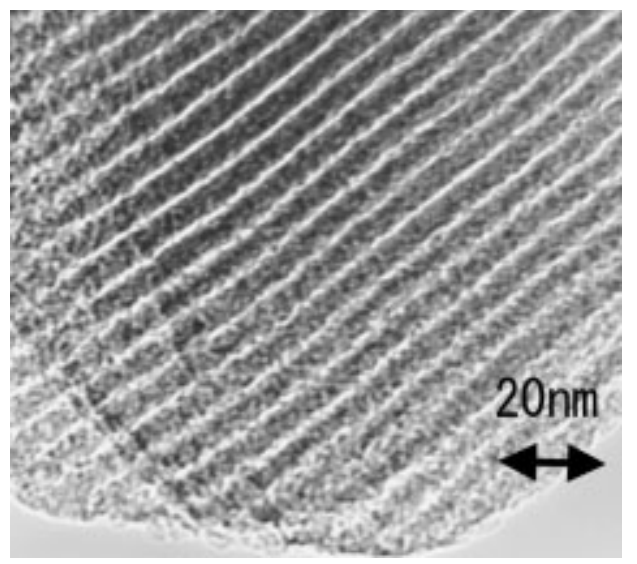

Figure 2.12: TEM image of the ordered mesoporous carbon structure, perpendicular to the direction of hexagonal pore arrangement. [132]

Another important and almost similar carbon morphology is hierarchically porous carbon, which contains a 3-diemnsional network of both meso- and macro-pores. This special structure also exhibited superior electrochemical properties for Li storage [133]. A representative SEM image, in figure 2.13 (a), gives an idea of the structure.
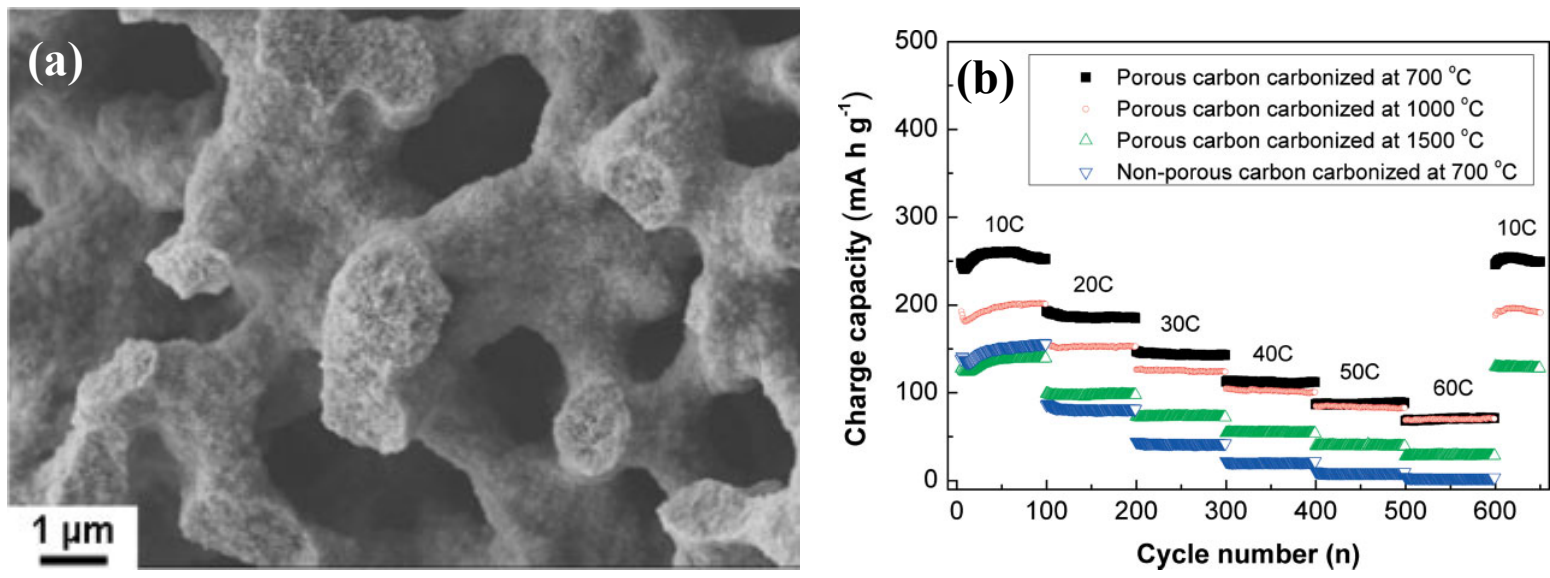

Figure 2.13: Hierarchically porous carbon structure - (a) SEM image of the structure and (b) rate capability of the anode. [133] 
This special structure offers a stable capacity of $\sim 500 \mathrm{mAh} \mathrm{g}^{-1}$, at $0.2 \mathrm{C}$ rate. However, the most interesting property of this structure is its rate capability; even at very high rate of $60 \mathrm{C}$, this anode has shown appreciable capacity. Advantage of this structure is the connection between large and small pores, which offers optimized conditions for electrolyte penetration.

\subsection{Superfine expanded graphite fiber}

One more special structure is superfine expanded graphite fibers, which consists of thin carbon ribbons and is characterized by high surface area as well as mesopores (in the range of 2-5 nm) [111]. This structure offered specific capacities of $1000 \mathrm{mAh} \mathrm{g}^{-1}$ at $0.1 \mathrm{C}$ rate, $700 \mathrm{mAh} \mathrm{g}^{-1}$ at $0.2 \mathrm{C}$ rate and an excellent rate performance, up to a high rate of 60C. Owing its excellent performance, this structure shows good promise for anodic application in Li-ion batteries.

\subsubsection{Scope of Improvement in Li-ion Batteries}

Information presented in the previous sections lead to summarize the shortcomings of the presently used/proposed anode materials and help to identify the need of the future. The very first issue that needs to be attended is enhancement of specific capacity of the anodes. Many anodes, involving carbon nanostructures, are not yet fully explored to gain excellent specific capacities. Moreover, rate performance and stability in longer cycles are two other major properties that need to be improved. Even the anodes with best specific capacities, e.g. Si nanowire anode, did not perform well 
when it comes to higher rate and/or more number of cycles. Capacity degradation per cycle should be minimized, in order to ensure good life of the anode.

Another important issue is binder. Commercial batteries and most of the research efforts (mainly involving C-nanostructures) have used polymeric binders in the anodes, adding a redundant weight, ultimately reducing the specific capacity of the electrode. Moreover, most widely used binder, poly(vinylidene fluoride) (PVdF), is known to react with graphitic materials and metallic lithium to form stable compounds [134-137]. Such reactions are highly exothermic; e.g. reaction with metallic Li produces $7.2 \mathrm{~kJ}$ of energy per gram of PVdF [135]. Thus, presence of binder could lead to thermal runaway, necessitating incorporation of additional safety features in the battery. Advanced Li-ion batteries, hence, need a binder-free electrode, to avoid such kind of capacity loss and inclusion of additional safety features.

In an effort to address all these issues, the present study proposes direct synthesis of interface-controlled MWCNTs on copper current collectors and their application as the anode in Li-ion cells. This kind of structure is expected to have many advantages over the conventional anodes. First of all, unlike all the past studies involving CNTs, which used raw CNTs and polymeric binders [114-116], the present structure will have directly grown CNTs on the current collector, thus avoiding the polymeric binders completely. In this way, it reduces harmful effect of the polymeric binder, reduces weight of the active material, increases specific capacity and shows potential to be used for high temperature application. Secondly, CNTs do not have any kind of expansion/contraction and pulverization problem (like $\mathrm{Si}$ and $\mathrm{SnO}_{2}$ ), so it should be able to sustain its capacity for long cycles. Third, due to direct growth on current collector, each CNT is well bonded to 
it, thus all of them contribute to the capacity. Fourth, high specific surface area of CNTs allows more Li-ion intercalation. Fifth, higher conductivity of the active anode material is important for achieving higher capacity [138]. In that respect, MWCNT, known to be excellent charge carriers, is a good candidate and aid in achieving higher capacity. Moreover, direct growth and strong bonding between the CNTs and substrate further helps in efficient charge transport. Further, the anode structure can be very easily fabricated using thermal chemical vapor deposition (CVD) process. With all these advantages, it is expected that the approach, proposed in this study, will lead to a better structure for Li-ion battery anodes.

\section{References}

1. Extended definition - field emission. Websters's online dictionary. [Online] [Cited: June 23, 2011.] http://www.websters-onlinedictionary.org/definitions/Field+Emission?cx=partner-pub0939450753529744\%3Av0qd01-tdlq\&cof=FORID\%3A9\&ie=UTF$8 \& \mathrm{q}=$ Field+Emission\&sa=Search $\# 922$.

2. W. Schottky. Über kalte und warme elektronenentladungen. 1923, Z. Phys., Vol. 14, pp. 63-106.

3. R.H. Fowler, L.W. Nordheim. Electron emission in intense electric fields. 1928, Proc. R. Soc. London, Vol. A119, pp. 173-181.

4. L.W. Nordheim. The effect of the image force on the emission and reflection of electrons by metals. 1928, Proc. R. Soc. London, Vol. A121, pp. 626-639.

5. O.W. Richardson, K.T. Compton. The photoelectric effect. 1912, Science, Vol. 35, pp. 783-784.

6. O.W. Richardson. The laws of photoelectric action and the unitary theory of light (Lichtquanten Theorie). 1912, Science, Vol. 36, pp. 57-58.

7. O.W. Richardson. The emission of electrons from tungsten at high temperatures: An experimental proof that the electric current in metals is carried by electrons. 1913, Science, Vol. 38, pp. 57-61. 
8. A. Modinos. Field, thermionic and secondary electron emission spectroscopy. Plenum Press, New York, 1984.

9. E.L. Murphy, R.H. Good, Jr. Thermionic emission, field emission and the transition region. 1956, Phys. Rev., Vol. 102, pp. 1464-1473.

10. R.G. Forbes. Field-induced electron emission from electrically nanostructured heterogeneous (ENH) materials. 2001, Ultramicroscopy, Vol. 89, pp. 7-15.

11. R.G. Forbes. Low-Macroscopic-Field Electron Emission from Carbon Films and other Electrically Nanostructured Heterogeneous Materials: Hypotheses about Emission Mechanism. 2001, Solid State Electron., Vol. 45, pp. 779-808.

12. J. Robertson. Electron field emission from diamond and diamond-like carbon for field emission diaplays. 5, 1999, Carbon, Vol. 37, pp. 759-763.

13. J. Robertson. Mechanisms of electron field emission from diamond, diamond-like carbon, and nanostructured carbon. 2, 1999, J. Vac. Sci. Technol. B, Vol. 17, pp. 659-665.

14. L.W. Swanson, L.C. Crouser. Anomalous toal energy distribution for a tungsten field emitter. 10, 1966, Phys. Rev. Lett., Vol. 16, pp. 389-392.

15. E.W. Plummer, J.W. Gadzuk. Surface states on tungsten. 1970, Phys. Rev. Lett., Vol. 25, pp. 1493-1495.

16. C.D. Ehrlich, E.W. Plummer. Measurement of the absolute tunneling current density in field emission from tungsten. 1978, Phys. Rev. B, Vol. 18, pp. 3767-3771.

17. L.W. Swanson. Current fluctuations from various crystal faces of a clean tungsten field emitter. 1978, Surf. Sci., Vol. 70, pp. 165-180.

18. T. Engel, R. Gomer. Adsorption of $\mathrm{CO}$ on tungsten: Field emission from single planes. 6, 1969, The J. Chem. Phys., Vol. 50, pp. 2428-2437.

19. T. Engel, R. Gomer. Adsorption of oxygen on tungsten: Field emission from single planes. 4, 1970, The J. Chem. Phys., Vol. 52, pp. 1832-1841.

20. M.J.G. Lee. Field emission of hot electrons from tungsten. 1973, Phys. Rev. Lett., Vol. 30, pp. 1193-1196.

21. Y.-H. Lee, C.-H. Choi, Y.-T. Jang, E.-K. Kim, B.-K. Ju, N.-K. Min, J.-H. Ahn. Tungsten nanowires and their field electron emission properties. 2002, App. Phys. Lett., Vol. 81, pp. 745-747. 
22. R. Seelaboyina, J. Huang, J. Park, D.H. Kang, W. Choi. Multistage field enhancement of tungsten oxide nanowires and its field emission in various vacuum conditions. 2006, Nanotechnology, Vol. 17, pp. 4840-4844.

23. C. Lea. Field emission from carbon fibres. 1973, J. Phys. D: Appl. Phys., Vol. 6, pp. 1105-1114.

24. N. Kumar. Method of forming field emitter device with diamond emission tips. 5199918 USA, 1993.

25. K. Okano, K. Hoshina, M. Iida, S. Koizumi, T. Inuzuka. Fabrication of a diamond field emitter array. 1994, Appl. Phys. Lett., Vol. 64, pp. 2742-2744.

26. W. Zhu, G.P. Kochanski, S. Jin, L. Seibles. Defect-enhanced electron field emission from chemical vapor deposited diamond. 1995, J. Appl. Phys., Vol. 78, pp. 27072711.

27. A.A. Talin, L.S. Pan, K.F. McCarty, T.E. Felter, H.J. Doerr, R.F. Bunshah. The relationship between the spatially resolved field emission characteristics and the raman spectra of a nanocrystalline diamond cold cathode. 1996, Appl. Phys. Lett., Vol. 69, pp. 3842-3844.

28. I. Lahiri, S.-W..Oh, J.Y. Hwang, S. Cho, Y.-K.. Sun, R. Banerjee, W. Choi. High capacity and excellent stability of lithium ion battery anode using interfacecontrolled binder-free multiwall carbon nanotubes grown on copper. 2010, ACS Nano, Vol. 4, pp. 3440-3446.

29. R. Seelaboyina, I. Lahiri, W. Choi. Carbon-nanotube-embedded novel threedimensional alumina microchannel cold cathodes for high electron emission. 2010, Nanotechnology, Vol. 21, p. 145206.

30. W.A. de Heer, A. Châtelain, D. Ugarte. A carbon nanotube field-emission electron source. 1995, Science, Vol. 270, pp. 1179-1180.

31. W.B. Choi, D.S. Chung, J.H. Kang, H.Y. Kim, Y.W. Jin, I.T. Han, Y.H. Lee, J.E. Jung, N.S. Lee, G.S. Park, J.M. Kim. Fully sealed, high-brightness carbonnanotube field-emission display. 20, 1999, Appl. Phys. Lett., Vol. 70, pp. 31293131 .

32. X. Xu, G.R. Brandes. A method for fabricating large-area, patterned, carbon nanotube field emitters. 17, 1999, Appl. Phys. Lett., Vol. 74, pp. 2549-2551.

33. M.J. Fransen, Th. L. van Rooy, P. Kruit. Field emission energy distributions from individual multiwalled carbon nanotubes. 1999, Appl. Surf. Sci., Vol. 146, pp. $312-$ 327. 
34. K.A. Dean, B.R. Chalamala. The environmental stability of field emission from single-walled carbon nanotubes. 19, 1999, Appl. Phys. Lett., Vol. 75, pp. 30173019.

35. Y. Saito, S. Uemura. Field emission from carbon nanotubes and its application to electron sources. 2000, Carbon, Vol. 38, pp. 169-182.

36. R.B. Sharma, V.N. Tondare, D.S. Joag, A. Govindaraj, C.N.R. Rao. Field emission from carbon nanotubes grown on a tungsten tip. 2001, Chem. Phys. Lett., Vol. 344, pp. 283-286.

37. K.B.K. Teo, M. Chhowalla, G.A.J. Amaratunga, W.I. Milne, G. Pirio, P. Legagneux, F. Wyczisk, D. Pribat, D.G. Hasko. Field emission from dense, sparse, and patterned arrays of cacrbon nanofibers. 2002, Appl. Phys. Lett., Vol. 80, pp. 2011-2013.

38. N. de Jonge, Y. Lamy, K. Schoots, T.H. Oosterkamp. High brightness electron beam from a multi-walled carbon nanotube. 2002, Nature, Vol. 420, pp. 393-396.

39. D. Liu, S. Zhang, S.-E. Ong, G. Benstetter, H. Du. Surface and electron emission properties of hydrogen-free diamon-like carbon flms investigated by atomic force microscopy. 2006, Mater. Sci. Engg. A, Vol. 426, pp. 114-120.

40. R. Seelaboyina, J. Huang, W.B. Choi. Enhanced field emission of thin multiwall carbon nanotubes by electron multiplication from microchannel plate. 2006, Appl. Phys. Lett., Vol. 88, p. 194104.

41. R. Seelaboyina, S. Boddepalli, K. Noh, M. Jeon, W. Choi. Enhanced field emission from aligned multistage carbon nanotube emitter arrays. 2008, Nanotechnology, Vol. 19, p. 065605.

42. I. Lahiri, R. Seelaboyina, J.Y. Hwang, R. Banerjee, W. Choi. Enhanced Field Emission from Multi-Walled Carbon Nanotubes Grown on Pure Copper Substrate. 2010, Carbon, Vol. 48, pp. 1531-1538.

43. G. Eda, H.E. Unalan, N. Rupesinghe, G.A.J. Amaratunga, M. Chhowalla. Field emission from graphene based composite thin films. 2008, Appl. Phys. Lett., Vol. 93, p. 233502.

44. A. Malesevic, R. Kemps, A. Vanhulsel, M. Pal Chowdhury, A. Volodin, C. V. Haesendonck. Field emission from vertically aligned few-layer graphene. 2008, J. Appl. Phys., Vol. 104, p. 084301.

45. S. Watcharotone, R.S. Ruoff, F.H. Read. Possibilities for graphene for field emission: modeling studies using the BEM. 2008, Physics Procedia, Vol. 1, pp. 7175. 
46. Z.-S. Wu, S. Pei, W. Ren, D. Tang, L. Gao, B. Liu, F. Li, C. Liu, H.-M. Cheng. Field emission of single-layer graphene films prepared by electrophoretic deposition. 2009, Adv. Mater., Vol. 21, pp. 1756-1760.

47. M. Qian, T. Feng, H. Ding, L. Lin, H. Li, Y. Chen, Z. Sun. Electron field emission from screen-printed graphene films. 2009, Nanotechnology, Vol. 20, p. 425702.

48. Z. Xiao, J. She, S. Deng, Z. Tang, Z. Li, J. Lu, N. Xu. Field electron emission characteristics and physical mechanism of individual single-layer graphene. 2010, ACS Nano, Vol. 4, pp. 6332-6336.

49. S.W. Lee, S. S. Lee, E.-H. Yang. A study on field emission characteristics of planar graphene layers obtained from a highly oriented pyrolyzed graphite block. 2009, Nanoscale Res. Lett., Vol. 4, pp. 1218-1221.

50. R.N. Thomas, R.A. Wickstrom, D.K. Schroder, H.C. Nathanson. Fabrication and some applications of large-area silicon field emission arrays. 1974, Solid-State Electronics, Vol. 17, pp. 155-163.

51. C.A. Spindt, I. Brodie, L. Humphrey, E.R. Westerberg. Physical properties of thinfilm field emission cathodes with molybdenum cones. 1976, J. Appl. Phys., Vol. 47, pp. 5248-5263.

52. Y.B. Li, Y. Bando, D. Golberg, K. Kurashima. Field emission from MoO3 nanobelts. 2002, Appl. Phys. Lett., Vol. 81, pp. 5048-5050.

53. V.N. Tondare, C. Balasubramanian, S.V. Shende, D.S. Joag, V.P. Godbole, S.V. Bhoraskar, M. Bhadbhade. Field emission from open ended aluminum nitride nanotubes. 2002, Appl. Phys. Lett., Vol. 80, pp. 4813-4815.

54. P. Dorozhkin, D. Golberg, Y. Bando, Z.-C. Dong. Field emission from individual BC-N nanotube rope. 2002, Appl. Phys. Lett., Vol. 81, pp. 1083-1085.

55. J. Chen, S.Z. Deng, N.S. Xu, W. Zhang, X. Wen, S. Yang. Temperature dependence of field emission from cupric oxide nanobelt films. 2003, Appl. Phys. Lett., Vol. 83, pp. 746-748.

56. Y.J. Chen, Q.H. Li, Y.X. Liang, T.H. Wang, Q. Zhao, D.P. Yu. Field-emission from long SnO2 nanobelt arrays. 2004, Appl. Phys. Lett., Vol. 85, pp. 5682-5684.

57. Y.W. Zhu, H.Z. Zhang, X.C. Sun, S.Q. Feng, J. Xu, Q. Zhao, B. Xiang, R.M. Wang, D.P. Yu. Efficient field emission from ZnO nanoneedle arrays. 2003, Appl. Phys. Lett., Vol. 83, pp. 144-146.

58. S.H. Jo, D. Banerjee, Z.F. Ren. Field emission of zinc oxide nanowires grown on carbon cloth. 2004, Appl. Phys. Lett., Vol. 85, pp. 1407-1409. 
59. R.C. Wang, C.P. Liu, J.L. Huang, S.-J. Chen, Y.-K. Tseng, S.-C. Kung. ZnO nanopencils: Efficient field emitters. 2005, Appl. Phys. Lett., Vol. 87, p. 013110.

60. Q. Zhao, H.Z. Zhang, Y.W. Zhu, S.Q. Feng, X.C. Sun, J. Xu, D.P. Yu. Morphological effects on the field emission of $\mathrm{ZnO}$ nanorod arrays. 2005, Appl. Phys. Lett., Vol. 86, p. 203115.

61. E.E. Windsor. Construction and performance of practical field emitters from lanthanum hexaboride. 1969, Proc. IEEE, Vol. 116, pp. 348-350.

62. K.C. Qi, Z.L. Lin, W.B. Chen, G.C. Cao, J.B. Cheng, X.W. Sun. Formation of extremely high current density LaB6 field emission arrays via e-beam deposition. 2008, Appl. Phys. Lett., Vol. 93, p. 093503.

63. H. Zhang, J. Tang, J. Yuan, J. ma, N. Shinya, K. Nakajima, H. Murakami, T. Ohkubo, L.-C. Qin. Nanostructured LaB6 field emitter with lowest apical work function. 2010, Nano Lett., Vol. 10, pp. 3539-3544.

64. S. Lee, D.Y. Jeon. Effect of degassed elements on the degradation behavior of carbon nanotube cathodes in sealed field emission-backlight units. 2006, Appl. Phys. Lett., Vol. 88, p. 063502.

65. M. Kumar, T. Okazaki, M. Hiramatsu, Y. Ando. The use of camphor-grown carbon nanotube array as an efficient field emitter. 2007, Carbon, Vol. 45, pp. 1899-1904.

66. Y. S. Choi, J. H. Kang, H. Y. Kim, B. G. Lee, C. G. Lee, S. K. Kang, Y. W. Jin, J. W. Kim, J. E. Jung, J. M. Kim. A simple structure and fabrication of carbonnanotube field emission display . 2004, Appl. Surf. Sci., Vol. 221, pp. 370-374.

67. M.S. Chung, B.-G. Yoon. Analysis of the slope of the Fowler-Nordheim plot for field emission from n-type semiconductors. 2003, J. Vac. Sci. Technol. B, Vol. 21, pp. 548-551.

68. J. Zhang, X. Wang, W. Yang, W. Yu, T. Feng, Q. Li, X. Liu, C. Yang. Interaction between Carbon Nanotubes and Substrate and its Implication in Field Emission Mechanism. 2006, Carbon, Vol. 44, pp. 418-422.

69. D.G. Walker, W. Zhang, T.S. Fisher. Simulation of field emitted electron trajectories and transport from carbon nanotubes. 2004, J. Vac. Sci. Technol. B, Vol. 22, pp. 1101-1107.

70. Sinha, D. Roy Mahapatra, J.T.W. Yeow, R. Melnik. Modeling the field emission current fluctuation in carbon nanotube thin films. N. 2007. NSTI-Naotech 2007, Vol. 1. pp. 76-79. ISBN 1420061828. 
71. G. Chen, W. Wang, J. Peng, C. He, S. Deng, N. Xu, Z. Li. Screening effects on field emission from arrays of $(5,5)$ carbon nanotubes: Quantum mechanical simulations. 2007, Phys. Rev. B, Vol. 76, p. 195412.

72. J.M. Bonard, C. Klinke, K.A. Dean, B.F. Coll. Degradation and failure of carbon nanotube field emitters. 2003, Phys. Rev. B, Vol. 67, p. 115406.

73. P.H. Cutler, N.M. Miskovsky, P.B. Lerner, M.S. Chung.The use of internal field emission to inject electronic charge carriers into the conduction band of diamond films: a review. 1999, Appl. Surf. Sci. , Vol. 146, pp. 126-33.

74. Q. Ngo, D. Petranovic, S. Krishnan, A.M. Cassell, Q. Ye, J. Li, M. Meyyappan, C.Y. Yang. Electron transport through metal-multiwall carbon nanotube interfaces. 2004, IEEE Trans. Nanotechnol. , Vol. 3, pp. 311-317.

75. N.S. Xu, R.V. Latham, Y. Tzeng. Field-dependence of the area-density of cold electron emission sites on broad-area CVD diamond films. 1993, Electron. Lett. , Vol. 29, pp. 1596-97.

76. B.S. Satyanarayana, A. Hart, W.I. Milne, J. Robertson. Field emission from tetrahedral amorphous carbon. 1997, Appl. Phys. Lett., Vol. 71, pp. 1430-32.

77. P.M. Ryan, A.S. Verhulst, D. Cott, A. Romo-Negreira, T. Hantschel, J.J. Boland. Optimization of multi-walled carbon nanotube-metal contacts by electrical stressing . 2010, Nanotechnology, Vol. 21, p. 045705.

78. Z. Chen, Q. Zhang, P. Lan, B. Zhu, T. Yu, G. Cao, D. den Engelsen. Ultrahighcurrent field emission from sandwich-grown well-aligned uniform multi-walled carbon nanotube arrays with high adherence strength. 2007, Nanotechnology, Vol. 18, p. 265702 .

79. Y.D. Lee, K.-S. Lee, Y.-H. Lee, B.-K. Ju. Field emission properties of carbon nanotube film using a spray method. 2007, Appl. Surf. Sci., Vol. 254, pp. 513-516.

80. S. Talapatra, S. Kar, S.K. Pal, R. Vajtai, L. Ci, P. Victor, M.M. Shaijumon, S. Kaur, O. Nalamasu, P.M. Ajayan. Direct growth of aligned carbon nanotubes on bulk metals . 2006, Nature Nanotech., Vol. 1, pp. 112-116.

81. M. Dubosc, S. Casimirius, M.-P. Besland, C. Cardinaud, A. Granier, J.-L. Duvail, A. Gohier, T. Minéa, V. Arnal, J. Torres. Impact of the Cu-based substrates and catalyst deposition techniques on carbon nanotube growth at low temperature by PECVD . 2007, Microelectron. Eng., Vol. 84, pp. 2501-2505.

82. X. Yin, Q. Wang, C. Lou, X. Zhang, W. Lei. Growth of multi-walled CNTs emitters on an oxygen-free copper substrate by chemical-vapor deposition. 2008, App.1 Surf. Sci., Vol. 254, pp. 6633-6636. 
83. J. Zhang, X. Wang, W. Yang, W. Yu, T. Feng, Q. Li, X. Liu, C. Yang. Interaction between carbon nanotubes and substrate and its implication on field emission mechanism. 2006, Carbon, Vol. 44, pp. 418-422.

84. Y. Matsuda, W.-Q. Deng, W.A.III. Goddard. Contact Resistance Properties between Nanotubes and Various Metals from Quantum Mechanics. 2007, J. Phys. Chem. C, Vol. 111, pp. 11113-11116.

85. M. Parthangal, R.E. Cavicchi, M.R. Zachariah. A Generic Process of Growing Aligned Carbon Nanotubes Arrays on Metals and Metal Alloys. P2007, Nanotechnology, Vol. 18, pp. 185605(1-5).

86. O.V. Yazyev, A. Pasquarello. Effect of metal elements in catalytic growth of carbon nanotubes. 2008, Phys. Rev. Lett., Vol. 100, p. 156102.

87. G.A. Nazri, G. Pistoia. Lithium Batteries: Science and Technology. Springer Science+Business Media, New York, 2009.

88. B.L. Ellis, K.T. Lee, L.F. Nazar. Positive electrode materials for Li-ion and Libatteries. 2010, Chem. Mater., Vol. 22, pp. 691-714.

89. T. Brousse, D. Defives, L. Pasquereau, S.M. Lee, U. Herterich, D.M. Schleich. Metal oxide anodes for Li-ion batteries. 1997, Ionics, Vol. 3, pp. 332-337.

90. V. Manev, I. Naidenov, B. Puresheva, P. Zlatilova, G. Pistoia. Electrochemical Performance of Natural Brazilian Graphite as Anode Material for Lithium-Ion Rechargeable Cells. 1995, J. Power Sources, Vol. 55, pp. 211-215.

91. B.A. Boukamp, G.C. Lesh, R.A. Huggins. All-Solid Lithium Electrodes with MixedConductor Matrix. 1981, J. Electrochem. Soc., Vol. 128, pp. 725-729.

92. S.-M. Paek, E.-J. Yoo, I. Honma. Enhanced Cyclic Performance and Lithium Storage Capacity of SnO2/Graphene Nanoporous Electrodes with ThreeDimensionally Delaminated Flexible Structure. 2009, Nano Lett., Vol. 9, pp. 72-75.

93. C.K. Chan, H. Peng, G. Liu, K. McIlwrath, X.F. Zhang, R.A. Huggins, Y. Cui. High-Performance Lithium Battery Anodes Using Silicon Nanowires. 2008, Nature Nanotechol., Vol. 3, pp. 32-35.

94. C.K. Chan, R. Ruffo, S.S. Hong, Y. Cui. Surface chemistry and morphology of the solid electrolyte interphase on silicon nanowire ilthium-ion battery anodes. 2009, J. Power Sources, Vol. 189, pp. 1132-1140.

95. J. Fan, T. Wang, C. Yu, B. Tu, Z. Jiang, D. Zhao. Ordered, Nanostructured TinBased Oxides/Carbon Composite as the Negative-Electrode Material for LithiumIon Batteries. 2004, Adv. Mater, Vol. 16, pp. 1432-1436. 
96. C.K. Chan, X.F. Zhang, Y. Cui. High capacity Li ion battery anodes using Ge nanowires. 2008, Nano Lett., Vol. 8, pp. 307-309.

97. J. Graetz, C.C. Ahn, R. Yazami, B. Fultz. Nanocrystalline and thin film germanium electrodes with High lithium capacity and high rate capabilities. 2004, J. Electrochem. Soc., Vol. 151 , pp. A698-A702.

98. H. Lee, H. Kim, S.-G. Doo, J Cho. Synthesis and optimization of nanoparticle Ge confined in a carbon matrix for lithium battery anode material. J. Electrochem. Soc., Vol. 154, pp. A343-A346.

99. H. Lee, J. Cho. Sn78Ge22@carbon core-shell nanowires as fast and high-capacity lithium storage media. 2007, Nano Lett., Vol. 7, pp. 2638-2641.

100. K.T. Nam, D.-W. Kim, P.J. Yoo, C.-Y. Chiang, N. Meethong, P.T. Hammond, Y.M. Chiang, A.M. Belcher. Virus-enabled synthesis and assembly of nanowires for lithium ion battery electrodes. 2006, Science, Vol. 312, pp. 885-888.

101. C.K. chan, R. Ruffo, S.S. Hong, R.A. Huggins, Y. Cui. Structural and electrochemical study of the reaction of lithium with silicon nanowires. 2009, J. Power Sources, Vol. 189, pp. 34-39.

102. F. Belliard, P.A. Connor, J.T.S. Irvine. Novel tin oxide-based anodes for Li-ion batteries. 2000, Solid State Ionics, Vol. 135, pp. 163-167.

103. Y. Idota, T. Kubota, A. Matsufuji, Y. Maekawa, T. Miyasaka. Tin-based amorphous oxide: a high-capacity lithium-ion-storage material. 1997, Science, Vol. 276, pp. 1395-1397.

104. P. Poizot, S. Laruelle, S. Grugeon, L. Dupont, J.-M. Tarascon. Nano-sized transition-metal oxides as negative-electrode materials for lithium-ion batteries. 2000, Nature, Vol. 407, pp. 496-499.

105. P. Limthongkul, H. Wang, Y.-M. Chiang. Nanocomposite Li-ion battery anodes produced by the partial reduction of mixed oxides. 2001, Chem. Mater., Vol. 13, pp. 2397-2402.

106. G.X. Wang, Y. Chen, K. Konstantinov, M. Lindsay, H.K. Liu, S.X. Dou. Investigation of cobalt oxides as anode materials for Li-ion batteries. 2002, J. Power Sources, Vol. 109, pp. 142-147.

107. N. Du, H. Zhang, B.D. Chen, J.B. Wu, X.Y. Ma, Z.H. Liu, Y. Zhang, D. Yang, X. Huang, J. Tu. Porous Co3O4 nanotubes derived from Co4(CO) 12 clusters on carbon nanotube templates: a highly efficient material for Li-battery applications. 2007, Adv. Mater., Vol. 19, pp. 4505-4509. 
108. A.C. Dillon, A.H. Mahan, R. Deshpande, P.A. Parilla, K.M. Jones, S.-H. Lee. Metal oxide nano-particles for improved electrochromic and lithium-ion battery technologies. 2008, Thin Solid Films, Vol. 516, pp. 794-797.

109. S.-H. Lee, Y.-H. Kim, R. Deshpande, P.A. Parilla, E. Whitney, D.T. Gillaspie, K.M. Jones, A.H. Mahan, S. Zhang, A.C. Dillon. Reversible lithium-ion insertion in molybdenum oxide nanoparticles. 2008, Adv. Mater., Vol. 20, pp. 3627-3632.

110. C.K. Chan, H. Peng, R.D. Twesten, K. Jarausch, X.F. Zhang, Y. Cui. Fact compleletly reversible Li insertion in vanadium pentoxide nanoribbons. 2007, Nano Lett., Vol. 7, pp. 490-495.

111. N.A. Kaskhedikar, J. Maier. Lithium Storage in Carbon Nanostructures. 2009, Adv. Mater., Vol. 21, pp. 2664-2680.

112. M.S. Dresselhaus, G. Dresselhaus, P.C. Eklund. Science of fullerenes and carbon nanotubes. Academic Press, San Diego, USA, 1996.

113. R.O. Loufty, S. Katagiri. Fullerene materials for lithium-ion battery applications. [ed.] E. Osawa.Kluwer Academic Publishers, Dordrecht, The Netherlands, 2002.

114. Z.-H. Yang, H.-Q. Wu. Electrochemical Intercalation of Lithium into Raw Carbon Nanotubes. 2001, Mater Chem. Phys., Vol. 71, pp. 7-11.

115. E. Frackowiak, F. Béguin. Electrochemical Storage of Energy in Carbon Nanotubes and Nanostructured Carbons. 2002, Carbon, Vol. 40, pp. 1775-1787.

116. H.-C. Shin, M. Liu, B. Sadanadan, A. M. Rao. Electrochemical Insertion of Lithium into Multi-Walled Carbon Nanotubes Prepared by Catalytic Decomposition. 2002, J. Power Sources, Vol. 112, pp. 216-221.

117. W.X. Chen, J.Y. Lee, Z. Liu. Electrochemical Lithiation and De-Lithiation of Carbon Nanotube-Sn2Sb Nanocomposites. 2002, Electrochem. Comm., Vol. 4, pp. 260-265.

118. Y. NuLi, J. Yang, M. Jiang. Synthesis and characterization of Sb/CNT and Bi/CNT composites as anode materials for lithium-ion batteries. 2008, Mater. Lett., Vol. 62, pp. 2092-2095.

119. M. Wang, Z.H. Li, G.T. Wu. Electrochemical lithium insertion properties of carbon nanotubes produced by catalytic pyrolysis of acetylene. 2005, Russ. J. Electrochem., Vol. 41, pp. 946-949.

120. Z.P.Guo, Z.W.Zhao, H.K. Liu, S.X. Dou. Electrochemical lithiation and delithiation of MWNT-Sn/SnNi nanocomposites. 2005, Carbon, Vol. 43, pp. 13921399. 
121. M.S. Park, S.A. Needham, G.-X. Wang, Y.-M. Kang, J.-S. Park, S.-X. Dou, H.-K. Liu. Nanostructured SnSb/Carbon Nanotube Composites Synthesized by Reductive Precipitation for Lithium-Ion Batteries. 2007, Chem. Mater., Vol. 19, pp. 24062410.

122. Y. Wang, H. C. Zeng, J. Y. Lee. Highly Reversible Lithium Storage in Porous SnO2 Nanotubes with Coaxially Grown Carbon Nanotube Overlayers. 2006, Adv. Mater., Vol. 18, pp. 645-649.

123. G. Chen, Z. Wang, D. Xia. One-Pot Synthesis of Carbon Nanotube@SnO2-Au Coaxial Nanocable for Lithium-Ion Batteries with High Rate Capability. 2008, Chem. Mater., Vol. 20, pp. 6951-6956.

124. J. Shu, H. Li, R. Yang, Y. Shi, X. Huang. Cage-Like Carbon Nanotubes/Si Composite as Anode Material for Lithium Ion Batteries. 2006, Electrochem. Comm., Vol. 8, pp. 51-54.

125. Y. Zhang, X. G. Zhang, H. L. Zhang, Z. G. Zhao, F. Li, C. Liu, H.M. Cheng. Composite Anode Material of Silicon/Graphite/Carbon Nanotubes for Li-Ion Batteries. 2006, Electrochimica Acta, Vol. 51, pp. 4994-5000.

126. W.Wang, P. N. Kumta. Reversible High Capacity Nanocomposite Anodes of Si/C/SWNTs for Rechargeable Li-Ion Batteries. 2007, J. Power Sources, Vol. 172, pp. 650-658.

127. C. Li, N.Sun, J. Ni, J. Wang, H. Chu, H. Zhou, M. Li. Y. Li. Controllable Preparation and Properties of Composite Materials Based on Ceria Nanoparticles and Carbon Nanotubes. 2008, J. Solid State Chem., Vol. 181, pp. 2620-2625.

128. S.-F. Zheng, J.-S. Hu, L.-S. Zhong, W.-G. Song, L.-J. Wan, Y.-G. Guo. Introducing Dual Functional CNT Networks into CuO Nano/Micro Spheres toward Superior Electrode Materials for Lithium-Ion Batteries. 2008, Chem. Mater., Vol. 20, pp. 3617-3622.

129. B.J. Landi, M.J. Ganter, C.M. Schauerman, C.D. Cress, R.P. Raffaelle. Lithium Ion Capacity of Single Wall Carbon Nanotube Paper Electrodes. 2008, J. Phys. Chem. C, Vol. 112, pp. 7509-7515.

130. J. Zhang, Y.-S. Hu, J.-P. Tessonnier, G. Weinberg, J. Maier, R. Schlogl, D.S. Su. CNFs@CNTs: Superior carbon for electrochemical energy storage. 2008, Adv. Mater., Vol. 20, pp. 1450-1455.

131. E.J. Yoo, J. Kim, E. Hosono, H.-S. Zhoi, T. Kudo, I. Honma. Large Reversible Li Storage of Graphene Nanosheet Families for Use in Rechargeable Lithium Ion Batteries. 2008, Nano Lett., Vol. 8, pp. 2277-2282. 
132. H. Zhou, S. Zhu, M. Hibino, I. Honma, M. Ichihara. Lithium storage in ordered mesoporous carbon (CMK-3) with high reversible specific energy capacity and good cycling performance. 2003, Adv. Mater., Vol. 15, pp. 2107-2111.

133. Y.-S. Hu, P. Adelhelm, B. M. Smarsly, S. Hore, M. Antonietti, J. Maier. Synthesis of hierarchically porous carbon monoliths with highly ordered microstructure and their application in rechargeable lithium batteries with high-rate capability. 2007, Adv. Func. Mater., Vol. 17, pp. 1873-1878.

134. A. Guerfi, M. Kaneko, M. Petitclerc, M. Mori, K. Zaghib. LiFePO4 Water-Soluble Binder Electrode for Li-ion Batteries. 2007, J. Power Sources, Vol. 163, pp. 10471052.

135. S.S. Zhang, T.R. Jow. Study of Poly(Acrylonitrile-Methyl Methacrylate) as Binder for Graphite Anode and LiMn2O4 Cathode of Li-Ion Batteries. 2002, J. Power Sources, Vol. 109, pp. 422-426.

136. S.S. Zhang, K. Xu, T.R. Jow. Evaluation on a Water-Based Binder for the Graphite Anode of Li-Ion Batteries. 2004, J. Power Sources, Vol. 138, pp. 226-231.

137. E.P. Roth, D.H. Doughty, J. Franklin. DSC Investigation of Exothermic Reactions Occurring at Elevated Temperatures in Lithium-Ion Anodes Containing PVDFBased Binders. 2004, J. Power Sources, Vol. 134, pp. 222-234.

138. T. Ishihara, M. Nakasu, M. Yoshio, H. Nishiguchi, Y. Takita. Carbon Nanotube Coating Silicon Doped with Cr as a High Capacity Anode. 2005, J. Power Sources, Vol. 146, pp. 161-165. 


\section{MATERIALS AND METHODS}

This chapter presents a detailed description of growth of carbon nanotubes, samples preparation methods for field emitter and battery, and the characterization techniques followed in this study to explain structure and properties of these devices. Figure 3.1 presents an overall schematic about plan of research. Detailed description of each step is given in the following sections.

\subsection{Carbon Nanotube Synthesis}

Carbon nanotubes (CNT) were synthesized in the present study through thermal chemical vapor deposition (CVD) system. During the study of field emission, three different metals $(\mathrm{Cu}, \mathrm{Al}$ and $\mathrm{W})$ and one ceramic, low temperature co-fired ceramic (LTCC) were selected as the substrate materials. Field emission behavior of the CNTs grown on these substrates was compared with those synthesized on Si substrates. Reason for such substrate selection will be discussed in section 4.1. However, for the Li-ion battery purpose, CNTs were grown on Cu substrate only.

$500 \mu \mathrm{m}$ thick pure $\mathrm{Cu}, \mathrm{Al}, \mathrm{W}, \mathrm{Si}$ and LTCC sheets (with surface roughness $<10$ $\mu \mathrm{m})$ were taken as the substrate materials and all the substrates were first cut into required sizes. For field emission study, the sample size was $10 \mathrm{~mm} \times 10 \mathrm{~mm}$, while for battery study, the sample size is $\phi 14 \mathrm{~mm}$ disc. Cut samples were then cleaned thoroughly in acetone, methanol and de-ionized water and further dried in pressurized $\mathrm{N}_{2}$ gas. This process was performed to remove any surface contaminants from the surface of the 


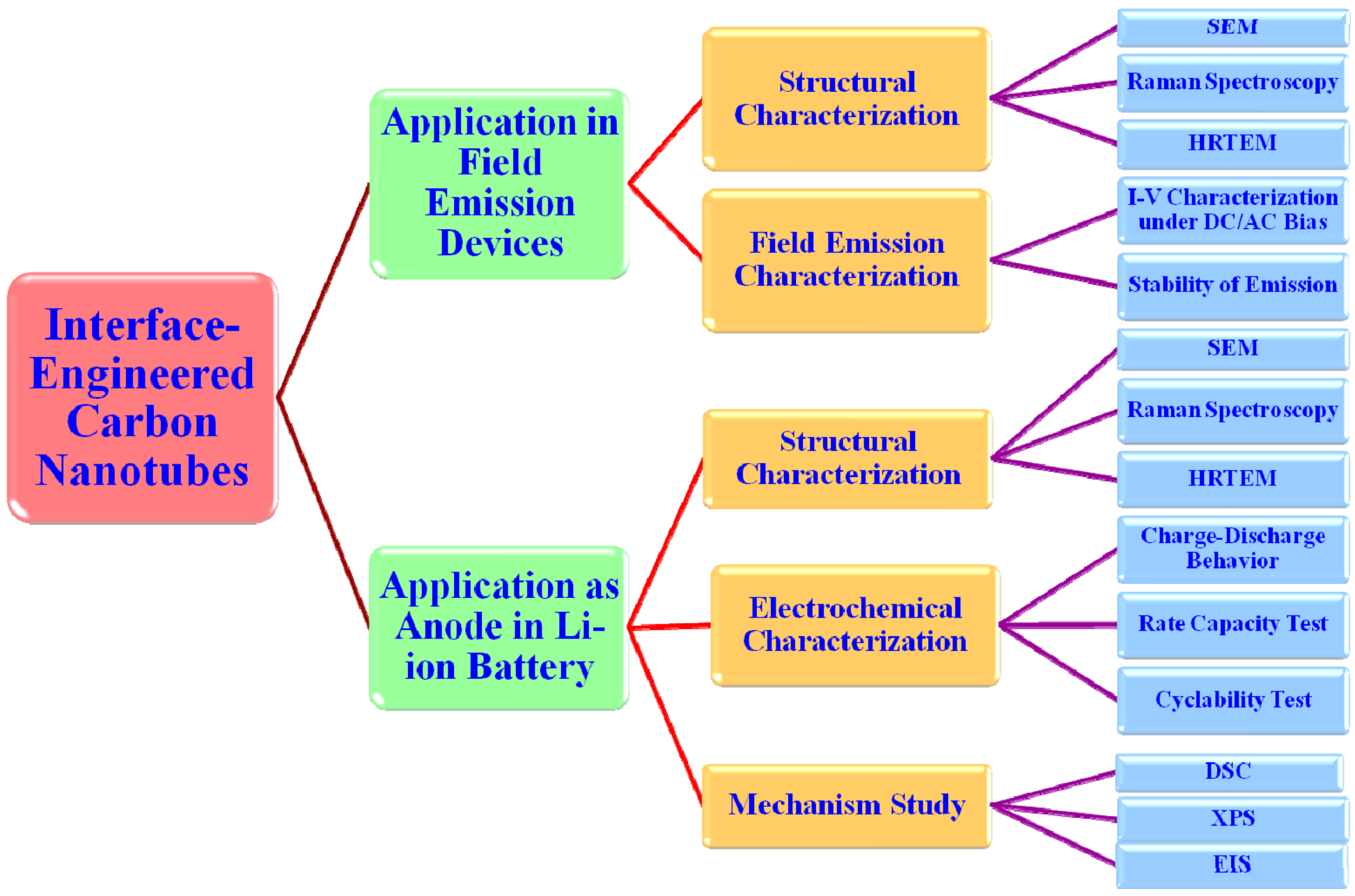

Figure 3.1: A brief schematic presentation of the present research work. 
samples. $\mathrm{Cu}$ has a tendency to form oxides when stored for long time. So, $\mathrm{Cu}$ samples were given an extra hot acetic acid treatment, prior to the cleaning step, in order to remove any surface oxide.

These cleaned samples were then introduced into a magnetron sputtering system (AJA International). Thin layers $(10-15 \mathrm{~nm}$, each) of interface layer (Ti or $\mathrm{Al})$ and catalyst (Ni or Fe) were sputtered on the samples. During sputtering, which is basically a physical vapor deposition process, base pressure was kept at $\sim 1 \times 10^{-7}$ Torr. For generating the plasma or ignition of sputtering gun, continuous flow $10 \mathrm{sccm}$ of Ar gas was maintained and chamber pressure was raised to $\sim 25 \mathrm{mTorr}$. Power of $\sim 75 \mathrm{~W}$ was applied to the magnetron guns. High concentration of $\mathrm{Ar}$ gas and $\sim 75 \mathrm{~W}$ of power was good enough for initiating the plasma. After generating plasma, the pressure was lowered down to $\sim 5$ mTorr, which was the deposition pressure. The samples were kept at room temperature during sputtering.

Thin-film sputtered samples were then inserted in a thermal CVD chamber (Easytube1000, FirstNano). All the CVD cycles were computer controlled. Before initiation of CVD, the system was used to be heated at $1173 \mathrm{~K}$ in presence of oxygen, followed by slow cooling, to ensure oxidation of any residue carbon from the previous cycle. During heating of the samples to the growth temperature, Ar gas was flown (@) $1000 \mathrm{sccm}$ ) to maintain an inert atmosphere within the furnace. CVD was performed at temperature of $923-973 \mathrm{~K}$, using $\mathrm{H}_{2}+\mathrm{C}_{2} \mathrm{H}_{4}$ (1:2 ratio) mixture as the precursor for $\mathrm{CNT}$ growth. After the growth period, samples were slowly cooled within the furnace, under an inert (Ar) gas envelope. All CNT growth cycles, reported in this study, were performed at atmospheric pressure condition. Information related to carbon nanotube 
growth could be found in the following references [1-4]. Figure 3.2 presents a schematic of the process flow chart.

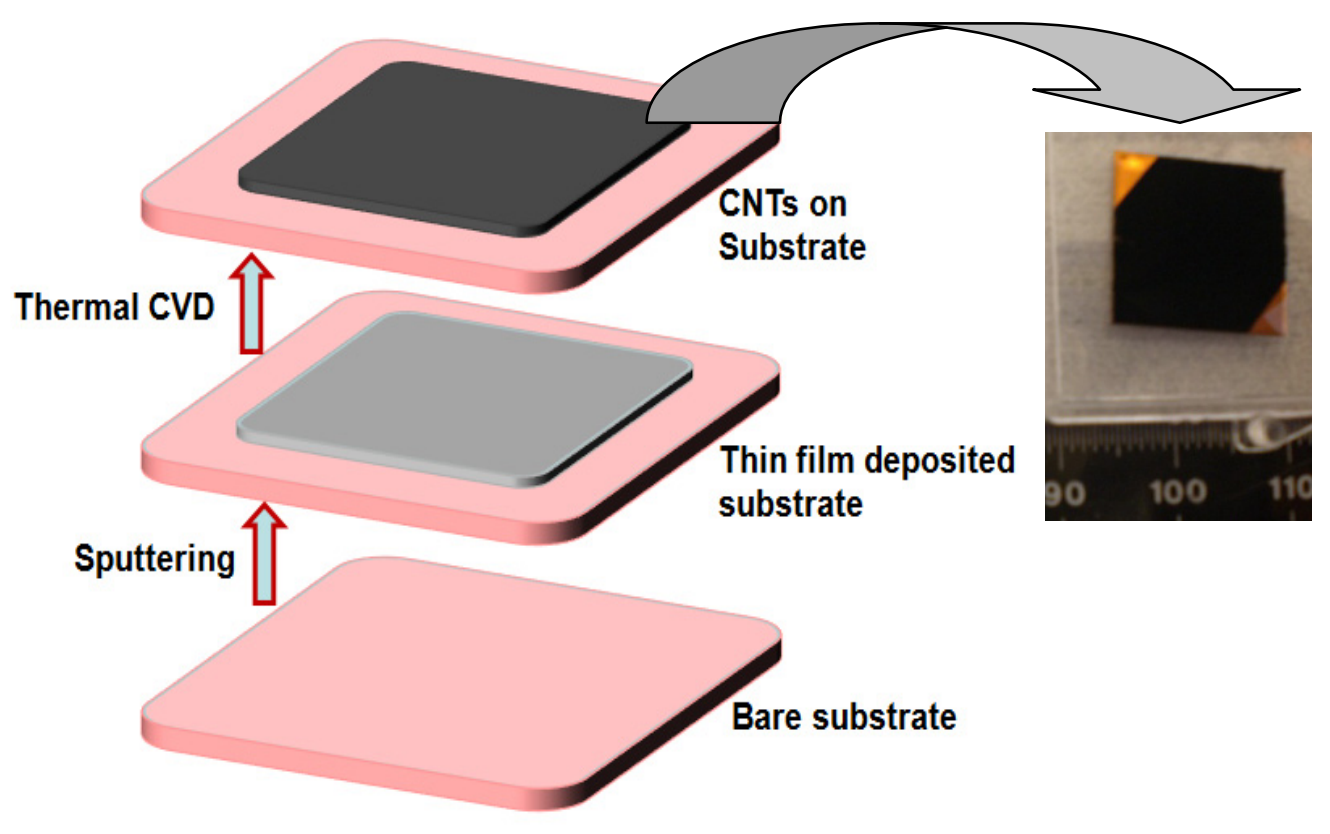

Figure 3.2: Schematic process flow-chart for synthesis of MWCNTs on various samples.

CVD growth results in formation of thick mat type randomly oriented multi walled carbon nanotubes (MWCNT) on the substrates. Figure 3.3 presents representative SEM images of the structure.

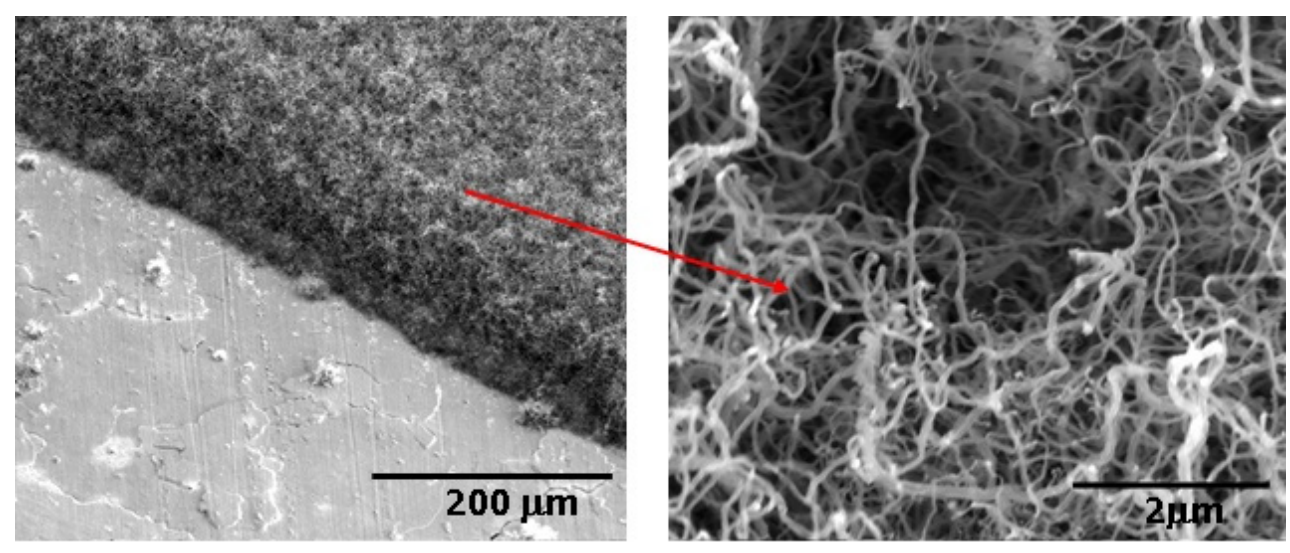

Figure 3.3: SEM images of the MWCNT structure, grown by CVD. 


\subsection{Preparation of Electrodes and Test Devices}

During the present study, CNT based structures were used as cathodes in field emission test set-ups, while similar structures acted as anodes for Li-ion batteries. Depending upon the application, preparation the electrodes and the test devices followed different routes. Details about these sample preparation methods are given below.

\subsubsection{Preparation of Conventional 2-Dimensional Field Emitters}

As-synthesized CNT samples, as described in section 3.1, were directly used as cathode of the field emitter test devices. In most cases, a flattened $\mathrm{Cu}$ sheet was used as the anode of the device. Field emission tests involving capture of field emission images used to have a green phosphor coated indium tin oxide (ITO) conducting glass piece as the anode. Inter-electrode distance was maintained at $1000 \mu \mathrm{m}$, by using alumina spacer materials. The device assembly was mounted on a $10 \mathrm{~mm}$ thick Teflon piece to avoid any short circuit or grounding with the field emission test chamber. Details about the electrical connection and other parameters of field emission test will be explained in section 3.4 .

\subsubsection{Preparation of 3-Dimensional Field Emitters}

Preparation of 3-D field emitters involved making micro-channels within $\mathrm{Cu}$ sheets by electro-discharge machining (EDM). Initially, $250 \mu \mathrm{m}$ thick copper sheet was cut into required size and micro-channels, of diameter $140 \pm 10 \mu \mathrm{m}$, were formed within the samples by EDM technique. Distance between each circle was maintained in a similar and repetitive manner for all samples. The samples, with micro-channels created in them, 
were later treated in the same way for CNT growth, as mentioned in section 3.1. It may be mentioned here that areas surrounding the micro-channels, except for a square area of $1 \mathrm{~mm}^{2}$ covering all the channels, were masked during catalyst deposition and hence, that area does not see any CNT growth during CVD. This step was performed to ensure that CNTs are synthesized only a very small lateral surface area and within the microchannels only. Figure 3.4 presents a schematic of the process flow chart to prepare the 3 D cathodes of field emission test device.

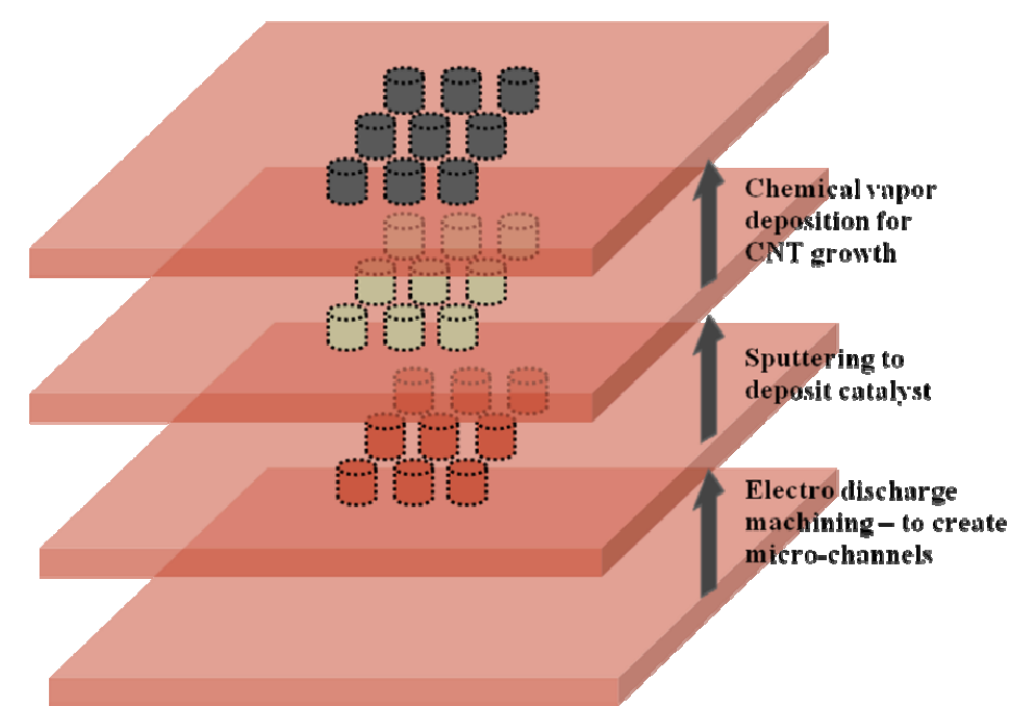

Figure 3.4: A schematic of the processing steps to fabricate the 3D CNT-based field emitter on Cu substrate.

\subsubsection{Preparation of Transparent Flexible Cathode for Field Emitters}

The process of preparation of transparent flexible field emission device involves several steps: graphene synthesis, transfer of graphene on polymeric substrate, anode 
preparation and cathode preparation. The following sub-sections will cover these process steps separately.

\subsubsection{Graphene Synthesis}

Graphene film was synthesized by CVD method, over $\mathrm{Cu}$ foils (Nimrod Copper Company). Large area $\mathrm{Cu}$ foils (thickness $\sim 50 \mu \mathrm{m}$ ) were cut into pieces of required dimensions and were annealed for $1 \mathrm{~h}$, at $1273 \mathrm{~K}\left(1000^{\circ} \mathrm{C}\right)$ and under $\mathrm{H}_{2}$ and $\mathrm{Ar}$ (flow ratio 1:5) environment, at $1 \mathrm{~atm}$ pressure, with an aim to increase the grain size to 500$1000 \mu \mathrm{m}$. After annealing, $\mathrm{Cu}$ foils were subjected to chemical etching at $333 \mathrm{~K}\left(60^{\circ} \mathrm{C}\right)$ using $1 \mathrm{M}$ acetic acid, to remove any oxide film generated over $\mathrm{Cu}$ foil during annealing. After washing the $\mathrm{Cu}$ foils with de-ionized (DI) water to remove acid trace from the surface, these were inserted at center of quartz tube in a thermal CVD system for graphene growth. Initially quartz tube was flushed with $\operatorname{Ar}(500 \mathrm{sccm})$ gas for 30 minutes to remove air from the quartz tube. Temperature of the furnace was raised up to $1273 \mathrm{~K}$ $\left(1000^{\circ} \mathrm{C}\right)$, at a rate of $100 \mathrm{~K} /$ minute, under $\operatorname{Ar}(500 \mathrm{sccm})$ and $\mathrm{H}_{2}(50-100 \mathrm{sccm})$ atmosphere. At the graphene synthesis temperature, $\mathrm{CH}_{4}(10-25 \mathrm{sccm})$ and $\mathrm{H}_{2}(50-100$ $\mathrm{sccm}$ ) gas was introduced into the CVD chamber for graphene growth. After 5 minutes of growth time, furnace was cooled to room temperature under $500 \mathrm{sccm}$ of Ar atmosphere. Whole CVD process was done at 1 atm pressure. Related information about the graphene growth and sample preparation may be found in references $[5,6]$.

\subsubsection{Transferring Graphene on PET}

Large area graphene film was transferred from $\mathrm{Cu}$ foils to Polyethylene terephthalate (PET) substrate using hot press lamination and chemical etching process. 
For hot press lamination, PET film along with graphene over $\mathrm{Cu}$ foil was passed through indigenously developed hot press rollers. This hot press laminated PET/graphene/Cu sheet was floated over the concentrated $\mathrm{FeCl}_{3}$ solution for chemical etching of $\mathrm{Cu}$ foil. Chemical etching was performed at room temperature for $\sim 2 \mathrm{~h}$ which completely dissolved the $\mathrm{Cu}$. Graphene/PET film was washed into the de-ionized (DI) water and dried in air.

\subsubsection{Cathode Fabrication}

For fabricating the hybrid graphene/CNT cathode, multiwall carbon nanotube (MWCNT) solution was spin coated over graphene/PET substrate. MWCNT solution was prepared in polyvinyl alcohol (PVA) and DI water mixture. Approximately, $4 \mathrm{wt} \%$ of PVA (with average molecular weight $\left(M_{\mathrm{w}}\right)$ of $88,000-97,000$ and $98-99 \%$ hydrolyzed, Alfa Aesar) was mixed into DI water and the resulting mixture was stirred continuously at $333 \mathrm{~K}\left(60^{\circ} \mathrm{C}\right)$, until PVA was completely dissolved into water, forming a clear solution. After cooling down the solution to room temperature, $\sim 1 \mu \mathrm{g}$ of MWCNT (ILJIN Nanotech Co. Ltd) was added into it and dispersed by using high power ultra-sonication tip. MWCNT in PVA/DI water makes a very homogeneous and stable solution. Spin coating of MWCNT solution at $1000 \mathrm{rpm}$ for $30 \mathrm{~s}$ produces a homogeneous and transparent coating of CNT emitters over graphene/PET film.

\subsubsection{Anode Fabrication}

Anode screen was prepared by dip coating of graphene/PET substrate in green phosphor solution. Green phosphor powder (Phosphor Tech) was mixed into the DI water. Solution was ultrasonicated which created suspension of phosphor particle in the 
water. Graphene/PET film was dipped into the solution multiple times for uniform phosphor coating. Details about anode fabrication could be found in reference [6].

\subsubsection{Preparation of Li-ion Battery Test Cells}

All tests related to performance evaluation of CNT based structure as anode in Liion batteries, were carried out in half-cell mode. For this purpose, CNTs grown on $\mathrm{Cu}$ discs of $14 \mathrm{~mm}$ diameter were used. Samples were weighed before and after CVD growth to calculate the weight of CNTs.

In order to characterize electrochemical behavior of the structure as a proposed anodic material in Li-ion batteries, typical coin cells (half-cell) were prepared. The coin cells were prepared in a CR2032 press (Hohsen Corp., Japan). The complete cell preparation steps were performed in an argon glove box (Unilab Mbrann), maintaining the oxygen and humidity level (both individually $<0.1 \mathrm{ppm}$ ) within the chamber. A pure Li (purity - 99.9\%) metal foil (150 $\mu \mathrm{m}$ thick) was used as the reference and counter electrode, while the MWCNT-on-Cu was used as the working electrode. All the coin cells used 1.0M $\mathrm{LiPF}_{6}$ in EC-DEC (1:1 in volume) (ethylene carbonate - diethyl carbonate) as the electrolyte and a typical polypropylene-polyethylene material (Celgard 3401) as the separator.

\subsection{Structural Characterization}

Several characterization techniques were used for understanding structural details of the MWCNT structures, as described below. 


\subsubsection{Scanning Electron Microscopy (SEM)}

JEOL JSM-7000F ${ }^{\mathrm{TM}}$ field emission scanning electron microscope (FE-SEM), operated at $3 \mathrm{kV}$, was used for the characterization of CNTs. During SEM observation, samples were sometimes given a tilt up to $45^{\circ}$, to clearly observe a top-view image of the structures. Most of the samples, except low temperature co-fired ceramics (LTCC) samples, did not require any conducting coating during SEM observation, since the samples themselves were conducting. For LTCC samples, a conductive $\mathrm{Cu}$ tape was used to avoid excessive charging during SEM.

\subsubsection{Transmission Electron Microscopy (TEM)}

Transmission electron microscopy (TEM) was mainly used to observe CNTs at very high resolution, in order to understand more details about their structures. For this purpose, samples were prepared by dispersing CNTs (scraped out from the samples) on $\mathrm{Cu}$ grids. High resolution transmission electron microscopy (HRTEM) was used to observe the interface between $\mathrm{Cu}$ substrate and CNTs, in the form of selective area diffraction patterns (SADP) and lattice fringe images. For the purpose of interface observation, site-specific sample preparation technique, using FEI Nova 200 NanoLab dual beam focused ion beam (FIB), has been implemented. The area of interest has been protected by Pt layer deposition, in order to minimize the Gallium damage during sample preparation. The region of interest was sectioned and milled using gallium ion beam and lifted out. The sample, with dimensions $10 \times 5 \times 2 \mu \mathrm{m}$, was attached to a copper-TEM grid. Additional thinning and cleaning using FIB, till $60 \mathrm{~nm}$ thickness, was consequently performed at $30 \mathrm{KeV}$ and $5 \mathrm{KeV}$, respectively, to remove the redeposition and ion beam 
damage. The site specific sample has been characterized using FEI TECHNAI F20 ${ }^{\mathrm{TM}}$ field emission TEM, operating at $200 \mathrm{kV}$.

\subsubsection{Stereological Analysis}

Stereological analysis was carried out by ImageJ software [7], which is public domain image processing program developed at the National Institute of Health. Using this software, quantitative analysis of the diameter distribution and density of CNTs was performed using multiple SEM images. Further, quantification of lattice fringe images was also done by this software.

\subsubsection{X-Ray Diffraction}

X-ray diffraction (XRD) studies were performed using in a Siemens D-500 ${ }^{\mathrm{TM}} \mathrm{X}$ ray diffractometer, operating at $40 \mathrm{kV}$ and $40 \mathrm{~mA}$, and using $\mathrm{Cu} \mathrm{K}_{\alpha}(\lambda=1.542 \AA)$ radiation. A scan rate of $0.2^{\circ} / \mathrm{min}$ and a step size of $0.01^{\circ}$ have been used. The phases present in the samples were identified using the JCPDS standard database.

\subsubsection{Raman Spectroscopy}

Raman spectroscopy is one of the important characterization tools that was used to gain knowledge about structures of CNTs. It is well-known technique for characterization of CNTs. Raman spectra of various CNT based samples were obtained by using argon ion $\left(\mathrm{Ar}^{+}\right)$laser system (Spectra Physics, model 177G02) of wavelength $514.5 \mathrm{~nm}$. Backscattered spectra were collected by high throughput holographic imaging 
spectrograph (Kaiser Optical Systems, model HoloSpec $f / 1.8 \mathrm{i}$ ) with volume transmission grating, holographic notch filter and charge coupled device detector (Andor Technology). Raman system had a spectral resolution of $4 \mathrm{~cm}^{-1}$ and the spectra were collected at an exposure time of $300 \mathrm{~s}$.

\subsection{Field Emission Characterization}

Field emission characteristics of the samples were tested at a vacuum level of $10^{-7}$ torr. In the diode configuration, used for field emission tests, CNT grown on different substrates was made cathode and a flattened $\mathrm{Cu}$ sheet was used as anode. Electrical connections of the test device are shown schematically in figure 3.5.

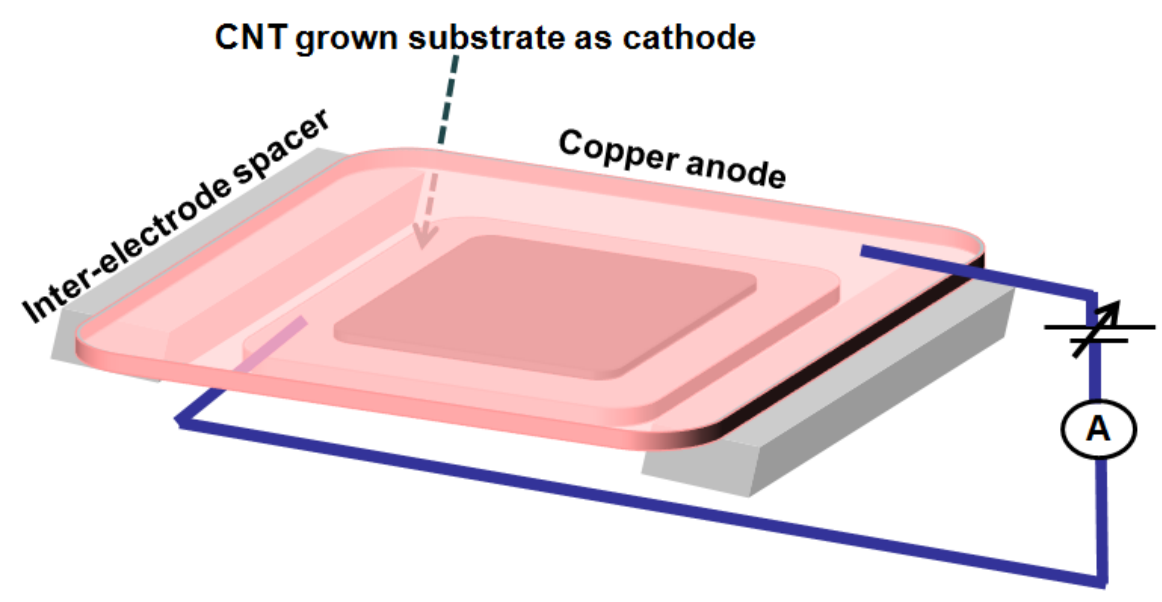

Figure 3.5: A schematic of field emission test set-up (for better visual effect, transparency has been added to the anode of this schematic - in reality, anode is a solid copper sheet).

For direct current (DC) voltage and current measurements, Keithley 248 high voltage supply and 2010 digital multimeter, respectively, were used. In the pulsed mode, 
field was generated by an Agilent function generator (model 33220A), coupled with a Trek high voltage amplifier (model 20/20C) and the current was monitored through a PEARSON current monitor (model 4100, having 1.0 V/A), attached with an Agilent oscilloscope (model MSO6034A). Frequency of the pulsed signal was kept at $400 \mathrm{~Hz}$ and a sine-wave function was applied through the function generator.

\subsection{Evaluation of Battery Performance}

The charge-discharge behavior of the coin cells were characterized in TOSCAT $3100 \mathrm{U}^{\mathrm{TM}}$ multi-channel battery testing unit, at room temperature, in galvanostatic mode. The instrument was programmed to read in each $10 \mathrm{mV}$ step. The half cells were cycled in the voltage range $2.0-0.01 \mathrm{~V}$ - at a very slow rate $(0.1 \mathrm{C})$ during the initial formation process and at different rates in the following cycles. Information about testing of batteries can also be found in references $[8,9]$.

\subsection{Other Characterization Techniques}

Apart from using these main characterization techniques to understand structure and properties of CNT based devices, some other specialized techniques were also used with different aims. Differential scanning calorimetry (DSC) was used to understand safety issue of the batteries at higher temperature, while electrochemical impedance spectroscopy (EIS) and x-ray photoelectron spectroscopy (XPS) were mainly focused to understand the mechanism of charge transfer through CNT-electrolyte interface. Nanoscratch tests were performed to quantify CNT-substrate bonding energy at nano-scale. 


\subsubsection{Differential Scanning Calorimetry (DSC)}

Differential scanning calorimetry (DSC) experiments were conducted in a Du Pont DSC $2910^{\mathrm{TM}}$ calorimeter on various samples. Since, this technique was used to understand safety of Li-ion half cells, utmost precaution was taken during handling of the samples. The cycled cells were disassembled in an argon glove box, all CNTs were scraped from the current collector and were sealed hermetically in plastic sample boxes. Sample boxes were opened near DSC equipment, quickly transferred on to the aluminum pans and the lid was closed to enforce Ar atmosphere. Before ramping, all the samples were equilibrated at room temperature, $303 \mathrm{~K}\left(30^{\circ} \mathrm{C}\right)$. The scan rate was maintained at $10 \mathrm{~K} \min ^{-1}$. After data acquisition, samples were cooled slowly to room temperature. All DSC tests were performed under Ar atmosphere, to mimic the actual cell conditions (which were prepared within Ar glove box and sealed).

\subsubsection{Electrochemical Impedance Spectroscopy (EIS)}

Electrochemical impedance spectroscopy (EIS) studies were carried out in a Gamry Reference $3000^{\mathrm{TM}}$ Potentiostat/Galvanostat/ZRA instrument. For all the half cells studies, MWCNT-on-Cu side was made as working electrode, while the pure Li metal side acted as both counter and reference electrode. The tests were performed in galvanostatic mode, using an $\mathrm{AC}$ current signal of $10 \mu \mathrm{A}(\mathrm{rms})$ amplitude. Data were recorded for the frequency range of $100 \mathrm{kHz}$ to $1 \mathrm{mHz}$. Acquired data was analyzed using Echem Analyst software, in order to generate Nyquist and Bode plots. 


\subsubsection{X-ray Photoelectron Spectroscopy (XPS)}

The X-ray photoelectron spectroscopy (XPS) analysis for the CNT samples was

performed using a Versa Probe ${ }^{\mathrm{TM}} 5000$ instrument (Physical Electronics, Chanhassen, $\mathrm{MN}$ ) for the surface elemental analysis. A monochromatic Al K $\alpha$ X-ray beam source at $1486.6 \mathrm{eV}$ and 49.3 $\mathrm{W}$ power was used for XPS scanning. The spot size of X-ray was 200 $\mu \mathrm{m}$ and the sample was sputter cleaned and operated at $4.5 \times 10^{-8}$ torr vacuum pressure. The energy resolution of the survey scan was $1.0 \mathrm{eV}$.

The depth profile studies using the XPS from the surface have been conducted using the $\mathrm{Al} \mathrm{K} \alpha \mathrm{X}$-ray source. The samples were kept under the Ar-atmosphere capsule to minimize the exposal time to air. The takeoff angle of the X-ray was $45^{\circ}$ and the beam diameter was $200 \mu \mathrm{m}$ and no charge neutralization was used in this experiment. Ar ion was used for the sputtering of the depth profile with a beam energy of $1 \mathrm{kV}$ and $0.5 \mu \mathrm{A}$. The high resolution scans of C 1s, O 1s, F 1s, Li 1s, and P 2p energy spectra were used for the peak identification with pass-energy of $23.5 \mathrm{eV}$ and energy resolution of $0.1 \mathrm{eV}$. The hydrocarbon peak with $284.8 \mathrm{eV}$ was used for peak calibration. Two different depth profile rates were used for the samples - one using $2 \mathrm{kV}, 2 \mu \mathrm{A}$ (with about $32 \AA / \mathrm{min}$ rate) and the other was operated $1 \mathrm{kV}, 0.5 \mu \mathrm{A}$ (with about $7 \AA / \mathrm{min}$ ). The etching rates were calibrated on Si sample and hence, actual rates on CNT samples may vary.

\subsubsection{Nano-Scratch}

Hysitron Triboindenter ${ }^{\mathrm{TM}}$ (Hysitron Inc., Minneapolis, MN, USA) with $100 \mathrm{~nm}$ Berkovich pyramidal tip, is used in 2D scratch mode for nano-scratch studies, using a 
normal load of $150 \mu \mathrm{N}$. Scratches were initiated from a bare surface and continued for a length $15 \mu \mathrm{m}$ (see figure 3.6 for a schematic of the scratch test). Scratch profiles are obtained by scanning probe microscopy (SPM) with the same tip at a set point load of 2 $\mu$ N. Analysis of the SPM images was performed by Scanning Probe Image Processor (SPIP) version 4.5.1 (Image Metrology, Denmark) software (10).

\section{Berkovich}

\section{Indenter}

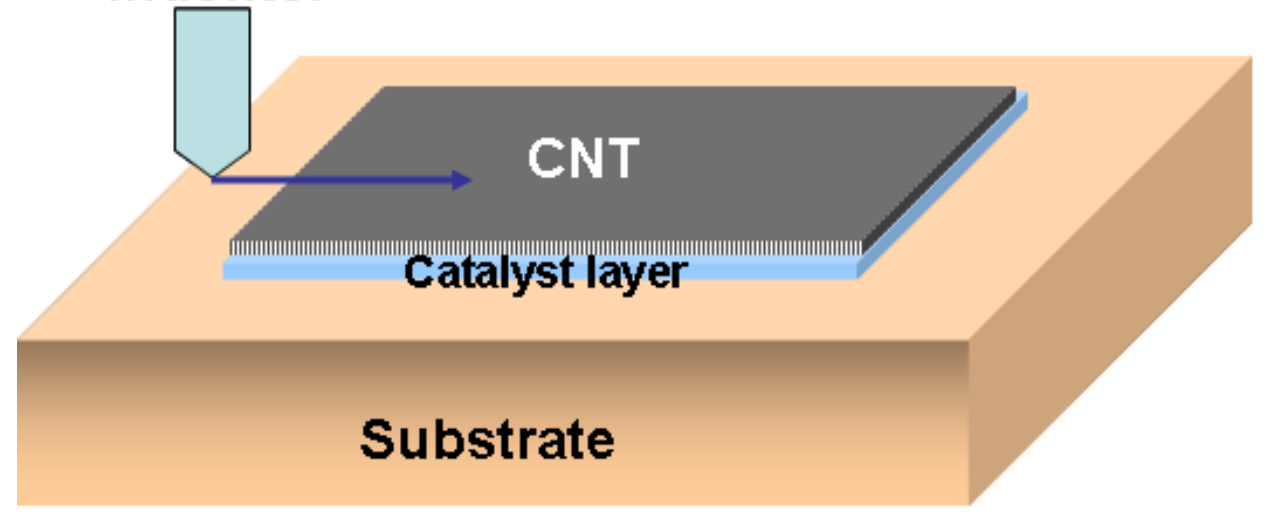

Figure 3.6: Schematic of the nano-scratch method, followed in the present study. A normal load of $150 \mu N$ was used for all the scratch tests.

Response of the nano-scratch tests, in the form of lateral force vs. lateral displacements are given in appendix-1. Geometric formulae were used to calculate bonding energy of single CNTs with substrate, from these plots. Details of calculation procedure are given in appendix-2 [11]. 


\section{References}

1. I. Lahiri, R. Seelaboyina, J.Y. Hwang, R. Banerjee, W. Choi. Enhanced field emission from multi-walled carbon nanotubes grown on pure copper substrate. 2010, Carbon, Vol. 48, pp. 1531-1538.

2. I. Lahiri, R. Seelaboyina, W. Choi. [ed.] Y.K. Yap. Boston, Field emission response from multiwall carbon nanotubes grown on different metallic substrates. 2010. Mater. Res. Soc. Symp. Proc. Vol. 1204, pp. 1204-K18-21.

3. R. Seelaboyina, I. Lahiri, W. Choi. Carbon-nanotube-embedded novel threedimensional alumina microchannel cold cathodes for high electron emission. 2010, Nanotechnology, Vol. 21, p. 145206.

4. I. Lahiri, W. Choi. Interface control: A modified rooting technique for enhancing field emission from multiwall carbon nanotube based bulk emitters. 2011, Acta Mater., Vol. 59, pp. 5411-5421.

5. I. Lahiri, V.P. Verma, W. Choi. An all-graphene based transparent and flexible field emission device. 2011, Carbon, p. DOI: 10.1016/j.carbon.2010.12.044.

6. V.P. Verma, S. Das, I. Lahiri, W. Choi. Large-area graphene on polymer film for flexible and transparent anode in field emission device. 2010, Appl. Phys. Lett., Vol. 96, p. 203108.

7. M.D. Abramoff, P.J. Magelhaes, S.J. Ram. Image processing with ImageJ. 2004, J. Biophotonics Intl., Vol. 11, pp. 36-42.

8. I. Lahiri, S.-W. Oh, J.Y. Hwang, S. Cho, Y.-K. Sun, R. Banerjee, W. Choi. High capacity and excellent stability of lithium ion battery using interface-controlled binder-free multiwall carbon nanotubes grown on copper. 2010, ACS Nano, Vol. 4, pp. 3440-3446.

9. I. Lahiri, S.-M. Oh, J.Y. Hwang, C. Kang, H. Jeon, R. Banerjee, Y.-K. Sun, W. Choi. 2011, Ultrathin alumina coated carbon nanotubes as negative electrodes for high capacity and safe Li-ion battery. J. Mater. Chem., p. DOI: 10.1039/C1JM11474C.

10. Image processing software for microscopy, version 4.5.1. Image Metrology, DK-2970 Horsholm, Denmark.

11. I. Lahiri, D. Lahiri, S. Jin, A. Agwral, W. Choi. Carbon nanotubes: How strong is their bond with the substrate? 2011, ACS Nano, Vol. 5, pp. 780-787. 


\section{RESULTS AND DISCUSSION}

This research was aimed to develop energy-efficient devices, based on application of carbon nanotubes. Two different types of devices have been considered - field emission device and lithium-ion battery. Though these two types of devices are quite different in their operation and application, present research work offered a thread of connection between them - application of carbon nanotube as the active material in their electrodes. This chapter is the core of this dissertation, presenting detailed description and scientific analysis of the results. While sections $4.1-4.4$ describe different aspects of field emission devices, the latter sections concentrate on Li-ion battery application.

\subsection{Structure and Field Emission Performance of Conventional 2-Dimensional Field Emitters}

As has been pointed out in section 2.1.3, carbon nanotubes (CNT) are being proposed as the most suitable material for future field emission devices [1-5]. Many CNT-based field emission devices have shown excellent responses [6-10]. However, it was noted that practical field emitters, which often have a large number of vertically or randomly oriented CNTs, did not perform well up to the level of expectation, when compared to the emission response of a single emitter [11]. While it is feasible to manufacture a good junction between a single CNT emitter and its substrate (in most of the cases, the substrate is metallic), it is practically impossible to follow similar techniques to create good junctions between all emitters in a bulk field emission device with the substrate. It is also noted that many of the reported CNT based field emitters, especially for large scale applications, have been fabricated based on screen printing or 
pasting process. The interfaces between CNTs and substrates formed by these processes have shown loosely bonded structure either by van der Waals forces or weak chemical bonding. The stable CNT-substrate bonding has been in the focus for high efficiency field emission and for long term stability. Thus, apart from the performance of individual CNTs, contact between CNTs and substrate often play important role in restricting the performance of practical, bulk field emitters [11]. The so-called 'rooting' process is one of the proposed solutions to enhance CNT-substrate bonding [11, 12]. In this process, CNTs are set on a metallic foil and are allowed to be heated to high temperature $(\sim 1400$ $\mathrm{K})$ under high vacuum $\left(10^{-6} \mathrm{~Pa}\right.$ or higher) to allow sufficient diffusion and good bonding between the CNTs and the metal foil. However, this additional high temperature processing step poses several restrictions on device manufacturing. In this research work, an advanced 'rooting' technique - interface control, was presented to manipulate CNTsubstrate bonding, leading to enhanced field emission response. This technique avoids the high-temperature heat treatment step (as included in the so-called 'rooting' process [11, 12]) and allows CNT growth on a variety of substrates through thermal chemical vapor deposition (CVD) technique, which is one of the most popular synthesis routes for CNTs. As part of this process, selected metallic thin films were deposited on substrates (apart from the catalyst thin films), field emission behavior of CNTs grown on those substrates were characterized and structure and field emission behavior were correlated to finally propose efficient ways of interface-control.

In order to achieve a sustainable junction between CNTs and substrates, two issues were stressed in 'interface control' - (i) minimizing electrical resistivity and maximizing thermal conductivity at interface, and (ii) a strong bond formation between 
CNTs with the material beneath. The process involved three stages of materials selection - substrate, catalyst and an optional interface layer, which plays an extremely important role in this study.

One way to reduce interfacial resistance and enhance thermal conductivity is to adopt CNT growth on a metallic substrate having high enough electrical and thermal conductivity [13]. It may be mentioned here that CNTs can be semiconducting or metallic depending on their chiral angle and diameter, thus alignment of Fermi energy levels of $\mathrm{CNT}$ and the substrate plays a crucial role in minimizing the contact resistance. In the present study, multiwall carbon nanotubes (MWCNT) are used, which have approximately one-third of the tubes as metallic among the concentric carbon walls. In such a situation, electrical conductivity of MWCNTs are dominated by the conducting walls (with almost no role played by the non-conducting ones) and for all practical purposes, MWCNTs are considered as metallic [14]. Thus, a metallic substrate is expected to form an ohmic contact with multiwall carbon nanotubes (MWCNT). In the present study, three different metals $(\mathrm{Cu}, \mathrm{Al}$ and $\mathrm{W})$ and one ceramic, low temperature co-fired ceramic (LTCC) were selected as the substrate materials and field emission behavior of the CNTs grown on these substrates were compared with those synthesized on Si substrates. During materials selection, formation of a low-resistance ohmic contact between the CNTs and substrate material has been strongly emphasized. While $\mathrm{Cu}$ and $\mathrm{Al}$ were chosen for their excellent electrical and thermal conductivities, W was selected for its very good high temperature properties, as well as for its satisfactory electrical conductivity [15]. Si has been chosen for comparison purpose, as it has remained the most popular choice as substrate material for CNT growth in the electronics and 
semiconductor industry. LTCC, which was also used as a substrate in field emitters [16], served as another sample for comparing the effects of an insulating substrate on field emission behavior. Two well-known highly conductive metals, Ag and Au, were not considered in the present study, as these are too expensive to be considered as substrate material during CNT growth for any bulk field emission device application.

Two different catalyst layers, $\mathrm{Ni}$ and $\mathrm{Fe}$, were used in this study. It may be mentioned here that both $\mathrm{Ni}$ and $\mathrm{Fe}$ have higher electrical conductivities as compared to other commonly used catalysts, $\mathrm{Cr}$ and $\mathrm{Pd}$ (electrical conductivity of $\mathrm{Ni}, \mathrm{Fe}, \mathrm{Pd}$ and $\mathrm{Cr}$ are $0.143,0.0993,0.095$ and $0.0774,10^{6} / \mathrm{cm}-\Omega$, respectively) [15]. Samples, in the present study, used $\mathrm{Ti}$ as the main intermediate layer. Two aspects were considered during selection of the interfacial layers - electrical conductivity and ability to form strong bonds with carbon. TiN is preferred as diffusion barrier layer in many semiconductor devices, due to its good electrical conductivity [17]. However, TiN has shown tendency to be oxidized easily and hence, add extra resistance [18]. Ti, on the other hand, offers electrical conductivity similar to that of TiN [15] and is a well-known carbide former. Titanium, was thus an automatic choice in the present study. Aluminum (Al), another known carbide former metal, has much higher conductivity than Ti. In spite of its advantageous properties, Al-intermediated substrates showed poor or no CNT growth, mainly due to its low melting temperature. Thus, in conjunction with the focus of 'interface control', as depicted earlier, selection of materials in the present study was aimed to minimize interfacial resistance (to ensure an easy flow of electrons to the CNT emitters), maximize thermal conductivity and encourage strong bond formation between CNTs and substrates. Table 4.1 summarizes sample history. Some other issues like work 
function of the metal, its binding energy with carbon, type of contact etc. might also play important roles in determining the interfacial resistance and effect of those factors will be discussed in section 4.1.4.

Table 4.1: Nomenclature of the samples used in this study

\begin{tabular}{|c|c|c|c|c|}
\hline Sample & Substrate & Interface layer & Catalyst & Nomenclature \\
\hline Cu-Ti-Ni-CNT & $\mathrm{Cu}$ & $\mathrm{Ti}$ & $\mathrm{Ni}$ & $\mathrm{C} 1$ \\
\hline Cu-Ti-Fe-CNT & $\mathrm{Cu}$ & $\mathrm{Ti}$ & $\mathrm{Fe}$ & $\mathrm{C} 2$ \\
\hline Al-Ti-Ni-CNT & $\mathrm{Al}$ & $\mathrm{Ti}$ & $\mathrm{Ni}$ & $\mathrm{A} 1$ \\
\hline Al-Cu-Ti-Ni-CNT & $\mathrm{Al}$ & $\mathrm{Cu}+\mathrm{Ti}$ & $\mathrm{Ni}$ & $\mathrm{A} 2$ \\
\hline W-Ti-Ni-CNT & $\mathrm{W}$ & $\mathrm{Ti}$ & $\mathrm{Ni}$ & $\mathrm{W} 1$ \\
\hline W-Cu-Ti-Ni-CNT & $\mathrm{W}$ & $\mathrm{Cu}+\mathrm{Ti}$ & $\mathrm{Ni}$ & $\mathrm{W} 2$ \\
\hline Si-Ti-Ni-CNT & $\mathrm{Si}$ & $\mathrm{Ti}$ & $\mathrm{Ni}$ & $\mathrm{S} 1$ \\
\hline Si-Cu-Ti-Ni-CNT & $\mathrm{Si}$ & $\mathrm{Cu}+\mathrm{Ti}$ & $\mathrm{Ni}$ & $\mathrm{S} 2$ \\
\hline LTCC-Ti-Ni-CNT & $\mathrm{LTCC}$ & $\mathrm{Ti}$ & $\mathrm{Ni}$ & $\mathrm{L} 1$ \\
\hline LTCC-Cu-Ti-Ni-CNT & $\mathrm{LTCC}$ & $\mathrm{Cu}+\mathrm{Ti}$ & $\mathrm{Ni}$ & $\mathrm{L} 2$ \\
& & & & \\
\hline
\end{tabular}




\subsubsection{Structures of MWCNT Based Field Emitters}

Criteria for a good field emitter device are low turn-on field, high emission current and good stability of emission in long-time operation. Most of the researches concentrating on field emission from CNTs, have, thus, concentrated on these issues. Generally, vertically aligned CNTs are used for field emitter applications [19]. However, randomly oriented $\mathrm{CNTs}$ have also shown good field emission response $[8,20]$. In the present study, randomly oriented MWCNTs were found to be formed on all samples, during thermal CVD process. This finding is in agreement with the expectation as thermal CVD process is known to produce randomly oriented CNTs [21]. Figure 4.1 shows some representative low-magnification SEM image (and one high-magnification SEM image as inset) for some of the samples studied. It may be observed that all the samples had randomly oriented mat type structure of MWCNTs. Similar structures were observed for all other samples, too. However, a significance difference between the MWCNT structures existed in their diameter distribution. This issue will be dealt with separately in a latter sub-section. Representative Raman spectroscopic responses of the MWCNTs, grown on different samples, are presented in figure 4.2. First-order Raman spectra of MWCNTs (actually, all graphitic materials) showed a strong peak at $1580 \mathrm{~cm}^{-1}$ (' $\mathrm{G}$ ' band, which is a high frequency $\mathrm{E}_{2 \mathrm{~g}}$ first-order mode from graphite like $\mathrm{sp}^{2}$ bonds), along with an additional peak at $1350 \mathrm{~cm}^{-1}$ (' $\mathrm{D}$ ' band, which is from diamond like $\mathrm{sp}^{3}$ bonds). Since the origin of $\mathrm{D}$ band can be explained by double resonance theory, it is also indicated as $\mathrm{A}_{\mathrm{lg}} \mathrm{D}$ mode - a band caused by defects and disorder of the graphitic material. In the present study, a shift of $\mathrm{G}$ band from 1580 to $1592.4 \mathrm{~cm}^{-1}$ indicated presence of significant amount of nanocrystalline graphitic phase in the material. 

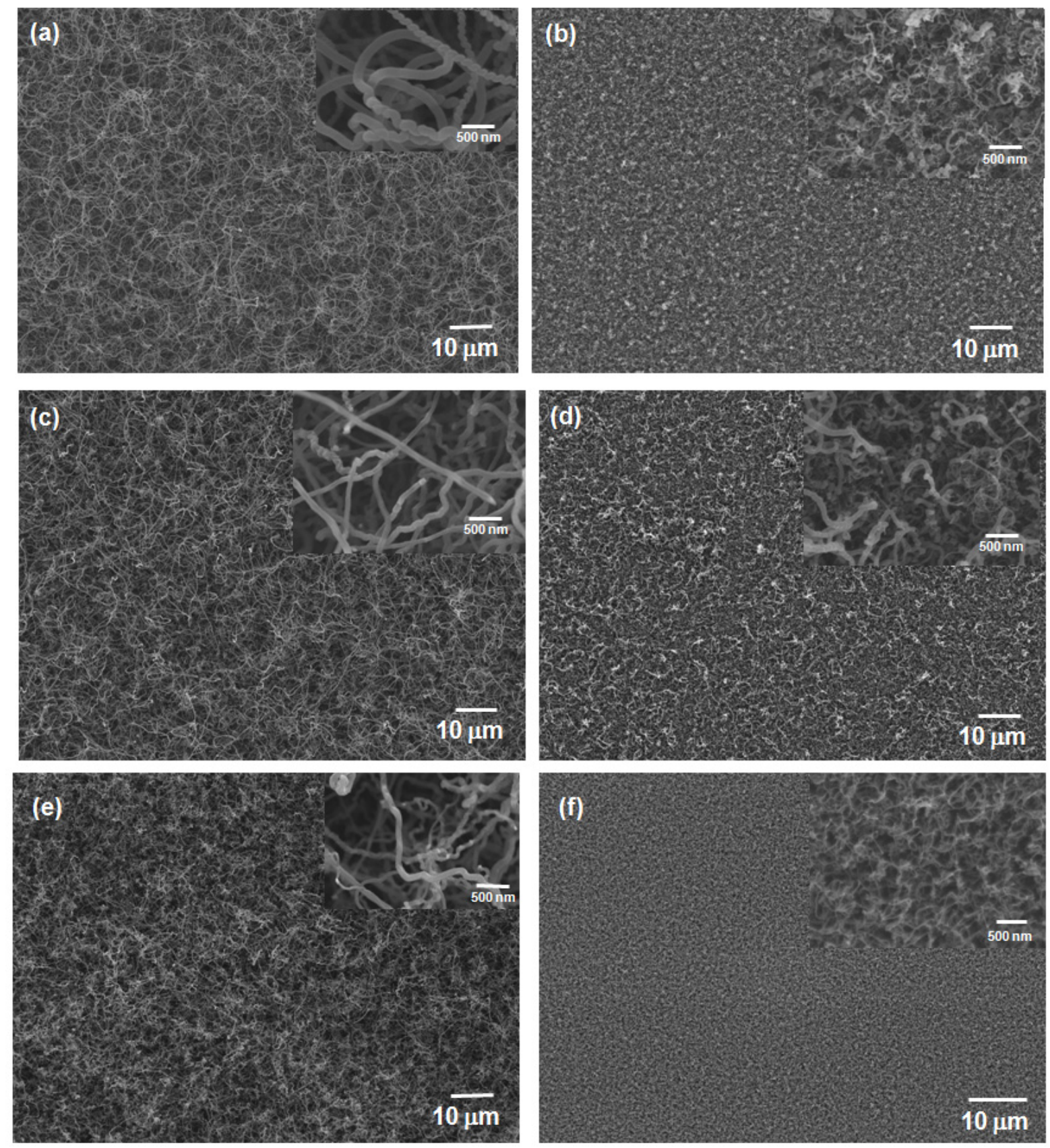

Figure 4.1: Structure of CNTs grown on Cu- and Si-based substrates. (a), (c), (e) SEM images of the MWCNTs on $\mathrm{Cu}$-Ti-Ni, $\mathrm{Cu}$-Ti-Fe and $\mathrm{Cu}$-Al-Fe samples, respectively. (b), (d), (f) SEM images of the MWCNTs on Si-Ti-Ni, Si-Ti-Fe and Si-Al-Fe samples, respectively. All the insets show high magnification images of the respective CNT structures. 


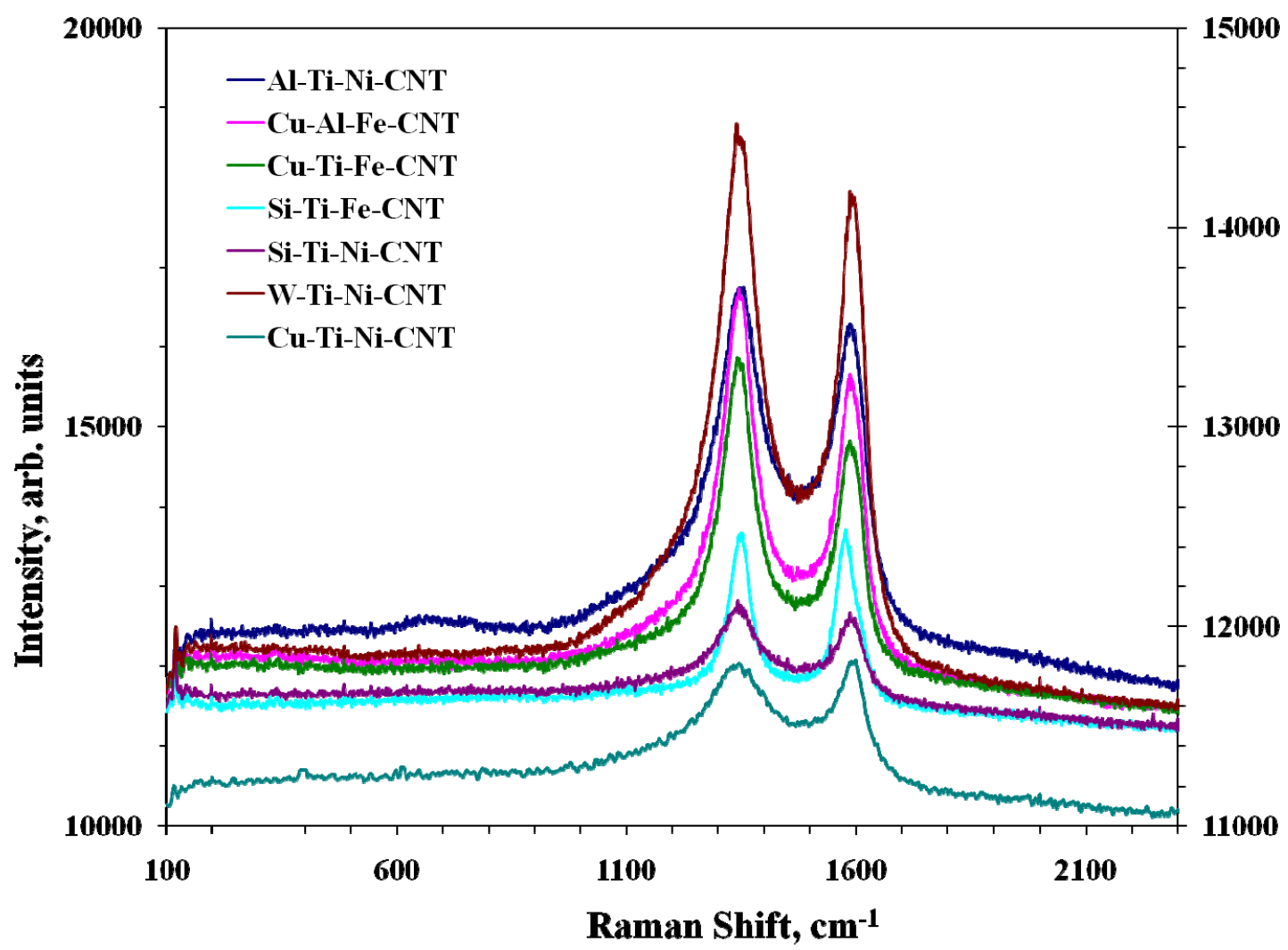

Figure 4.2: Raman spectroscopic responses of MWCNTs grown on a variety of samples.

Comparatively wider peaks of $\mathrm{D}$ and $\mathrm{G}$ bands indicate presence of disorder induced features in the graphite like $\left(\mathrm{sp}^{2}\right)$ material and predominance of tubular structures in the CNTs, respectively. One important point to be noted from this figure is that all the MWCNT structures contain high defect density (high intensity ratio of D to G peak in Raman spectra - almost equal to unity. Table 4.2 summarizes this ratio $\left(\mathrm{I}_{\mathrm{D}} / \mathrm{I}_{\mathrm{G}}\right)$ for all the samples. It may be observed from figures 4.1 and 4.2 that an overall similitude existed between all the MWCNT structures, in spite of their differences in diameter distribution. All the samples had highly dense structure of randomly oriented MWCNTs, having high defect density. This structural resemblance indicates that the CVD process was successful 
in allowing nucleation and growth of CNTs on all the substrates. However, substrateinterlayer-catalyst interaction led to different size and distribution of nano-catalytic islands, which may be held responsible for difference in diameter distribution among different samples.

Table 4.2: Raman characteristics of MWCNT emitters grown on different samples

\begin{tabular}{|c|c|}
\hline Sample ID & Raman intensity ratio $\left(\mathbf{I}_{\mathbf{D}} / \mathbf{I}_{\mathbf{G}}\right)$ \\
\hline C1 & 0.99 \\
\hline C2 & 1.07 \\
\hline A1 & 1.15 \\
\hline A2 & 0.92 \\
\hline W1 & 1.12 \\
\hline W2 & 1.14 \\
\hline S1 & 0.96 \\
\hline S2 & 1.13 \\
\hline L1 & 1.01 \\
\hline L2 & 0.96 \\
\hline
\end{tabular}


Another noteworthy feature, from figure 4.3, was absence of catalyst particles on the tip of some of the CNTs (root growth), while others showed presence of catalyst particle at the CNT tip (tip growth). Thus, the growth process, in this study, seems to be mixed-control growth of CNTs, involving a combination of root growth and tip growth.

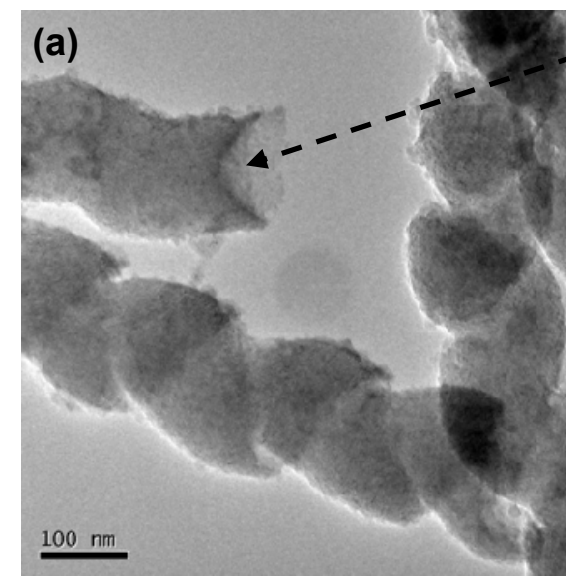

- Open tip of CNT $\mathrm{Ni}$ catalyst at

(b) tip of CNT

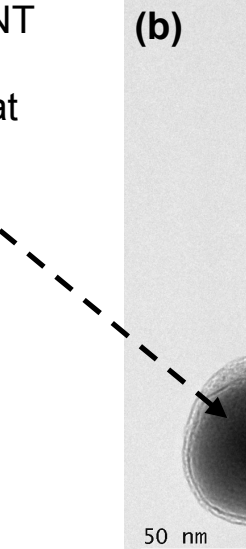

Figure 4.3: TEM images of the individual MWCNTs grown on Raman spectroscopic responses of MWCNTs grown on Si-Ti-Ni substrate, showing tips of the CNTs.

Before initiating discussion on the issues related to 'interface-engineering', it may be important to emphasize here that, except for CNT diameter, all the samples had shown similar structural features - mat of randomly oriented MWCNTs, mixed-control growth process, high defect density in CNTs. Hence, variation in the field emission response of the samples can be related to CNT diameter difference or to distinct 'interface control' of each samples.

While considering the path of an electron in a typical cathode of a field emitter device, it may be appreciated that several resistances appear in the conduit - that of 
substrate $\left(\mathrm{R}_{\text {substrate }}\right)$, interface layers $\left(\mathrm{R}_{\text {interface }}\right)$, catalysts $\left(\mathrm{R}_{\text {catalyst }}\right), \mathrm{CNTs}\left(\mathrm{R}_{\mathrm{CNT}}\right)$ and any other phase present. Total resistance $\left(\mathrm{R}_{\text {total }}\right)$ in cathode can be assumed as a summation of all these resistances. In the present study, we assumed resistance-free flow of electrons through CNTs (i.e. $\mathrm{R}_{\mathrm{CNT}} \sim 0$ ). However, it may be worth to state here that in practice CNTs may offer appreciable resistance to electron flow [22]. Though quantum resistance of CNTs is high $(\sim 12.9 \mathrm{k} \Omega)$, resistance of MWCNT bundles are often found to be quite low (30-40 $\Omega$ ), due to parallel contact of several walls of many CNTs [23]. Apart from that, several other factors such as actual fraction of semiconducting nanotubes in the structure, strain induced modification of work function [24] etc. are known to affect electron transport. In the following sections, effect of substrate materials, interfacial layers and catalysts on the field emission behavior of CNT based have been discussed.

\subsubsection{Effect of Substrate on Field Emission Response}

Field emission could be characterized either in DC or in AC bias. During application of DC bias, the emitters are expected to be damaged quickly during field emission test, owing to continuous ion bombardment. To minimize structural damage of the emitters, pulsed (AC) electric field is often applied. In the present study, field emission studies were mostly conducted under an AC applied bias. All the AC I-V plots

presented in this report consider RMS values only (unless otherwise mentioned). Field emission responses of the MWCNT structures, grown on a variety of substrates - from metallic through semiconductors to insulators, are compared in figure 4.4. All these samples used $\mathrm{Ti}$ as the interlayer and $\mathrm{Ni}$ as the catalyst. Figure 4.4 (a) shows the currentvoltage (I-V) measurement of the emitters under AC bias. It is clearly evident that CNTs 
on $\mathrm{Cu}$ substrate offered highest emission current. The turn-on fields $\left(\mathrm{E}_{\mathrm{TO}}\right.$, defined as the field required to generate $10 \mu \mathrm{A} / \mathrm{cm}^{2}$ current density) for the emitters (figure $4.4 \mathrm{~b}$ ) were found to be quite different - while $\mathrm{C} 1(\mathrm{Cu}-\mathrm{Ti}-\mathrm{Ni}-\mathrm{CNT})$ and $\mathrm{W} 1$ (W-Ti-Ni-CNT) showed low values $(<1.0 \mathrm{~V} / \mu \mathrm{m})$, other samples illustrated quite high $\mathrm{E}_{\mathrm{TO}}$ values $(\sim 2.0 \mathrm{~V} / \mu \mathrm{m}$ or more).
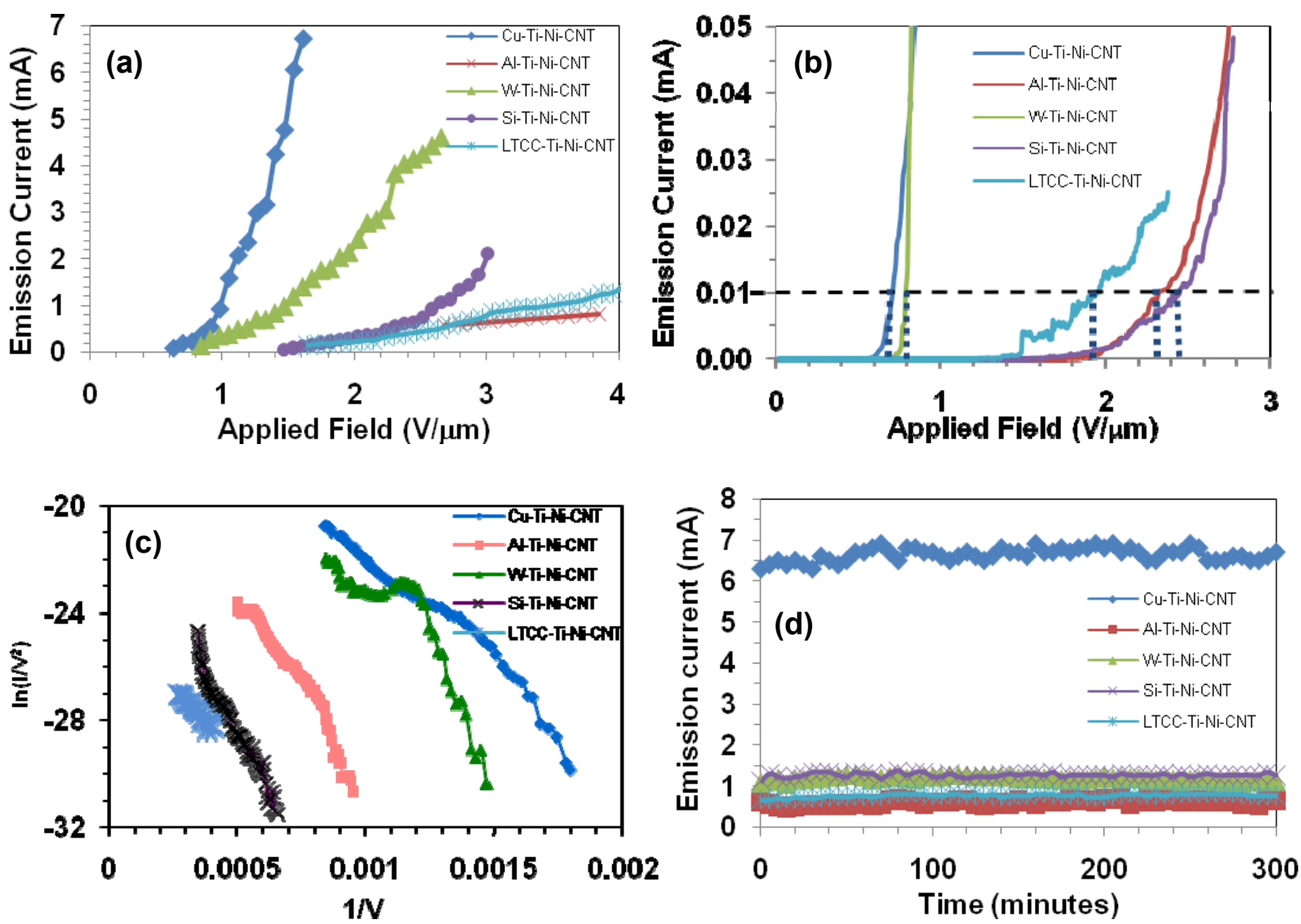

Figure 4.4: Field emission response of MWCNT emitters on Ti-intermediated substrates.

(a) Field emission current, (b) Turn-on fields (shown by the dotted lines), (c) FowlerNordheim plots and (d) long-time emission stability of Cu-Ti-Ni-CNT, Al-Ti-Ni-CNT, WTi-Ni-CNT, Si-Ti-Ni-CNT and LTCC-Ti-Ni-CNT samples. 
Straight line behavior of the conventional Fowler-Nordheim (F-N) plots (figure 4.4 c) of all the emitters, except W1 (W-Ti-Ni-CNT), confirmed the emission process to be tunneling of electrons across the energy barrier. Appearance of a knee in W-substrate sample may be related to two possible causes. First, appearance of non-linearity (or, to be more specific, a 'knee' type shape) in the F-N plot could be related to either presence of other emission processes [25] or an extra energy barrier to the flow of electrons [26]. Second, it is known that at higher current levels, the emitter structure slowly degrades and the deteriorating CNT-substrate electrical contact adds an extra series resistance, which leads to a voltage drop and saturation of emission current. In such circumstances, the field emission response of the emitter deviates considerably from the conventional Fowler-Nordheim behavior [27]. It may be observed that for the W1 sample also, the deviation from F-N behavior was found at higher current levels. Thus, it seems to be feasible that the emitter structures in this sample (W1) degraded at high emission current levels. Apart from this possible degradation of the emitter structure at higher current levels, W1 sample had shown comparable $\mathrm{E}_{\mathrm{TO}}$ and emission current with that of $\mathrm{C} 1(\mathrm{Cu}-$ Ti-Ni-CNT). Another metallic (Al) substrate based emitter A1 (Al-Ti-Ni-CNT), however, had shown poor field emission responses, probably due to its low melting temperature (which is very close to the CVD temperature followed in the present study). Compared to $\mathrm{Cu}$ and $\mathrm{W}$, both $\mathrm{Si}$ and LTCC samples have presented higher $\mathrm{E}_{\mathrm{TO}}$ and lower emission current. It may be noted here that highly conductive metallic substrates are expected to offer less resistance to electron flow and thus, minimize the contribution of $R_{\text {substrate }}$ to $\mathrm{R}_{\text {total. }}$ 
Practical application of an emitter necessitates stability of the emission current during high-current, long-time exposure. To examine the emission stability of the CNTbased emitters, stability test was performed on each of them, in a diode set-up, applying a fixed $\mathrm{AC}$ bias to it and measuring the emission current as a function of time. The results are included in figure 4.4 (d). It may be observed that all the emitters showed good stability during its continuous 5-hour long exposure, but at a different current level. C1 (Cu-Ti-Ni-CNT) had again offered best performance among all the samples considered and was stable at an emission current level of $\sim 6.4 \mathrm{~mA}$. All other samples offered stable emission at a current level of $1-2 \mathrm{~mA}$. Thus, it seems the CNT emitters formed on $\mathrm{Cu}$ substrate were robust enough against possible structural degradation. An overall better performance of $\mathrm{C} 1$ sample ( $\mathrm{Cu}-\mathrm{Ti}-\mathrm{Ni}-\mathrm{CNT})$ compared to rest four types of samples could be related to several favorable properties of $\mathrm{Cu}$. First, high electrical conductivity of $\mathrm{Cu}$ (highest among the substrates under test) ensured minimum $\mathrm{R}_{\text {substrate }}$ and allowed more current to be passed through it. Second, high thermal conductivity of $\mathrm{Cu}$ also ensured efficient heat transfer from the CNT-substrate interface and improved structural stability of the emitters. Third, CNT formed strong bonding with Ti-intermediated $\mathrm{Cu}$ substrate, which might be due to formation of a local intermetallic between $\mathrm{Cu}$ and Ti. Moreover, $\mathrm{Ti}$ also forms $\mathrm{TiC}$ and strengthens the bonding across $\mathrm{Cu}-\mathrm{Ti}$-CNT. Figure 4.5 presents TEM image taken from the interface to show good bonding between substrate and CNTs. Presence of TiC at the interface enhanced bonding between CNTs and substrate. A strong interface between CNTs and the substrate contributed to structural stability and lower interfacial resistance, leading to enhanced field emission response. 

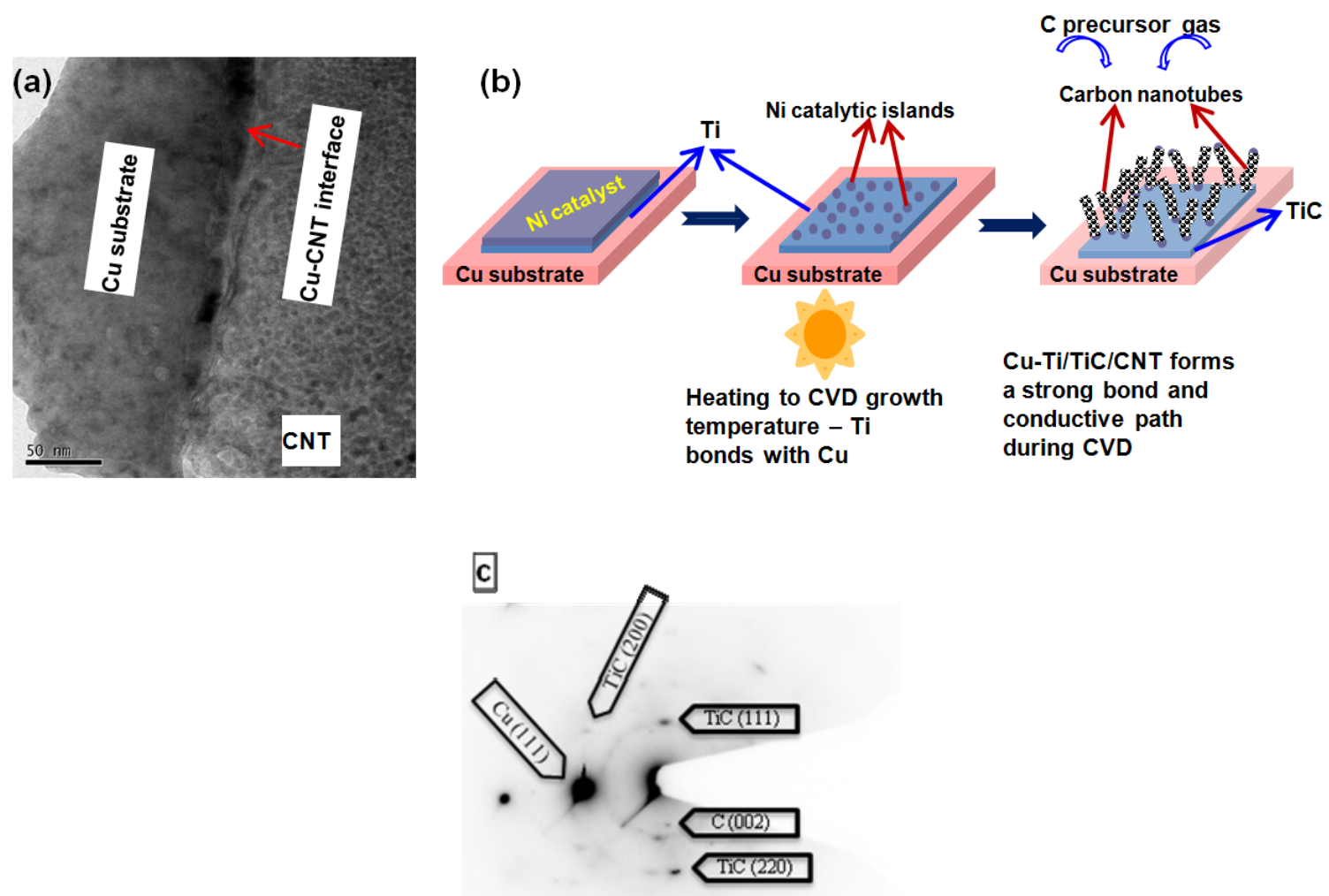

Figure 4.5: (a) TEM image of the interface between CNTs and Cu. (b) A schematic of the process showing strong bonding and conductive path formation during CVD growth of CNTs, which leads to enhanced performance of CNTs-on-Cu field emitter. (c) Diffraction spots of TiC in the selective area diffraction pattern, taken from the interface.

\subsubsection{Effect of Interfacial Layer on Field Emission Behavior}

In this study, $\mathrm{Ti}$ was selected as the interface layer, because of its high conductivity and carbide formation ability. For comparison purpose, Al was also used as interface layer, but all the samples have shown no or negligible CNT formation. In spite of $\mathrm{Al}$ having higher conductivity than $\mathrm{Ti}$, this behavior can probably be related to higher 
growth temperature $(\sim 923 \mathrm{~K})$ used during CVD process. It seems at that high temperature, which was very close to the melting point of pure $\mathrm{Al}$, the $\mathrm{Al}$ underlayer became semi-solid and behaved in a different manner and failed to form catalytic nanoislands for proper CNT growth. It may be mentioned here that further experiments (probably an in-situ SEM characterization would be best) are required to be performed to confirm this presumption regarding the nature of $\mathrm{Al}$ underlayer at the CNT growth temperature.

To highlight the importance of the interfacial layers, an additional layer of ultrathin $(\sim 15 \mathrm{~nm}) \mathrm{Cu}$ was deposited beneath $\mathrm{Ti}$, in all the samples except the $\mathrm{Cu}$-substrate based ones. Comparison of field emission response of these sets of samples is presented in figure 4.6 (next page).

It may be noted that all the samples have shown appreciable improvement in emission current and stability, when the samples had the extra $\mathrm{Cu}$-interface layer. The observation leads to a conclusion that irrespective of the nature of the substrate material (i.e., metallic, semiconductor or insulator), inclusion of the ultra-thin $\mathrm{Cu}$ layer on the substrate aids to improve the field emission response from the CNT emitters, directly grown on these substrates. Comparing with our previous experience (figure 4.4), which showed a clear level of difference in field emission behavior of CNT emitters grown on a variety of substrates, the present observation highlights the possibility of manufacturing CNT-based emitters on any kind of substrate by introduction of $\mathrm{Cu}$ intermediate layers, without much affecting their emission responses. 

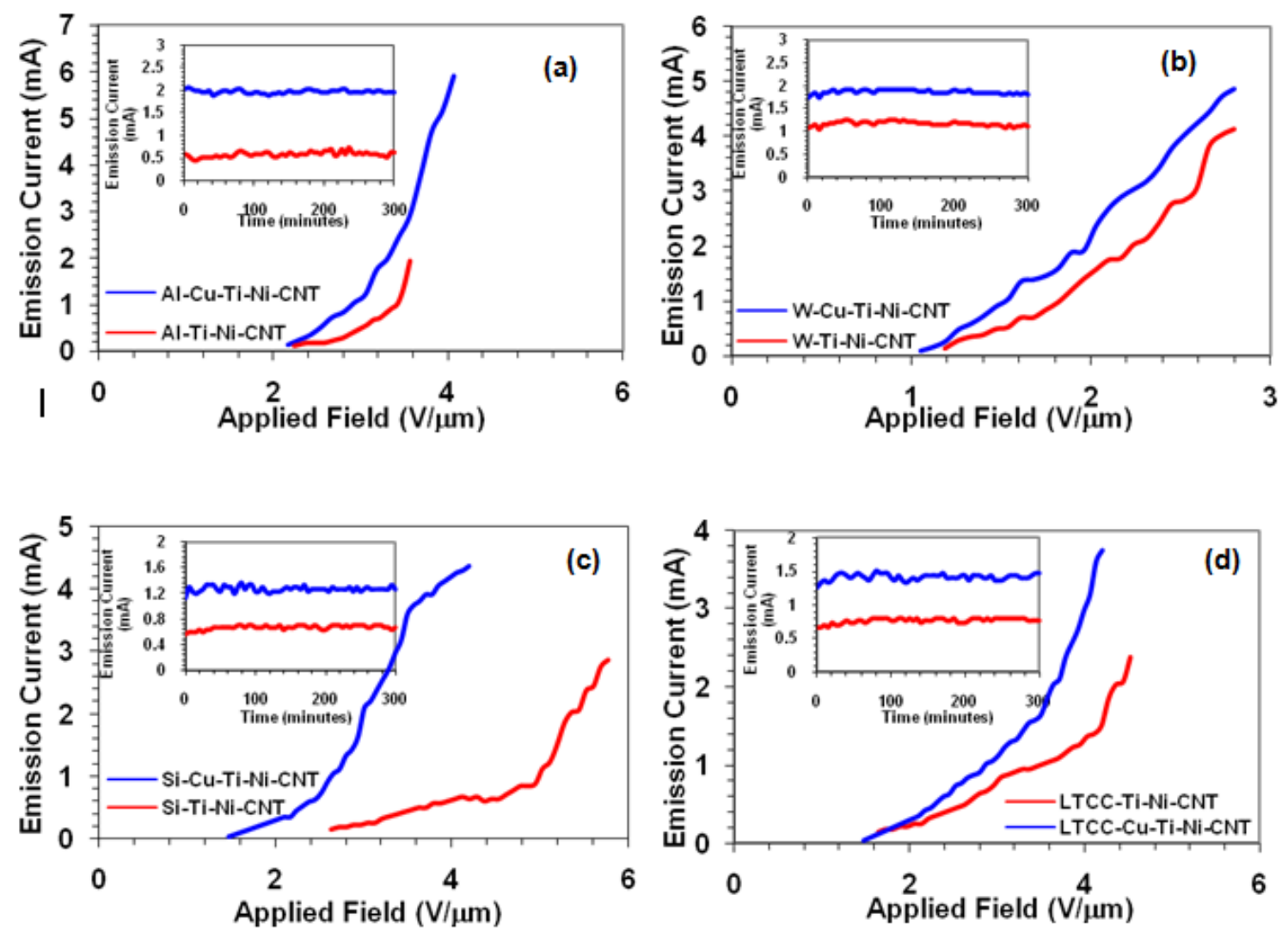

Figure 4.6: Field emission responses of MWCNT emitters on Ti-intermediated and Cu-

Ti-intermediated substrates. Field emission current and emission stability (insets) of CNT emitters on (a) Al-, (b) W-, (c) Si- and (d) LTCC-substrates.

Enhancement in field emission behavior may be related to the structures of the CNTs, grown on these substrates. As has been mentioned previously, similar structural features - mat of randomly oriented MWCNTs, mixed-control growth process, high defect density in CNTs, were observed for all the samples. So, it seems either CNT diameter difference or 'interface control' of each sample could be related to this variation in field emission response. It may be recalled here that the field enhancement factor $(\beta)$ in Fowler-Nordheim equation is inversely proportional to radius of the emitter [28, 29]. Recently, it has also been shown that the contact resistance has a diameter dependant 
component, which is inversely related to the resistance term [30]. Thus, it is expected that thinner CNTs will have higher contact resistance leading to reduced emission response. On the other hand, thinner CNTs will also have reduced tip diameter, which improves field enhancement factor. Thus, two opposing effects of CNT diameter are going to compensate each other.

To realize importance of this issue in the present case, CNT diameters (for all the samples) were measured (diameters of more than $200 \mathrm{CNTs}$, randomly selected from different regions of each sample, were measured) and are plotted in figure 4.7, in the form of a statistical 'box plot'. The plot shows that the CNT diameter distribution is spread over a wide range. A closer comparison between the diameter distributions, however, did not show any kind of direct relation between CNT diameter and their field emission response.

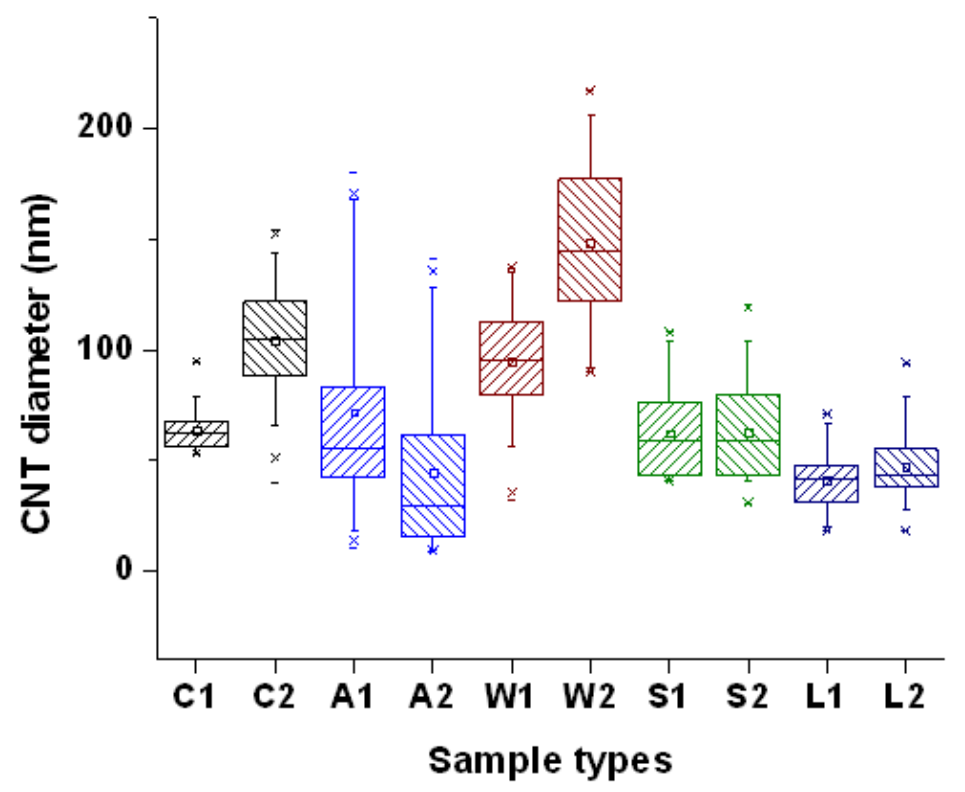

Figure 4.7: Statistical box plot showing CNT diameter distribution of all the samples considered in the present study. Refer to table-1 for nomenclatures of the samples. 
Introduction of ultra-thin $\mathrm{Cu}$ layer on each of these metallic, semiconductor and insulating substrates are expected to enhance field emission response of CNTs grown on them due to several factors. One of the important steps of field emission process is electron injection into the emitter [31] (which is CNT, here). A low-resistance, allmetallic (or, in other word, ohmic) contact between substrate-CNT ensures minimization of interfacial resistance and efficient flow of electrons from the substrate to CNTs. MWCNTs being predominantly metallic, ohmic contacts are formed with metallic substrates. However, other materials present in the interface also plays important role in deciding the contact resistance. Ti was present in all the samples as the interfacial layer. LTCC anyway forms a high resistance interface with $\mathrm{Ti}$, as it is an insulator. The p-type (100) $\mathrm{Si}$, used in the present study, formed a schottky barrier with $\mathrm{Ti}$, due to fermi energy alignment between $\mathrm{Si}$ and $\mathrm{Ti}[32]$. Though $\mathrm{W}-\mathrm{Ti}$ and $\mathrm{Al}-\mathrm{Ti}$ interfaces formed ohmic contacts, their interface resistances were expected to be higher. This could be explained by the tendency of $\mathrm{W}$ and Ti to remain as separate phases at the CVD temperature, while Al prefers to form solid solution [33]. It may be noted here that intermetallics show significantly lower resistance, as compared to solid solutions or mixture of two phases [34]. Hence, formation of an intermetallic, at the interface, is expected to offer two benefits - better conductivity and stronger bonding. Introduction of $\mathrm{Cu}$ at the interface, in all these samples, favored formation of $\mathrm{Cu}-\mathrm{Ti}$ intermetallic phase at the interface [35]. Thus, at a given bias, electrons could move much easily from the substrate to the CNTs, through the $\mathrm{Cu}$-intermediated samples. Moreover, reduction in interfacial resistance also decreased the total energy barrier in the electron path and thus, enhanced electron tunneling from the CNTs. Further $\mathrm{Cu}$, being metallic, also acted as a source of electrons, 
which ensured availability of more electrons during the emission process. $\mathrm{Cu}$ also acted as a heat sink during the emission process, which efficiently removed heat generated during electron emission and thus, minimized degradation of CNT emitters during longtime exposure. $\mathrm{Cu}-\mathrm{Ti}-\mathrm{Ni}$ combination also offered a strong bonding with the CNTs (will be discussed in detail in section 4.2), which improved the structural stability of the emitter and hence, the emission stability. Considering all these facts, it seems obvious that introduction of an ultra-thin layer of $\mathrm{Cu}$, on any kind of substrate, aids in enhancing the field emission response of CNTs, directly grown on them. This finding widens the application regime of CNT based field emitters on a variety of substrate materials, from metals to insulators, without severely compromising with their emission responses. It also proves the importance of 'interface-control' in determining the field emission response.

\subsubsection{Effect of Catalyst Layer on Field Emission}

After analyzing the contribution of substrate and interface layers in controlling the field emission behavior of CNT emitters, through the so-called 'interface engineering', the next emphasis was on the effect of catalyst on field emitter performance. The present study followed thin film catalyst deposition by sputtering. During CVD, the catalyst formed a homogeneous distribution of nano-islands, on which CNTs were synthesized. It is well-known that the catalytic island formation initiates by dewetting from the substrate surface and final shape, size and crystallinity of these islands depend strongly on the minimization of surface and interface energy [36]. CNT diameters closely relate to the size of the catalyst islands. Thus, catalyst selection, its thickness and its interaction (specifically wetting) with the material beneath it, plays important role. To highlight the 
effect of catalyst material, thickness of catalyst was maintained at a constant level $(\sim 10$ nm) throughout this study.

Findings described in the previous sub-sections showed that $\mathrm{Cu}$ - $\mathrm{Ti}$ combination worked very well, as long as field emission behavior of CNTs grown on them was concerned. Hence, this combination was further used during the tests to understand the effect of catalysts. Apart from Ni, which was used for all the samples discussed so far, $\mathrm{Fe}$ was used as catalyst for another set of samples, having $\mathrm{Cu}$ as substrate and Ti as interface layer. Field emission response of this sample $(\mathrm{C} 2, \mathrm{Cu}-\mathrm{Ti}-\mathrm{Fe}-\mathrm{CNT})$ is compared against $\mathrm{C} 1(\mathrm{Cu}-\mathrm{Ti}-\mathrm{Ni}-\mathrm{CNT})$ in figure 4.8 .
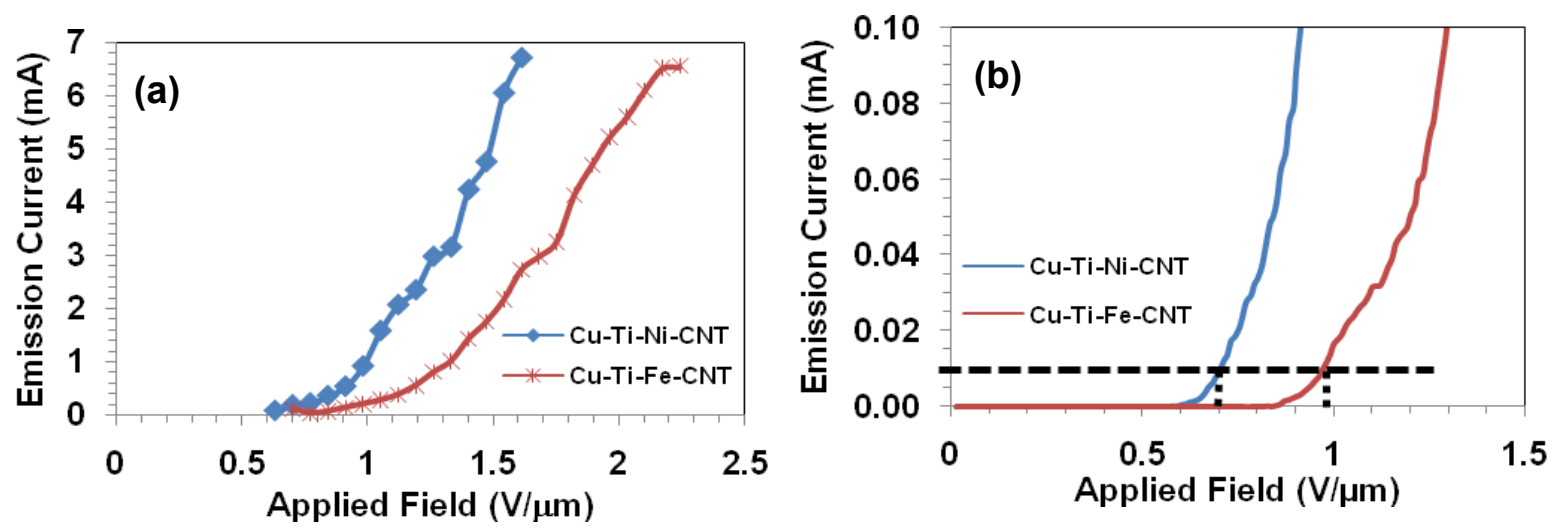

Figure 4.8: Effect of catalyst ( $\mathrm{Ni}$ or $\mathrm{Fe}$ ) on field emission response of CNTs directly grown on Cu substrates. (a) Field emission current and (b) turn-on field for Cu-Ti-NiCNT and $\mathrm{Cu}$-Ti-Fe-CNT samples.

It shows that replacing $\mathrm{Ni}$ by $\mathrm{Fe}$ as catalyst causes a up shift in $\mathrm{E}_{\mathrm{TO}}$ from 0.7 $\mathrm{V} / \mu \mathrm{m}$ to $1.0 \mathrm{~V} / \mu \mathrm{m}$ and an increase in the field required to produce comparable emission current ( $7 \mathrm{~mA})$. A close look into CNT diameter distribution in figure 4.7 indicates that 
the difference in field emission response of these two samples could be related to diameter effect. Moreover, higher resistance of $\mathrm{Fe}$, as compared to $\mathrm{Ni}$ (electrical conductivity of $\mathrm{Ni}$ and $\mathrm{Fe}$ are 0.143 and $0.0993,10^{6} / \mathrm{cm}-\Omega$, respectively [15]) is also expected to contribute more towards $\mathrm{R}_{\text {catalyst }}$ and thus, to the total resistance, $\mathrm{R}_{\text {total. }}$. Further studies are required to conclusively determine which factor affects field emission behavior most.

\subsubsection{Other Possible Factors Affecting Field Emission}

In the previous sections, it was shown that 'interface engineering' through substrate optimization, inclusion of a suitable interface layer, choice of catalyst material played important role in determining the emission properties of CNTs, grown onto them. Appreciable emission could even be obtained from CNT emitters, grown on an insulator substrate, when an ultra-thin conductive copper layer was introduced onto it. Studies, reported so far, have also not found any conclusive relation between CNT diameter and field emission behavior. In this section, an effort will be made to relate field emission properties with other possible factors.

Conduction property and work function of the metal contact has been known to affect CNT-field effect transistor (FET) properties, but mainly for single-wall carbon nanotubes (SWCNT) [37-39]. However, in such studies, it was found that Ti formed much stronger bonding with CNTs [37, 39] and both Ti and Ni formed low-resistance ohmic contacts with various semiconducting and metallic SWCNTs [39, 40]. Though these studies were performed for SWCNTs, still these observations give us an idea about suitability of Ti and Ni, in contact with CNTs. At par with this theoretical expectation, 
results of the present study revealed that, out of the different varieties of underlayercatalyst combinations used, Ti-Ni combination outperformed all others.

Apart from these issues, type of contact also affects the interfacial resistance for MWCNTs [41, 42]. For side-contacted CNTs, area of contact directly impact the electron transmission process by imposing a finite energy barrier created by van der waals interaction between metal and CNTs. This interaction was found to be significant for smaller contact areas; though for larger contact areas, better electronic coupling between metal and MWCNTs are reported [42]. Further, contacting multiple walls of concentric nanotubes, which is a definite possibility in the present study, is known to reduce interface resistance [42]. Thus, contact between MWCNTs and the material underneath were not expected to create any extra resistance since the CNTs were synthesized directly onto substrate via chemical bond formation, in the present study.

After explaining the importance of catalyst and barrier layer materials, it is important to understand the effect of the substrate. This observation may also be explained in terms of properties of substrate materials. Apart from offering high electrical (aiding faster electron transport) and thermal conductivity (effectively dissipating heat generated during emission process and thus, minimizing de-bonding of CNTs from substrate), metallic substrates form ohmic contacts with MWCNTs, thus creating a low substrate-CNT barrier. Hence, it is expected that electrons from CNTs grown on metallic substrates will be able to tunnel through the energy barrier at a much lower excitation field, thus showing lower $\mathrm{E}_{\mathrm{TO}}$ values. Among the metallic substrates, the material with higher work function $(\varphi)$ (but nearer to that of CNTs) need least energy to inject electrons into CNTs, especially metal contact with semiconducting CNTs and hence, show 
favorable F-N tunneling [42]. However, for metals with lower work function than that of CNTs, the junction will behave as an ohmic one. In light of this observation, it is evident that $\mathrm{Cu}(\varphi=4.65 \mathrm{eV}), \mathrm{W}(\varphi=4.55 \mathrm{eV})$ and $\mathrm{Al}(\varphi=4.28 \mathrm{eV})$ will form low-resistance ohmic contact with CNTs (assuming its work function to be $5 \mathrm{eV}$ ). On the other hand, $\mathrm{Si}$, being a semiconductor, the nature of the interface depends strongly on Fermi energy level alignment of $\mathrm{Si}$ and the interfacial materials. In the present study, a p-type (100) $\mathrm{Si}$ is used, which has work function value of $4.91 \mathrm{eV}$, while Ti has much lower work functions $(4.33 \mathrm{eV})$ [32]. Thus, schottky junction is formed at the Si-Ti interface. Schottky junction offers much higher interfacial resistance and hence, delays the initiation of field emission process. However, in spite of having higher conductivity, Al-substrate based CNT emitter does not perform well during field emission which could be related to higher CVD temperature, as mentioned earlier. Moreover, solid solution formation tendency of the AlTi may also be responsible for its poor field emission response. This issue is explained further in next paragraph.

Another important factor for a good field emitter is bonding between CNTs with the materials underneath. It has been reported that poor adherence between substrateCNT influence field emission by adding an extra resistance and often leading to failure of the device [43]. Good bonding results in lowering of contact resistance and a high, sustained emission current $[44,45]$. Ti, as already been explained previously, forms strong bond with CNTs $[37,39]$. Moreover, $\mathrm{Cu}$ reacts with Ti above 873K (CNT growth temperature, used in the present study, was higher than $873 \mathrm{~K}$ ) to form $\mathrm{Cu}-\mathrm{Ti}$ intermetallic phase [35] and this intermetallic formation is expected to enhance adhesion between them. Further, presence of an intermetallic is known to enhance electrical 
conductivity of the material, as compared to presence of mixture of two elements or even a solid solution [34]. While $\mathrm{Cu}-\mathrm{Ti}$ is known to form intermetallic easily, all other systems, considered during the present course of study, either do not form intermetallic (Si-Ti, Si-Al, W-Ti) or prefer to form solid solutions (Cu-Al, Al-Ti) [33]. Thus, considering all the relevant materials' properties, selection of $\mathrm{Cu}$, Ti and $\mathrm{Ni}$ as substrate, barrier layer and catalyst, respectively, is expected to lead to an efficient CNT growth and good field emission from them. The results obtained in the present study fully support this observation.

A variation in the nature of CNTs, grown on different substrates, could possibly be another reason for different field emission behavior. Under an applied external electric field, the triangular barrier between the Fermi level of the bottom contact and the conduction band of CNTs narrows down, facilitating efficient electron tunneling to the CNTs. It is known that while $\mathrm{sp}^{2} \mathrm{C}$ cluster offers conducting channels, thus improving electron transfer from the substrate to the emitter tip [46], $\mathrm{sp}^{3}$ nature of the carbon present in the structure reduces the electron affinity and hence, shrinks the potential barrier supporting easy escape of electrons into vacuum [47]. Moreover, $\mathrm{sp}^{3}$ type of defects in an otherwise $\mathrm{sp}^{2}$ network of graphitic materials is also known to lower local work function [48]. In the present study, Raman spectra of the CNTs, grown on all the substrates, have shown presence of both $\mathrm{sp}^{2}$ and $\mathrm{sp}^{3}$ bonds in the MWCNT structures. Thus, from the point of view of contribution of $\mathrm{sp}^{2}$ and $\mathrm{sp}^{3}$ bonds to field emission, all samples were almost similar. So, variation in nature of CNTs, grown on different substrates during this study, also can not be related to their diverse field emission behavior. 


\subsubsection{Importance of Interface Engineering on Field Emission}

In the present study, different material combinations were used as substrate $(\mathrm{Cu}$, $\mathrm{Al}$, W, Si and LTCC), interface layer ( $\mathrm{Ti}$ and $\mathrm{Cu}$ ) and catalyst $(\mathrm{Ni}$ and $\mathrm{Fe}$ ) for MWCNT growth with an aim to optimize their field emission response. 'Interface engineering', through application of suitable substrate, interface layer or catalyst, has been shown to play an extremely important role in maximizing the field emission responses. Analysis of the structural and field emission data proposes $\mathrm{Cu}$, Ti and $\mathrm{Ni}$ to be the best combinations for substrate, underlayer and catalyst, respectively, among the materials studied. Figure 4.9 compares literature reported field emission data with those of the best sample (Cu-TiNi-CNT) in the present study. Two important parameters of field emission, turn-on field and field enhancement factor $(\beta)$, were compared.

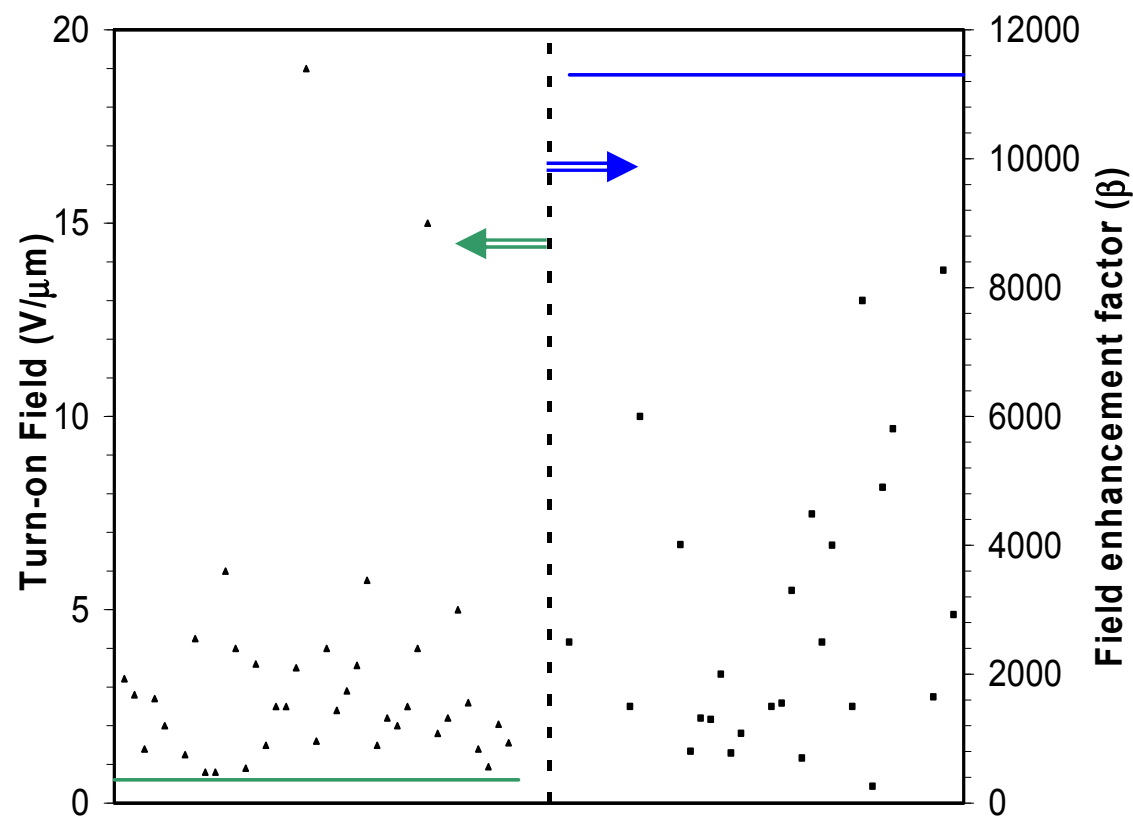

Figure 4.9: A comparison of the achievements of the present study with the literature reported values, for turn-on field (left half) and field enhancement factors (right half) for 
CNT based field emitters. While the points denote literature values [13, 21, 49-73], the lines represent the status of the present study.

The figure clearly shows the superiority of the MWCNT field emitter structure, produced in the present study, with the lowest turn-on field and highest $\beta$. However, few reports showed almost comparable turn-on field $[53,54]$ or high total emission current [44]. In all those cases, CNT field emitters were prepared using special techniques, including sandwitch-growth technology, micro or nano patterning, microwave plasma enhanced CVD etc., to grow well-patterned and aligned CNTs. The present study used a simple thermal CVD processing technique to grow randomly oriented CNTs, showing a very high field emission application potential. An intelligent choice of substrate, barrier layer and catalyst has probably contributed most to the excellent contact degradation resistance of the structure.

Introduction of an extra $\mathrm{Cu}$ interface layer was found to enhance field emission properties of CNT emitters grown on any kind of substrate. Significance of 'interface engineering' has been explained in terms of minimizing total resistance, better thermal conductivity, proper work function etc. The study highlighted the importance of 'interface engineering' in order to be able to predict the optimized interface for development of an efficient CNT-based field emitter. In future, large-scale experiments should be performed to cover all possible materials and to know the best possible materials for an optimized field emission performance of MWCNT-based field emitters.

Another important issue of scientific interest would be the emission sites of this emitter structure. In spite of the concern that the actual emission sites of these randomly 
oriented CNTs could be different from CNT tips, sufficient proof is not available. Published reports [74-76] have not pointed out to any emission site, other than the CNT tips. High $\beta$ values found in our study also indicate that the emission site is actually very fine and probably the CNT tip. However, this is an indirect support and a detailed study involving simulation of the emitter system or in situ field emission test within SEM [77] may throw some light on this issue.

\subsection{Substrate-CNT Bonding Strength}

Section 4.1 has demonstrated experimental results and analysis to prove importance of interface engineering for field emission devices. As a matter of fact, substrate-nano material (be it CNT or any other nano material) bonding is one of the fundamental issues for any type of nano devices. A poor nanomaterial-substrate adhesion may cause unreliable behavior and a very short life span of such a device. Hence, knowledge about nature and strength of the nanostructure-substrate bond is considered as one of the most fundamental issues. It is of utmost importance to device a methodology to quantify the nanomaterial-substrate bonding, to apply that technique to understand bonding between different nanostructure-substrate bonding, ultimately leading to development of well-bonded nanostructured devices. CNTs, being the focus material in the present research work, were used to quantify this bonding energy.

Many researchers have already made efforts to understand and explain this critical issue of CNT-substrate adhesion or bonding. Table 4.3 summarizes the main efforts undertaken till the planning period of the present study. The qualitative methods do not offer any standardized procedure and reproducible results, as the techniques are user 
sensitive. The quantitative methods, experimented so far, have some common ground for comparison of adhesion strengths, though all these techniques pose several limitations. First, none of these methods have been calibrated to single out the bonding between only CNT-substrate. These methods do not show the amount of stress required to pull out the adhesive tape from a blank substrate (without having CNTs grown on it) [78-80]. Thus, the strength values found in these methods are often overestimated values of exact contribution from CNT-substrate bond. Second, the adhesion strength was calculated assuming the area of the tape as the contact area. The actual contact area, where CNTs are in contact with the tape, is much lower. So, the reported values are likely to be an underestimate of the actual strength required to de-bond CNTs from the substrate. Third, the number of CNTs in contact with the tape was not known and hence, none of these methods could predict adhesion strength of a single CNT. Furthermore, it was not known whether the CNTs were breaking at the CNT-substrate interface or at any defective locations along its length or if one or few layers of multiwall CNTs were being detached or whether the CNTs were detaching from the adhesive tape itself and not removed from the substrate. These uncertainties of the reported methods lead to unknown errors in the evaluation of bond strength. Therefore, it is essential to design a new test methodology, which can more reliably characterize the bond strength between CNTs and their substrates. Since the area of importance for CNT-substrate adhesion is the interface (at nano level), a nano-level characterization technique should be best suitable for this purpose. Keeping this point in mind, during the present study, a new methodology of nano-scratch technique was developed to quantify CNT-substrate bonding, overcoming limitations of the existing techniques. 
Table 4.3: Overview of literature reported CNT-substrate adhesion testing methods

\begin{tabular}{|c|c|c|c|}
\hline Techniques & $\begin{array}{l}\text { Adhesion strength } \\
\text { or energy }\end{array}$ & Remarks & Reference \\
\hline $\begin{array}{l}\text { Blowing, rubbing, } \\
\text { brushing }\end{array}$ & NA & Qualitative method, unreliable technique, extremely user sensitive & [81] \\
\hline $\begin{array}{l}\text { Ultrasonication in } \\
\text { solvent }\end{array}$ & NA & $\begin{array}{l}\text { Qualitative method, widely used for understanding bond of CNTs } \\
\text { with substrates, results may vary in different laboratories }\end{array}$ & {$[82-86]$} \\
\hline $\begin{array}{l}\text { Dropping, shaking, } \\
\text { bending samples }\end{array}$ & NA & Qualitative method, unreliable technique, extremely user sensitive & [87] \\
\hline $\begin{array}{l}\text { Peel test using adhesive } \\
\text { tape }\end{array}$ & NA & $\begin{array}{l}\text { Qualitative method, widely used for understanding bond of CNTs } \\
\text { with substrates, highly operator sensitive }\end{array}$ & [87-89] \\
\hline $\begin{array}{l}\text { Pulling CNTs by } \\
\text { tweezers }\end{array}$ & NA & $\begin{array}{l}\text { Qualitative method, result may vary for different operators or } \\
\text { tweezers }\end{array}$ & {$[90]$} \\
\hline $\begin{array}{l}\text { Hanging known } \\
\text { weights from substrate }\end{array}$ & $0.12-0.18 \mathrm{MPa}$ & $\begin{array}{l}\text { Used for quantifying strength of CNT based adhesive tapes: } \\
\text { Able to quantify adhesion of CNTs with substrate but not } \\
\text { calibrated against standards, } \\
>\text { Only a range of de-bonding stress could be predicted in } \\
\text { absence of continuous load-displacement plot, }\end{array}$ & {$[91,92]$} \\
\hline
\end{tabular}




\begin{tabular}{|c|c|c|c|}
\hline & & $\begin{array}{l}\text { Stress can not be predicted as contact area is not known, } \\
\text { Accurate breaking/de-bonding position (whether at CNT- } \\
\text { substrate interface or along the length of CNT itself) is not } \\
\text { known } \\
>\text { Can not predict bond strength/energy for single CNTs }\end{array}$ & \\
\hline $\begin{array}{l}\text { Tensile test after } \\
\text { wrapping the CNTs by } \\
\text { adhesive tape }\end{array}$ & $0.26-0.50 \mathrm{MPa}$ & $\begin{array}{l}\text { Used for quantifying bonding of CNTs grown on wire Not } \\
\text { calibrated against standards, } \\
>\text { Stress can not be predicted as contact area is not known, } \\
>\text { Accurate breaking/de-bonding position not known } \\
>\text { Can not predict bond strength/energy for single CNTs }\end{array}$ & {$[93,94]$} \\
\hline $\begin{array}{l}\text { Compression test using } \\
\mathrm{Cu} \text { tape to contact } \\
\text { CNTs }\end{array}$ & $2.05 \mathrm{MPa}$ & $\begin{array}{l}\text { Used for quantifying bonding of CNTs grown on flat } \\
\text { substrates: } \\
>\text { Not calibrated against standards, } \\
>\text { Stress can not be predicted as contact area is not known, } \\
>\text { Accurate breaking/de-bonding position not known } \\
>\text { Can not predict bond strength/energy for single CNTs }\end{array}$ & [95] \\
\hline
\end{tabular}




\subsubsection{Bonding Energy Quantification by Nano-Scratch}

Nano-scratch technique is well established for measurement of mechanical properties in two-dimensional nanomaterials like thin films used in magnetic storage materials, micro-electromechanical systems (MEMS) etc. [96, 97]. However, application of this technique to quantify CNT-substrate adhesion is unique. Before going into detailed description of experiments and analysis of results, it is important to have a quick look at the working principle of this method. Figure 4.10 presents a schematic flow chart of the process, for easy understanding of the principle.

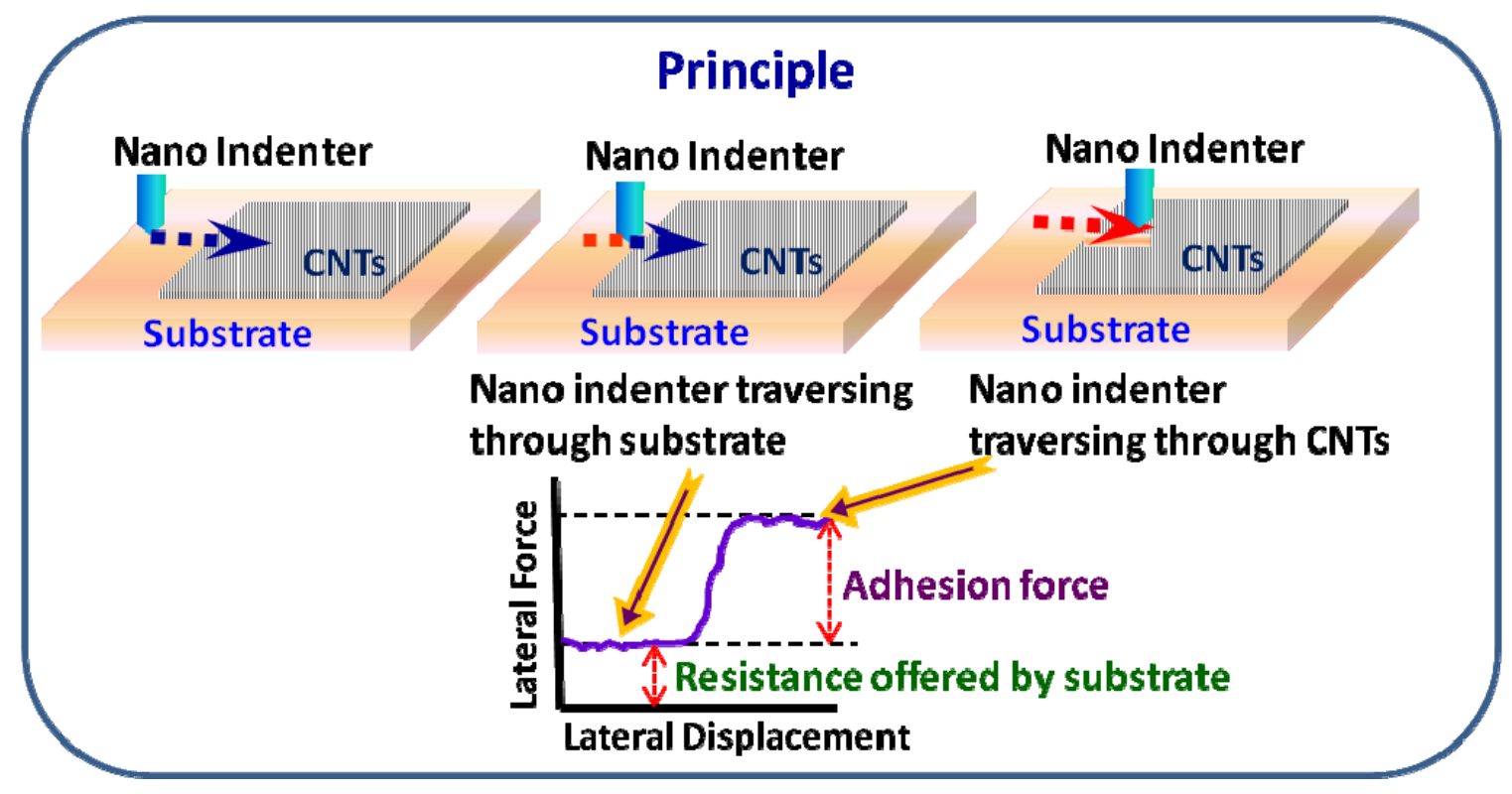

Figure 4.10: Schematic diagram of the working principle of nano-scratch technique.

During the process of nano-scratch, a Berkovich indenter was used (figure 4.11). The indenter first was moved to a point on the sample which was a bare substrate. Then it was programmed to move forward with a constant normal load of $150 \mu \mathrm{N}$, for a distance 
of $15 \mu \mathrm{m}$. During this travel, the indenter encountered CNTs and uprooted them from the substrate. A corresponding load increment could be observed in the lateral force vs lateral displacement plot, which was used further for quantifying the bonding energy of CNTs with the substrate. It is very much clear at this point that the nature of the later forcedisplacement plot will be dictated by the nature of the sample. Since, the present study focuses on dense, randomly oriented MWCNTs on different substrates, nano-scratch was primarily used for this kind of structures. However, to prove high resolution of the method, same technique was used for individual carbon nanocone (CNC) structure. Following sub-sections will provide detailed results and analysis of the nano-scratch study, performed on these two different types of structures.
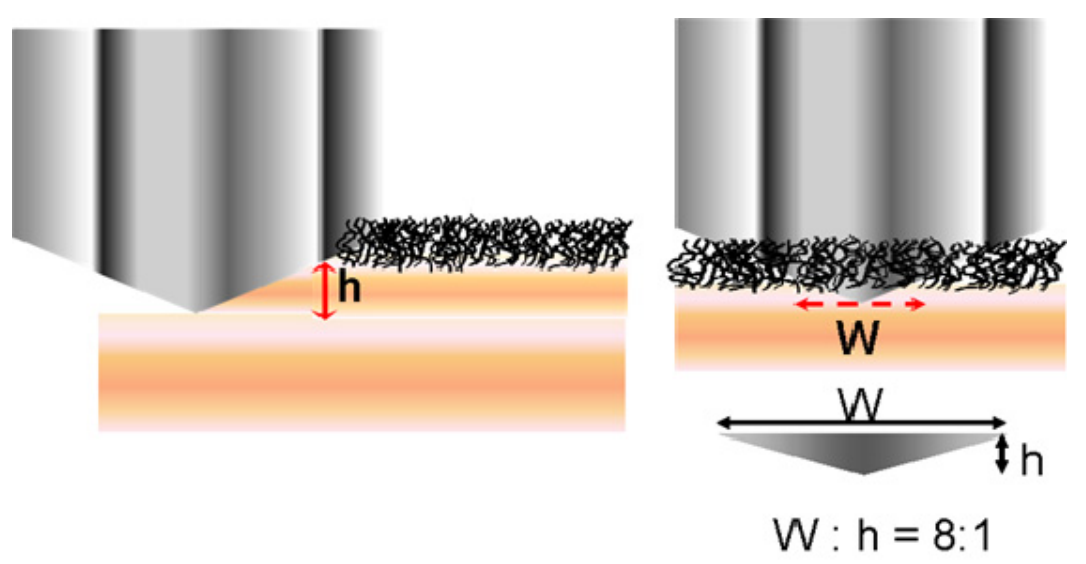

Figure 4.11: Schematic drawing and important geometric ratios of the Berkovich indenter used during nano-scratch study.

\subsubsection{Bonding Energy for MWCNT Structure}

As demonstrated in section 4.1, selection of substrate is one of the important issues to control field emission response of CNTs grown on them. To better understand 
this issue, nano-scratch tests were performed on two different MWCNT samples - using the combinations of $\mathrm{Cu}-\mathrm{Ti}-\mathrm{Ni}$ and $\mathrm{Si}-\mathrm{Ti}-\mathrm{Ni}$ (substrate-interlayer-catalyst). Both the samples had densely grown, randomly oriented MWCNT structure on them (figure 4.1 and 4.2). Detailed structural information could be found in section 4.1. For the nanoscratch study, three structural parameters were important - diameter, length and density of CNTs. Figure 4.12 presents diameter and density of CNTs, on $\mathrm{Cu}$ and Si substrates, as a function of CVD growth time.

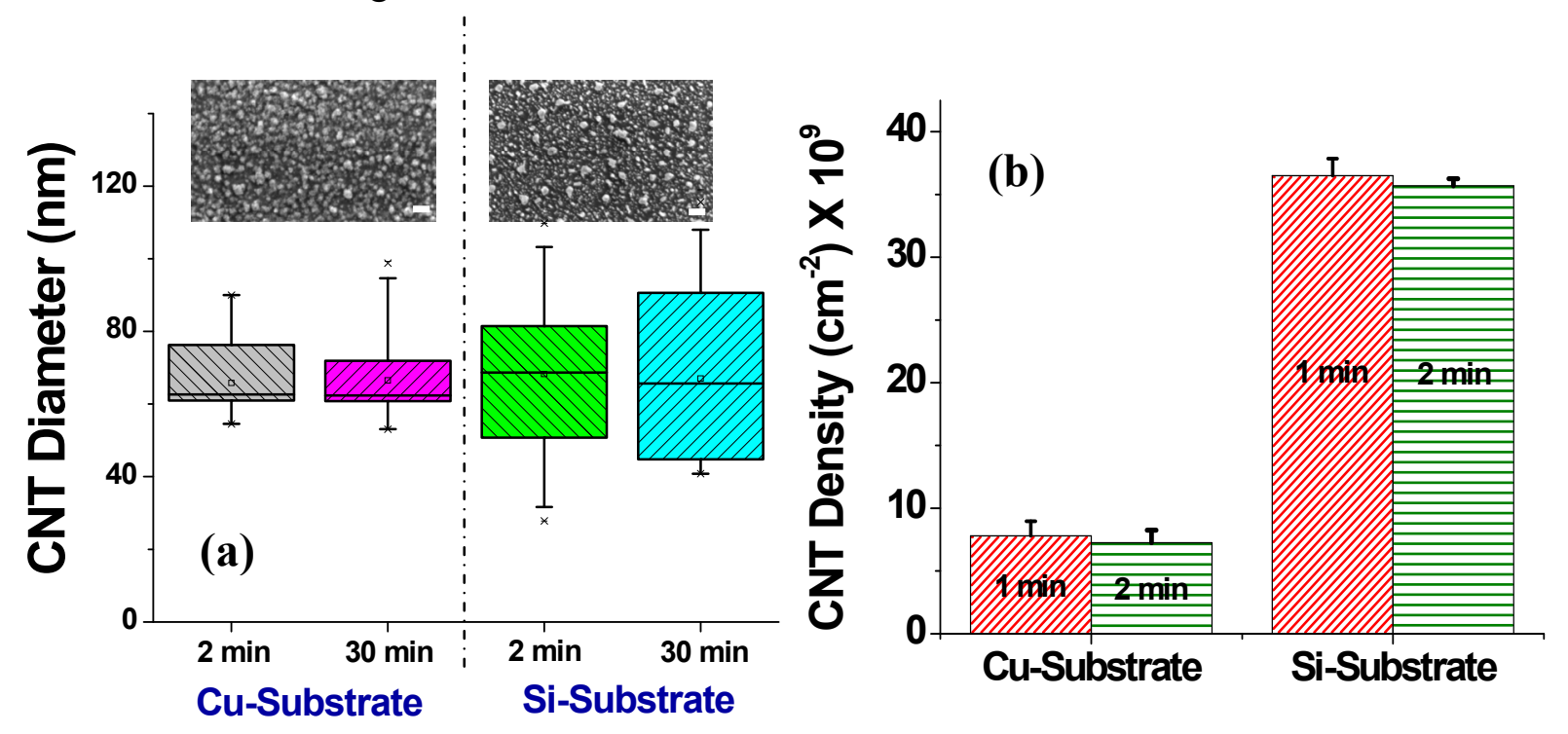

Figure 4.12: Structural features of CNTs on $\mathrm{Cu}$ and Si substrates. (a) Plot of diameter distribution of CNTs on both substrates, at two different growth time, 2 and 30 minutes. Insets show SEM images of catalytic islands on both samples (scale bar length is 200 nm). (b) Density of CNTs for both the samples, after 1 and 2 minutes of CVD growth.

Figure 4.12 (a) presents a quantitative estimation of the diameters of CNTs on both substrates as a function of growth time. It can be seen that the diameter of CNTs was almost independent of growth time, irrespective of substrates, although CNTs on $\mathrm{Si}$ 
substrate showed much wider diameter distribution. This fact is further supported by the observation reported by Bedewy et al. who have noted a maximum of $5-10 \%$ change in CNT diameter during growth period [98]. Moreover, in line with the in situ observation of CNT nucleation by Hofmann et al. [99], the present study also showed that CNT nucleation was almost instantaneous and only the growth in the longitudinal direction predominated with growth time. It should be mentioned here that due to random orientation of the MWCNTs, length of them could not be measured. However, keeping in mind about their possible effect on nano-scratch data (extra resistance provided by longer CNTs), nano-scratch tests were performed for samples with two different growth time -2 and 30 minutes.

Though during growth process, some CNTs may terminate or de-bond from the substrate abruptly $[98,100]$, CNT density was not found to change much with growth time. To gain further insight into this aspect, CNT densities were measured for both types of samples, after 1 and 2 minutes of growth time (Figure 4.12b). CNT density was found to remain nearly constant with growth time, although it was higher for the Si substrate, as compared to the $\mathrm{Cu}$ substrate. This difference in density of CNTs on $\mathrm{Cu}$ and Si substrates may be correlated with the distribution of catalytic nano-islands on these two surfaces (insets of Figure 4.12 a). It is well-known that the catalytic island formation initiates by dewetting from the substrate surface and final shape, size and crystallinity of these islands depend strongly on the minimization of surface and interface energy [36]. Initial thickness of the catalyst film also plays an important role [101]. Another important factor to explain variation in catalytic island number and size distribution may be differential 
solubility of catalytic layers in $\mathrm{Cu}$ and $\mathrm{Si}$ substrates. Higher solubility of $\mathrm{Ti}$ in $\mathrm{Cu}(1 \mathrm{wt} \%$, as compared to zero solubility of $\mathrm{Ti}$ in $\mathrm{Si}$ [33], at growth temperature) leads to onset of inter-diffusion and imposes additional constraints for breakage of the thin film, resulting in formation of less number of nano-islands. However, in absence of any such factors for $\mathrm{Si}$ (due to almost zero solubility of the catalyst in $\mathrm{Si}$ [33]), catalytic island formation is solely governed by wetting, presence of surface defects etc. and lead to a wider distribution. After clarifying all the structural issues, like diameter, length and density of CNTs on these two different substrates, nano-scratch tests were performed for quantifying the bonding between CNT-substrates.

Nano-scratches, using a standard Berkovich indenter (with $100 \mathrm{~nm}$ tip radius), were made in such a way that each scratch started from a bare surface and then traversed through the CNT forest, using a constant normal load (see section 3.7.3 and Figure 4.10). While moving through the CNTs, the indenter tip faced an extra opposing force, which was reflected through an increased lateral force on the tip. To calibrate the samples with respect to the contributions of substrates and the catalyst thin films on the lateral force behavior, comparative nano-scratch tests were conducted on bare substrates, substrates after thin catalyst film deposition and finally, after CNT growth. Figure 4.13 presents scanning probe microscopy (SPM) images of scratches on these three different types of Si-substrates. Responses from these three types of samples were found to be clearly different. 


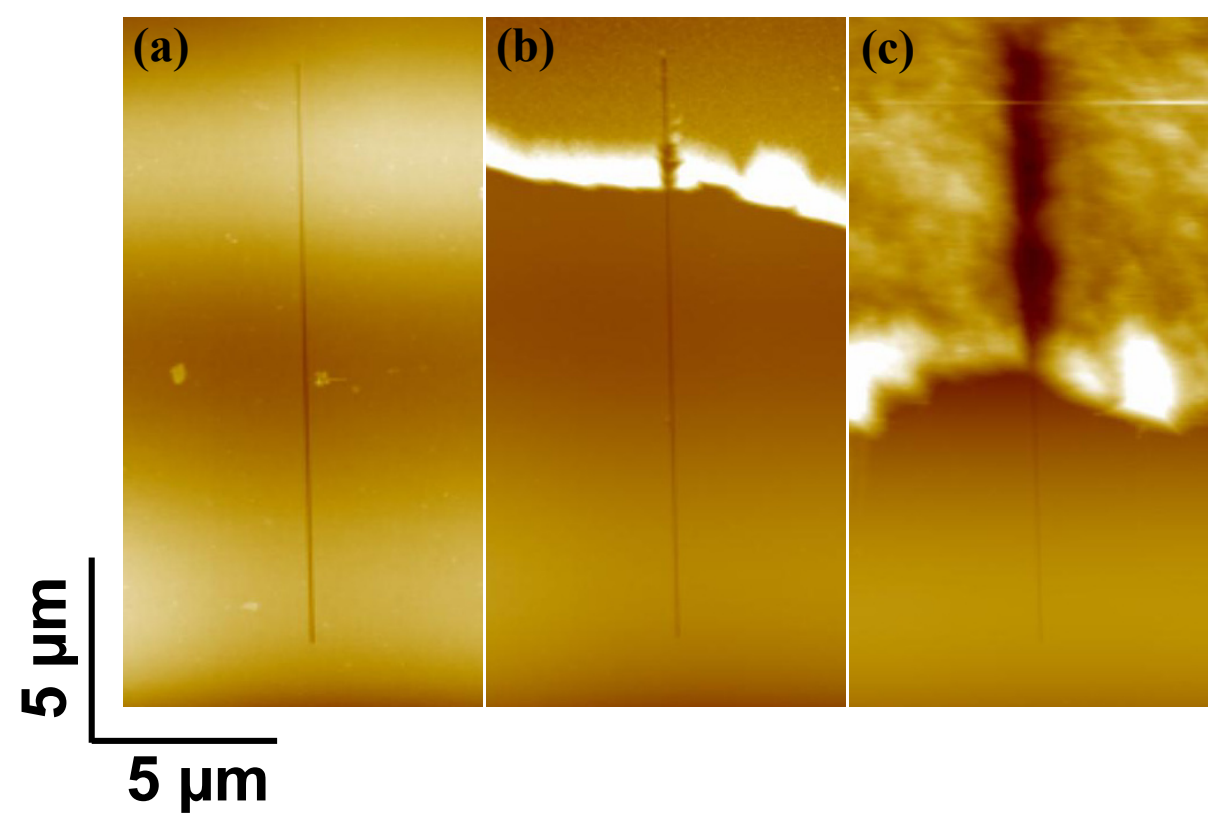

Figure 4.13: 2-dimensional scanning probe microscopy (SPM) images of the nanoscratches, made on samples with Si-substrate. From left to right, (a) scratches are visible on bare sample, (b) only catalyst deposited sample and (c) sample after CNT growth.

Nano-scratch plots, i.e., lateral force-displacement plots, are presented in figure 4.14 (a) and (b). These figures show that in presence of CNTs, the indenter tip experiences a much higher lateral force. Figures 4.14 (c) and 4.14 (d) (and their insets) show SEM images of the nano-scratch from $\mathrm{Cu}$ and $\mathrm{Si}$ samples, respectively (after CNT growth), indicating that CNTs were actually removed from the area of the scratches. Increased lateral forces for both samples were responsible to break the CNT-substrate bonds and to uproot the CNTs from the respective substrates. One important observation to be made from figure 4.14 (a) and (b) is the effect of Ti underlayer. Inability of Si and $\mathrm{Ti}$, to form a solid solution [33], led to retention of $\mathrm{Ti}$ as a separate layer (clearly visible as a separate layer in the SPM images presented in figure 4.15), which exerted an extra 
opposing force on the indenter. This force appeared as a peak in the lateral force curve for Si substrate (figure $4.14 \mathrm{~b}$ ). However, in the case of $\mathrm{Cu}$ substrate, good solid solubility of $\mathrm{Ti}$ in $\mathrm{Cu}$ leads to extensive inter-diffusion and thus, disappearance of any separate layer on $\mathrm{Cu}$ substrate.
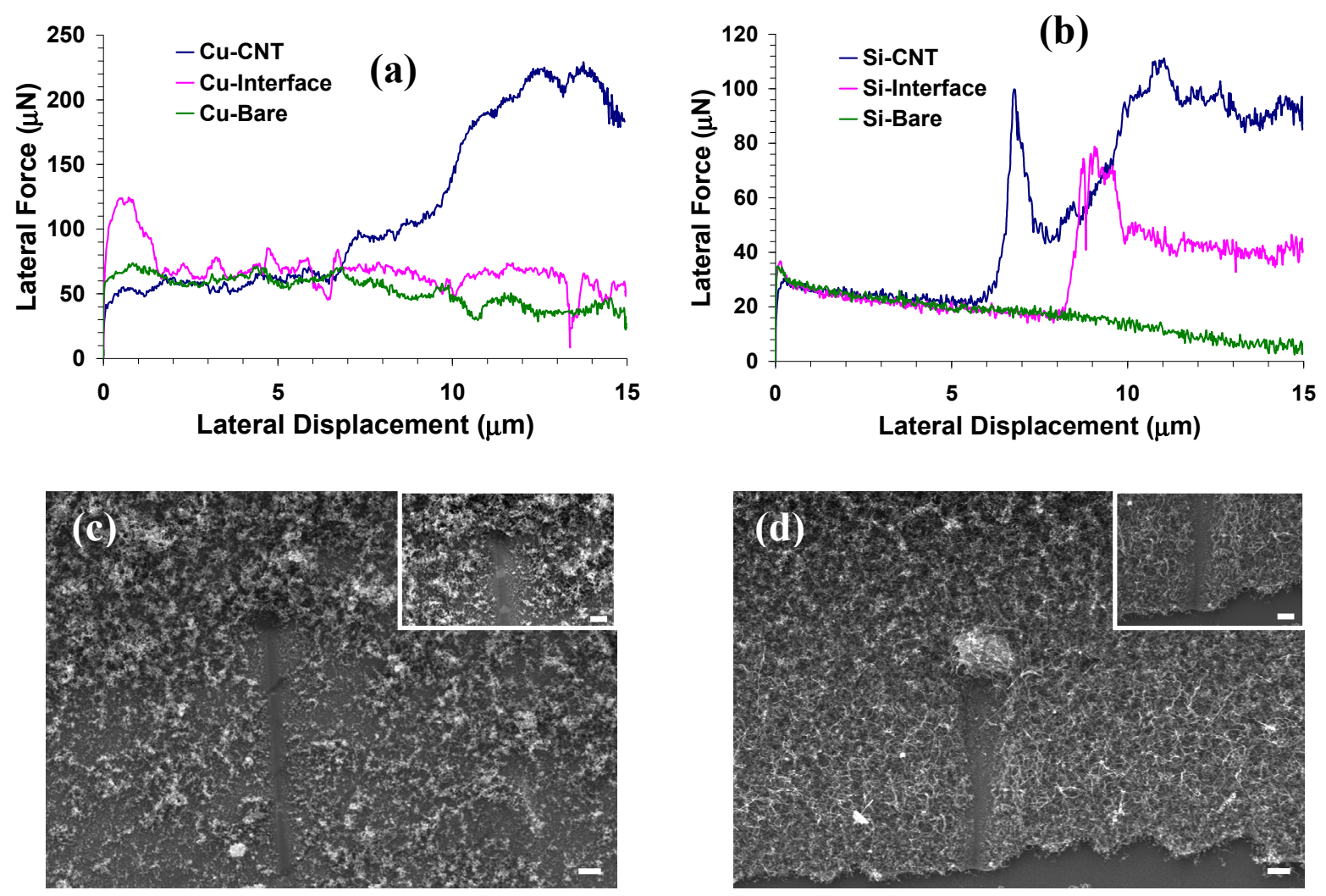

Figure 4.14: Nano-scratch through CNTs on different substrates. Lateral force response during nano-scratch tests on (a) Cu-CNT and (b) Si-CNT samples. Curves represent force required to scratch bare substrates, substrates with only catalysts and substrates after CNT growth. (c and d) SEM images of nano-scratches through the CNT structure on $\mathrm{Cu}$ and Si substrates, respectively. Insets show higher magnification SEM images of the scratches (scale bar length is $1 \mu \mathrm{m}$ ). 

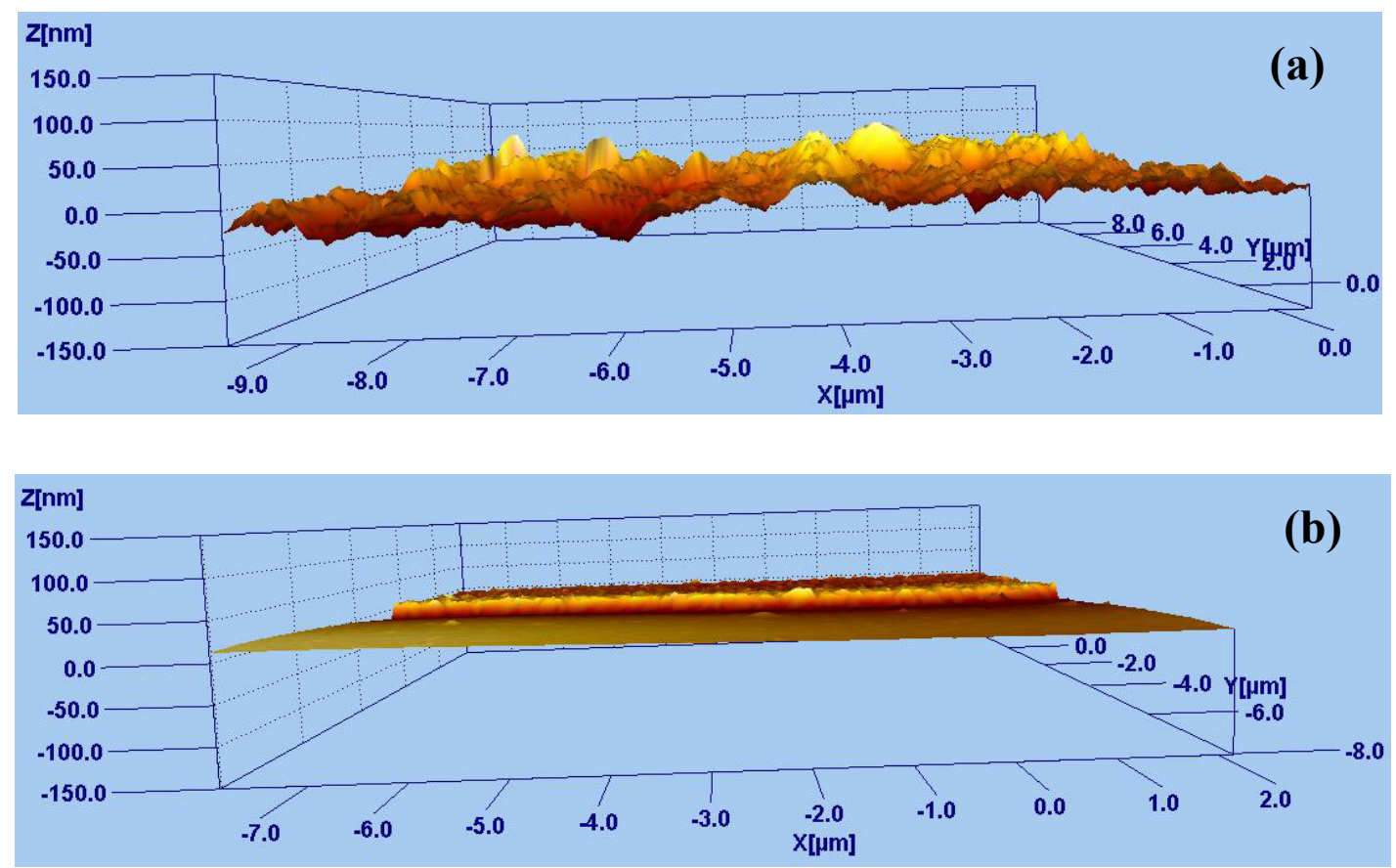

Figure 4.15: Scanning probe microscopy (SPM) images of the (a) $\mathrm{Cu}$ and (b) $\mathrm{Si}$ substrates after catalyst deposition.

Another important issue to be noticed is the effect of initial surface roughness of the substrates. $\mathrm{Cu}$-substrate had rougher surface as compared to the $\mathrm{Si}$ wafer (figure 4.15). This was reflected in the nano-scratch plots also (Figure $4.14 \mathrm{a}$ and $\mathrm{b}$ ) - scratching through the bare $\mathrm{Cu}$ substrate showed much more lateral force as compared to $\mathrm{Si}$ substrate. Thus, the nano-scratch technique was able to distinguish effects from substrate, catalyst and CNTs.

Increments in the lateral force, which can be interpreted as adhesion or bonding force of CNTs with their substrates, are plotted in figure 4.16, for five (5) scratches per sample and for two different growth times of 2 and 30 minutes (see appendix-1 for plots of all the nano-scratches). 


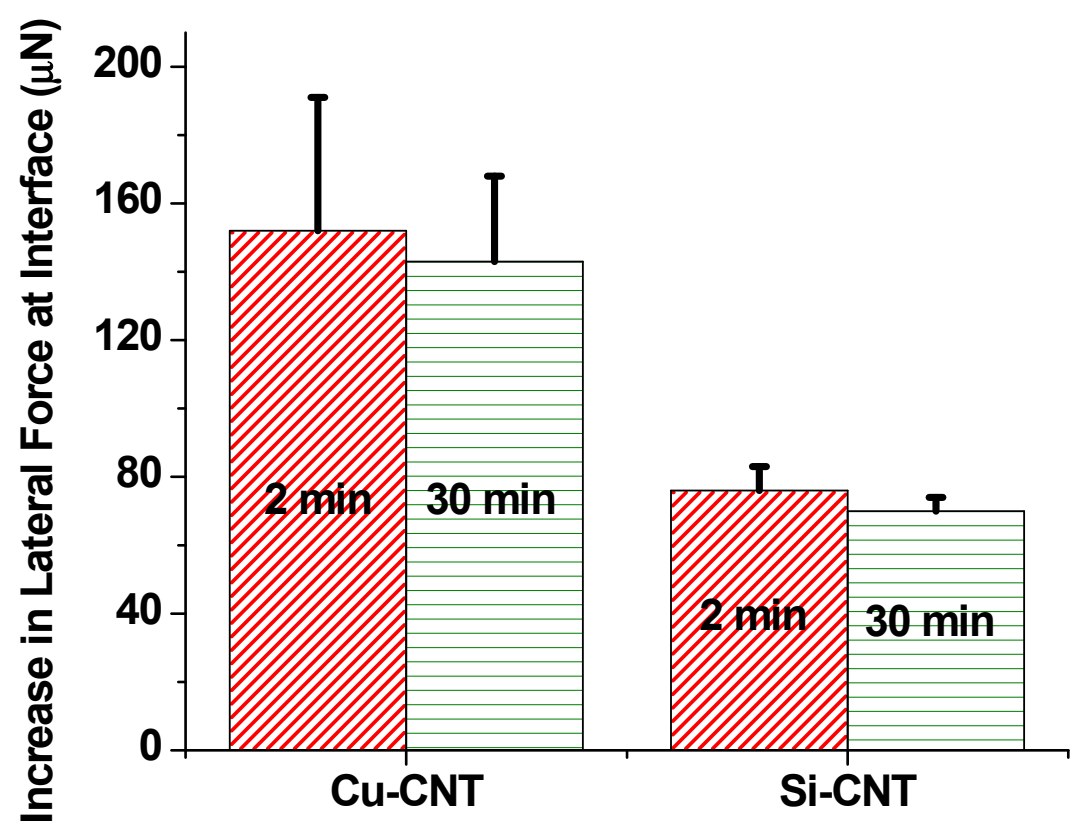

Figure 4.16: Plot of lateral force increment for both Cu-CNT and Si-CNT samples, after 2 minutes and 30 minutes of growth time.

It may be observed that growth time did not affect the lateral force in any significant way for both $\mathrm{Cu}$ and $\mathrm{Si}$ substrates. As higher growth time is related to longer CNTs, it seems that long CNTs, which formed a dense, tangled network, did not exert any extra load on the indenter. Hence, the increment in lateral force was mainly due to uprooting of CNTs from their substrates. Figure 4.16 also points out that the force required to de-bond $\mathrm{CNTs}$ from $\mathrm{Cu}$ substrate was much higher (almost 2 times) as compared to that of Si substrate. During CVD growth, Ti underlayer reacted with carbon precursor gas and formed $\mathrm{TiC}$, providing a strong bonding between the underlayer and the CNTs. However, to achieve better adhesion of CNTs to the substrate, it is necessary that the substrate and the underlayer form a strong bond. Comparison of $\mathrm{Cu}-\mathrm{Ti}$ and $\mathrm{Si}-\mathrm{Ti}$ phase diagrams indicates that solid solubility of $\mathrm{Ti}$ in $\mathrm{Cu}$ may promote formation of such 
bond between $\mathrm{Cu}$ and $\mathrm{Ti}$, while nil solubility of $\mathrm{Ti}$ in Si may prevent formation of any such bond [33]. However, interfaces need to be studied in detail for a better understanding of this issue.

After quantifying the forces required to debond a dense forest of CNTs from $\mathrm{Cu}$ and Si substrates, an effort was made to illustrate the bonding energy of individual CNT with the substrates. For this calculation, average densities of CNTs were taken for all samples. Due to extensive entanglement, CNTs could not be counted for samples having growth time more than 2 minutes during the present study. It is observed that till 2 minutes growth time CNT density remained almost constant (figure 4.12 b) [98]. Therefore, it was assumed that CNT density remained constant throughout the growth period. Though self-termination (sudden and sharp fall in growth rate) was reported to be observed by some researchers during CNT growth [98], which may decrease CNT density, it was found to occur at higher temperatures (> 1283 K) [100]. Moreover, time to initiate 'self-termination' was strongly dependent on growth temperature and found to increase sharply with lower growth temperature. During the present study, CNT growth was performed at much lower temperature of $973 \mathrm{~K}$. Following the trend reported in reference [100], it is expected that the effect of self-termination on CNT density would be minimum during the present study. However, nano-scratch tests were performed on 2minutes CNT grown samples also, for which CNT density was calculated (in addition to 30-minutes CNT grown samples) and results from both types of samples were compared. Figure 4.17 presents the results. 


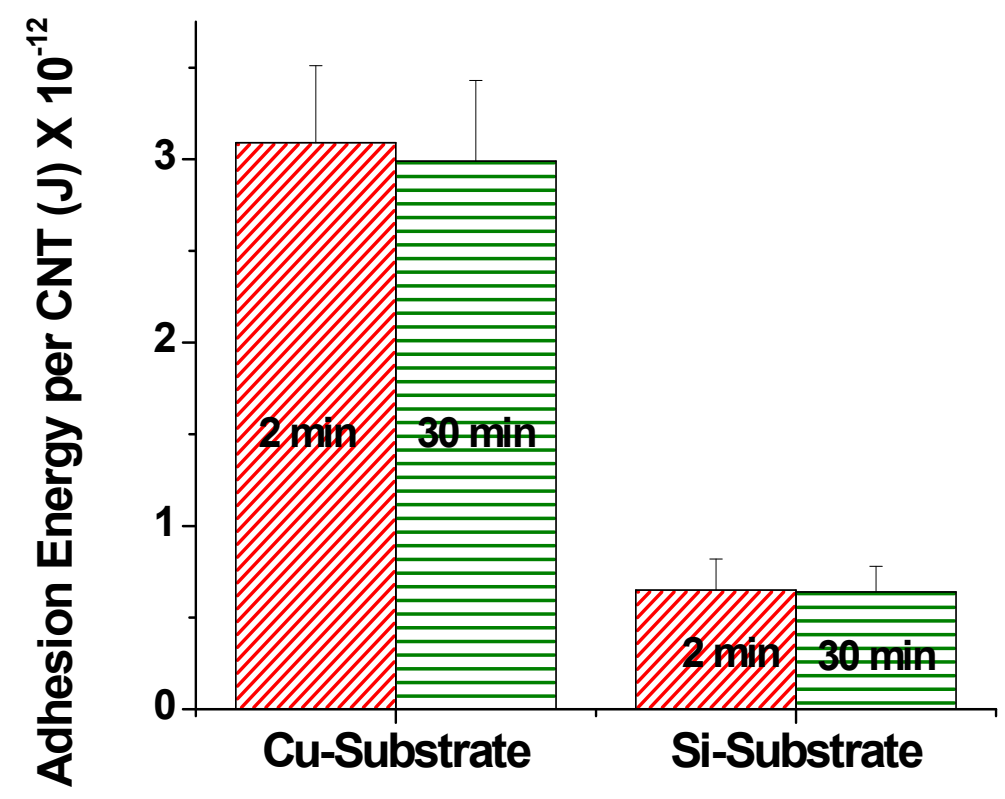

Figure 4.17: Comparison of adhesion energy of individual CNTs on both $\mathrm{Cu}$ and $\mathrm{Si}$ substrates.

The procedure to calculate adhesion energy of single CNTs is strongly dependent on the indenter geometry (figure 4.11) and is given in detail in appendix-2. Figure 4.17 is generated from this calculation procedure. As the diameter of CNTs remains almost constant but length increases with growth time, the difference in bonding energy of 2 vs 30 minutes grown samples can be related to the effect of CNT length. This difference was found to show a variation of $1-2 \%$ only for both $\mathrm{Cu}$ and $\mathrm{Si}$ substrates, while the actual calculated debonding energy values range over $\pm 15 \%$ of the mean. Hence, the CNT length effect on the debonding energy is considered to be negligible. The energy calculated thus represents the energy required to de-bond a single CNT. It may be observed that $\sim 3 \mathrm{pJ}$ energy is required to debond one single $\mathrm{CNT}$ from the $\mathrm{Cu}$-substrate, which is equivalent to breaking 5 million $\mathrm{C}-\mathrm{C}$ bonds [102]. It may be noted here that the adhesion energy for $\mathrm{CNTs}$ on $\mathrm{Cu}$-substrate is $\sim 4.7$ times higher than that of Si-substrate, 
while the debonding force was nearly 2 times higher for CNTs on $\mathrm{Cu}$-substrate than that on Si-substrate. This difference in the factor is due to CNT density difference among both the samples. It will be appropriate to mention here that the bonding energy of single CNTs, as calculated above, is true only for the materials and methods used in the present study and is expected to vary depending up on the experimental conditions followed.

Nano-scratch tests performed on both $\mathrm{Cu}$-Ti-Ni-CNT and Si-Ti-Ni-CNT samples have shown marked differences in CNT-substrate bonding energy, which can be logically related to the structure beneath CNTs. In line with the observations made in section 4.1, it is evident that interface engineering plays extremely important role and $\mathrm{Cu}-\mathrm{Ti}-\mathrm{Ni}$ combination presents itself as a good combination of substrate-interlayer-catalyst for growth of strongly bonded CNTs, which can be used successfully for devices such as field emitters.

After successful demonstration of bonding energy of single CNTs in a mat of randomly oriented CNTs, further effort was made to prove high resolution of this technique by using $\mathrm{CNC}$ structure, as described in the following sub-section.

\subsubsection{Bonding Energy for Carbon Nanocone Structure}

In order to show the ability of nano-scratch technique to directly quantify adhesion energy down to single nanostructure level, an explicitly different 1-D nanostructure - a periodically spaced and vertically aligned carbon nanocone (CNC) structure grown on a lithographically pre-patterned Si substrate, was further considered [103]. Figure 4.18 presents SEM images of this structure. 

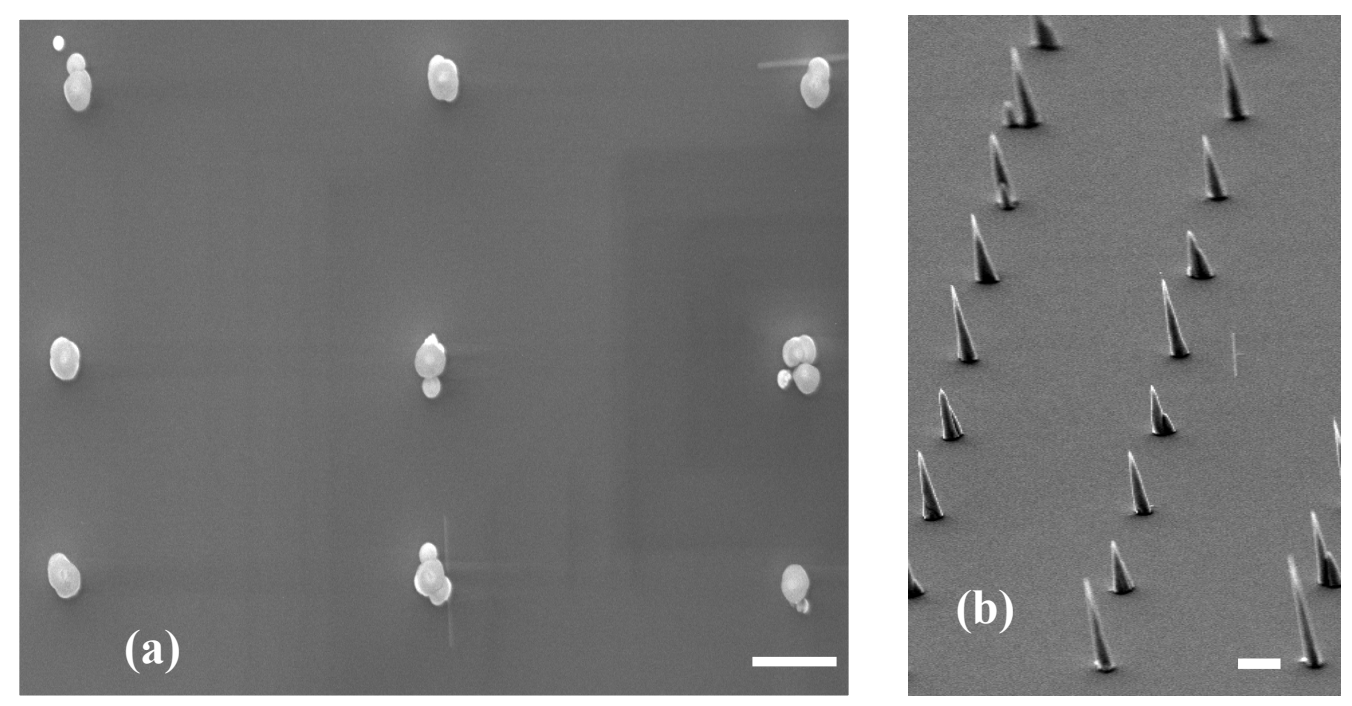

Figure 4.18: SEM images of nano-patterned carbon nanocone structure - (a) before nano-scratch, imaged from top, showing presence of multiple CNCs in each catalytic islands and (b) before nano-scratch, imaged at a tilt angle of $\sim 40^{\circ}$. All scale bars in this figure are $1 \mu \mathrm{m}$.

The figure shows typical SEM images of the structure, where each catalyst island is observed to accommodate 1-3 CNCs. Figure 4.18 (b) shows some of the islands having single carbon nanocone only. Each island is $\sim 300 \mathrm{~nm}$ in diameter and the CNCs formed on these islands are $\sim 2 \mu \mathrm{m}$ in length. Nano-scratches were performed on this sample in such a way that these scratches traverse through the nanocone structures. During the scratching, CNCs were uprooted by the scratching force and gave us a directly measured value of bonding energy. The result of the test is given in figure 4.19, clearly showing uprooting of CNCs (in SEM image) and corresponding lateral force increment in the lateral force vs lateral displacement plot. 
(a)
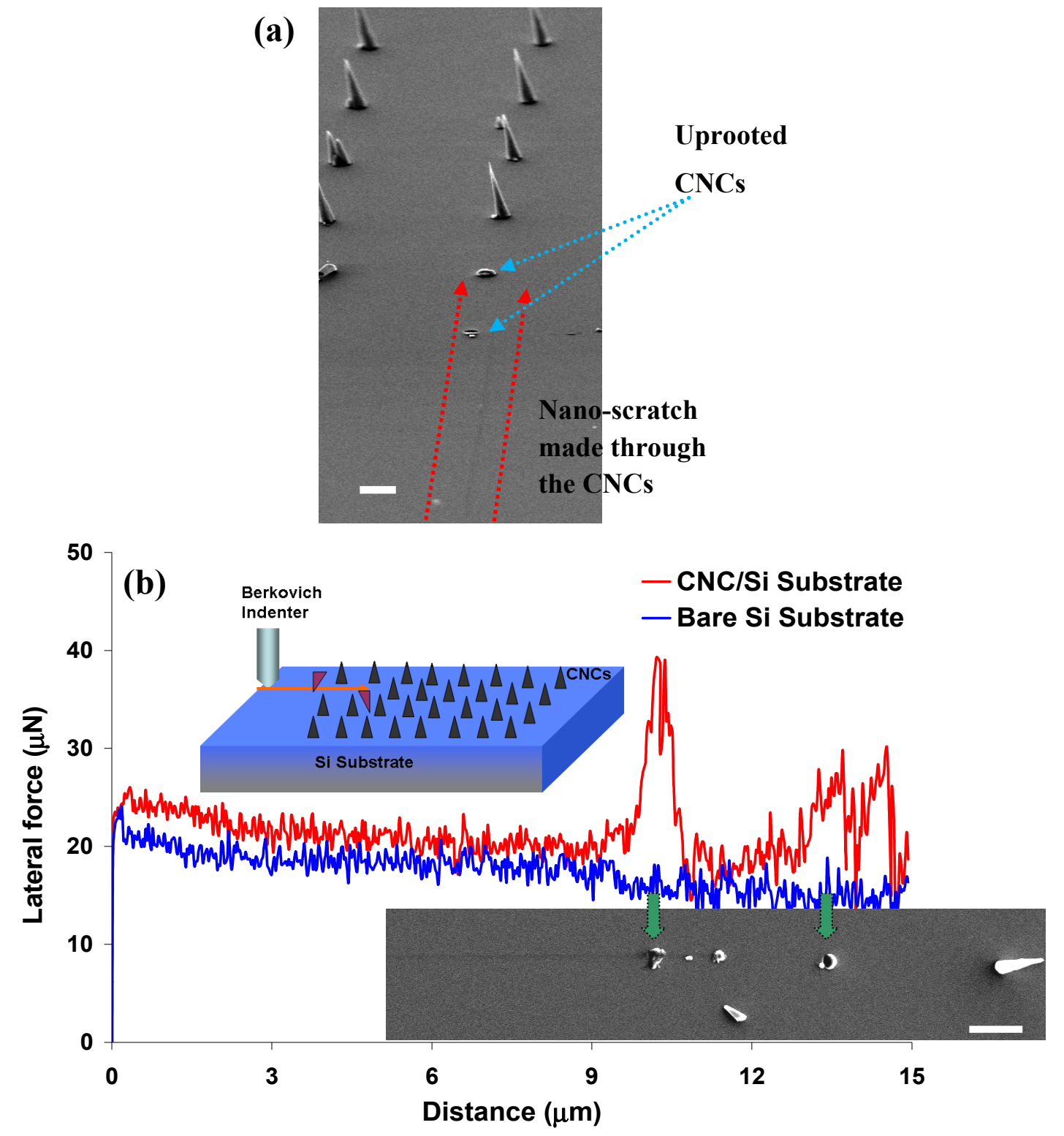

Figure 4.19: Nano-scratch on nano-patterned carbon nanocone structure. (a) SEM image of the CNC structure after nano-scratch, showing removal of CNCs, imaged at a tilt angle of $\sim 40^{\circ}$. (b) Lateral force response during nano-scratch test on Si-CNC sample, comparing effects on bare substrate and through CNCs. The upper inset shows a schematic of the process, uprooted CNCs being shown in a different color. The lower inset shows corresponding SEM image. All scale bars in this figure are $1 \mu \mathrm{m}$. 
Nano-scratches made on this sample clearly showed uprooting of individual CNCs, both in SEM images and in corresponding quantitative data. Appearance of peaks in nano-scratch plot (Figure 4.19 b) could easily be correlated with the distance between consecutive nanocone islands. Separate peaks for each island of nanocones showed high resolution of the nano-scratch technique to quantify de-bonding force for single nanocone structure. Force required to debond CNCs in each island was found to vary between 10$25 \mu \mathrm{N}$, depending on the number of CNCs present in each island. This corresponds to a debonding energy of 8-10 $\mathrm{pJ}$ per $\mathrm{CNC}$. However, it may be noted here that debonding energy for CNCs includes the effect of catalysts. Synthesis techniques and parameters used for CNCs and CNTs (as mentioned in previous sub-section) were totally different. Furthermore, CNCs had a much higher contact area (having a solid base diameter of $\sim$ $300 \mathrm{~nm}$ ) than the CNTs (ring-type base, with a diameter in the range of $60-80 \mathrm{~nm}$ ). As mentioned before, CNT-substrate bond is a strong function of the properties of materials, synthesis conditions and their exact contact area. Thus, debonding energy values, as measured for the CNCs and CNTs, are not directly comparable. However, nano-scratch test on CNC sample shows its direct application in single nanotube/nanowire samples.

Interface engineering seems to play a very crucial role in determining performance of CNT based devices. Nano-scratch technique now can act as a quantitative tool to describe CNT-substrate bonding energy and hence, can be related to device performance and reliability. In the following section, a completely different geometry (3dimensional structure) of CNT based field emitter will be presented, which also incorporates the lessons learnt from sections 4.1 and 4.2. 


\subsection{Structure and Field Emission Performance of 3-Dimensional Field Emitter}

Though CNTs have become widely popular as a potential material for field emitters, future devices require further enhancement in emission current density and emission stability and a simpler processing technique for efficient fabrication of CNTbased field emission cathodes [8]. Current research activities on CNT-based field emitters were focused to address these issues. The simplest way to enhance emission current from CNT cold cathodes could be increasing the density of active CNT emitters present within a specific area. However, screening effect from closely spaced neighboring CNTs is known to adversely affect the field emission properties, which limits the maximum allowable active CNT emitters without deteriorating the emitter performance [44]. Another important issue for electron sources used in high power microwave (HPM) devices and other high current applications is structural stability at high operating electric fields, to obtain higher emission current. Operating the field emitter at higher electric field may cause ion bombardment and arcing, especially from highest localized electric field areas of protrusions, loosely bonded absorbents/contaminants. This may cause significant damage to the CNT structure and could lead to permanent impairment of the emitters. These issues can be addressed well in a three-dimensional (3-D) design of the field emitter structure, in which CNT emitters are embedded within microchannels.

As schematically shown and described in section 3.2.2, the 3-D design incorporates specific number of micro-channels within a particular surface area of a twodimensional (2-D) substrate. Creation of the micro-channels offers increment in total surface area (owing to extra side walls of the channels) available for CNT growth, without consuming more 2-D space (foot-print area). Increased surface area depends on 
the size and number of the channels and the thickness of the substrate being considered. Figure 4.20 shows a graphical representation of this fact, for different channel diameters and substrate thickness, assuming a fixed foot-print area of $1 \mathrm{~mm}^{2}$ and incorporating maximum number of channels within this area. The scheme of calculation, used in creating this plot, is based on geometry of the system and is explained in detail in appendices (appendix-3).

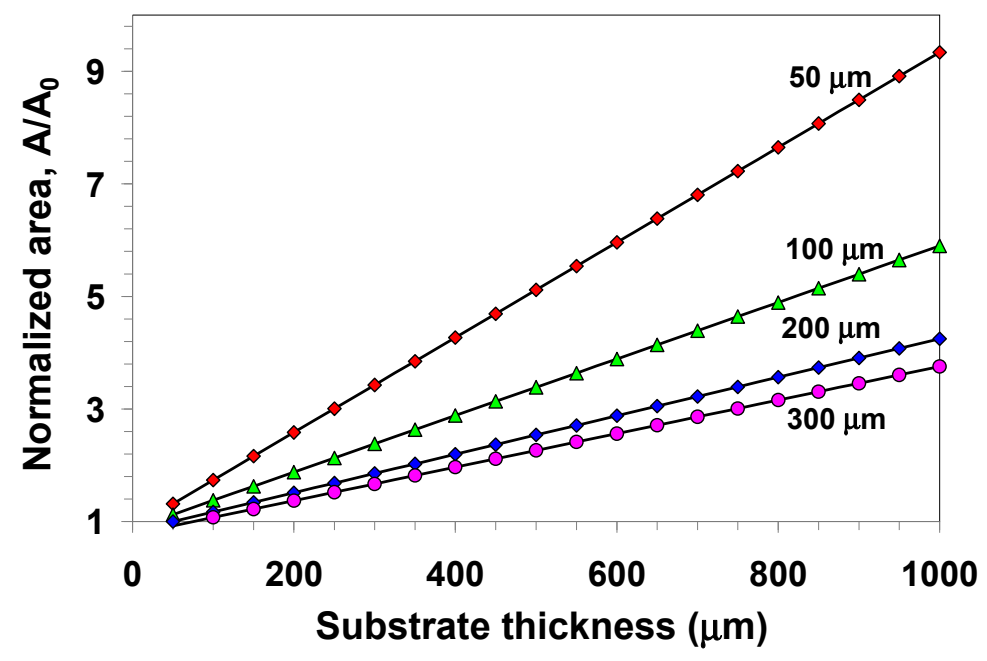

Figure 4.20: Representation of the effect of substrate thickness and channel diameter on total active surface area available for CNT growth (values mentioned against each line in the plot is the channel diameter).

It is evident from this plot that higher surface area enhancement could be achieved by creating finer channels in a thicker substrate. Due to enhanced surface area and growth of CNTs within channels, these 3-D cathodes offer higher emitter density in a specific area and hence, are expected to enhance field emission response, as compared to conventional 2-D cathodes. In a previous study by Seelaboyina et al. [8], it was shown 
that such a 3-D field emitter, created on alumina substrate, offers better emission response compared to its 2-D counterpart. However, low thermal and electrical conductivity of alumina $\left(29-33 \mathrm{~W} / \mathrm{m} . \mathrm{K}[104,105]\right.$ and $1 \times 10^{-15} \mathrm{~S} / \mathrm{cm}[106]$, respectively) may affect heat transfer and electron movement during field emission and finally, on the structural stability and emission current, respectively. In the previous sections, it was shown that an interface-controlled growth of MWCNTs on $\mathrm{Cu}$ substrate offers best field emission response and emission stability, when compared to other samples. Using the lessons learnt from those studies, hence, a synergistic effect of the advantages offered by 3-D emitter structure and interface-controlled MWCNT emitter on $\mathrm{Cu}$ substrate was proposed, which could lead to much better field emission response. Thus, MWCNTbased 3-D microchannel cathode structure on $\mathrm{Cu}$ substrate was fabricated. Field emission response from this structure will be presented in the following sub-sections.

\subsubsection{Effect of Number of Micro-Channels on Field Emission Performance}

To study the effect of number of micro-channels on field emission behavior, three different types of samples were prepared with 4, 9 and 16 micro-channels, respectively. Structure of the 3-D field emission device is presented in figure 4.21. Micro-channels, before CNT growth, are observed in figure 4.21 (a) As can be seen from figure 4.21 (b) and (c), dense CNT forest type structure was grown on the substrate, surrounding the micro-channels, including the walls of the channels. CNTs have length of approximately $20 \mu \mathrm{m}$ and diameter in the range of 80-120 nm. Raman spectra, taken from the samples

(figure $4.21 \mathrm{~d}$ ), showed high $\mathrm{D} / \mathrm{G}$ peak intensity $\left(\mathrm{I}_{\mathrm{D}} / \mathrm{I}_{\mathrm{G}}\right)$ ratio, indicating presence of defects in the CNT structures. The structure was found to be quite similar to those found 
in 2-dimensional field emitters and a detailed description about interpretation of Raman spectra is given in section 4.1.1.

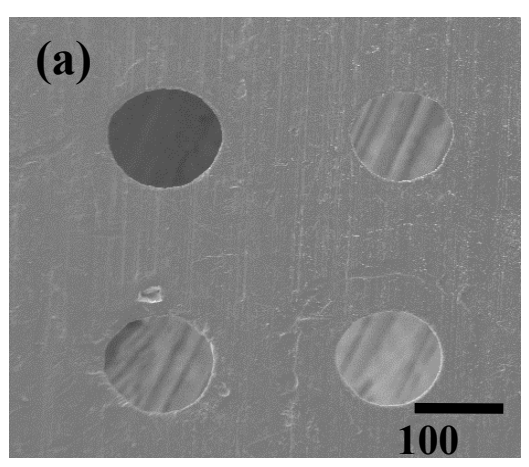

$\mu \mathrm{m}$

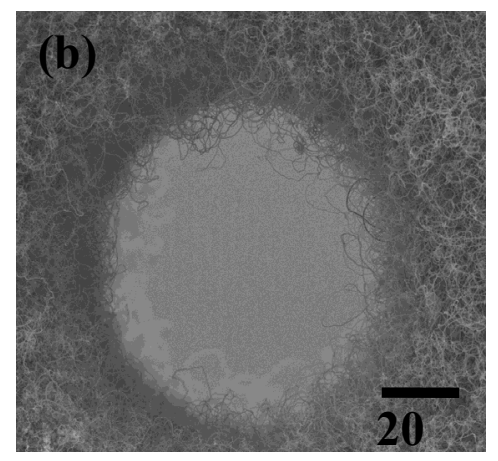

$\mu \mathrm{m}$

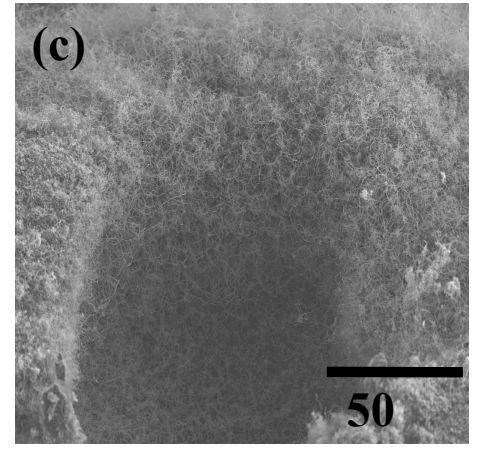

$\mu \mathbf{m}$

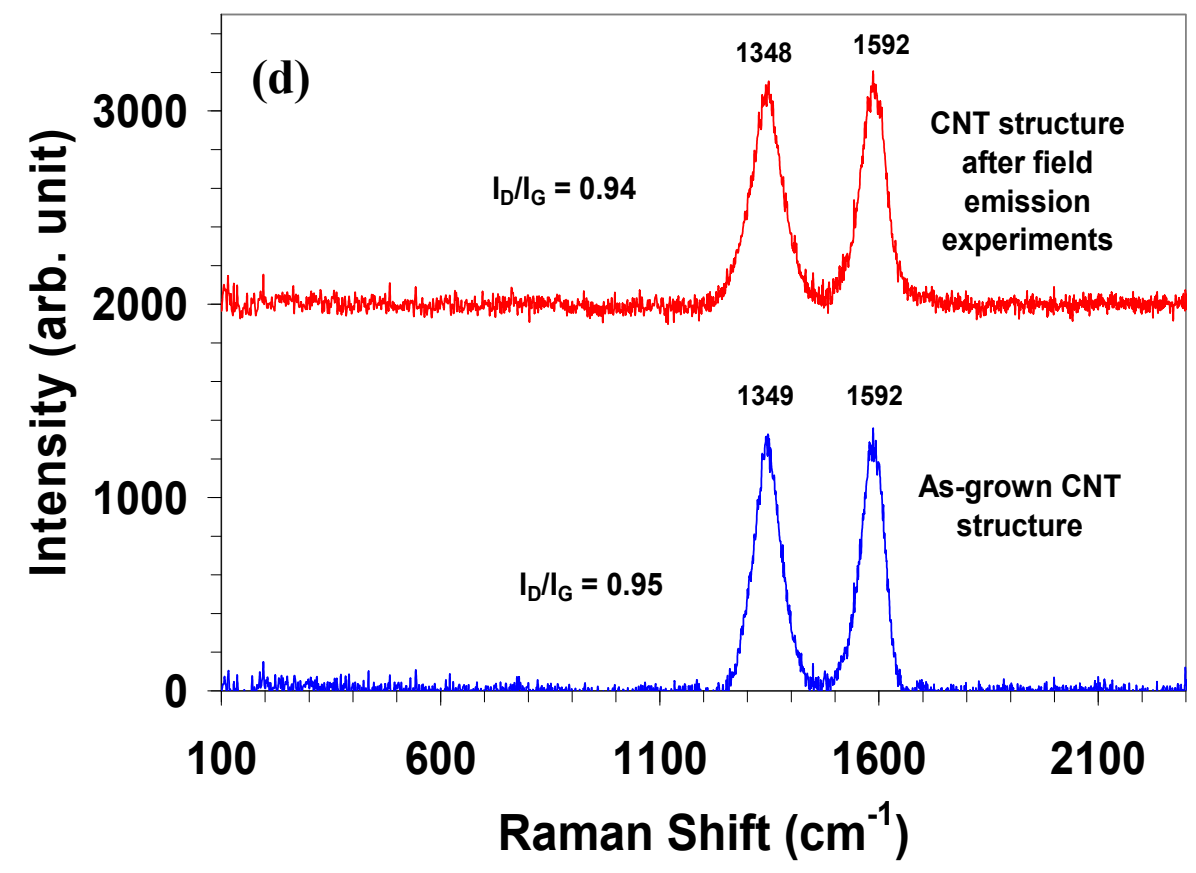

Figure 4.21: Structure of the 3-D field emission device. (a) Micro-channels created on the Cu substrate. (b) MWCNTs synthesized around and (c) within the micro-channels. (d) Comparison of Raman spectra, before and after field emission tests, showing $D$ and $G$ peaks - signature peaks for CNTs.

Field emission responses of the CNT based 3D emitters, with varying number of micro-channels, are presented in figure 4.22 (next page). 

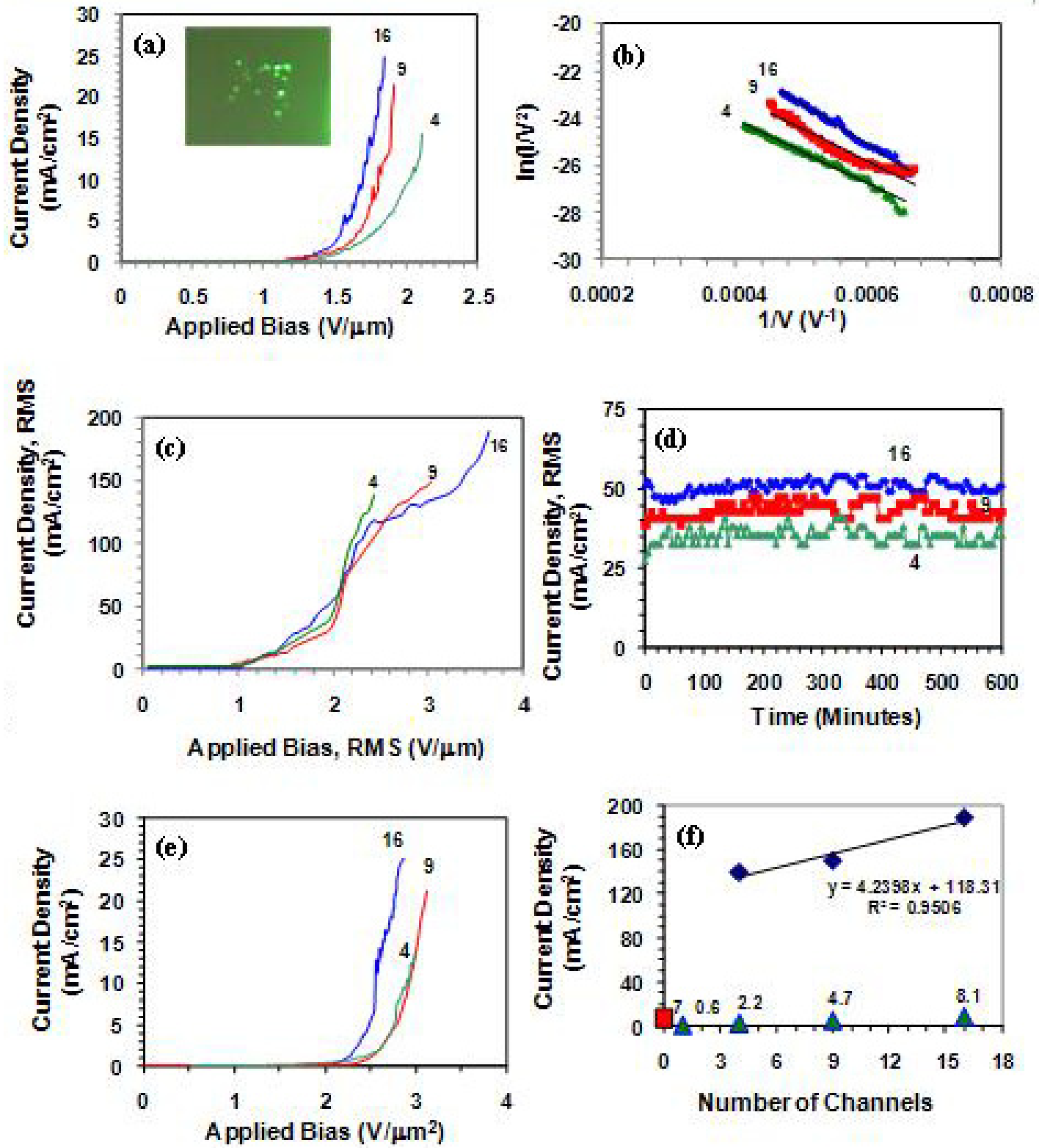

Figure 4.22: Field emission response of the 3-dimensional cathode structure (numbers mentioned within the plots are the number of micro-channels in corresponding samples). (a) Field emission current density from the emitters having different number of channels, under DC bias. Inset shows field emission image, captured using green phosphor coated ITO glass as anode. (b) Fowler-Nordhiem (F-N) plot of the emitters showing straight line behavior. (c) Field emission current density from the emitters having different number of 
channels, under AC bias. (d) Stability of emission during continuous, long-time operation, under AC bias. (e) Field emission response of emitters under DC bias, after 10 hours of exposure during stability test, showing emitter structure stability. (f) Comparison of current density from 2-D and 3-D CNT based emitters ['red square': data for 2-D CNT based emitter on Cu substrate (taken from section 4.1); 'green triangles': data from 3-D CNT based emitter on alumina substrate (taken from Seelaboyina et al, ref. 8)].

While figure 4.22 (a) shows the current density vs. applied bias (J-E) curves for the emitters, figure 4.22 (b) presents the conventional Fowler-Nordheim (F-N) plots both under DC bias. It could be pointed out from the J-E curves that emission current density increases with increasing number of micro-channels. The turn-on field ( $\mathrm{E}_{\mathrm{TO}}$, defined as the field required to produce $10 \mu \mathrm{A} / \mathrm{cm}^{2}$ emission current) was found to be quite low for all the emitters (irrespective of number of channels) - 1.1-1.2 V/ $\mu \mathrm{m}$. Though these values were higher as compared to previously reported $\mathrm{E}_{\mathrm{TO}}$ values for 2-D emitter structure (section 4.1), this behavior could be related to uncertainty in identifying exact inter-electrode distance, caused by the rare situation of 3-D structure (due to presence of CNT emitters within the channel wall). While all calculations were based on minimum inter-electrode distance (i.e. the distance between top surfaces of both the electrodes), actual scenario might be quite different, as CNTs were present over a range of inter-electrode distance (within the channels). In order to better understand this complex situation, simulation studies need to be performed in future, to predict electric field distribution along the channels. On the other hand, threshold field ( $\mathrm{E}_{\mathrm{th}}$, defined as 
the field required to achieve $10 \mathrm{~mA} / \mathrm{cm}^{2}$ current density) of these emitters were observed to vary in wider range -1.69 to $2.04 \mathrm{~V} / \mu \mathrm{m}$, depending upon number of channels. This can easily be correlated to the total number CNT emitters present in structure - samples with more channels have more emitters and hence, higher emission current density at lower excitation field. Further, straight line nature of all the F-N plots ensures that the emission, observed in this study, is a result of electron tunneling through MWCNTs.

Since continuous operation in DC bias can damage structure of the MWCNT emitters, AC bias (often with very low duty-cycle) is preferred for practical purposes. In the present study, a sinusoidal AC bias (50\% duty cycle) was used. Figure 4.22 (c) shows field emission response of the emitters under AC bias, as a function of number of microchannels present in the structure. Again, the field emission current was found to depend on the number of channels and the highest emission current density $\left(189 \mathrm{~mA} / \mathrm{cm}^{2}\right.$, RMS) was obtained from the sample having 16 channels. Current density obtained from this sample was very high and is considered as one of the best emission current densities, reported in open literature. Excellent emission behavior of these 3-D emitters can be related to the novel geometry of the device and to the low interface resistance. MWCNTs, predominantly being metallic in nature, created a metallic contact with $\mathrm{Cu}$-substrate and offered low contact resistance, facilitating easy electron transport and good emission properties.

Stability of the emitters is another extremely important issue for any practical application. It was found (figure $4.22 \mathrm{~d}$ ) that all the emitters showed very good stability over a continuous operational period of 10 hours, though at different emission current 
density levels. During the stability test also, the emitter with 16 channels showed much better performance as compared to other emitters, in terms of operating at a higher current level. Apart from the emission stability, structural stability of the emitters is another important factor for predicting life-time of field emission devices. Structural stability of the emitters was tested after the 10-hour long, continuous exposure of stability tests, in two different ways - field emission under DC bias and Raman spectroscopy. Figure 4.22 (e) shows the result of the field emission test. A quick comparison between figure 4.22 (a) and (e) shows that the emission current level was similar in both the cases, though the turn-on field was little higher for the latter case. This result indicates that damage to the emitter was not significant. This conclusion can further be supported from the Raman spectra, obtained from the samples, after stability test (figure $4.21 \mathrm{~d}$ ). Raman spectra obtained from the MWCNT structures, tested in as-grown condition and after finishing field emission experiments did not show any appreciable difference. As indicated in the figure, positions of $\mathrm{D}$ and $\mathrm{G}$ peaks and their relative intensity ratios $\left(\mathrm{I}_{\mathrm{D}} / \mathrm{I}_{\mathrm{G}}\right)$, for both the samples, remained almost same. This observation indicates that the rigorous field emission experiments could not induce much defect to CNT structures. In contrast to the observation made by Chen et al. [44], this 3-D MWCNT-based field emitter structures on $\mathrm{Cu}$ substrate, did not show any visible sign (e.g., partial uprooting) of structural damage. This fact may be related to good bonding between $\mathrm{Cu}$ and CNTs, as already pointed out in section 4.1 and 4.2. Moreover, an all-metallic structure from MWCNT to $\mathrm{Cu}$ substrate, through a metallic interface (Ti and Ni), aided in faster heat dissipation during field emission experiments and thus minimizing the chances of any structural damage. 


\subsubsection{Predicted Current Density}

The best advantage of the 3-D MWCNT-based emitters on $\mathrm{Cu}$ substrate is the high current density. Figure 4.22 (f) presents a comparison of emission current density among different varieties of 3D emitters (with 4, 9 and 16 number of micro-channels, within $1 \mathrm{~mm}^{2}$ foot-print area) and with those reported for 2-D emitter structure (MWCNTs-on-Cu, section 4.1) and 3-D emitter structure (CNTs on alumina microchannel plates [8]). The highest emission current density $\left(\sim 190 \mathrm{~mA} / \mathrm{cm}^{2}\right)$ observed for the sample having 16 micro-channels (in the present study), is actually more than an order of magnitude higher than all current density values reported in section 4.1 and reference 8 . This observation clearly points out to the benefits of the 3 -D emitter structure on $\mathrm{Cu}$ substrate. It may further be noted that variation in current density, as a function of number of channels, is almost linear (with more than $95 \%$ degree of fit). Following this linear trend, current densities of 3-D emitters of $\mathrm{Cu}$ substrate having higher number of channels, could be predicted (table 4.4). Current density, predicted for 1400 channels, $\left(6.054 \mathrm{~A} / \mathrm{cm}^{2}\right)$ is found to be well above the predicted current density values for 3-D alumina based CNT emitter $\left(708 \mathrm{~mA} / \mathrm{cm}^{2}\right)[8]$ and other literature reported values $(3.55$ $\mathrm{A} / \mathrm{cm}^{2}[44]$ and $4 \mathrm{~A} / \mathrm{cm}^{2}$ [107]). However, it may be noted here that the actual current density from such a sample might be limited by screening effect from neighboring CNTs. Limitation of our instruments, to measure such a high current, restricts us in providing experimental support to this issue. However, it is proposed at this point that detailed experimental and simulation is undertaken to clarify this issue. 
Table 4.4: Estimated current density from different number of channels (from $1 \mathrm{~cm}^{2}$ footprint area)

\begin{tabular}{|c|c|}
\hline $\begin{array}{l}\text { Number of } \\
\text { Channels }\end{array}$ & $\begin{array}{l}\text { Estimated Current Density } \\
\left(\mathbf{A} / \mathbf{c m}^{2}\right)\end{array}$ \\
\hline 100 & 0.542 \\
\hline 400 & 1.814 \\
\hline 625 & 2.768 \\
\hline 900 & 3.934 \\
\hline 1200 & 5.206 \\
\hline $1400 *$ & 6.054 \\
\hline
\end{tabular}

* Maximum number of channels that could be created within $1 \mathrm{~cm}^{2}$ area is 1412 , which is approximated as 1400 , here.

The results indicate that a proper 3-D design, on a suitable substrate, can lead to a big increment in current density, as compared to the 2-D emitter structures. In the present case, the 3-D emitter structure on $\mathrm{Cu}$ substrate with 16 channels showed a gigantic 27 times enhancement in current density, as compared to its 2-D counterpart. Further, selection of $\mathrm{Cu}$ over alumina and realization of interface-control (through $\mathrm{Ti}$ and $\mathrm{Ni}$ ) ensured a strong and low-resistance interface and contributed as another favorable factor in enhancing emission current density. 3-D emitter on $\mathrm{Cu}$-substrate registered a huge 23 times increment in emission current density over similar 3-D structure on alumina substrate. The results found during the present study clearly indicate that a 3-D emitter 
structure on a conductive substrate (such as $\mathrm{Cu}$ ) offers much higher efficiency, as compared to conventional 2-D emitters on metallic substrates or other 3-D emitter structures on alumina substrates.

\subsection{Structure and Field Emission Performance of Transparent Flexible Field Emission Device}

After successful demonstration of energy efficient field emission devices (in terms of low turn-on field and high emission current) through application of interface engineering and 3-D design, the next was to incorporate two more exciting features to field emitters - flexibility and transparency. Application of these flexible and transparent displays ranges from flexible head-up displays, foldable electronics, lightning tiles and others. A hybrid structure of 1-D CNTs over 2-D graphene can be an ideal architecture for application as flexible and transparent field emission displays.

Graphene was synthesized through CVD process on $\mathrm{Cu}$ foils and transferred onto polyethylene terephthalate (PET) substrate using hot press lamination and chemical etching process. Details about graphene synthesis and transfer processes were given in section 3.2.3, and basic properties of the transferred film are given in appendix-4. Figure 4.23 shows the schematic processing chart of field emitter device fabrication. For fabricating the hybrid graphene/CNT cathode, multiwall carbon nanotube (MWCNT) solution was spin coated over graphene/PET substrate. MWCNT solution was prepared in polyvinyl alcohol (PVA) and de-ionized (DI) water mixture. Anode was made by dip coating the green phosphor over the graphene/PET film. Anode and cathode were stacked over each other for fabricating flexible, transparent field emission device. 


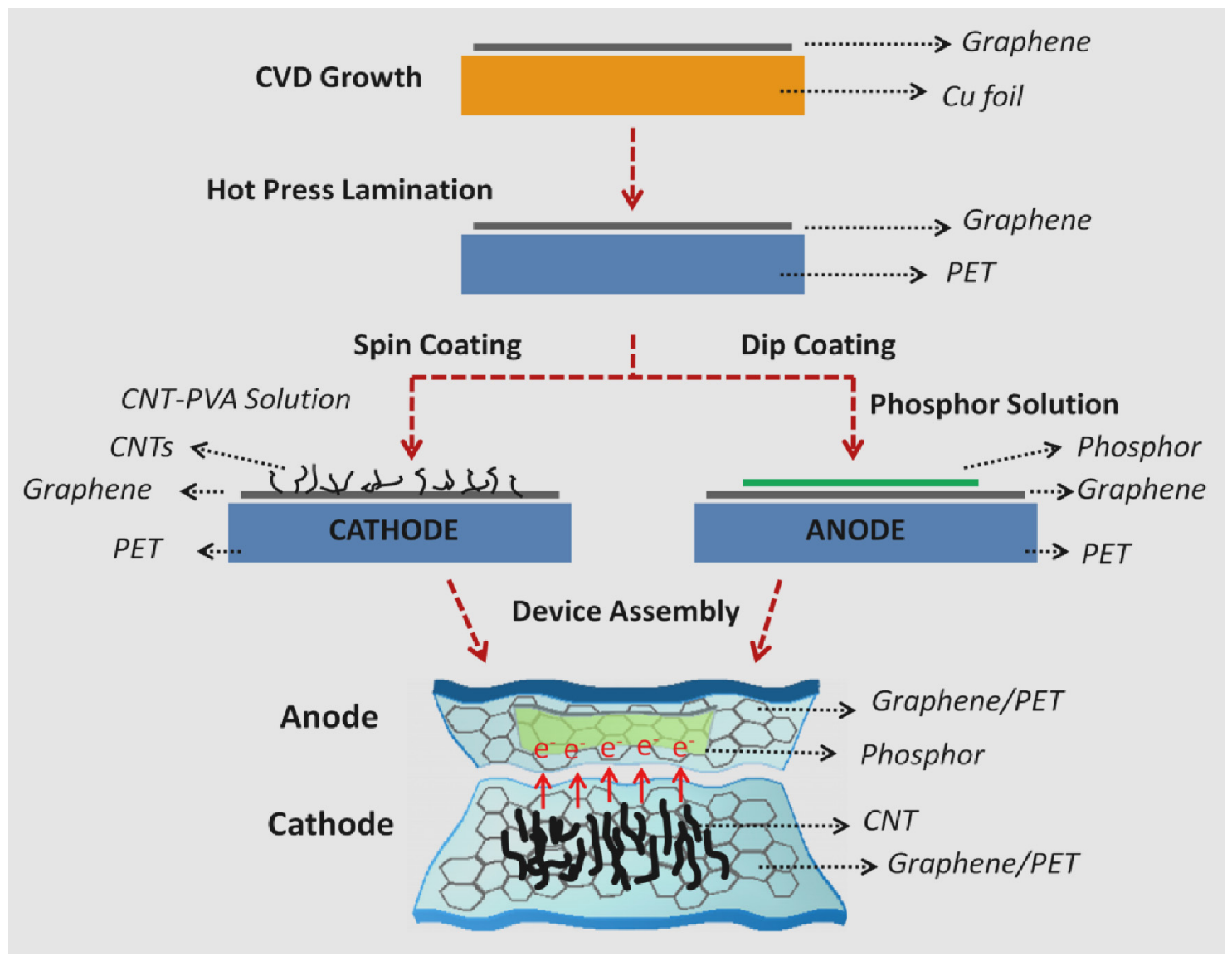

Figure 4.23: Process flow for cathode and anode preparation. Schematic of assembled full flexible and transparent FE device showing CNT-graphene/PET cathode and phosphor coated graphene/PET anode.

Figure 4.24 shows a representative SEM image of PVA coated MWCNT emitters on graphene/PET. The average diameter and length of MWCNTs were $60 \mathrm{~nm}$ and 1-3 $\mu \mathrm{m}$, respectively. Many CNTs were found to be in erected position, which was suitable for good field emission response. PVA acted as binder for MWCNT on graphene film and provided stability to the emitter under strong electric field [108-110]. A low density $\left(\sim 1.2 \times 10^{8} / \mathrm{cm}^{2}\right)$ of CNTs over graphene film was maintained for high optical transparency of cathode. 


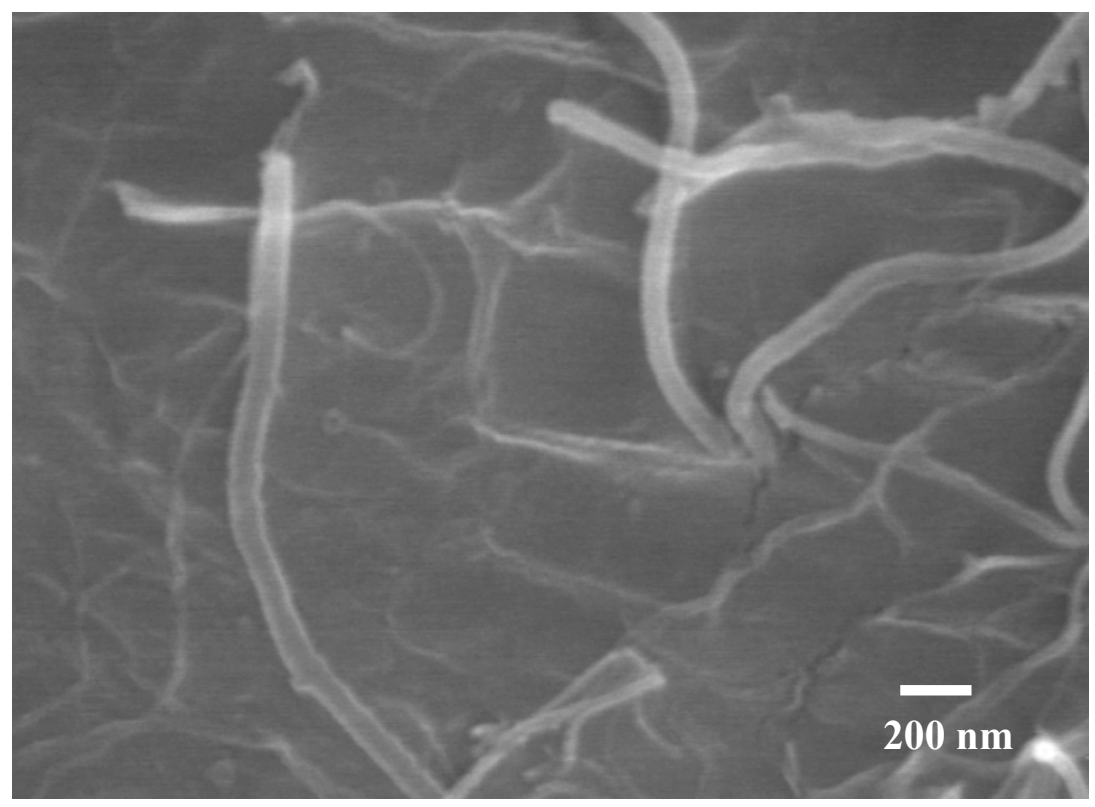

Figure 4.24: Structure of the graphene-CNT hybrid emitter - SEM image of PVA coated CNTs on graphene/PET film.

Both the cathode (MWCNT/graphene/PET) and the anode (phosphor/graphene/PET) were framed with adhesive conductive copper tape at the edges (figure 4.25 a). Field emission device was assembled over a glass tube (27 mm diameter) by stacking the electrodes over each other, using $\sim 600 \mu \mathrm{m}$ thick spacers along their edges. Such an assembly aids to quantify field emission response of the device in a bent state. Figure 4.25 (b) shows a photo of the assembled fully transparent and flexible field emission device (dimension $15 \mathrm{~mm} \times 15 \mathrm{~mm}$ ). This image clearly shows the flexibility and transparency of our device. While its ability to perfectly take the contour of the glass tube proves its flexibility, clearly visible letters through this device supports the claim of high transparency. Slight difference in the visibility of the letters was mainly due to the 
presence of thin film of phosphor coating on the anode. Inset of the figure shows the field emission image captured from the anode end of the device.
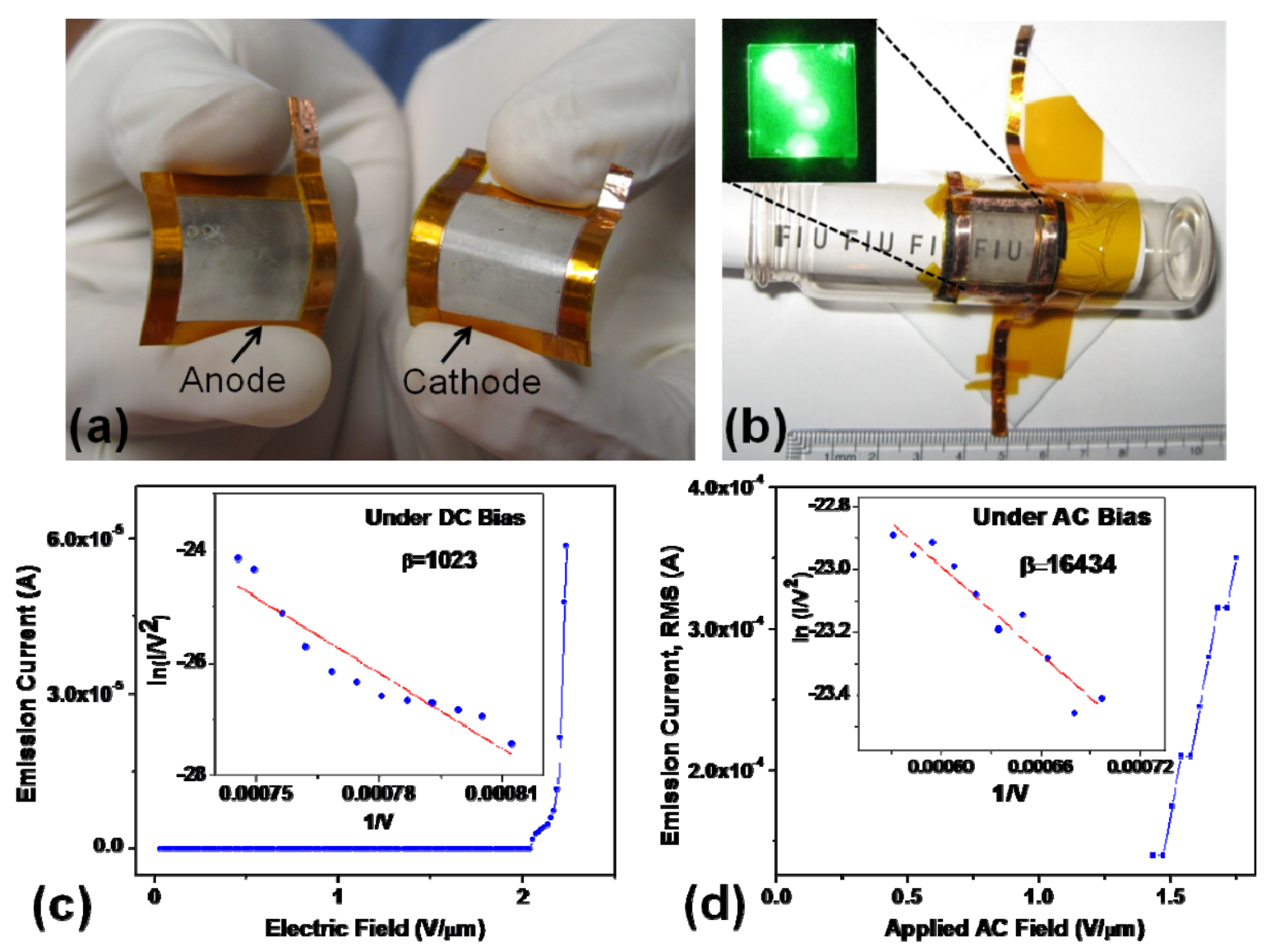

Figure 4.25: (a) Images of flexible and transparent anode and cathode. (b) Fully transparent and flexible FE device assembled on glass tube ("FIU" character can be seen from the front side of the FE device). Inset shows FE image from the device. (c) Emission current versus electric field characteristics of FE device operating under DC bias voltage. Inset shows corresponding F-N plot. (d) Emission current versus electric field characteristics of FE device operating under AC bias voltage. Inset shows corresponding F-N plot. 
Field emission response of the device is presented in figure 4.25 (c) - (d). Emission current as a function of applied DC bias to the device is shown in figure 4.25 (c). Turn-on field ( $\mathrm{E}_{\mathrm{TO}}$, defined as the electric field to generate $1 \mu \mathrm{A} / \mathrm{cm}^{2}$ current density) of the device was found to be $2.05 \mathrm{~V} / \mu \mathrm{m}$. Low turn-on field from a FED ensures that the device started performing at a very low applied field and hence, was always desirable. In fact, the present result showed much lower turn-on field, as compared to the flexible, transparent cathode structure, reported very recently (33\% of the value mentioned in that reference) [111]. Similarly, the threshold field ( $\mathrm{E}_{\mathrm{Th}}$, defined as the electric field required to generate $10 \mu \mathrm{A} / \mathrm{cm}^{2}$ current density) was also found to be extremely low, $2.2 \mathrm{~V} / \mu \mathrm{m}$, which is almost $20 \%$ of the comparable value in ref. [111]. It may be mentioned here that unlike ref. [111], which presented only a flexible and transparent C-nanostructure based cathode for application in FEDs, our device allowed flexibility and transparency to both the cathode and anode. The images on the back side of the screen can be seen through the all-graphene based display as seen in figure 4.25 (b), thus the assembled device can be used for transparent and flexible display.

In order to calculate the field enhancement factor $(\beta)$, Fowler-Nordheim (F-N) equation was used and the corresponding plot is included as insets of figure 4.25 (c) and (d). F-N equation is stated as $\mathrm{I}=\left(\mathrm{aA} \beta^{2} \mathrm{E}^{2} / \Phi\right) \exp \left(-\mathrm{b} \Phi^{3 / 2} / \beta \mathrm{E}\right)$, where $\mathrm{I}$ is the emission current, $\mathrm{A}$ is emission area, $\beta$ is the field enhancement factor, $\mathrm{V}$ is applied voltage, $\varphi$ is the work function of the emitter (assuming work function of CNTs to be similar to graphite, i.e., $5.0 \mathrm{eV}$ ), a and $\mathrm{b}$ are two constants, of values $1.54 \times 10^{-6} \mathrm{~A} \mathrm{eV} / \mathrm{V}^{2}$ and $6.83 \times$ $10^{7} \mathrm{eV}^{3 / 2} \mathrm{~V} / \mathrm{cm}$, respectively. From the F-N plot, field enhancement factor has been 
calculated as 1023, in DC bias. The straight line nature of the F-N plot ensures that the emission process was through tunneling of electrons from CNTs.

Further, the FED was subjected to AC bias, using a $1 \mathrm{kHz}$ sine-wave function and the emission response is presented in Figure 4.25 (d). In AC bias, the turn-on field further reduces down to $1.4 \mathrm{~V} / \mu \mathrm{m}$ and a total emission current of $350 \mu \mathrm{A}$ could be achieved. Moreover, under AC bias, the field enhancement factor is highly enhanced to a value of 16434. Emission behavior of this graphene based field emitter seems to be impressive. It may be noted here that in this design of the device, graphene (on flexible, transparent polymer) mainly acted as the highly conductive base material, while CNTs embedded onto the graphene performed as the actual electron emitting sources. Figure 4.26 shows stability of the emission current under DC bias (at an electric field of $2.15 \mathrm{~V} / \mu \mathrm{m}$ ), for a continuous operation of 10 hours. Variation in current was found to be low and the device offered very good stability over the experimental time-period.

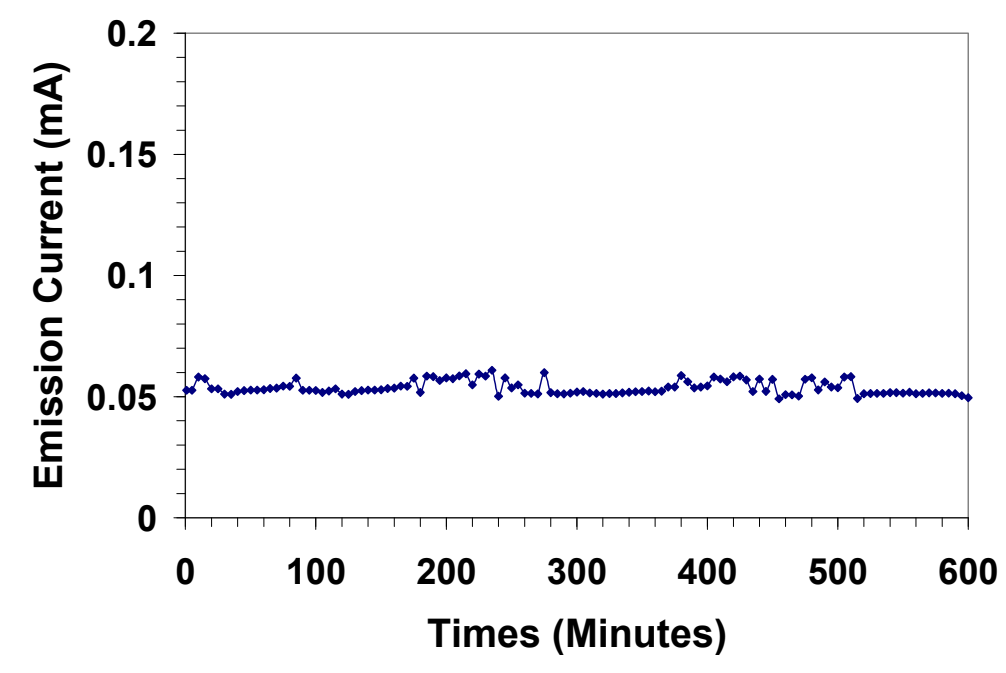

Figure 4.26: Emission stability of the graphene-CNT hybrid field emitter, under DC bias. 
Overall, the results presented here show that the CNT-graphene hybrid structure based field emitter device is also highly energy efficient and has good potential for flexible and transparent field emission devices.

\subsection{Structure and Performance of Carbon Nanotube Based Anode in Li-ion Battery}

In the previous sections, successful research efforts to manufacture energy efficient carbon nanotube based field emitters have been presented. After field emitter, the focus was on application of CNTs in energy storage devices, such as Li-ion batteries. Application of CNTs in Li-ion batteries is not new [112-114], though the results were not encouraging. CNT-based Li-ion batteries have offered lower specific capacity of the battery, as compared to the conventional graphite anode based Li-ion batteries. Analyzing the failures, it was observed that CNT was added in a small percentage (to the electrode) along with other active materials like graphite, active carbon etc. and organic binders. These materials were mixed together as a paste and painted on current collector materials like copper, aluminum or stainless steel. This process of anode preparation did not allow direct contact between CNTs, electrolyte and $\mathrm{Li}^{+}$ions and hence, effect of CNT addition could not be fully understood.

To address all these issues, the present research aimed at direct synthesis of interface-controlled MWCNTs on copper current collectors and their application as the anode in Li-ion cells. This kind of structure is expected to have many advantages over the conventional anodes. First of all, unlike all the past studies involving CNTs, which used raw CNTs and polymeric binders [112-114], the present structure would have directly 
grown CNTs on the current collector, thus avoiding the polymeric binders completely. In this way, it will reduce harmful effect of the polymeric binder, reduce weight of the active material, increase specific capacity and show potential to be used for high temperature application. Secondly, CNTs do not have any kind of expansion/contraction and pulverization problem (like $\mathrm{Si}$ and $\mathrm{SnO}_{2}$ ), so it will sustain its capacity for long cycles. Third, growth of CNTs on interface-engineered metallic substrates will ensure that each CNT is well bonded to the current collector, thus all of them contribute to the capacity. Fourth, high specific surface area of CNTs will allow more Li-ion intercalation. Fifth, higher conductivity of the active anode material is important for achieving higher capacity [115]. In that respect, MWCNTs, known to be excellent charge carriers, are an alternative option and aid in achieving higher capacity. Moreover, by the interfacecontrol, proposed in this study, an ohmic contact and strong bonding between the CNTs and substrate will be ensured, which will further help in efficient charge transport. Further, the anode structure of MWCNTs grown on $\mathrm{Cu}$ foil, can be very easily fabricated using thermal chemical vapor deposition (CVD) process. Keeping in view of all these multifarious advantages, the present study used interface-controlled MWCNTs grown on $\mathrm{Cu}$ current collector as the anode for Li-ion batteries. This novel anode structure was tested as half-cell (as described in sections 3.3 and 3.6) and results are discussed below.

The novel anode structure was prepared by depositing catalysts (through sputtering) onto $\mathrm{Cu}$ foils, followed by MWCNT growth by CVD. Electrochemical studies are performed on half cells prepared by CR2032 press. Electrochemical characteristics of the MWCNT-on-Cu electrode are presented in figure 4.27. First two charge-discharge curves, at a current rate of $0.1 \mathrm{C}\left(38 \mathrm{mAg}^{-1}\right.$, assuming theoretical specific capacity same as 
that of graphite, i.e. $372 \mathrm{mAhg}^{-1}$ ), are shown in figure 4.27a. The first discharge cycle wais characterized by very high specific capacity of $2547 \mathrm{mAhg}^{-1}$, while the following de-lithiation (charge) cycle showed specific capacity of $1455 \mathrm{mAhg}^{-1}$. This led to an irreversible capacity loss of $42 \%$. However, the second discharge and charge cycles almost maintained the reversible capacity from the first cycle. During the first discharge cycle, a plateau in the voltage region $0.9-0.5 \mathrm{~V}$ was observed. Such plateau is typical of all CNT-based electrodes and is related to decomposition of the electrolyte and formation of solid electrolyte interphase (SEI) on the CNT surface [116]. The plateau was not observed from the next cycle onwards, indicating that SEI formation was complete in the first discharge cycle itself. A good fraction of the capacity was found to be at a voltage range $>0.5 \mathrm{~V}$, which was a common feature for all nanostructured carbon anode materials and even for C-Si core-shell nanowires [117-120]. Though this type of behavior was different from that shown by graphite electrodes [121], it did not affect the full-cell characteristics much, as was shown for C-Si core-shell nanowire structure [118].

Rate capability of the electrode is shown in figure 4.27 (b). Reversible capacity of the electrode was found to be very high and quite stable (especially, after few initial cycles). Very high specific capacity could be observed at all current rates. Even at very high charging/discharging rate of $1.116 \mathrm{Ag}^{-1}$ (3.0C), the electrode showed a reversible capacity of $767 \mathrm{mAhg}^{-1}$, representing $106 \%$ increment in capacity as compared to the theoretical capacity of graphite anodes $\left(372 \mathrm{mAhg}^{-1}\right)$. This huge increment in specific capacity, that too in a higher current rate, proves suitability this anode structure for possible application in Li-ion batteries. 

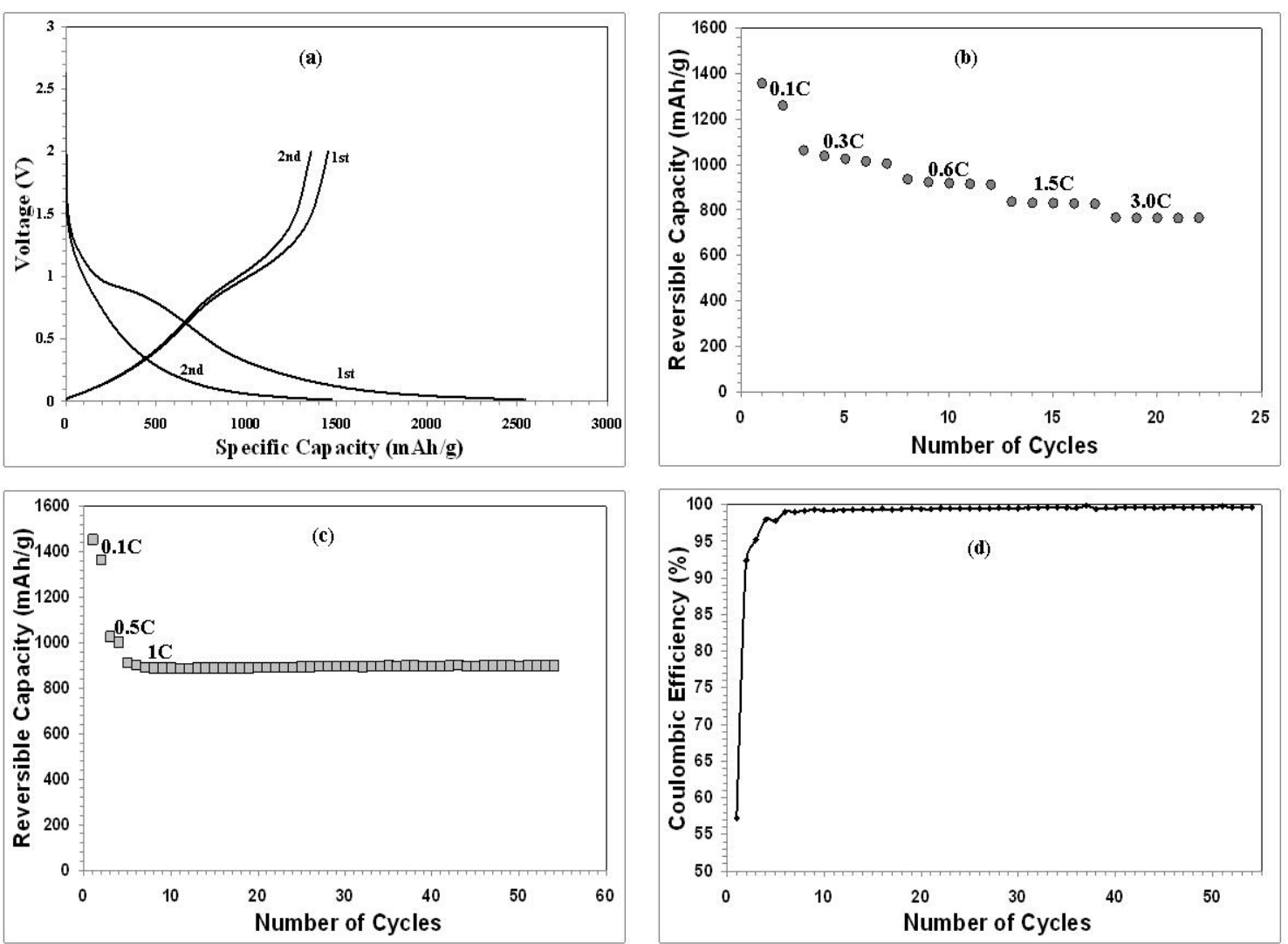

Figure 4.27: Electrochemical characteristics of the proposed CNT-based electrode structure. (a) First two charge-discharge cycles of the MWNT-on-Cu anode, at $38 \mathrm{mAg}^{-1}$ rate. (b) Reversible capacity of the MWNT-on-Cu anode, at different C-rates. (c) Exceptional stability of the reversible capacity $\left(\sim 900 \mathrm{mAhg}^{-1}\right)$ of the MWNT-on-Cu anode in long-run, at $372 \mathrm{mAg}^{-1}$ rate. (d) Coulombic efficiency of the MWNT-on-Cu anode, showing very high efficiency, except for the first cycle. After initial 5 cycles, the efficiency remained more than $99 \%$.

To specifically understand the stability of capacity, the electrode was subjected to 50 charge/discharge cycles at $372 \mathrm{mAg}^{-1}$ rate (1.0C), after two initial cycles each in $0.1 \mathrm{C}$ $\left(38 \mathrm{mAg}^{-1}\right)$ and $0.5 \mathrm{C}\left(186 \mathrm{mAg}^{-1}\right)$ rate (figure $\left.4.27 \mathrm{c}\right)$. The figure shows very high capacity of the electrode $\left(\sim 900 \mathrm{mAhg}^{-1}, 140 \%\right.$ enhancement as compared to theoretical 
capacity of graphite) at this rate and excellent stability of the capacity over 50 cycles. In fact, there was nil capacity degradation during these 50 cycles (except for the initial two cycles). The coulombic efficiency of the electrode was also very high, more than $99 \%$, after two initial cycles at $38 \mathrm{mAg}^{-1}$ rate (figure $4.27 \mathrm{~d}$ ). Such high capacity and nil capacity degradation over 50 cycles make this electrode a suitable alternative to the graphite anodes, conventionally used in Li-ion batteries.

It is well understood that the unique structure of the proposed anode is responsible for its excellent performance. In order to better understand the structure and lithiationdelithiation mechanism, a thorough structural characterization, using SEM, high resolution transmission electron microscope (HRTEM), X-ray diffraction and Raman spectroscopic analysis has been performed. X-ray diffraction (figure 4.28 a) and Raman spectroscopy analysis (figure 4.28 b) showed different peak shapes and intensity ratios in different stages, pointing towards a possible structural change.

Figure 4.28 (a) shows XRD patterns of the proposed anode structure in the pristine, lithiated and delithiated stages, respectively. Due to the presence of high intensity peaks from $\mathrm{Cu}$ substrate, no other peaks, except $\mathrm{C}(002)$, could be pointed out in the XRD pattern. Broadening of the $\mathrm{C}(002)$ peak, in lithiated condition, indicates possibility of increase in defect concentration in the samples. However, the broadening was not observed in the delithiated sample. A semi-quantitative analysis, using CMPR software showed that the full width at half maxima (FWHM) of the peaks increased from as-grown state $\left(\mathrm{FWHM}=2.20^{\circ}\right)$ to lithiated state $\left(\mathrm{FWHM}=4.81^{\circ}\right)$ and then decreases upon de-lithiation $\left(\mathrm{FWHM}=2.75^{\circ}\right.$ ). Since, FWHM can be related to the defect density in 
the material, it can be concluded that lithiation increased defect sites on the CNTs and delithiation eliminated most of these defects.
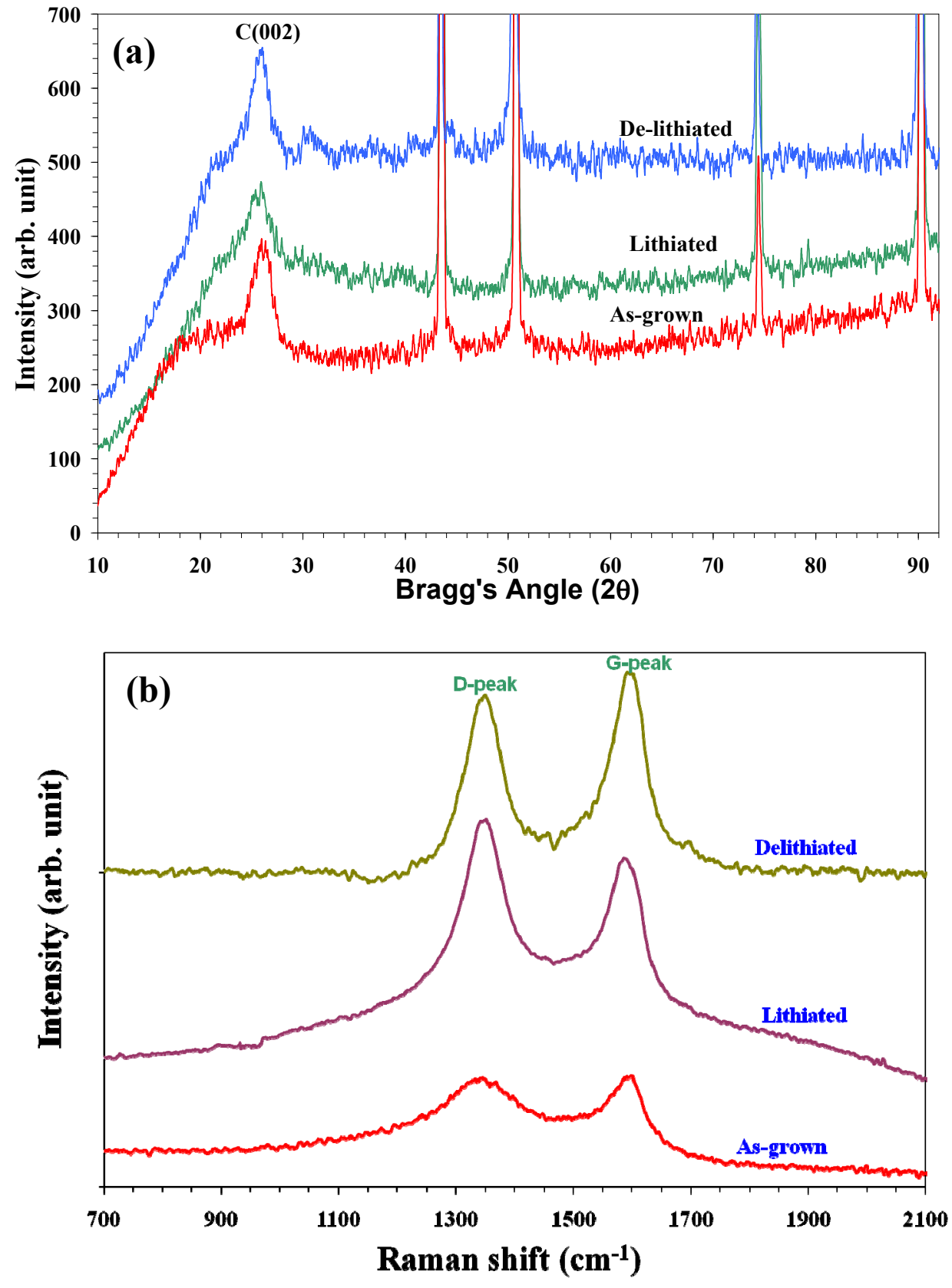

Figure 4.28: Structural comparison of the proposed anode structure in different stages. (a) X-ray diffraction patterns and (b) Raman spectroscopic analysis. 
The observations made from XRD match well with Raman spectroscopic analysis. $\mathrm{I}_{\mathrm{D}} / \mathrm{I}_{\mathrm{G}}$ ratio in Raman spectra, which indicates purity or crystallinity of $C N T \mathrm{~s}$, increased from 0.95 for the pristine sample to 1.21 in the lithiated condition and decreased to 0.94 in the delithiated condition. So, the crystallinity of the CNTs was lost in lithiated state and recovered in delithiation stage.

Figure 4.29 (a) (and its inset) shows SEM micrograph of the as-grown CNTs on the $\mathrm{Cu}$ current collector, while figure 4.29 (b) (and its inset) shows TEM micrographs of the same structure. The CNTs (outer diameter $\sim 100 \mathrm{~nm}$ ), which formed a forest-like structure of $\sim 30 \mu \mathrm{m}$ height on the $\mathrm{Cu}$ current collector, created a porous network and opened up huge surface area of CNTs, thus increasing the lithiation capability of the electrode. The as-grown CNTs also showed some amount of defects in the structure (inset of figure $4.29 \mathrm{~b}$ ), which could probably lead to enhanced Li-ion intercalation. Some of the CNTs were found to be twisted (figure $4.29 \mathrm{c}$ ). The twisting increased specific surface area of CNTs, which was probably one of the reasons of high Li insertion in these CNTs.

No noticeable volume expansion of the CNTs was observed after lithiation. Figure 4.29 (b), (d) and (e) shows representative SEM micrograpghs of CNTs in asgrown, lithiated and delithiated states indicating that the diameter of CNTs remained almost constant $(\sim 100 \mathrm{~nm})$. This observation is unique and distinctly different from the observations made by Maurin et al. [122], who has reported swelling and shrinkage of CNTs, during the lithiation and delithiation. Absence of this volume change in the CNT anode is beneficial for practical application. 

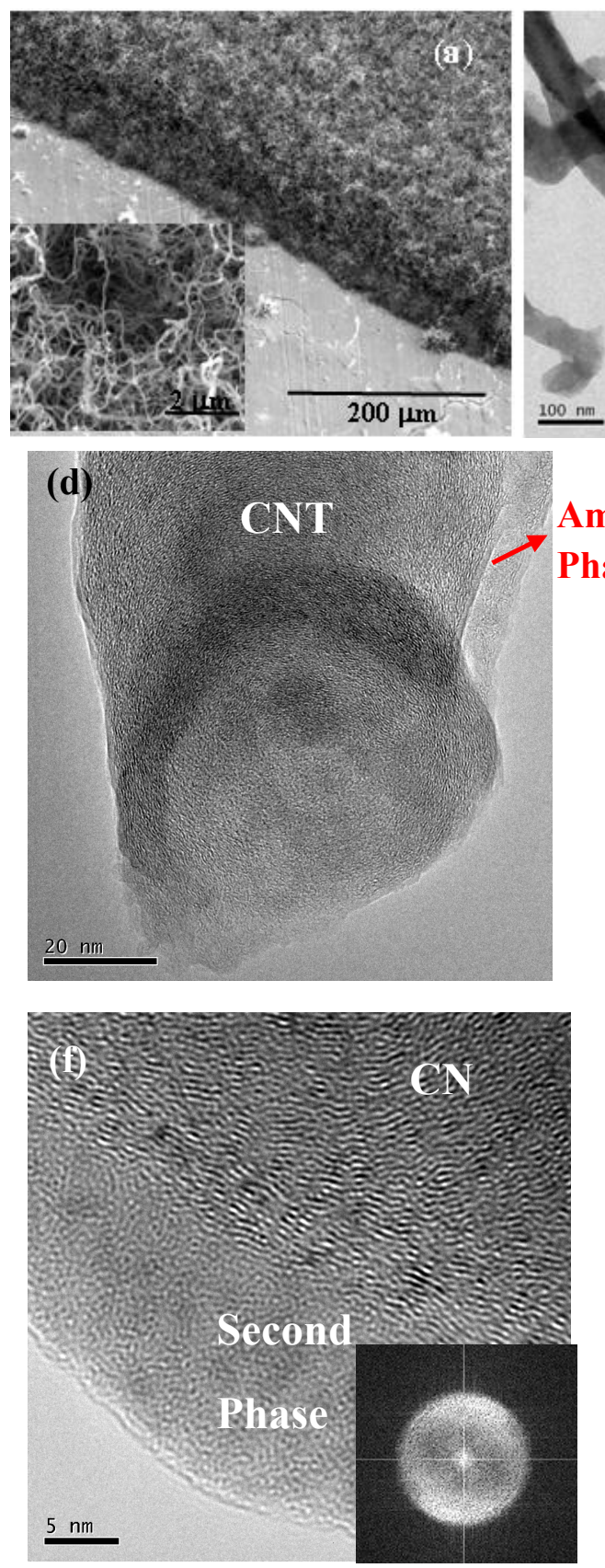

(b)

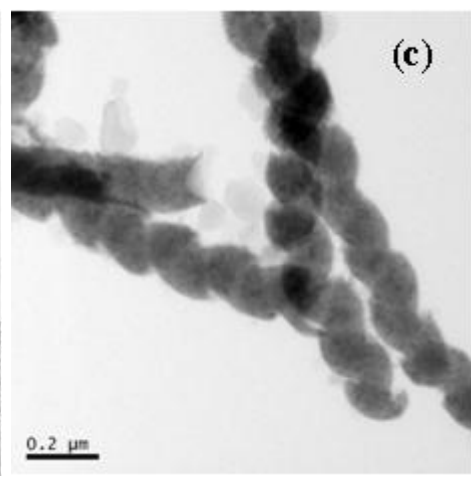
Amorphous Phase

\section{(e)}
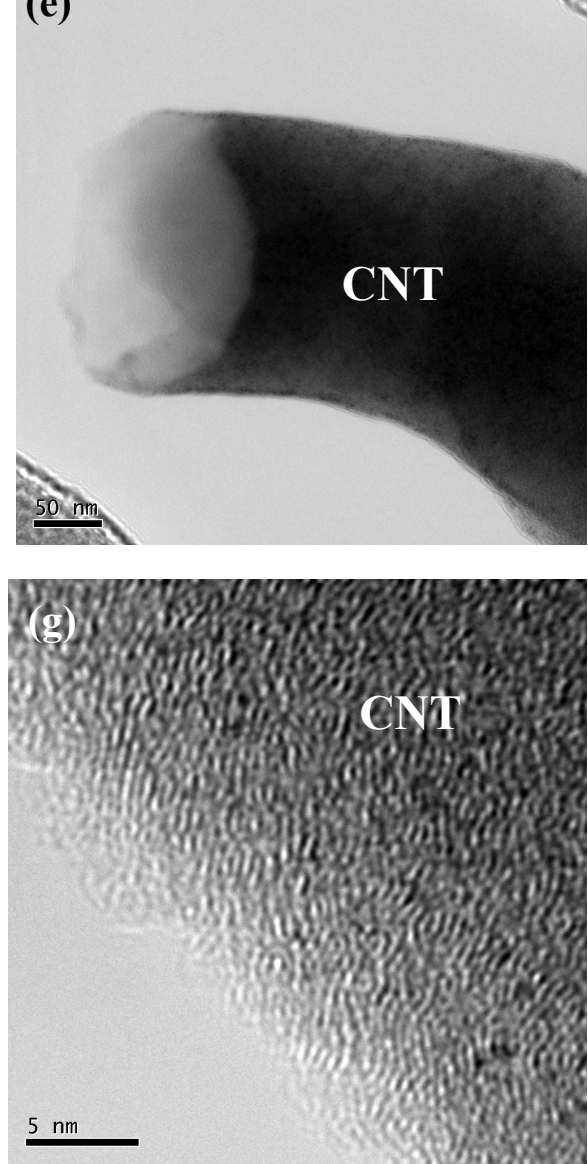

Figure 4.29: Structure of the electrode in as-grown, lithiated and delithiated states. (a) SEM and (b) TEM images of the as-grown MWNT-on-Cu structure (insets are at higher magnification). (c) TEM images of twisting in the as-grown sample. (d) and (f) show TEM images of the CNTs in lithiated condition (FFT image at inset). (e) and (g) are TEM images of the CNTs in delithiated condition, showing absence of the second phase. 
A quick comparison of the structure of the CNTs, in lithiated and delithiated can be performed from the TEM images in figure 4.29 (d) - (g). The lithiated CNT structures clearly showed formation of thick layer of a second phase (the lithiated phase) on their walls, while such a second phase structure could not be observed in delithiated condition. This second phase could be Li-oxide phase, which might have formed during mishandling of the sample during TEM sample preparation or it could also be a second phase (Li-C) formed during lithiation. To minimize exposure of sample to air, the sample preparation was carried out in Ar glove box. Further, to confirm the effect of long-time air exposure on the structure, a separate study has been performed. Figure 4.30 (next page) shows the outcome of this study, in terms of TEM and HRTEM images. For the comparison of amorphous lithiated carbon and lithium oxide, delithiated sample was exposed to air for long time and has been investigated. Even though most regions of CNT wall were recovered and crystallized, fine nanocrystals were observed at few junctions of CNTs. The HRTEM and its corresponding FFT, as shown in the figure, clearly reveals the existence of crystalline phase having 4 fold symmetry with $2.3 \AA \mathrm{d}$-spacing, which corresponds to the (002) plane of cubic- $\mathrm{Li}_{2} \mathrm{O}$ phase. The structure of the observed oxides which are commonly observed after charge and discharge experiment in Li-ion battery, was identified as face centered cubic $\mathrm{Li}_{2} \mathrm{O}$ with $\mathrm{a}=4.6 \AA$. Dupont et al. [123] reported $\mathrm{Li}_{2} \mathrm{O}$ structure using in-situ XRD, coupled with carefully designed HRTEM experiment. Our results were in close agreement with their reported values. The inverse FFT image using the indicated arrows in filtered FFT, as shown in (d), clearly demonstrates the crystalline phase on the CNT surface. 

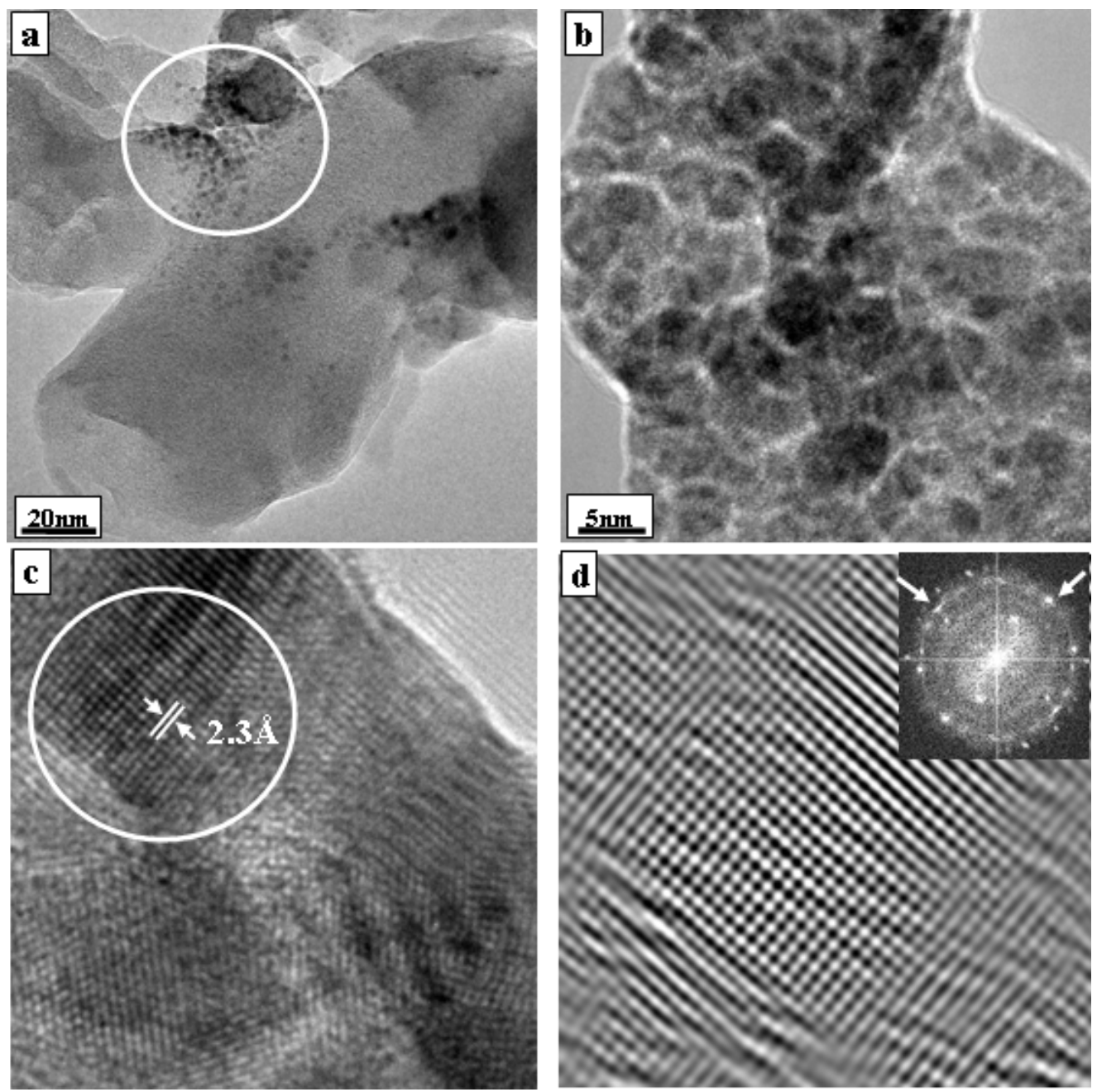

Figure 4.30: (a) TEM image of MWCNTs showing fine nanocrytalline particles on the MWCNTs surface, (b) higher magnification of circled region shown in (a), (c) HRTEM image on the one of the nanocrystalline particle showing the lattice fringes, (d) Inverse FFT image indicated by arrow from the filtered FFT showing 4-fold symmetry of reciprocal lattice fringes.

A quick comparison of the $\mathrm{Li}_{2} \mathrm{O}$ phase and the second phase formed in figure 4.29 (f) immediately points out that the latter is not the oxide. So, thick layer on the lithiated CNTs was expected to be an amorphous lithiated carbon phase only. Formation of such 
structures was found throughout the whole lithiated sample. This amorphous lithiated phase was expected to have a stoichiometry of $\mathrm{LiC}_{\mathrm{x}},(\mathrm{x}-2-6)$, as reported previously for C-based anodes of Li-ion batteries [124]. Though the specific stoichiometry of the lithiated compound was not known, very high defect density of CNTs and high reversible capacity $\left(>900 \mathrm{mAhg}^{-1}\right)$, which was comparable to the theoretical capacity (1116 $\mathrm{mAhg}^{-}$ ${ }^{1}$ ) of SWCNTs (forming $\mathrm{LiC}_{2}$ ) [124, 125], indicates that the composition is likely to be $\mathrm{LiC}_{\mathrm{x}}(\mathrm{x}=2-3)$. However, the composition needs to be confirmed by Li nuclear magnetic resonance (NMR) technique. These results clearly indicate that during lithiation CNTs partly lose their crystallinity along the outer surface, probably due to their interaction with the $\mathrm{Li}^{+}$ions. However, the same samples in de-lithiated state did not show presence of the thick amorphous phase on the walls of CNTs (see Figure 4.29 e, g). It was not very clear, if the loss of crystallinity was fully recovered during delithiation process, but the HRTEM images of the delithiated sample exhibiedt better crystallinity, demonstrating at least a partial recovery of loss of crystallinity.

Structural characterization of the samples, in different stages of lithiation, shaded light on the mechanism of lithium insertion and extraction into the anode and highlighted the advantages of the anode structure. Initial higher defect density of the pristine CNTs, as evident from the Raman spectroscopic analysis, XRD and HRTEM, might have helped the anode structure to show very high capacity, as defective CNT structure is known to have higher conductivity than graphitic CNTs [126] and better lithiation capability [117]. Moreover, twisted CNTs offered higher specific surface area and hence, probably more Li-ion intercalation. During lithiation, $\mathrm{Li}^{+}$ions reached individual CNTs, passing easily through the CNT forest structure and attached with their sidewalls. Very high surface 
area of the CNTs promoted huge amount of $\mathrm{Li}^{+}$ion intercalation. On the other hand, during delithiation, most of the ions returned back to the opposite electrode. Highly porous nature of the $\mathrm{CNT}$ forest structure allowed easy transport of the intercalating ions from one electrode to the other. A schematic of the lithiation-delithiation mechanism is given in figure 4.31 .

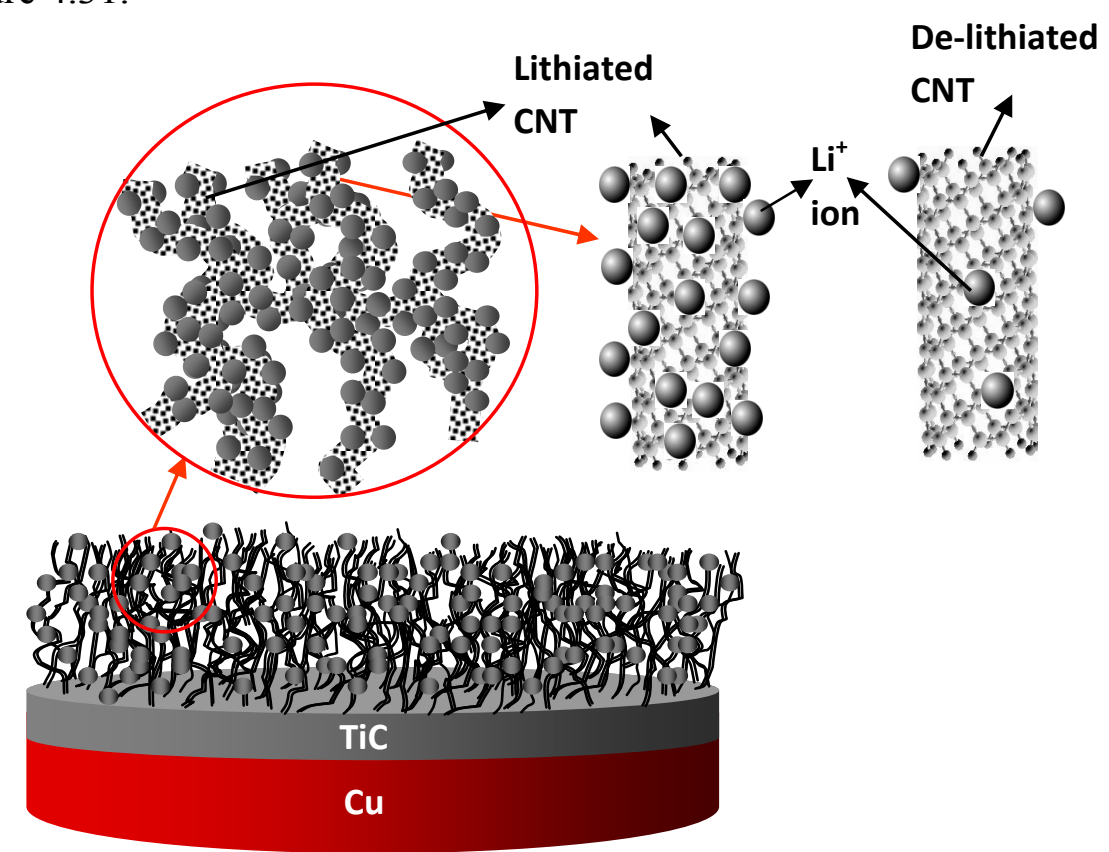

Figure 4.31: A schematic (not to scale) of the proposed lithiation-delithiation mechanism, showing huge amount of $\mathrm{Li}^{+}$ion intercalated to walls of CNTs during lithiation and de-intercalation of most of the ions in delithiation.

It is very important for the Li-ion battery to have an efficient electron transport from the current collectors to the CNTs to show good cycling behavior. Interfacecontrolled MWCNT structure, grown directly on $\mathrm{Cu}$ current collector, ensured minimum resistance. Through the choice of diffusion barrier layer and catalyst layer ( $\mathrm{Ti}$ and $\mathrm{Ni}$, respectively), presence of high-resistivity material in the electron path has been 
minimized. Total resistance of the electrode structure (Cu to CNT tip), as measured by a two-probe method, was found to be $3.3 \Omega$, which wais considered to be very low. It may be mentioned here that such kind of contact resistance measurements between substrate and CNT tip resulted in lowest value of $\sim 135 \Omega$ [127], which is almost 40 times higher than the values found in the present study. One of the reasons to achieve such low contact resistance is presence of $\mathrm{Ti}$, which is known to show very low (an order of magnitude lower than that of $\mathrm{Pd}, \mathrm{Pt}, \mathrm{Cu}$ and $\mathrm{Au}$ ) contact resistance with CNTs [128]. Moreover, it was necessary to have good bonding between substrate and CNTs, in order to avoid pulverization and to achieve good stability in high-cycle. Strong bond formation between CNTs and $\mathrm{Cu}$ substrate, through Ti interfacial layer, has already been shown in section 4.2. HRTEM image, taken from the interface region of the pristine sample, showed very good bonding between the substrate and the CNT layer, through formation of an interface layer (figure 4.32).
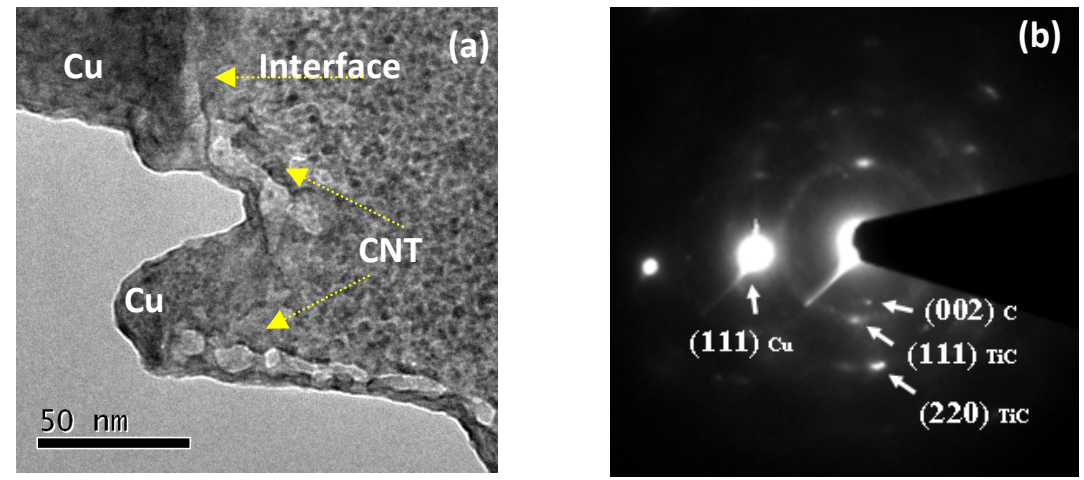

Figure 4.32: Structural analysis of the proposed anode. (a) HRTEM image of substrateCNT interface, showing well-bonded interface and presence of interfacial phase. (b) Selective area diffraction pattern showing formation of TiC at the interface. 
A selective area diffraction pattern (SADP) taken from this interface region showed presence of TiC. Formation of TiC, in the interface, helped in two ways: first, TiC, being highly conductive, provided low-resistance paths for electron movement from $\mathrm{Cu}$ current collector to CNTs and secondly, it ensured a strong bond formation between the substrate and the CNT [129].

The benefits of using an interface-controlled MWCNT structure, directly grown on $\mathrm{Cu}$ current collector, as the anode material in $\mathrm{Li}$-ion batteries can be appreciated from the schematic presented in figure 4.33 .

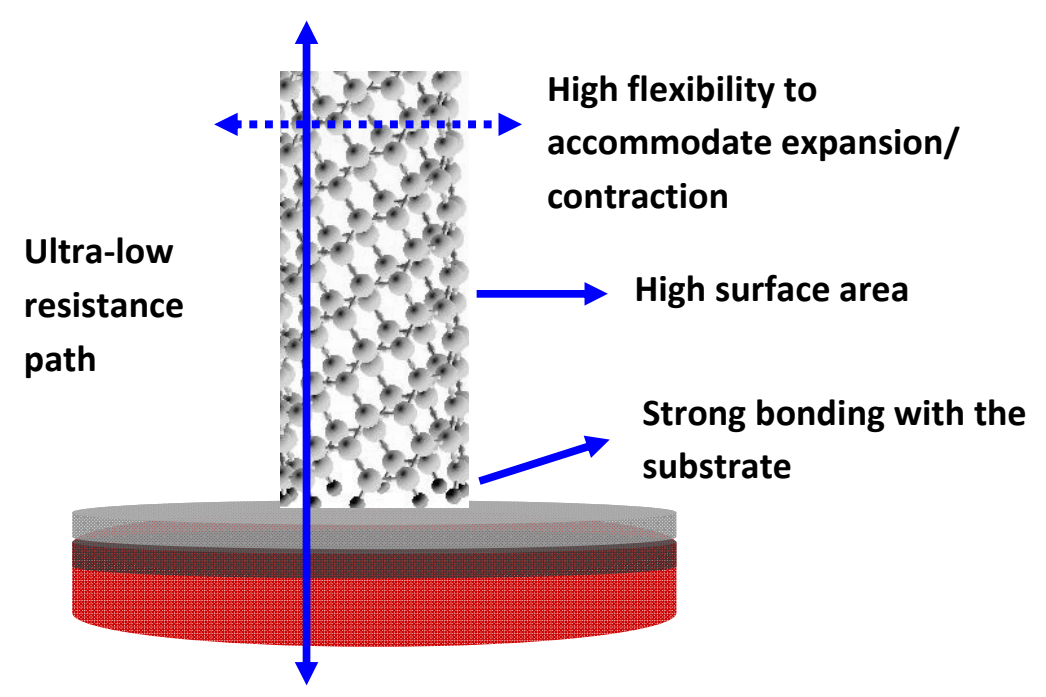

Figure 4.33: A schematic (not to scale) of the proposed anode structure, showing its advantageous features.

As shown in the drawing, this proposed structure offers many advantages: (i) CNT structure did not show any expansion/contraction problem during lithiation /delithiation and hence, posed no threat of pulverization; (ii) very high surface area of CNTs was available for lithiation and easy ion transport through the highly porous CNT 
forest structure; (iii) formation of $\mathrm{TiC}$ allowed a strong bonding of CNTs with the substrate, thus minimizing breakage and improving the long-cycle behavior and (iv) from CNT tip to copper current collector, the structure presented an ultra-low resistance path (CNTs having highest current carrying capacity, $\sim 10^{9} \mathrm{~A} / \mathrm{cm}^{2}$ and substrate material also being highly conductive), which aided in faster charge transport. All these beneficial factors of the interface-controlled MWCNT structure on copper current collector aided in enhancement of the capacity of the electrode and provided excellent stability. The stability offered by this MWCNT based electrode showed comparable performance with Si nanostructure based electrodes $[130,131]$ and even better capacity of $900 \mathrm{mAhg}^{-1}$ at a current rates of $1 \mathrm{C}$ as compared to $\mathrm{C}-\mathrm{Si}$ core-shell nanowire(Figure $3 \mathrm{~d}$ of reference $[118])$.

Apart from its high-cycle stability, the interface-controlled MWCNT-on-Cu anode has shown higher capacity than any other anode fabricated by carbon nano/mesostructure and its composites, at all the current rates. Figure 4.34 presents a comparison plot of reversible specific capacities of different anode materials, as a function of current rate. It is clearly visible that apart from Si-nanostructures, MWCNT-on-Cu anode material has offered either equal or higher specific capacity. Difference between capacities of the anodes was more prominent in high current rate zone, where MWCNTon- $\mathrm{Cu}$ outperformed all other anode materials by huge margin. This comparison plot clearly shows the advantages offered by the MWCNT-on-Cu anode, over other conventional and new materials. Depending upon the performance of this anode, it may be concluded that $\mathrm{CNT}$ is going to one of the important candidate materials for future high-capacity Li-ion batteries, offering better safety, too. 


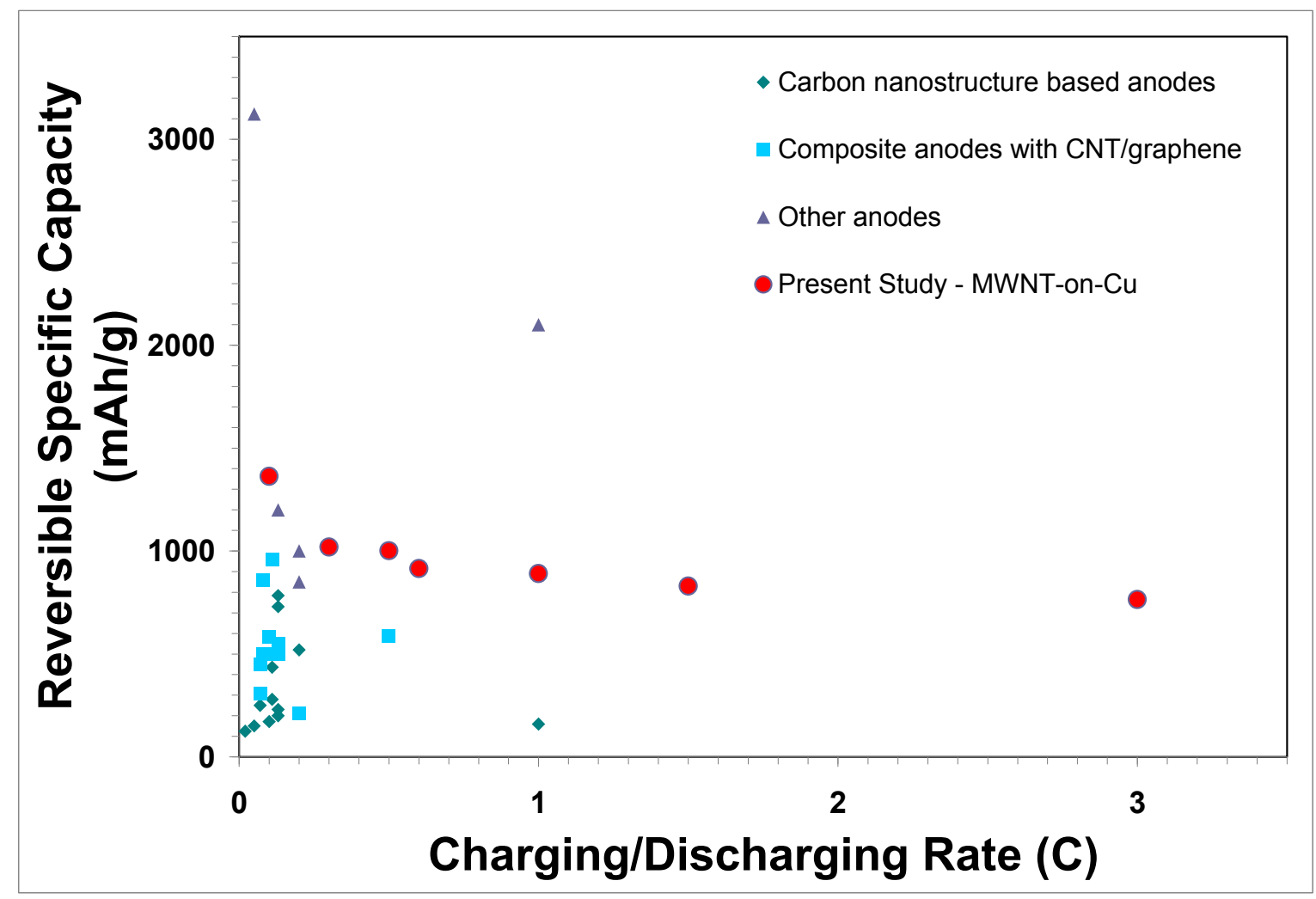

Figure 4.34: Comparison of reversible specific capacities of literature reported values of different types of anodes and that of the electrode used in present study, over a range of current rates. The interface-controlled MWCNT-on-Cu electrode has shown 1.5-5.5 times higher capacities than all reported carbon-based anodes [80, 112-114, 116, 117, 119, $120,125,132-143]$.

\subsection{Structure and Performance of Ultra-thin Alumina Coated Carbon Nanotube Based Anode in Li-ion Battery}

In an effort to further advance the benefits of MWCNT-on-Cu anode for Li-ion batteries, aim was fixed to enhance specific capacity of the electrode, with incorporation of more safety issues. Inactive metal oxides are often considered as an anode material or as one of the components of anode due to their ability to protect solid electrolyte 
interphase (SEI) and thus, contributing towards better cycle life and safety. Surface modifications of active anode materials, such as mild oxidation [144], coating by metal oxides [145, 146], have been suggested for higher efficiency electrodes. Among them, oxide-coated carbon nanostructures are expected to perform better, combining additional stability, safety and capacity offered by the oxide and excellent conductivity (thus, faster charge transport) and strength (rigidity of the structure) provided by C-nanostructures, specifically CNTs. Thus, a hybrid structure consisting of thin-layered oxide coated CNTs seems to be a promising candidate as an anode material for Li-ion batteries. A thin oxide coating on carbon nanotubes is expected to restrict unwanted reactions between carbon and the electrolyte and thus, provide extra stability to the anode [147]. It may be noted at this point that solid electrolyte interphase (SEI), which is known to be lithium ethylene di-carbonate [148], allows $\mathrm{Li}^{+}$ions to diffuse through it, while blocking the electrolyte molecules; ensuring reversible intercalation and de-intercalation of $\mathrm{Li}^{+}$ions during cycling [149]. Thus, stability of SEI is extremely important for good cyclability of Li-ion batteries. Surface modification by oxides was observed to improve bonding of the SEI with carbon, better wetting with electrolyte and less decomposition of SEI during chargedischarge [144-146], thus enhancing stability of the SEI. Improved stability of SEI also contributes towards better safety of the cell as SEI decomposition is known to be exothermic, leading to thermal runaway $[150,151]$. Recently, Y. Jung et al. have demonstrated that atomic layer deposition of alumina directly on natural graphite improved stability and safety performance of the Li-ion battery [147]. Enhanced performance, especially at elevated temperature, was attributed to formation of a protective surface layer on graphitic particles. Therefore, introducing a protective surface 
layer on nanostructured carbon materials is expected to further enhance its stability, while maintaining high capacity. In the present study, the prospect of a new anode consisting of an ultra-thin layer of aluminum-oxide coated on interface-controlled CNTs directly grown on $\mathrm{Cu}$ current collector was studied further.

During this study, atomic layer deposition (ALD) technique was applied to deposit alumina $\left(\mathrm{Al}_{2} \mathrm{O}_{3}\right)$. The novel anode was prepared by sputter deposition of catalysts onto the $\mathrm{Cu}$ foils, chemical vapor deposition (CVD) for direct CNT growth on the substrates and ALD to deposit $\mathrm{Al}_{2} \mathrm{O}_{3}$ on the randomly oriented CNTs. ALD is well established atomic layer coating process to generate conformal thin film structure on any convoluted network of high surface area material $[152,153]$. This approach to the development of the novel anode offers the obvious advantages of an oxide-CNT anode, i.e. good stability, enhanced safety and high capacity of alumina, along with faster charge transport, rigidity and capacity offered by the CNTs. This structure ensures extra safety by providing a binder-free nature of the cell and making a protective coating on the CNTs, thus shielding the CNTs and SEI from unwanted, exothermic reactions with the electrolyte. The results, as discussed below, have shown enhanced performance of the anode.

Details about CVD growth, anode preparation, button cell making, structural characterization and electrochemical characterization can be found in sections 3.1, 3.2.4, 3.3.2, 3.4 and 3.6, respectively. However, ALD was a new process step, which will be described here. During this process, remote plasma ALD (RPALD) was followed due to its ability to be performed at lower temperature $(523 \mathrm{~K})$ because of its reactivity of radicals and ions with precursor [154] and minimum damage of the substrate and the 
CNT structure, caused by the plasma process $[155,156]$. The process pressure and plasma power were kept at 0.5 Torr, and $100 \mathrm{~W}$, respectively. A trimethylaluminium (TMA, $\left.\mathrm{Al}\left(\mathrm{CH}_{3}\right)_{3}\right)$ as the $\mathrm{Al}$ precursor was introduced into the reactant chamber. $\mathrm{O}_{2}$ plasma was used as the oxygen reactant. Ar purge gas was introduced for complete separation of the precursor and $\mathrm{O}_{2}$ plasma.

The structure of the anode material is evident from figure 4.35. As described in the previous section, MWCNTs grown directly on the $\mathrm{Cu}$ current collectors, could be characterized as a thick forest of randomly oriented CNTs, having diameter in the range of 70-100 nm and $\sim 30 \mu \mathrm{m}$ long, some of them being twisted (which further increased the available surface area for $\mathrm{Li}^{+}$ion intercalation). Presence of huge free space around CNTs allowed formation of a porous network through which $\mathrm{Li}^{+}$ions could migrate easily and reached to all CNT surfaces. SEM and TEM images (figures 4.35 a-d) showed presence of a second phase on the CNTs. Figure 4.35 (c) shows presence of (113) of $\mathrm{Al}_{2} \mathrm{O}_{3}(\mathrm{~d}=$ $0.21 \mathrm{~nm}$, ICDD 2010 card no. 00-001-1243) and (002) of C (0.34 nm, ICDD 2010 card no. 00-001-0640). High angle angular dark field scanning transmission electron microscope (HAADF-STEM) image (figure 4.35 d), through Z-contrast, clearly shows presence of a different phase and localized elemental analysis (figure 4.35 e and f) shows presence of $\mathrm{Al}$ and $\mathrm{O}$ on different positions of walls of the CNTs. Figure 4.35 (g) presents HRTEM image taken from wall of one of the CNTs to show multiple walls, which further confirms the CNTs to be MWCNTs.

Deposition of $\mathrm{Al}_{2} \mathrm{O}_{3}$ on the walls of the CNTs was quite expected in ALD process. TEM images unambiguously establish that ALD precursors successfully deposited $\mathrm{Al}_{2} \mathrm{O}_{3}$ on individual CNTs, penetrating through the open space between CNTs. 

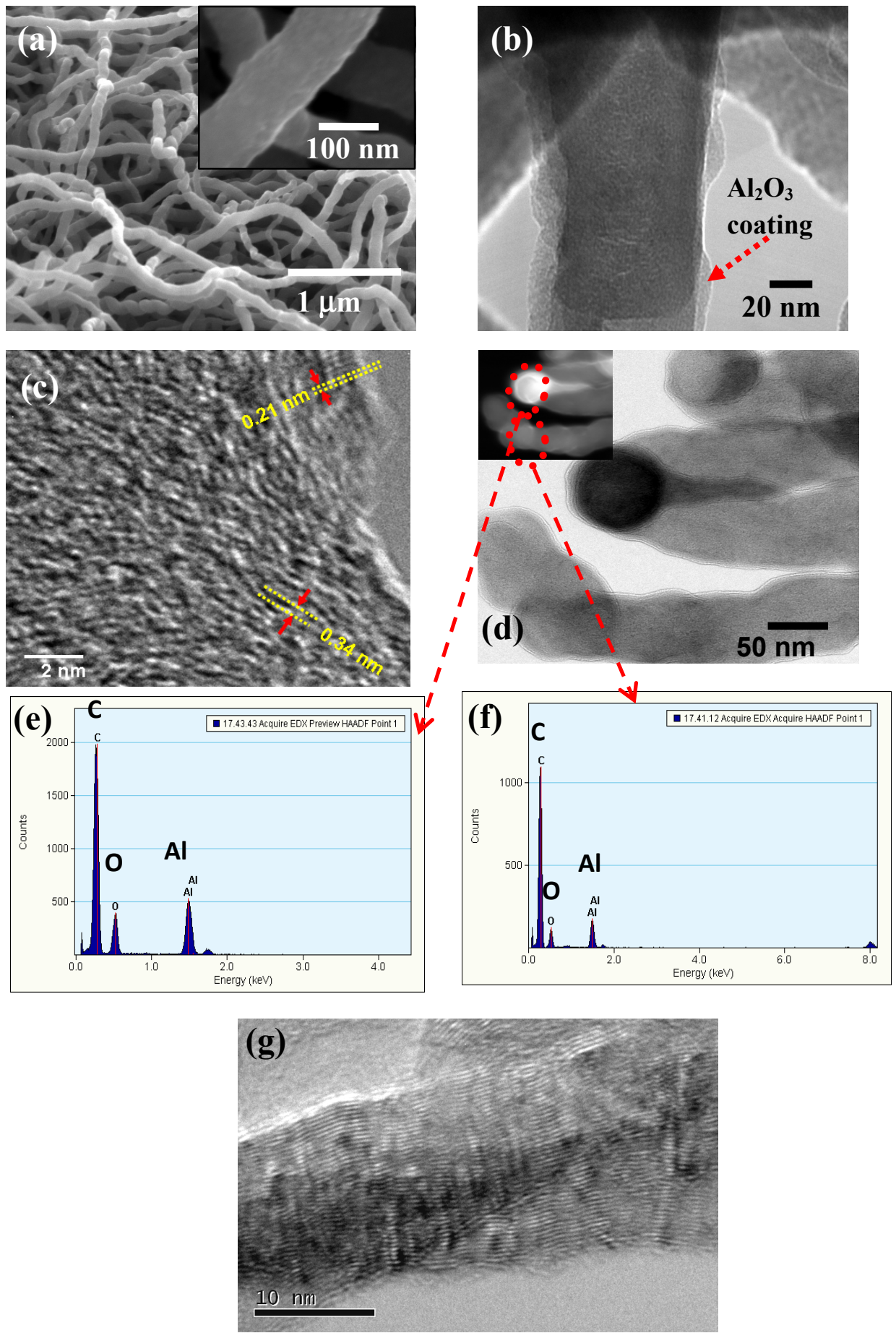

Figure 4.35: Structure of the anode material. (a) SEM images of the ALD alumina coated MWCNT; the inset shows high magnification image of individual MWCNTs. (b) TEM image of individual CNTs, showing the ultra-thin coatings of alumina on CNTs. (c) Lattice fringes showing alumina $(0.21 \mathrm{~nm})$ and CNT $(0.34 \mathrm{~nm})$. (d) TEM images of few 
CNTs. The inset shows the high angle angular dark field scanning transmission electron microscope (HAADF-STEM) image from the same location. (e) and (f) Spot elemental analysis performed at different positions on the wall of a few CNTs. Both analysis show presence of $A l$ and $O$, indicating that alumina has covered the walls of the CNTs forming a thin and discontinuous coating on CNTs. (g) HRTEM image from walls of one of the MWCNTs, showing a presence of multiple walls.

However, formation of $\mathrm{Al}_{2} \mathrm{O}_{3}$ by ALD on CNTs is theoretically not expected on conjugated $\mathrm{C}$ bonds of graphene planes of CNTs. $\mathrm{ALD} \mathrm{Al}_{2} \mathrm{O}_{3}$ formation can be initiated on a hydroxyl terminated surface or on defective sites [152]. A large fraction of structural defects in the pristine CNT, as evident from high (more than unity) $\mathrm{I}_{\mathrm{D}} / \mathrm{I}_{\mathrm{G}}$ ratio in Raman Spectrum (see figure 4.28), ensured $\mathrm{Al}_{2} \mathrm{O}_{3}$ nucleation on CNTs. $\mathrm{Al}_{2} \mathrm{O}_{3}$ is expected to improve the electrochemical response of the anode in Li-ion batteries in two ways: providing extra $\mathrm{Li}^{+}$ion intercalation sites (forming $\mathrm{Li}_{9} \mathrm{Al}_{4}$, leading to a theoretical specific capacity of $2234 \mathrm{mAh} \mathrm{g}^{-1}$ ) [157] and protecting individual CNTs from undesirable reaction with the electrolyte by forming a stable thin oxide layer [147]. Figure 4.36 presents lattice fringe image of a CNT in the lithiated state to show formation of $\mathrm{Li}_{9} \mathrm{Al}_{4}$ on its wall (ICDD PDF card number 00-024-0008 for the crystal structure information of $\mathrm{Li}_{9} \mathrm{Al}_{4}$ ). From this HRTEM image, it is clear that $\mathrm{Al}_{2} \mathrm{O}_{3}$ also takes active part in Li-ion intercalation and hence, contribute directly to the enhancement of specific capacity of the anode. In the following parts, electrochemical performance of the electrode will be discussed, which will further highlight this issue. 


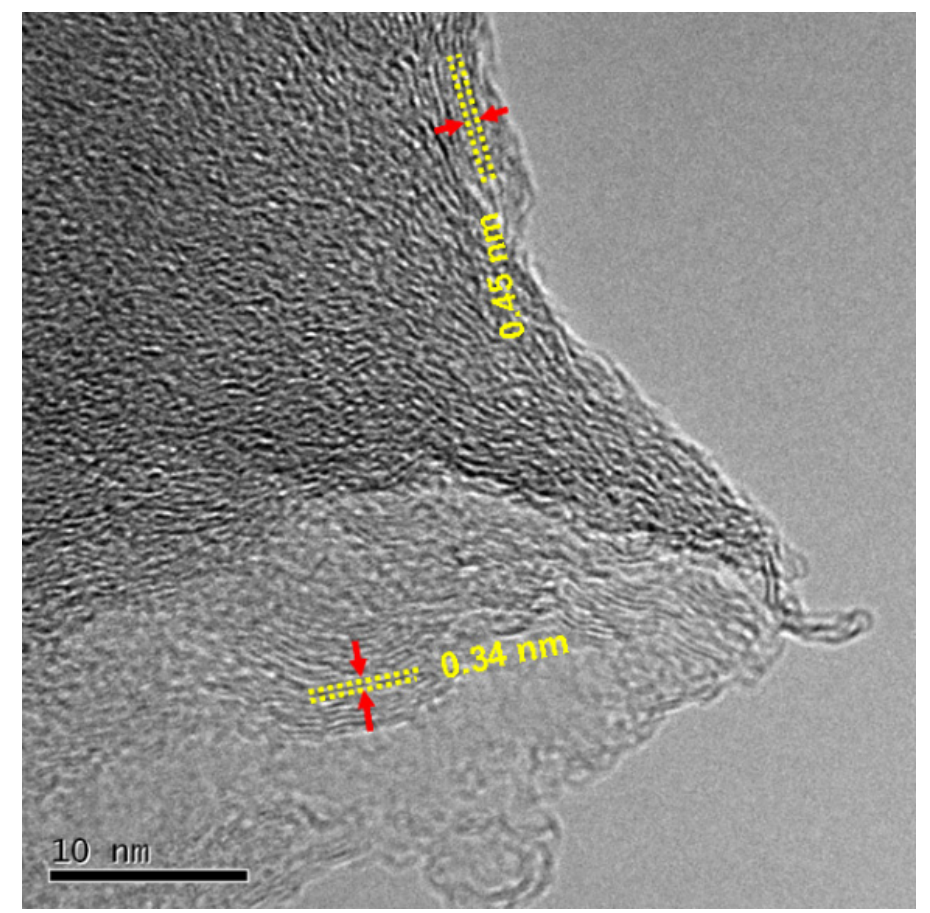

Figure 4.36: Lattice fringe image showing CNTs $(0.34 \mathrm{~nm})$ and (400) plane of $\mathrm{Li}_{9} \mathrm{Al}_{4}$ (0.45 nm) on the outer surface of CNTs (after lithiation).

Electrochemical performance of the half-cells, prepared using the ALD alumina coated CNT structure on $\mathrm{Cu}$ current collectors as working electrode and Li metal foil as reference and counter electrode, are presented in figure 4.37. First two charge-discharge cycles, at a current rate of $38 \mathrm{~mA} \mathrm{~g}^{-1}$, are shown in figure 4.37 (a). First lithiation capacity for the oxide-coated CNT sample was very high (3036 mAh g $\left.{ }^{-1}\right)$. However, first delithiation cycle for the sample showed huge irreversible capacity loss, in the range of $43-45 \%$. This high irreversible capacity loss may be related to solid electrolyte interphase (SEI) formation. However, from the second cycle onwards, irreversible capacity loss was found to be very low. 

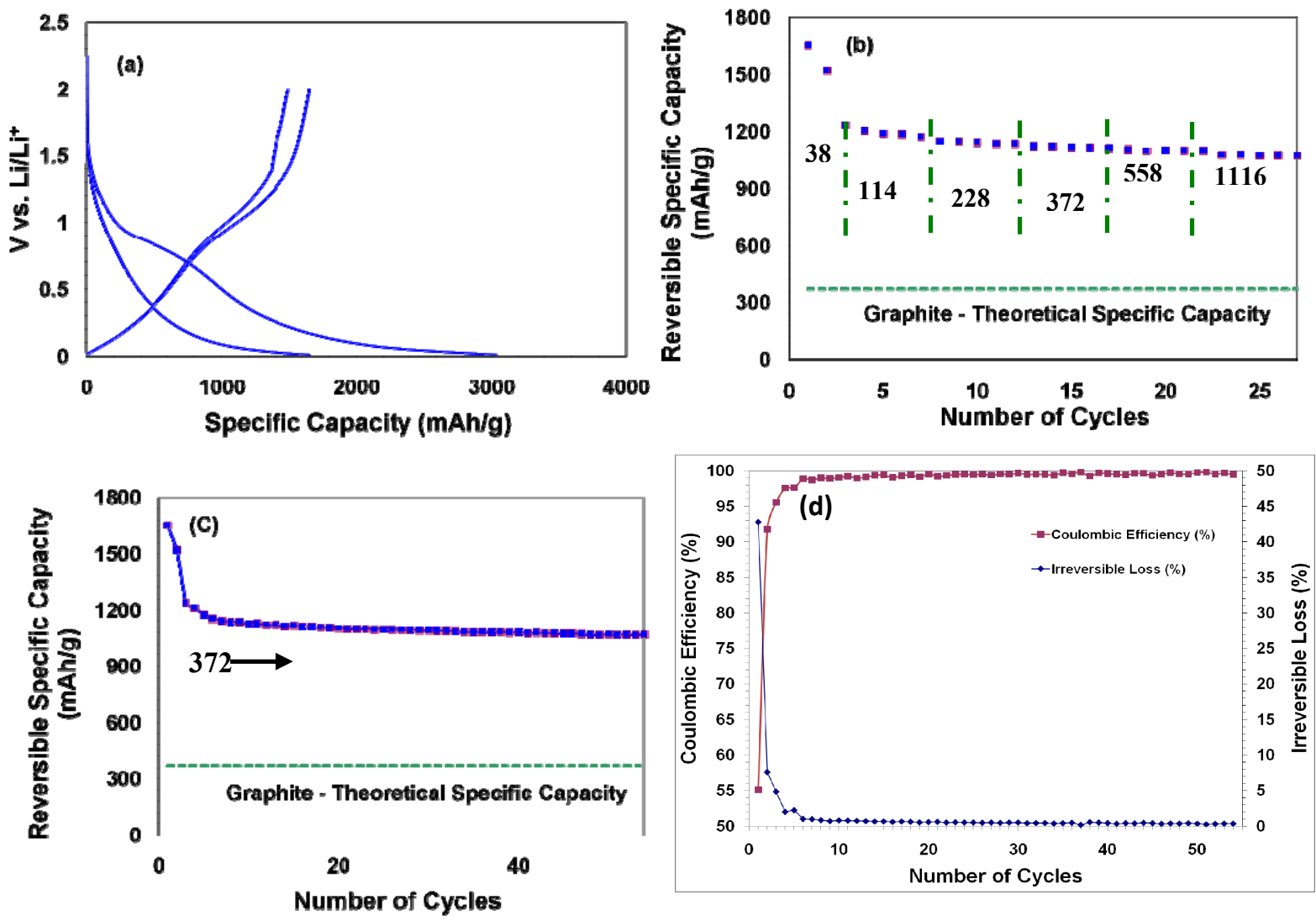

Figure 4.37: Electrochemical performance of the anode material. (a) Charge-discharge behavior of the CNT anode with alumina coating in first two cycles. (b) Rate capability of the alumina-coated CNT anode at five different current rates $(114,228,372,558$ and $1116 \mathrm{~mA} \mathrm{~g}^{-1}$, respectively For comparison, theoretical specific capacity of graphite is included. (c) Specific capacity retention ability of the electrode in higher number of cycles. Numerical values mentioned in figure $b$ and $c$ denotes the current rate $\left(\mathrm{mA}^{-1}\right)$, at which charge-discharge tests are performed. (d) Coulombic efficiency and irreversible capacity loss, as a function of number of cycles.

First lithiation cycle for the alumina-coated CNT anode structures also showed plateau in the range of $1.0-0.5 \mathrm{~V}$. This is a typical signature of SEI formation in Cnanostructure based electrodes [116], which was also observed for the CNT anodes, 
reported in section 4.6. However, it may be noted that the plateau was extended over wider voltage range for the ALD alumina coated CNT anode. This may be related to initial breakdown of the oxide. Absence of the plateau from the second cycle onwards indicates a complete formation of SEI in the first cycle itself. Another important feature of the charge-discharge curves is the availability of a good fraction of full capacity at a voltage higher than $0.5 \mathrm{~V}$. This is again a common feature for all C-nanostructure based anodes and even nano-composite anodes like C-Si nanowire structures [117-120]. Contrary to expectation, this behavior does not affect the full cell characteristics in any significant way [158].

Figure 4.37 (b) presents the rate capability for the ultra-thin alumina-coated CNT anodes. Specific capacities of the electrodes were tested at five different current rates $\left(114,228,372,558\right.$ and $1116 \mathrm{~mA} \mathrm{~g}^{-1}$, respectively), after two initial cycles at $38 \mathrm{~mA} \mathrm{~g}^{-1}$. At all the current rates, oxide coated CNT structure has shown very high specific capacity. It may be noted here that specific capacity of this alumina-coated MWCNT anode was much higher than that offered by bare MWCNT based anode, at all current rates (compare with figure $4.27 \mathrm{~b}$ ). Moreover, the reduction in specific capacity, when tested under higher current rate, is very low for the alumina coated CNTs. Thus, this anode showed a high potential for fast charging, a basic requirement for practical applications. The uncoated CNT anode, on the contrary, showed a typical staircase type nature of the rate capability plot, common to most anode materials (compare with figure $4.27 b)$.

Another important feature for practical application of the anode is its capacity retention in long-cycle operation. Specific capacity of the electrode was tested for more 
than 50 cycles, at a current rate of $372 \mathrm{~mA} \mathrm{~g}^{-1}$ (figure $4.37 \mathrm{c}$ ). It may be observed that the ALD alumina-coated CNT electrode showed stable capacity. The alumina coated CNT anode offered higher capacity $\left(\sim 1100 \mathrm{mAh} \mathrm{g}^{-1}\right)$ than the uncoated CNT anode $(\sim 900$ $\mathrm{mAh} \mathrm{g}^{-1}$ ). Capacity fading for the alumina coated CNT anode showed extremely low (less than $0.1 \%$ per cycle) degradation in specific capacity, which was considered to be comparable to most electrode materials. This electrode also offered very high Coulombic efficiency $(>99 \%)$ and very low irreversible capacity loss $(<1 \%)$, after initial five cycles (figure $4.37 \mathrm{~d}$ ), indicating good battery performance.

ALD alumina coated CNT anodes, directly synthesized on $\mathrm{Cu}$ current collectors, have shown multifarious advantages over conventional MCMB or natural graphite based electrodes (figure 4.38).

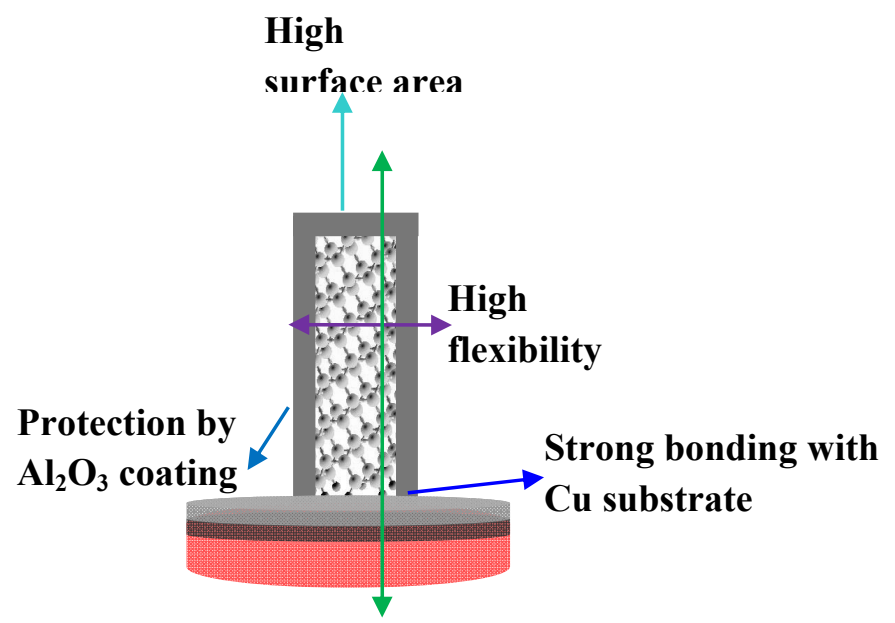

Ultra-low resistance path through CNTs

Figure 4.38: A schematic of the advantageous features of ultrathin alumina coated MWCNT anode for Li-ion batteries.

First, direct growth of interface-controlled MWCNTs on $\mathrm{Cu}$ current collector ensures good CNT-substrate bonding and extremely low resistance path for faster 
electron movement, thus ensuring structural stability and faster charge transport. Second, higher surface area of MWCNTs provide extra $\mathrm{Li}^{+}$ion intercalation sites. A conservative geometrical calculation (considering MWCNTs as cylinders and taking into account of only the outer surface area of outermost wall, their average outer diameter and density) predicts the specific surface area to be $113 \mathrm{~m}^{2} / \mathrm{g}$. It is worthy to mention here that actual surface area of the samples is expected to be much higher - due to contributions from the inner walls of the MWCNTs and defects present in the structure. Third, alumina coating on the CNTs provides further intercalation sites, apart from providing stability to the electrode (by forming an extra layer, which protects CNTs against reactions with the electrolyte). Moreover, alumina coating makes the SEI more stable, thus minimizing the chance of thermal runaway [152]. Further, the binder-free nature of the electrode offers enhanced safety of the battery, as binders are known to decompose at higher temperature and initiate exothermic reactions, leading to explosions [158]. Strong bonding of the CNTs with the $\mathrm{Cu}$ substrate is another favorable issue for this electrode structure, increasing its structural stability during long cycle operation. Finally, specific capacity of the alumina coated CNT anode showed extremely high capacity; even at a very high current rate of $372 \mathrm{~mA} \mathrm{~g}^{-1}$ the anode showed a specific capacity of $\sim 1100 \mathrm{mAh} \mathrm{g}^{-1}$, which was almost 3 times the theoretical specific capacity of graphite. Ultrathin alumina coated MWCNT anode, developed in present study, showed higher specific capacity than any other C-nanostructure based anodes and any type of oxide-containing anodes for $\mathrm{Li}$ ion batteries (figure 4.39). A quick comparison of specific capacities of anodes reveals that both MWCNT-on-Cu and alumina-coated MWCNT-on-Cu anodes show similar or better capacities, compared to most anode materials. 


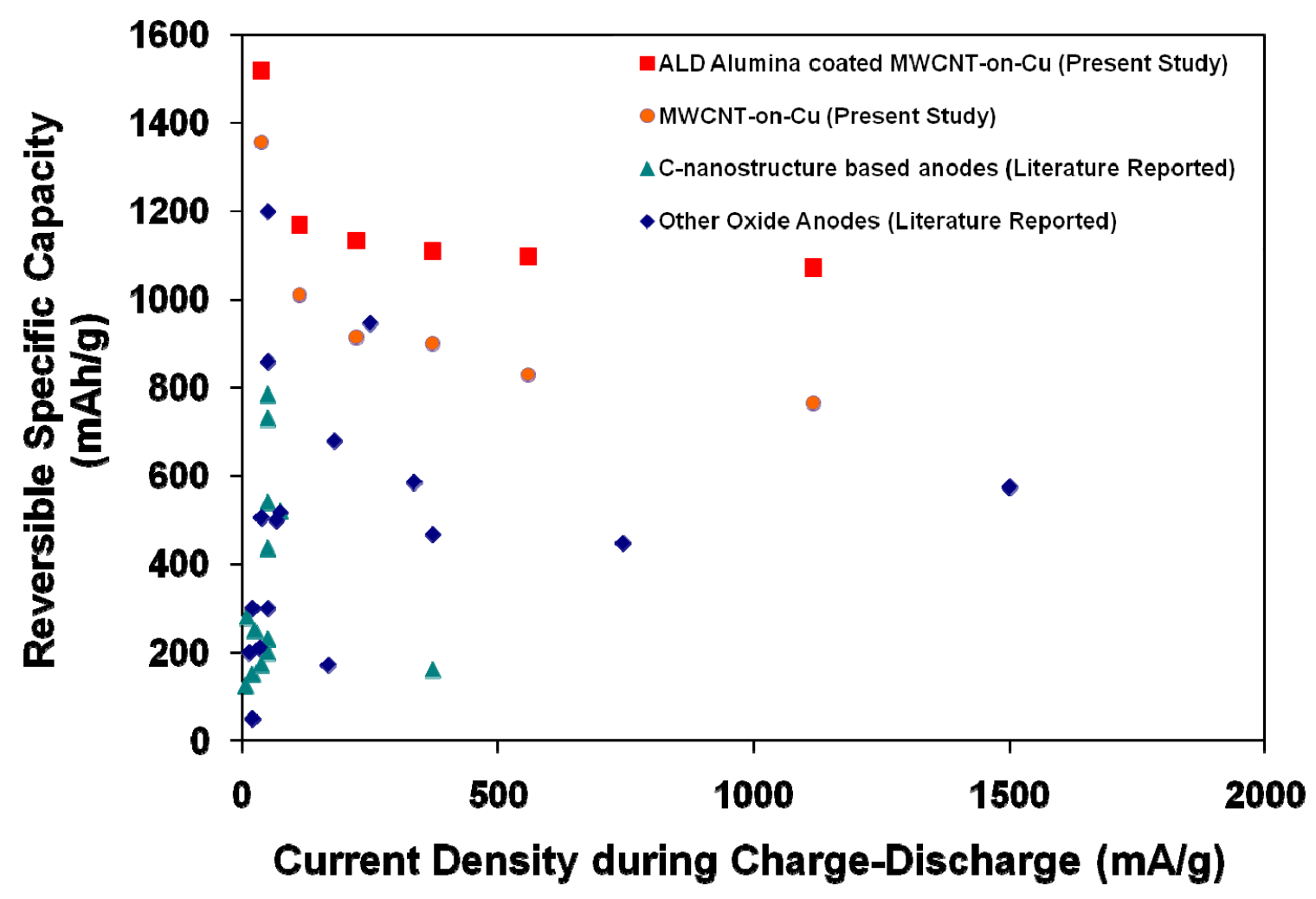

Figure 4.39: A comparison plot showing the specific capacities different anodes (as available in open literature) and that of the anodes in the present study, as a function of current density. [112-114, 116, 117, 119, 120, 125, 135, 138, 139, 140-144, 159-168]

Better performance of these two types of anodes, proposed in the present study, is more evident in higher current density ranges. Thus, these novel nanostructured anodes are promising candidates for possible replacement of anodic material in future Li-ion batteries. Further studies to verify its performance in actual battery system should expedite use of these anodes in practical Li-ion batteries, enabling higher capacity, faster charging and better safety. 


\subsection{Operational Mechanism of Carbon Nanotube Based Anode in Li-ion Battery}

In sections 4.5 and 4.6 , detailed description and analysis have been provided regarding development of two types of novel anode materials for Li-ion battery MWCNT-on-Cu and ultrathin alumina coated MWCNT-on-Cu anodes. From structural and electrochemical characterization, issues like its mechanism, reasons for high capacity and high stability were understood. However, structure of SEI and its stability also dictates long term operational performance and safety of these anodes. Thus, further efforts were made to understand these issues. Differential scanning calorimetry (DSC) can indicate structural stability of the electrode and its performance at higher operational temperature, indicating safety of the electrodes. On the other hand, electrochemical impedance spectroscopy (EIS) can predict about development of any unusual resistance in electron's path, leading to an idea about charge transfer process and probability of generating heat during operation. Further, x-ray photoelectron spectroscopy (XPS) can identify the type of bonds in SEI layer and hence, will be able to predict SEI formation and its stability. In the present study, these three techniques were used to gain further insight into stability and safety issues. The following sub-sections will concentrate on each of these techniques, separately.

\subsubsection{Differential Scanning Calorimetry (DSC) Studies on MWCNT Anode}

DSC studies were performed on the anode samples at different stages of lithiation and de-lithiation. Anode samples were collected after $1^{\text {st }}$ and $2^{\text {nd }}$ cycles, each after lithiation and de-lithiation and characterized for existence for any exothermic peak up to a temperature of $773 \mathrm{~K}\left(500^{\circ} \mathrm{C}\right)$. For comparison purpose, samples are also collected 
during different stages of sample preparation. Results obtained from all these samples are compared in figure 4.40. Data obtained from DSC were compared with the calibration sample to understand its reliability.

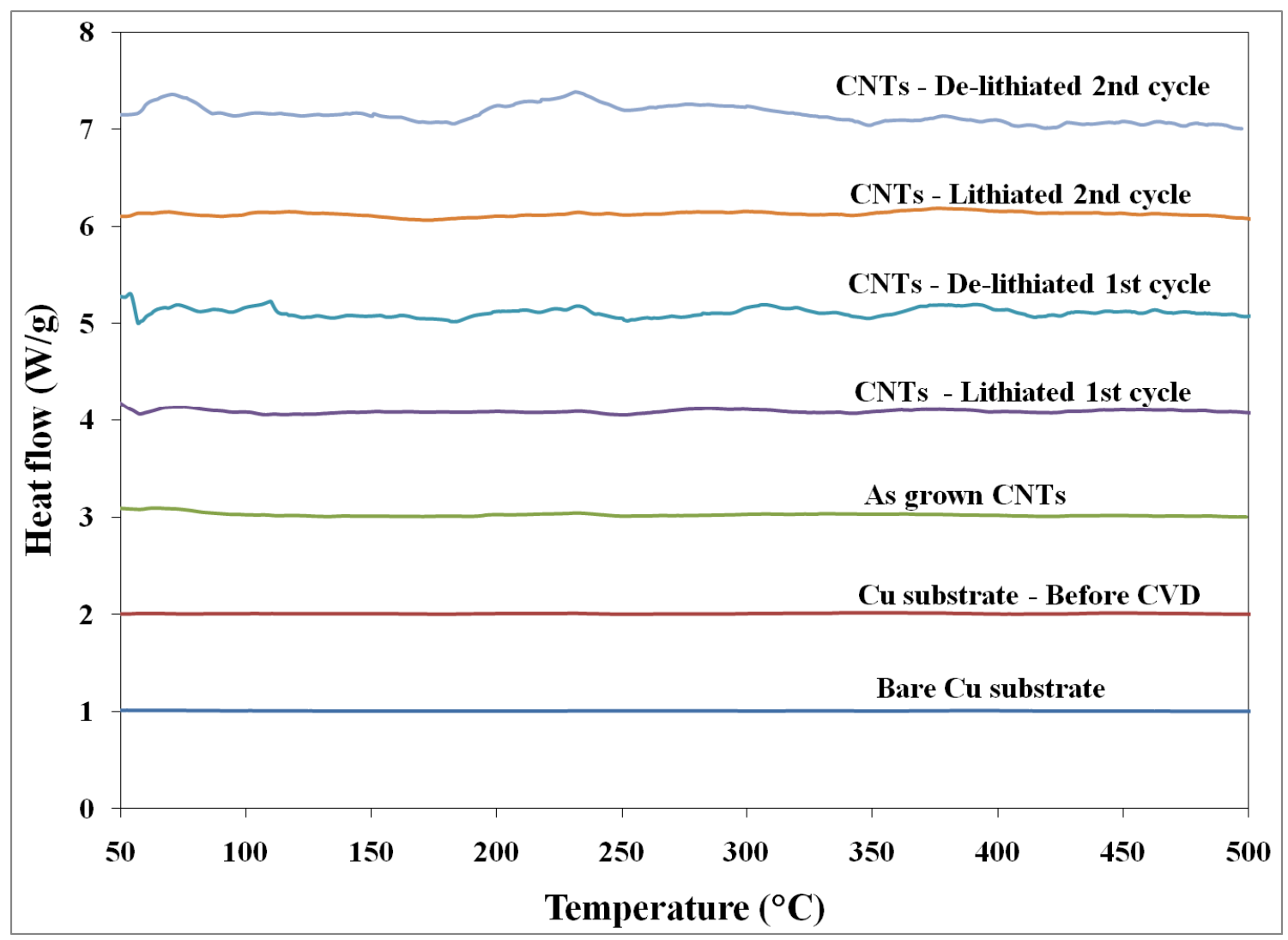

Figure 4.40: Differential scanning Calorimetry plots MWCNT anode, during different stage of preparation and different states of lithiation/de-lithiation.

A quick look at the figure reveals immediately that the MWCNT-on-Cu anodes did not show any appreciable exothermic peak till $500^{\circ} \mathrm{C}$. Some minor peaks were observed in the de-lithiated states; however heat evolved in those peaks were almost negligible. So, it seems that the MWCNT-on-Cu anodes are safe for practical use. The content of the electrode did not undergo any reaction, producing any appreciable amount of heat, which might have led to thermal runaway. However, it is important to investigate 
further into the smaller peaks found on de-lithiated samples. It may be observed that such peaks were absent in the lithiated state and even during all stages of sample preparation. To understand this issue more clearly, it is necessary to check formation of any extra resistance and its possible composition, which can be performed through EIS and XPS analysis. Following two sub-sections will focus on these two kinds of characterization.

\subsubsection{Electrochemical Impedance Spectroscopy (EIS) Studies on MWCNT Anode}

EIS studies are becoming increasingly popular for electrochemical systems, though interpretation of the EIS plots and explaining basic electrical circuit elements is still not easily understandable to all $[169,170]$. In the present study, EIS was performed for half cells with different charging/discharging history and results are explained in order to understand internal mechanism. It may be recalled here from chapter 3 that during the present study, all EIS tests were done in galvanostatic mode, using a $10 \mu \mathrm{A}$ (rms value) ac signal, within the frequency domain of $100 \mathrm{kHz}$ to $1 \mathrm{mHz}$. Samples with five (5) different charging history were tested $-1^{\text {st }}$ cycle after lithiation, $1^{\text {st }}$ cycle after delihiation, $2^{\text {nd }}$ cycle after lithiation, $2^{\text {nd }}$ cycle after de-lithiation and $55^{\text {th }}$ cycle after delithiation. Before onset of measurement, each half cell was equilibrated for $100 \mathrm{~s}$ at open circuit voltage (OCV). Figure 4.41 summarizes the EIS output, in terms of Nyquist plots and Bode plots, which are the conventional methods of explaining EIS data. The plots were compared with available theory for EIS to understand their meaning. Equivalent circuit models are proposed after analysis of the data. 

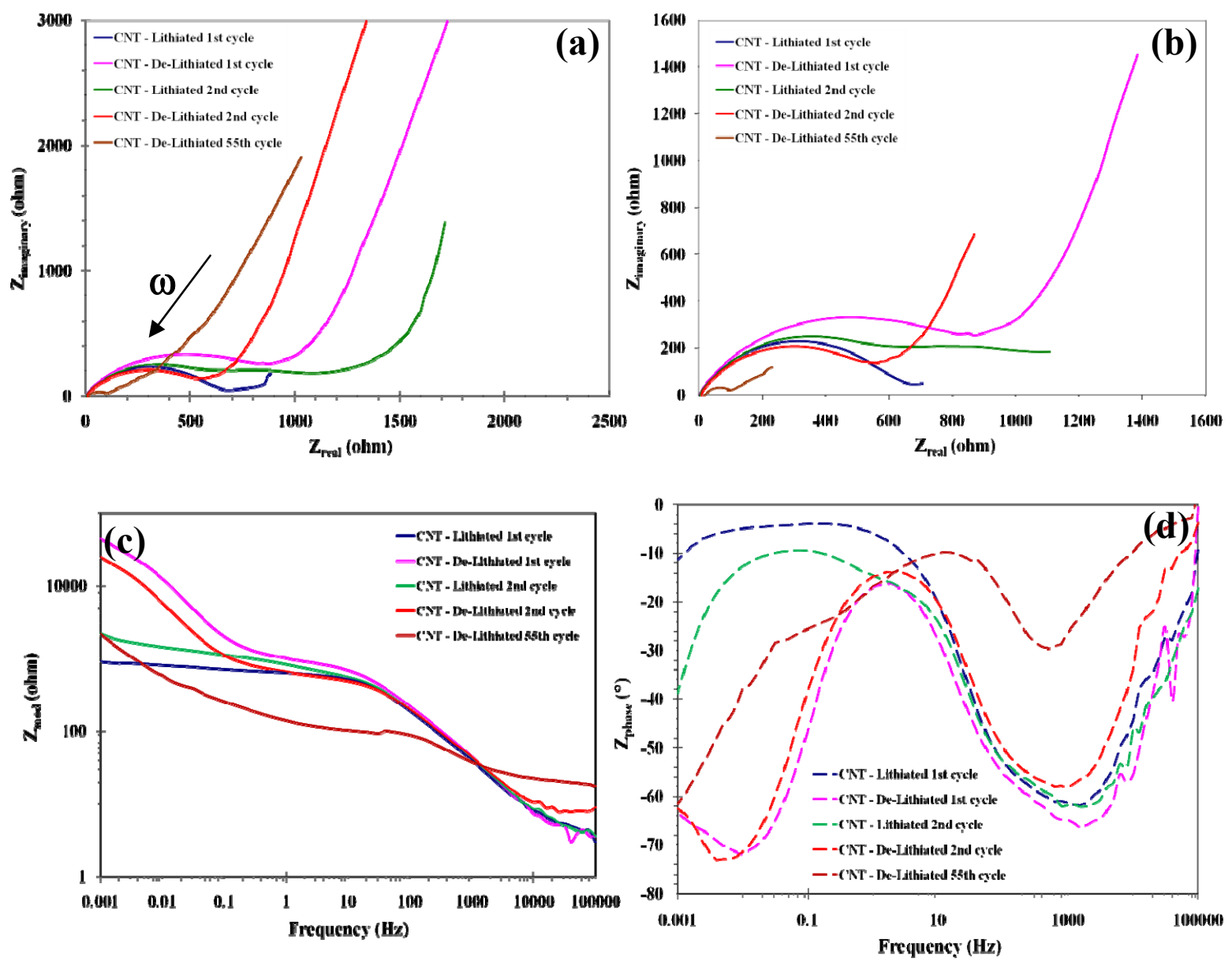

Figure 4.41: Electrochemical impedance spectroscopy results for MWCNT-on-Cu anodes, at different conditions of charge/discharge. (a) Nyquist plot for the whole frequency domain. (b) Nyquist plot for the frequency range $10 \mathrm{kHz}-100 \mathrm{mHz}$. (c) and (d) are Bode plots showing absolute impedance and phase-shift.

A quick look at the Nyquist plots, figure 4.41 (a), show that EIS responses from all the samples have a semi-circular part and a straight line part, which makes approximately $45^{\circ}$ angle with the horizontal axis. It becomes obvious that the latter part, straight line with roughly $45^{\circ}$ angle with $\mathrm{x}$-axis, is due to Warburg impedance, which is 
known as the impedance caused by diffusional movement of species. Warburg impedance is affected by the frequency of applied signal - at high frequencies, this impedance is small as diffusing species don't have to move very far. On the other hand, Warburg impedance is substantial at lower frequencies, as reactants have to diffuse further. In the present case also, same trend is observed, which is more visible in figure 4.41 (b), in which a certain portion of the low frequency results have been purposefully omitted to show this effect. Even in such a case, it is observed that Warburg impedance is negligible for lithiated samples, but appreciable for all de-lithiated samples. This common trend indicates that products of de-lithiation cause more impedance in the cell.

Comparison of the Nyquist and Bode plots of this anode material with case histories, reported in open literature [171-176], gives an idea of the equivalent circuit for the present system. Figure 4.42 shows the basic equivalent circuit.

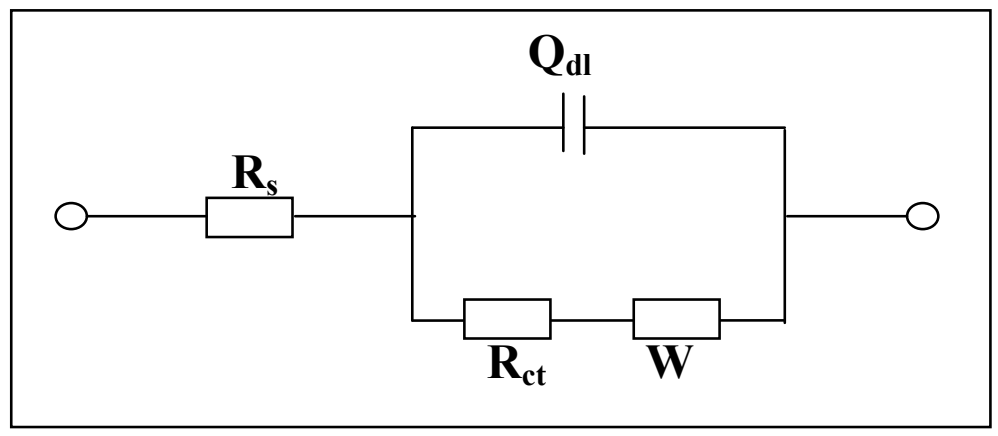

Figure 4.42: Equivalent circuit with mixed charge transfer and diffusion control, for MWCNT-on-Cu anodes.

In figure $4.42, R_{s}$ is ohmic resistance of the cell, including resistance of current collectors, active materials, electrolyte and separator; $\mathrm{Q}_{\mathrm{d} l}$ is the capacitance for the constant phase element (double-layer capacitance - it is called constant phase element, as 
it behaves little differently than ideal capacitors in electrochemical cells); $\mathrm{R}_{\mathrm{ct}}$ is the charge transfer resistance and $\mathrm{W}$ is the Warburg impedance. An equivalent circuit, which does not have this "W" term, shows a typical semi-circle behavior in Nyquist plot. Considering only the semi-circular part of the Nyquist, values of solution resistance and charge transfer resistance can be appreciated. Solution resistance is given by the intercept value between the plot and the real impedance axis (at highest frequency), while diameter of the semi-circle is the charge transfer resistance. It may be observed from figure 4.41 that the solution resistance $\left(R_{s}\right)$ is very low, between $3-5 \Omega$, for all the samples and it remains nearly constant even after 55 cycles. However, charge transfer resistance $\left(\mathrm{R}_{\mathrm{ct}}\right)$ varies extensively between different samples. During the initial cycles, charge transfer resistance is very high, while it shrinks to low value after 55 cycles. This trend indicates better performance of the anode with cycling, which is evident from the excellent capacity stability during cycling. Another important issue to be observed from figure 4.41 (a) is that the semi-circular parts of the 'lithiated' samples could be approximated as super-position of two semi-circles, while for the 'de-lithiated' samples, it is clearly one semi-circle. While the first semi-circle (at high frequency end) is associated commonly with SEI, the second semi-circle has often been related to presence of an extra doublelayer capacitance [174] or an extra interface [172]. Sometimes, such second semi-circles are related to any extra reaction front created by a failing coating [173]. In the present case, decreasing charge transfer resistance with increasing number of cycle does not indicate any failure of SEI. However, with available data, it is difficult to conclude about the source of such second semi-circles in 'lithiated' samples. 


\subsubsection{X-ray Photoelectron Spectroscopy (XPS) Studies on MWCNT Anode}

XPS aids in predicting possible composition of the SEI layer, formed on MWCNTs, during charging and discharging processes. A systematic study of several samples, after different cycles, can also highlight the stability of the SEI. Since, SEI stability is known to be associated with battery performance, analysis of XPS data is expected to give valuable information about the stability of the electrode. Figure 4.43 presents different XPS spectra of a number of samples. Peaks are identified using several references [171, 177-179].

For understanding the composition of SEI, each peak in the high resolution scans of Li 1s, C 1s, O 1s, F 1s and P 2p energy spectra needs to be identified. In the present case, $\mathrm{Li} 1 \mathrm{~s}$ spectra showed presence of $\mathrm{Li}_{2} \mathrm{CO}_{3}$ in all the samples. Peak from this compound was also found in $\mathrm{C} 1 \mathrm{~s}$ and $\mathrm{O}$ 1s spectra. Its amount also does not change much, with increasing number of cycles and conditions of charge or discharge. Thus, it is concluded that $\mathrm{Li}_{2} \mathrm{CO}_{3}$ was a permanent component of SEI, irrespective of number of cycles or state of charge/discharge. $\mathrm{Li}_{2} \mathrm{CO}_{3}$ is a known component in graphite-based anodes. Several theories exist explaining formation of this compound - all of them indicate that it is a product of decomposition of electrolyte (EC or DEC) and reaction with $\mathrm{Li}^{+}$ions [177]. Hydrocarbons (C-C or $\mathrm{C}-\mathrm{H}$ type bonds) were also present in all the samples, which was a very common and expected observation. C 1s spectra showed one prominent peak in the range of $287-288 \mathrm{eV}$, which could either be related to lithium alkyl carbonates $\left(\mathrm{R}-\mathrm{CH}_{2} \mathrm{OCO} 2 \mathrm{Li}\right)$ and lithium ethers $\left(\mathrm{R}-\mathrm{CH}_{2} \mathrm{OLi}\right)$ or simply with $\mathrm{C}-\mathrm{O}$ type bonds. However, corresponding energy peaks of these bonds are not observed in Li 1s and $\mathrm{O}$ 1s spectra. Thus, at this moment, it is not possible to specifically identify this peak. 


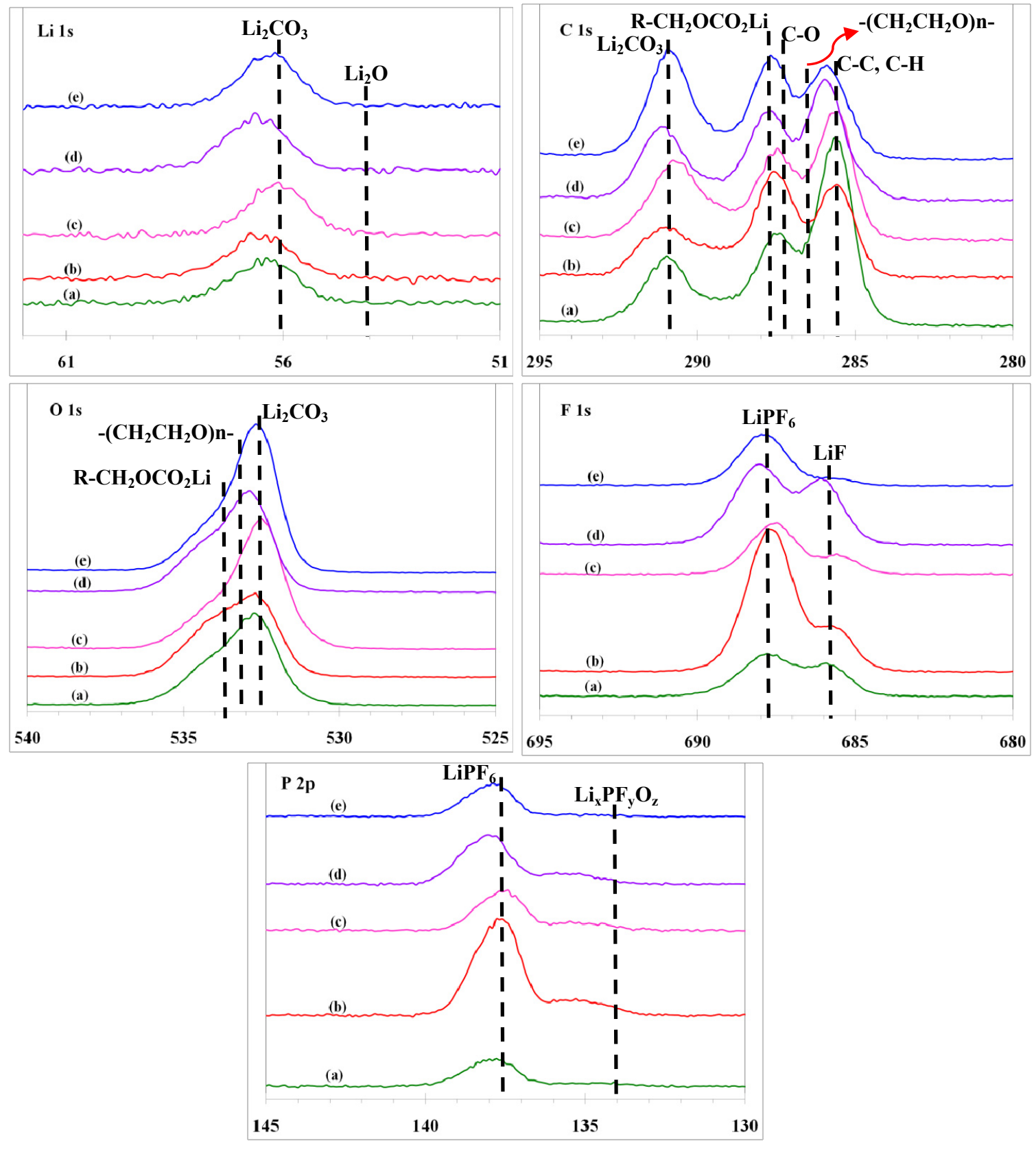

Figure 4.43: $L i 1 s, C 1 s, O 1 s, F 1 s$ and $P 2 p$ XPS spectra of the anode after (a) $1^{s t}$ lithiation, (b) $1^{\text {st }}$ de-lithiation, (c) $2^{\text {nd }}$ lithiation, (d) $2^{\text {nd }}$ de-lithiation and (e) $55^{\text {th }}$ delithiation cycles. 
Both F 1s and P 2p spectra showed peaks for P-F bonds ( $\left.\mathrm{LiPF}_{6}\right)$. From both the spectra, it was clear that it started forming from $1^{\text {st }}$ lithiation cycle itself and showed highest concentration after $1^{\text {st }}$ discharge cycle. After this cycle, its concentration went down and remained almost constant even up to $55^{\text {th }}$ cycle. One important issue to be observed from F 1s spectra was the peak for LiF. Though it was found in both charged and discharged conditions till $2^{\text {nd }}$ cycle, its absence in $55^{\text {th }}$ cycle indicates that LiF was not a stable part of SEI and during cycling, it probably decomposed to form other products. Formation of $\mathrm{LiF}$, either by reaction with trace water or with $\mathrm{Li}_{2} \mathrm{CO} 3$, was not directly driven by electrochemical lithium insertion [177] and probably during long cycling, it reacted back to form $\mathrm{Li}_{2} \mathrm{CO}_{3}$ and $\mathrm{LiPF}_{6}$.

In figure 4.43, possible positions of some other bonds are identified, such as polyethylene oxide (PEO) type oligomers with a structure of $-\left(\mathrm{CH}_{2} \mathrm{CH}_{2} \mathrm{O}\right)_{\mathrm{n}^{-}}$, lithium containing phosphates $\left(\mathrm{Li}_{x} \mathrm{PF}_{y} \mathrm{O}_{z}\right)$, lithium oxide o the $\left(\mathrm{Li}_{2} \mathrm{O}\right)$. Though these bonds are often found in SEI formed on graphite anodes in Li-ion batteries, none of these were observed in the present case. This is in direct contrast to the observations made for graphite electrodes. However, it may be stressed at this moment that SEI on MWCNT anodes has not been analyzed before. So, it may be concluded that composition of SEI on MWCNT anode is little bit different from that on graphite electrode, though it is found to be very stable even up to 55 cycles.

Detailed analysis through DSC, EIS and XPS aided in understanding the composition and stability of SEI, which were helpful in explaining excellent properties of MWCNT-on-Cu anode for application Li-ion batteries. 


\section{References}

1. W.A. de Heer, A. Chatelain, D. Ugarte. A Carbon Nanotube Field-Emission Electron Source. 1995, Science, Vol. 270, pp. 1179-1180.

2. Q.H. Wang, A.A. Setlur, J.M. Lauerhaas, J.Y. Dai, E.W. Seelig, R.P.H. Chang. $A$ nanotube-based field-emission flat panel display. 1998, Appl. Phys. Lett., Vol. 72, pp. 2912-2913.

3. P.G. Collins, A. Zettl. A simple and robust electron beam source from carbon nanotubes. 1996, Appl. Phys. Lett., Vol. 69, pp. 1969-1971.

4. H. Dai. Carbon Nanotubes: Synthesis, Integration, and Properties. 2002, Acc. Chem. Res., Vol. 35 , pp. 1035-1044.

5. A.A. Kuznetzov, S.B. Lee, M. Zhang, R.H. Baughman, A.A. Zakhidov. Electron field emission from transparent multiwalled carbon nanotube sheets for inverted field emission displays. 2010, Carbon, Vol. 48, pp. 41-46.

6. R. Seelaboyina, S. Boddepalli, K. Noh, M. Jeon, W. Choi. Enhanced field emission from aligned multistage carbon nanotube emitter arrays. 2008, Nanotechnology, Vol. 19, p. 065605.

7. R. Seelaboyina, J. Huang, W.B. Choi. Enhanced field emission of thin multiwall carbon nanotubes by electron multiplication from microchannel plate. 2006, Appl. Phys. Lett., Vol. 88, p. 194104.

8. R. Seelaboyina, I. Lahiri, W. Choi. Carbon-nanotube-embedded novel threedimensional alumina microchannel cold cathodes for high electron emission. 2010, Nanotechnology, Vol. 21, p. 145206.

9. B. Ribaya, D. Niemann, N. Gunther, M. Rahman, C.V. Nguyen. An experimental study and modeling of the field emission propperties of an isolated individual multiwalled carbon nanotube. 2006, IEEE Conf. Proc., pp. 505-506.

10. K.A. Dean, B.R. Chalamala. The environmental stability of field emission from single-walled carbon nanotubes. 19, 1999, Appl. Phys. Lett., Vol. 75, pp. 30173019.

11. S. Kato, T. Noguchi. Carbon nanotubes and related field emitters: Fundamentals and applications. [ed.] Y. Saito. Wiley-VCH, 2010. pp. 373-376.

12. T. Noguchi. Study on enhancement of field emission characteristics for multiwalled carbon nanotubes. Sokendai University (Japan). 2004. Doctoral thesis.

13. S. Talapatra, S. Kar, S.K. Pal, R Vajtai, L. Ci, P. Victor, M.M. Shaijumon, S. Kaur, O. Nalamasu, P. M. Ajayan. Direct growth of aligned carbon nanotubes on bulk metals. 2006, Nature nanotechnol., Vol. 1, pp. 112-116. 
14. R. Saito, G. Dresselhaus, M.S. Dresselhaus. Physical properties of carbon nanotubes. Imperial College Press, London (UK), 1998.

15. K. Barbalace. Periodic table of elements - sorted by electrical conductivity. EnvironmentalChemistry.com.

[Online] www.environmetalchemistry.com/yogi/periodic/electrical.html.

16. T.-Y. Tsai, N.-H. Tai, I.-N. Lin. Characteristics of carbon nanotube electron field emission devices prepared by LTCC process . 2004, Diamond Relat. Mater. , Vol. 13, pp. 982-986.

17. M. Dubosc, S. Casimirius, M.-P. Besland, C. Cardinaud, A. Granier, J.-L. Duvail, A. Gohier, T. Minéa, V. Arnal, J. Torres. Impact of the Cu-based substrates and catalyst deposition techniques on carbon nanotube growth at low temperature by PECVD . 2007, Microelectron. Eng., Vol. 84, pp. 2501-2505.

18. N. Chiodarelli, Y. Li, D.J. Cott, S. Mertens, N. Peys, Heyns M, S. de Gendt, G. Groeseneken, P.M. Vereecken. Integration and electrical characterization of carbon nanotube via interconnects . 2011, Microelectron. Eng., Vol. 88, pp. 837843.

19. D. Kim, S.H. Lim, A.J. Guilley, C.S. Cojocaru, J.E. Bourée, L. Vila, J.H. Ryu, K.C. Park, J. Jang. Growth of vertically aligned arrays of carbon nanotubes for high field emission. 2008, Thin Solid Films, Vol. 516, pp. 706-709.

20. X. Yin, Q. Wang, C. Lou, X. Zhang, W. Lei. Growth of multi-walled CNTs emitters on an oxygen-free copper substrate by chemical-vapor deposition. 2008, Appl. Surf. Sci., Vol. 254, pp. 6633-6636.

21. T. Ikuno, S.I. Honda, H. Furuta, K. Aoki, T. Hirao, S. Ohkura, M. Katayama. Correlation between field electron emission and structural properties in randomly and vertically oriented carbon nanotube films. 2005, Jap. J. Appl. Phys., Vol. 44, pp. 1655-1660.

22. N. Chiodarelli, S. Masahito, Y. Kashiwagi, Y. Li, K. Arstila, O. Richard, D.J. Cott, M. Heyns, S. de Gendt, G. Groeseneken, P.M. Vereecken. Measuring the electrical resistivity and contact resistance of vertical carbon nanotube bundles for application as interconnects . 2011, Nanotechnology, Vol. 22 , p. 085302.

23. Y. Chai, Z. Xiao, C.H. Chan. Horizontally aligned carbon nanotube bundles for interconnect application: diameter-dependent contact resistance and mean free path. 2010, Nanotechnology, Vol. 21, p. 235705.

24. A. Smolyanitsky, V.K. Tewary. Simulation of lattice strain due to a CNT-metal interface. 2011, Nanotechnology, Vol. 22, p. 085703. 
25. N.S. Xu, J. Chen, S.Z.Deng. Physical origin of nonlinearity in the FowlerNordheim plot of field-induced emission from amorphous diamond films: Thermionic emission to field emission. 2000, Appl. Phys. Lett. , Vol. 76, pp. 24632465 .

26. J. Zhang, X. Wang, W. Yang, W. Yu, T. Feng, Q. Li, X. Liu, C. Yang. Interaction between Carbon Nanotubes and Substrate and its Implication in Field Emission Mechanism. 2006, Carbon, Vol. 44, pp. 418-422.

27. E. Minoux, O. Groening, K.B.K. Teo, S.H. Dalal, L. Gangloff, J.P. Schnell, L. Hudanski, I. Y. Y. Bu, P. Vincent, P. Legagneux, G. A. J. Amaratunga, W. I. Milne. Achieving High-Current Carbon Nanotube Emitters. 2005, Nano Lett., Vol. 5, pp. 2135-2138.

28. R.H. Fowler, L.W. Nordheim. Electron emission in intense electric fields. 1928, Proc. R. Soc. London, Vol. A119, pp. 173-181.

29. R.G. Forbes. Field-induced electron emission from electrically nanostructured heterogeneous (ENH) materials. 2001, Ultramicroscopy, Vol. 89, pp. 7-15.

30. W. Wu, S. Krishnan, T. Yamada, X. Sun, P. Wilhite, R. Wu, K. Li, C.Y. Yang. Contact resistance in carbon nanostructure via interconnects. 2009, Appl. Phys. Lett., Vol. 94, p. 163113.

31. P.H. Cutler, N.M. Miskovsky, P.B. Lerner, M.S. Chung. The use of internal field emission to inject electronic charge carriers into the conduction band of diamond films: a review. 1999, Appl. Surf. Sci., Vol. 146, pp. 126-133.

32. H.B. Michaelson. The work function of the elements and its periodicity. 1977, J. Appl. Phys., Vol. 48, pp. 4729-4733.

33. Metals handbook, 8th Ed., Vol. 8: Metallography, structures and phase diagrams. American Society for Metals, Ohio (USA), 1973.

34. S.O. Kasap. Principles of electronic materials and devices, 2nd ed. McGraw-Hill, New York (USA), 2002.

35. H. Okamoto. Cu-Ti (Copper-Titanium). 2002, J. Phase Equilibria, Vol. 23, pp. 549550 .

36. P.L. Hansen, J.B. Wagner, S. Helveg, J.R. Rostrup-Nielson, B.S. Clausen, H. Topsoe. Atom-resolved imaging of dynamic shape changes in supported copper nanocrystals. 2002, Science, Vol. 295 , pp. 2053-2055.

37. A. Maiti, A.Ricca. Metal-nanotube interactions - Binding energies and wetting properties. 2004, Chem. Phys. Lett., Vol. 395, pp. 7-11. 
38. Y. Nosho, S. Kishimoto, T. Mizutani. Relation between conduction property and work function of contact metal in carbon nanotube field-effect transistors. 2006, Nanotechnology, Vol. 17, pp. 3412-3415.

39. E. Durgun, S. Dag, V.M.K. Bagci, O. Gülseren, T.,Yildirim, S. Ciraci. Systematic study of adsorption of single atoms on a carbon nanotube. 2003, Phys. Rev. B , Vol. 67, pp. 2014011-2014014.

40. C. Zhou, J. Kong and H. Dai. Intrinsic electrical properties of individual singlewalled carbon nanotubes with small band gaps. 2000, Phys. Rev. Lett., Vol. 84, pp. 5604-5607.

41. P.M. Ryan, A.S. Verhulst, D. Cott, A. Romo-Negreira, T. Hantschel, J.J. Boland. Optimization of multi-walled carbon nanotube-metal contacts by electrical stressing. 2010 , Nanptechnology, Vol. 21 , p. 045705.

42. Q. Ngo, D. Petranovic, S. Krishnan, A.M. Cassell, Q. Ye, J. Li, M. Meyyappan, C.Y. Yang. Electron transport through metal-multiwall carbon nanotube interfaces. 2004, IEEE Trans. Nanotechnology, Vol. 3, pp. 311-.

43. J.M. Bonard, C. Klinke, K.A. Dean, B.F. Coll. Degradation and failure of carbon nanotube field emitters. 2003, Phys. Rev. B, Vol. 67, p. 115406.

44. Z. Chen, Q. Zhang, P. Lan, B. Zhu, T. Yu, G. Cao, D.D. Engelsen. Ultrahigh-current field emission from sandwich-grown well-aligned uniform multi-walled carbon nanotube arrays with high adherence strength. 2007, Nanotechnology, Vol. 18, p. 265702 (6).

45. Y.D. Lee, K.-S. Lee, Y.-H. Lee, B.-K. Ju. Field emission properties of carbon nanotube film using a spray method. 2007, Appl. Surf. Sci, Vol. 254, pp. 513-516.

46. N.S. Xu, R.V. Latham and Y. Tzeng, Field-dependence of the area-density of 'COLD' electron emission sites on broad-area CVD diamond films. 1993, Electron Lett., Vol. 29, pp. 1596-1598.

47. B.S. Satyanarayana, A. Hart, W.I. Milne, J. Robertson. Field emission from tetrahedral amorphous carbon. 1997, Appl. Phys. Lett., Vol. 71, pp. 1430-1432.

48. A.N. Obraztsov, A.P. Volkov, A.I. Boronin, S.V. Kosheev. Defect induced lowering of work function in graphite-like materials. 2002, Diamond Related Mater., Vol. 11, pp. 813-818.

49. K.B.K. Teo, M. Chhowalla, G.A.J. Amaratunga, W.I. Milne, G. Pirio, P. Legagneux, F. Wyczisk, D. Pribat, D.G. Hasko. Field emission from dense, sparse, and patterned arrays of cacrbon nanofibers. 2002, Appl. Phys. Lett., Vol. 80, pp. 20112013. 
50. J. Li, W. Zheng, C. Gu, Z. Jin, Y. Zhao, X. Mei, Z. Mu, C. Dong, C. Sun. Field emission enhancement of amorphous carbon films by nitrogen-implantation. 2004, Carbon, Vol. 42, pp. 2309-2314.

51. L. Wang, X. Wang, L. Wang, L. Zhang. Field emission characteristics of nanostructured carbon films deposited on differently pretreated Mo films. 2008, Appl. Surf. Sci., Vol. 255, pp. 3257-3262.

52. X. Yin, Q. Wang, C. Lou, X. Zhang, W. Lei. Growth of multi-walled CNTs emitters on an oxygen-free copper substrate by chemical-vapor deposition. 2008, Appl. Surf. Sci., Vol. 254, pp. 6633-6636.

53. C.J. Lee, J. Park, S.Y. Kang, J.H. Lee. Growth and field electron emission of vertically aligned multiwalled carbon nanotubes. 2000, Chem. Phys. lett., Vol. 326, pp. $175-180$.

54. T. Ikuno, T. Yamamoto, M. Kamizono, S. Takahashi, H. Furuta, S.I. Honda, S. Ohkura, M. Katayama, T. Hirao, K. Oura. Large field emission from carbon nanotubes grown on patterned catalyst thin film by thermal chemical vapor deposition. 2002, Physica B, Vol. 323, pp. 171-173.

55. S. Chhoker, S.K. Arora, P. Srivastava, V.D. Vankar. Electron field emission from graphitic nanoflakes grown over vertically aligned carbon nanotubes. 2008, J. Nanosci. Nanotechnol., Vol. 8, pp. 4309-4313.

56. T. Chen, L.L. Wang, Y.W. Chen, W.X. Que, Z. Sun. Field emission properties of carbon nanotubes film grown on NiCr alloy films. 2007, Appl. Surf. Sci., Vol. 253, pp. 7046-7049.

57. H. Furuta, T. Ikuno, N. Shikina, S. Honda, T. Hirao, K. Oura. Ni-based catalytic growth of vertically aligned multi-walled carbon nanotubes by dual-RF plasma CVD method and their field emission properties. 2002, Physica B, Vol. 323, pp. 299-302.

58. M. Kumar, T. Okazaki, M. Hiramatsu, Y. Ando. The use of camphor-grown carbon nanotube array as an efficient field emitter. 2007, Carbon, Vol. 45, pp. 1899-1904.

59. A.L. Musatov, N.A. Kiselev, D.N. Zakharov, E.F. Kukovitskii, A.I.Zhbanov, K.R. Israel' yants, E.G. Chirkova. Field electron emission from nanotube carbon layers grown by CVD process. 2001, Appl. Surf. Sci., Vol. 183, pp. 111-119.

60. W.I. Milne, K.B.K. Teo, M. Chhowalla, G.A.J. Amaratunga, D. Pribat, P. Legagneux, G. Pirio, Vu Thien Binh, V. Semet. Electron emission from arrays of carbon nanotubes/fibres. 2002, Curr. Appl. Sci., Vol. 2, pp. 509-513. 
61. M.C. Rossi, S. Salvatori, P. Ascarelli, E. Cappelli, S. Orlando. Effect of nanostructure and back contact material on the field emission properties of carbon films. 2002, Diam. Rel. Mater., Vol. 11, pp. 819-823.

62. C.J. Huang, Y.K. Chih, J. Hwang, A.P. Lee, C.S. Kou. Field emission from amorphous-carbon nanotips on copper. 2003, J. Appl. Phys., Vol. 94, pp. 67966800 .

63. Z. Chen, D. den Engelsen, P.K. Bachmann, V. van Elsbergen, I. Koehler, J. Merikhi, D.U. Wiechert. High emission current density microwave-plasma-grown carbon nanotube arrays by post depositional radio-frequency oxygen plasma treatment. 2005, Appl. Phys. Lett., Vol. 87, pp. 243104(1-3).

64. D.Y. Kim, J.B. Yoo, I.T. Han, H.J. Kim, H.J. Kim, J.E. Jung, Y.W. Jin, J.M. Kim, K.H. Chin. The density control of carbon nanotubes using spin-coated nanoparticle and its application to the electron emitter with triode structure. 2005, Diam. Rel. Mater., Vol. 14, pp. 2084-2088.

65. N.A. Kiselev, A.L. Musatov, E.F. Kukovitskii, J.L. Hutchison, O.M. Zhigalina, V.V. Artemov, Y.V. Grigoriev, K.R. Izrael'yants, S.G. L'vov. Influence of electric field and emission current on the configuration of nanotubes in carbon nanotube layers. 2005, Carbon, Vol. 43, pp. 3112-3123.

66. Y.M. Wong, W.P. Kang, J.L. Davidson, B.K. Choi, W. Hofmeister, J.H. Huang. Array geometry, size and spacing effects on field emission characteristics of aligned carbon nanotubes. 2005, Diam. Rel. Mater., Vol. 14, pp. 2078-2083.

67. Y.M. Wong, S. Wei, W.P. Kang, J.L. Davidson, W. Hofmeister, J.H. Huang, Y. Cui. Carbon nanotubes field emission devices grown by thermal CVD with palladium as catalysts. 2004, Diam. Rel. Mater., Vol. 13, pp. 2105-2112.

68. S.M. Lyth, F. Oyeleye, R.J. Curry, J. Davis, S.R.P. Silva. Field emission from multiwall carbon nanotubes prepared by electrodeposition without the use of a dispersant. 2006, J. Vac. Sci. Tech. B, Vol. 24, pp. 1362-1364.

69. S.M. Jung, H.Y. Jung, J.S. Suh. Horizontally aligned carbon nanotube field emitters having a long term stability. 2007, Carbon, Vol. 42, pp. 2917-2921.

70. M.P. Siegal, P.A. Miller, P.P. Provencio, D.R. Tallant. Controlled growth of carbon nanotube films for high-current field emission. 2007, Diam. Rel. Mater., Vol. 16, pp. 1793-1798.

71. P.S. Guo, T. Chen, Y.W. Chen, Z.J. Zhang, T. Feng, L.L. Wang, L.F. Lin, Z. Sun, Z.H. Zheng. Fabrication of field emission display prototype utilizing printed carbon nanotubes/nanofibers emitters. 2008, Sol. St. Electron, Vol. 52, pp. 877-881. 
72. H.Y. Jung, S.M. Jung, J.S. Suh. Horizontally aligned single-walled carbon nanotube field emitters fabricated on vertically aligned multi-walled carbon nanotube electrode arrays. 2008, Carbon, Vol. 46, pp. 1345-1349.

73. S.J. Kyung, J.B. Park, B.J. Park, J.H. Lee, G.Y. Yeom. Improvement of electron field emission from carbon nanotubes by Ar neutral beam treatment. 2008, Carbon, Vol. 46, pp. 1316-1321.

74. J. Jiang, T. Feng, X.H. Cheng, L.J. Dai, G.B. Cao, J.H. Zhang, B.Y. Jiang, X. Wang, X.H. Liu, S.C. Zou. Growth and field emission of coiled nanotubes by plasma-enhanced chemical vapor deposition. 2006, Mater. Lett., Vol. 60, pp. 10851088.

75. W.Y. Sung, J.G. Ok, W.J. Kim, S.M. Lee, S.C.Yeon, H.Y. Lee, Y.H. Kim. Synthesis and field emission characteristics of carbon nanocoils with a high aspect ratio supported by copper micro-tips. 2007, Nanotechnology, Vol. 18, p. 245603.

76. L. Pan, T. Hayashida, M. Zhang, Y. Nakayama. Field emission properties of carbon tubule nanocoils. 2001, Jpn. J. Appl. Phys., Vol. 40, pp. L235-L237.

77. Y. Wei, C. Ciw, K.A. Dean, B.F. Coll. Stability of carbon nanotubes under electric field studies by scanning electron microscopy. 2001, Appl. Phys. Lett., Vol. 79, pp. 4527-4529.

78. A. Cao, V.P. Veedu, X. Li, Z. Yao, M.N. Ghasemi-Nejhad, P.M. Ajayan. Multifunctional brushes made from carbon nanotubes. 2005, Nat. Mater., Vol. 4, pp. 540-545.

79. S. Talapatra, S. Kar, S.K. Pal, R. Vajtai, L. Ci, P. Victor, M.M. Shaijumon, S. Kaur, O. Nalamasu, P.M. Ajayan. Direct growth of aligned carbon nanotubes on bulk metals. 2006, Nat. Nanotechnol., Vol. 1, pp. 112-116.

80. Y. Zhang, X. G. Zhang, H. L. Zhang, Z. G. Zhao, F. Li, C. Liu, H.M. Cheng. Composite Anode Material of Silicon/Graphite/Carbon Nanotubes for Li-Ion Batteries. 2006, Electrochimica Acta, Vol. 51, pp. 4994-5000.

81. C.A. Bower, O. Zhou, W. Zhu. Method for Fabrication of Patterned CNT Films. US Patent \# US 627318 B1 Aug 21, 2001.

82. Z. Chen, Q. Zhang, P. Lan, B. Zhu, T. Yu, G. Cao, D. den Engelsen. UltrahighCurrent Field Emission from Sandwich-Grown Well-Aligned Uniform Multi-Walled Carbon Nanotube Arrays with High Adherence Strength. 2007, Nanotechnology, Vol. 18, p. 265702.

83. C.Y. Choi, Z. Zheng, K.W. Wong, Z.L. Du, W.M. Lau, R.X. Du. Fabrication of Cross-Linked Multi-Walled Carbon Nanotube Coatings with Improved Adhesion 
and Intrinsic Strength by a Two-Step Synthesis: Electrochemical Deposition and Hyperthermal Proton Bombardment. 2008, Appl. Phys. A, Vol. 91, pp. 403-406.

84. Y. Qin, M. Hu. Field Emission Properties of Electrophoretic Deposition Carbon Nanotubes Film. 2009, Appl. Surf. Sci., Vol. 255, pp. 7618-7622.

85. H.-C. Su, C.-H. Chen, Y.-C. Chen, D.-J. Yao, H. Chen, Y.-C. Chang, T.-R. Ywe. Improving the Adhesion of Carbon Nanotubes to a Substrate Using Microwave Treatment. 2010, Carbon, Vol. 48, pp. 805-812.

86. T. Jeong, J. Heo, J. Lee, S. Lee, W. Kim, H. Lee, S. Park, J.M. Kim, T. Oh, C. Park, J.-B. Yoo, B. Gong, N. Lee, S.G. Yu. Improvement of Field Emission Characteristics of Carbon Nanotubes through Metal Layer Intermediation. 2005, Appl. Phys. Lett., Vol. 87, p. 063112.

87. B.J.C. Thomas, A.R. Boccaccini, M.S.P. Shaffer. Multi-Walled Carbon Nanotube Coatings Using Electrophoretic Deposition. 2005, J. Am. Cer. Soc., Vol. 88, pp. 980-982.

88. J. Cho, S. Schaab, J.A. Roether, A.R. Boccaccini. Nanostructured Carbon Nanotube/TiO2 Composite Coatings using Electrophoretic Deposition (EPD). 2008, J. Nanopart. Res., Vol. 10, pp. 99-105.

89. J.K. Radhakrishnan, P.S. Pandian, V.C. Padaki, H. Bhusan,K.U.B. Rao, J. Xie, J.K. Abraham, V.K. Varadan. Growth of Multiwalled Carbon Nanotube Arrays by Chemical Vapour Deposition over Iron Catalyst and the Effect of Growth Parameters. 2009, Appl. Surf. Sci., Vol. 255, pp. 6325-6334.

90. L. Zhu, D.W. Hess, C.P. Wong. Assembly of Fine-Pitch Carbon Nanotube Bundles for Electrical Interconnect Applications. 2007. Mater. Res. Soc. Symp. Proc.. Vol. 990. 0990-B10-01.

91. Y. Zhao, T. Tong, L. Delzeit. Interfacial Energy and Strength of MultiwalledCarbon-Nanotube-based Dry Adhesive. 2006, J. Vac. Sci. Technol. B, Vol. 24, pp. 331-335.

92. L. Qu, L. Dai, M. Stone, Z. Xia, Z.L. Wang. Carbon Nanotube Arrays with Strong Shear Binding-On and Easy Normal Lifting-Off. 2008, Science, Vol. 322, pp. 238242 .

93. A. Cao, V.P. Veedu, X. Li, Z. Yao, M.N. Ghasemi-Nejhad, P.M. Ajayan. Multifunctional Brushes Made from Carbon Nanotubes. 2005, Nat. Mater., Vol. 4, pp. 540-545.

94. S. Talapatra, S. Kar, S.K. Pal, R. Vajtai, L. Ci, P. Vicor, M.M. Shaijumon, S. Kaur, O. Nalamasu, P.M. Ajayan. Direct Growth of Aligned Carbon Nanotubes on Bulk Metals. 2006, Nat. Nanotechnol., Vol. 1, pp. 112-116. 
95. Y. Zhang, E. Suhir, Y. Xu, C. Gu. Bonding Strength of a Carbon Nanofiber Array to its Substrate. 2006, J. Mater. Res., Vol. 21, pp. 2922-2926.

96. K.M. Lee, A.A. Polycarpou. Shear Strength Determination Using the Nanoscratch Technique and Its Application to Thin Solid Films. 2006, J. Mater. Res., Vol. 21, pp. 2304-2313.

97. X. Li, B. Bhushan. A Review of Nanoindentation Continuous Stiffness Measurement Technique and Its Applications. 2002, Mater. Charact., Vol. 48, pp. 11-36.

98. M. Bedewy, E.R. Meshoy, H. Guo, E.A. Verploegen, W. Lu, A.J. Hart. Collective Mechanism of Evolution and Self-Termination of Vertically Aligned Carbon Nanotube Growth. 2009, J. Phys. Chem. C, Vol. 113, pp. 20576-20582.

99. S. Hofmann, R. Sharma, C. Ducati, G. Du, C. Mattevi, C. Cepek, M. Cantoro, S. Pisana, A. Parvez, F. Cervantes-Sodi, A.C. Ferrari, R. Dunin-Borkowski, S. Lizzit, L. Petaccia, A. Goldoni, J. Robertson. In Situ Observations of Catalyst Dynamics during Surface-Bound Carbon Nanotube Nucleation. 2007, Nano Lett., Vol. 7, pp. 602-608.

100. E.R. Meshot, A.J. Hart. Abrupt Self-Termination of Vertically-Aligned Carbon Nanotube Growth. 2008, Appl. Phys. Lett., Vol. 92, p. 113107.

101. . S. Pisana, M. Cantoro, A. Parvex, S. Holfmann, A.C. Ferrari, J. Robertson. The role of precursor gases on the surface restructuring of catalyst films during carbon nanotube growth2007, Phys. E, Vol. 37, pp. 1-5.

102. J. March, Advanced Organic Chemistry: Reactions, Mechanisms, and Structure. Wiley, New York (USA), 1992. pp. 23-25.

103. I.C. Chen, L.-H. Chen, C. Orme, A. Quist, R. Lal, S. Jin. Fabrication of highaspect-ratio carbon nanocone probes by electron beam induced deposition patterning. 2006, Nanotechnology, Vol. 17, pp. 4322-4326.

104. J.F. Shackelford, W. Alexander. Thermal properties of materials. CRC Materials Science and Engineering Handbook. Third edition. CRC Press LLC, Boca Raton (USA), 2001, Table 104.

105. R.G. Munro. Evaluated material properties for a sintered alpha-Al2O3. 1997, J. Am. Ceram. Soc., Vol. 80, pp. 1919-1928.

106. J.F. Shackelford, W. Alexander. Electrical properties of materials. CRC Materials Science and Engineering Handbook. Third edition. CRC Press LLC, Boca Raton (USA), 2001, Table 291.

107. W. Zhu, C. Bower, O. Zhou, G. Kochanski, S. Jin. Large current density from carbon nanotube field emitters. 1999, Appl. Phys. Lett. , Vol. 75, pp. 873-875. 
108. P. Xue, K.H. Park, X.M. Tao, W. Chen, X.Y. Cheng. Electrically conductive yarns based on PVA/carbon nanotubes. 2007, Composite Structures, Vol. 78, pp. 271277.

109. B. E. Kilbride, J. N. Coleman, J. Fraysse, P. Fournet, M. Cadek, A. Drury, S. Hutzler, S. Roth, W. J. Blau. Experimental observation of scaling laws for alternating current and direct current conductivity in polymer-carbon nanotube composite thin films. 2002, J. Appl. Phys., Vol. 92, pp. 4024-4030.

110. P. Miaudet, C. Bartholome, A. Derré, M. Maugey, G. Sigaud, C. Zakri, P. Poulin. Thermo-electrical properties of PVA-nanotube composite fibers. 2007, Polymer, Vol. 48, pp. 4068-4074.

111. P. Ghosh, M.Z. Yusop, S. Satoh, M. Subramanian, A. Hayashi, Y. Hayashi, M. Tanemura. Transparent and flexible field electron emitters based on the conical nanocarbon structures. 2010, J. Am. Chem. Soc., Vol. 132, pp. 4034-4035.

112. Z.-H. Yang, H.-Q. Wu. Electrochemical Intercalation of Lithium into Raw Carbon Nanotubes. 2001, Mater Chem. Phys., Vol. 71, pp. 7-11.

113. E. Frackowiak, F. Béguin. Electrochemical Storage of Energy in Carbon Nanotubes and Nanostructured Carbons. 2002, Carbon, Vol. 40, pp. 1775-1787.

114. H.-C. Shin, M. Liu, B. Sadanadan, A. M. Rao. Electrochemical Insertion of Lithium into Multi-Walled Carbon Nanotubes Prepared by Catalytic Decomposition. 2002, J. Power Sources, Vol. 112, pp. 216-221.

115. T. Ishihara, M. Nakasu, M. Yoshio, H. Nishiguchi, Y. Takita. Carbon Nanotube Coating Silicon Doped with Cr as a High Capacity Anode. 2005, J. Power Sources, Vol. 146, pp. 161-165.

116. W.X. Chen, J.Y. Lee, Z. Liu. Electrochemical Lithiation and De-Lithiation of Carbon Nanotube-Sn2Sb Nanocomposites. 2002, Electrochem. Comm., Vol. 4, pp. 260-265.

117. C. Masarapu, V. Subramanian, H. Zhu, B. Wei. Long-Cycle Electrochemical Behavior of Multiwall Carbon Nanotubes Synthesized on Stainless Steel in Li Ion Batteries. 2009, Adv. Func. Mater., Vol. 19, pp. 1008-1014.

118. L.-F. Cui, Y. Yang, C.-M. Hsu, Y. Cui. Carbon-Silicon Core-Shell Nanowires as High Capacity Electrode for Lithium Ion Batteries. 2009, Nano Lett., Vol. 9, pp. 3370-3374.

119. E.J. Yoo, J. Kim, E. Hosono, H.-S. Zhoi, T. Kudo, I. Honma. Large Reversible Li Storage of Graphene Nanosheet Families for Use in Rechargeable Lithium Ion Batteries. 2008, Nano Lett., Vol. 8, pp. 2277-2282. 
120. S.-M. Paek, E.-J. Yoo, I. Honma. Enhanced Cyclic Performance and Lithium Storage Capacity of SnO2/Graphene Nanoporous Electrodes with ThreeDimensionally Delaminated Flexible Structure. 2009, Nano Lett., Vol. 9, pp. 72-75.

121. N.A. Kaskhedikar, Lithium Storage in Carbon Nanostructures. J. Maier. 2009, Adv. Mater., Vol. 21, pp. 2664-2680.

122. G. Maurin, Ch. Bousquet, F. Henn, P. Bernier, R. Almairac, B. Simon. Electrochemical Intercalation of Lithium into Multiwall Carbon Nanotubes. 1999, Chem. Phys. Lett., Vol. 312, pp. 14-18.

123. L. Dupont, L. Laffont, S. Grugeon, S. Laruelle, V. Bodenez, J.M. Tarascon. Recent Developments in Transmission Electron Microscopy Techniques to the Characterization of Cycled Li-Ion Electrode Materials. 2007, ECS Trans., Vol. 3, pp. 139-153.

124. K. Nishidate, M. Hasegawa. Energetics of Lithium Ion Adsorption on Defective Carbon Nanotubes. 2005, Phys. Rev. B, Vol. 71, p. 245418.

125. B.J. Landi, M.J. Ganter, C.M. Schauerman, C.D. Cress, R.P. Raffaelle. Lithium Ion Capacity of Single Wall Carbon Nanotube Paper Electrodes. 2008, J. Phys. Chem. C, Vol. 112, pp. 7509-7515.

126. Are Bulk Defective Carbon Nanotubes Less Electrically Conducting? P.C.P. Watts, W.-K. Hsu, H.W. Kroto, D.R.M. Walton. 2003, Nano Lett., Vol. 3, pp. 549-553.

127. P.M. Parthangal, R.E. Cavicchi, M.R. Zachariah. A Generic Process of Growing Aligned Carbon Nanotubes Arrays on Metals and Metal Alloys. 2007, Nanotechnology, Vol. 18, pp. 185605(1-5).

128. Y. Matsuda, W.-Q. Deng, W.A.III. Goddard. Contact Resistance Properties between Nanotubes and Various Metals from Quantum Mechanics. 2007, J. Phys. Chem. C, Vol. 111, pp. 11113-11116.

129. J. Zhang, X. Wang, W. Yang, W. Yu, T. Feng, Q. Li, X. Liu, C. Yang. Interaction between carbon nanotubes and substrate and its implication on field emission mechanism. 2006, Carbon, Vol. 44, pp. 418-422.

130. R. Huang, X. Fan, W. Shen, J. Zhu. Carbon-Coated Silicon Nanowire Array Films for High-Performance Lithium-Ion Battery Anodes. 2009, Appl. Phys. Lett., Vol. 95, p. 133119.

131. M.-H. Park, M.G. Kim, J. Joo, K. Kim, J. Kim, S. Ahn, Y. Cui, J. Cho. Silicon Nanotube Battery Anodes. 2009, Nano Lett., Vol. 9, pp. 3844-3847. 
132. A.P. Guo, Z.W. Zhao, H.K. Liu, S.X. Dou. Electrochemical Lithiation and DeLithiation of MWNT-Sn/SnNi Nanocomposites. 2005, Carbon, Vol. 43, pp. 13921399.

133. J. Fan, T. Wang, C. Yu, B. Tu, Z. Jiang, D. Zhao. Ordered, Nanostructured TinBased Oxides/Carbon Composite as the Negative-Electrode Material for LithiumIon Batteries. 2004, Adv. Mater, Vol. 16, pp. 1432-1436.

134. J. Shu, H. Li, R. Yang, Y. Shi, X.Huang. Cage-Like Carbon Nanotubes/Si Composite as Anode Material for Lithium Ion Batteries. 2006, Electrochem. Comm., Vol. 8, pp. 51-54.

135. Y. Wang, H. C. Zeng, J. Y. Lee. Highly Reversible Lithium Storage in Porous SnO2 Nanotubes with Coaxially Grown Carbon Nanotube Overlayers. 2006, Adv. Mater., Vol. 18, pp. 645-649.

136. W.Wang, P.N. Kumta. Reversible High Capacity Nanocomposite Anodes of Si/C/SWNTs for Rechargeable Li-Ion Batteries. 2007, J. Power Sources, Vol. 172, pp. 650-658.

137. M.S. Park, S.A. Needham, G.-X. Wang, Y.-M. Kang, J.-S. Park, S.-X. Dou, H.-K. Liu. Nanostructured SnSb/Carbon Nanotube Composites Synthesized by Reductive Precipitation for Lithium-Ion Batteries. 2007, Chem. Mater., Vol. 19, pp. 24062410.

138. C. Li, N.Sun, J. Ni, J. Wang, H. Chu, H. Zhou, M. Li. Y. Li. Controllable Preparation and Properties of Composite Materials Based on Ceria Nanoparticles and Carbon Nanotubes. 2008, J. Solid State Chem., Vol. 181, pp. 2620-2625.

139. S.-F. Zheng, J.-S. Hu, L.-S. Zhong, W.-G. Song, L.-J. Wan, Y.-G. Guo. Introducing Dual Functional CNT Networks into CuO Nano/Micro Spheres toward Superior Electrode Materials for Lithium-Ion Batteries. 2008, Chem. Mater., Vol. 20, pp. 3617-3622.

140. M. Wang, Z.H. Li, G.T. Wu. Electrochemical lithium insertion properties of carbon nanotubes produced by catalytic pyrolysis of acetylene. 2005, Russ. J. Electrochem., Vol. 41, pp. 946-949.

141. J. Chen, J.Z. Wang, A.I. Minett, Y. Liu, C. Lynam, H. Liu, G.G. Wallace. Carbon nanotube network modified carbon fibre paper for Li-ion batteries. 2009, Energy Environ. Sci., Vol. 2, pp. 393-396.

142. Y. NuLi, J. Yang, M. Jiang. Synthesis and characterization of Sb/CNT and Bi/CNT composites as anode materials for lithium-ion batteries. 2008, Mater. Lett., Vol. 62, pp. 2092-2095. 
143. D. Wang, D. Choi, J. Li, Z. Yang, Z. Nie, R. Kou, D. Hu, C. Wang, L.V. Saraf, J. Zhang, I.A. Aksay, J. Liu. Self-Assembled TiO2-Graphene Hybrid Nanostructures for Enhanced Li-Ion Insertion. 2009, ACS Nano, Vol. 3, pp. 907-914.

144. C. Menachem, E. Peled, L. Burstein, Y. Rosenberg. Characterization of modified NG7 graphite as an improved anode for lithium-ion batteries. 1997, J. Power Sources, Vol. 68, pp. 277-282.

145. I.R.M. Kottegoda, Y. Kadoma, H. Ikuta, Y. Uchimoto, M. Wakihara. High-ratecapable lithium-ion battery based on surface-modified natural graphite anode and substituted spinel cathode for hybrid electric vehicles. 2005, J. Electrochem. Soc., Vol. 152, pp. A1595-A1599.

146. I.R.M. Kottegoda, Y. Kadoma, H. Ikuta, Y. Uchimoto, M. Wakihara. Enhancement of rate capability in graphite anode by surface modification with zirconia. 2002, Electrochem. Solid-State Lett., Vol. 5, pp. A275-A278.

147. Y.S. Jung, A.S. Cavanagh, L.A. Riley, S.-H.Kang, A.C. Dillon, M.D. Groner, S.M. George, S.-H. Lee. Ultrathin direct atomic layer deposition on composite electrodes for highly durable and safe Li-ion batteries. 2010, Adv. Mater., Vol. 22, pp. 21722176.

148. D. Aurbach, Y. Ein-Eli, B. Markovsky, A. Zaban, S. Luski, Y. Carmeli, H. Yamin. The study of electrolyte solutions based on ethylene and diethyl carbonates for rechargeable Li batteries. 1995, J. Electrochem. Soc., Vol. 142, pp. 2882-2890.

149. K. Xu, Y. Lam, S.S. Zhang, T.R. Jow, T.B. Curtis. Solvation sheath of Li+ in nonaqueous electrolytes and its implication of graphitelelectrolyte interface chemistry. 2007, J. Phys. Chem. C, Vol. 111, pp. 7411-7421.

150. H. Yang, H. Bang, K. Amine, J. Prakash. Investigations of the exothermic reactions of natural graphite anode for Li-ion batteries during thermal runaway. 2005, J. Electrochem. Soc., Vol. 152, pp. A73-A79.

151. T. Doi, L. Zhao, M. Zhou, S. Okada, J. Yamaki. Quantitative studies on the thermal stability of the interface between graphite electrode and electrolyte. 2008, J. Power Sources, Vol. 185, pp. 13380-1385.

152. M. Ritala, M. Leskela. Handbook of Thin Film Materials. Academic Press, San Diego (USA), 2001.

153. Y.-S. Min, E.J. Bae, K.S. Jeong, Y.J. Cho, J.-H. Lee, W.B. Choi, G.-S. Park. Ruthenium oxide nanotube arrays fabricated by atomic layer deposition using a carbon nanotube template. 2003, Adv. Mater., Vol. 15, pp. 1019-1022.

154. S. Choi, J. Koo, H. Jeon, Y. Kim. Plasma-enhanced atomic-layer deposition of a HfO2 gate dielectric. 2004, J. Korean Phys. Soc., Vol. 44, pp. 35-38. 
155. J.Y. Kim, D.Y. Kim, H.O. Park, H. Jeon. Characteristics and compositional variation of TiN films deposited by remote PEALD on contact holes. 2005, J. Electrochem. Soc., Vol. 152, pp. G29-G34.

156. S. Kim, S. Woo, H. Hong, H. Kim, H. Jeon, C. Bae. Effect of buffer layer for HfO2 gate dielectrics grown by remote plasma atomic layer deposition. 2007, J. Electrochem. Soc., Vol. 154, pp. H97-H101.

157. T. Brousse, D. Defives, L. Pasquereau, S.M. Lee, U. Herterich, D.M. Schleich. Metal oxide anodes for Li-ion batteries. 1997, Ionics, Vol. 3, pp. 332-337.

158. S.S. Zhang, T.R. Jow. Study of Poly (Acrylonitrile-Methyl Methacrylate) as Binder for Graphite Anode and LiMn2O4 Cathode of Li-Ion Batteries. 2002, J. Power Sources, Vol. 109, pp. 422-426.

159. N. Du, H. Zhang, B.D. Chen, J.B. Wu, X.Y. Ma, Z.H. Liu, Y. Zhang, D. Yang, X. Huang, J. Tu. Porous Co3O4 nanotubes derived from Co4(CO)12 clusters on carbon nanotube templates: a highly efficient material for Li-battery applications. 2007, Adv. Mater., Vol. 19, pp. 4505-4509.

160. G. Chen, Z. Wang, D. Xia. One-Pot Synthesis of Carbon Nanotube@SnO2-Au Coaxial Nanocable for Lithium-Ion Batteries with High Rate Capability. 2008, Chem. Mater., Vol. 20, pp. 6951-6956.

161. Y. Fu, R. Ma, Y. Shu, Z. Cao, X. Ma. Preparation and characterization of SnO2/carbon nanotube composite for lithium ion battery applications. 2009, Mater. Lett., Vol. 63, pp. 1946-1948.

162. J. Liu, D. Xu, L. Liu. Double-shelled nanocapsules of V2O5-based composites as high-performance anode and cathode materials for Li ion batteries. 2009, J. Am. Chem. Soc., Vol. 131, pp. 12086-12087.

163. S. Venkatachalam, H. Zhu, C. Masarapu, K.H. Hung, Z. Liu, K. Suenaga, B. Wei. In-Situ formation of sandwiched structures of nanotube/CuxOy/Cu composites for lithium battery applications. 2009, ACS Nano, Vol. 3, pp. 2177-2184.

164. Y. Yu, A. Dhanabalan, C.-H. Chen, C. Wang. Three-dimensional porous amorphous SnO2 thin films as anodes for Li-ion batteries. 2009, Electrochimica Acta, Vol. 54, pp. 7227-7230.

165. P. Limthongkul, H. Wang, Y.-M. Chiang. Nanocomposite Li-ion battery anodes produced by the partial reduction of mixed oxides. 2001, Chem. Mater., Vol. 13, pp. 2397-2402.

166. Z. Wang, M.A. Fierke, A. Stein. Porous carbon/tin (IV) oxide monoliths as anodes for lithium-ion batteries. 2008, J. Electrochem. Soc., Vol. 155, pp. A658-A663. 
167. G.X. Wang, Y. Chen, K. Konstantinov, M. Lindsay, H.K. Liu, S.X. Dou. Investigation of cobalt oxides as anode materials for Li-ion batteries. 2002, J. Power Sources, Vol. 109, pp. 142-147.

168. Z.P.Guo, Z.W.Zhao, H.K. Liu, S.X. Dou. Electrochemical lithiation and delithiation of MWNT-Sn/SnNi nanocomposites. 2005, Carbon, Vol. 43, pp. 13921399.

169. J.R. Macdonald. Impedance spectroscopy - emphasizing solid materials and systems.John Wiley \& Sons, New York (USA), 1987.

170. M.E. Orazem, B. Tribollet. Electrochemical impedance spectroscopy. John Wiley \& Sons, Hoboken (USA), 2008.

171. C.K. Chan, R. Ruffo, S.S. Hong, Y. Cui. Surface chemistry and morphology of the solid electrolyte interphase on silicon nanowire ilthium-ion battery anodes. 2009, J. Power Sources, Vol. 189, pp. 1132-1140.

172. S. Zhou, D. Wang. Unique lithiation and delithiation processes of nanostructured metal silicides. 2010, ACS Nano, Vol. 4, pp. 7014-7020.

173. Instruments, Gamry. Basics of Electrochemical Impedance Spectroscopy. Application notes - Gamry Instruments. [Online] 2009. [Cited: July 19, 2011.] http://www.gamry.com/App_Notes/EIS_Primer/Basics_Of_\%20EIS.pdf.

Application Note Rev. 1.0 9/3/2010.

174. D. Andre, M. Meiler, K. steiner, Ch. Wimmer, T. Soczka-Guth, D.U. Sauer. Characterization of high-power lithium-ion batteries by electrochemical impedance spectroscopy.I. Experimental investigation. 2011, J. Power Sources, Vol. 196, pp. 5334-5341.

175. D. Andre, M. Meiler, K. steiner, Ch. Wimmer, T. Soczka-Guth, D.U. Sauer. Characterization of high-power lithium-ion batteries by electrochemical impedance spectroscopy.II: Modelling. 2011, J. Power Sources, Vol. 196, pp. 5349-5356.

176. M. Pfanzelt, P. Kubiak, M. Fleischhammer, M. Wohlfahrt-Mehrens. TiO ${ }_{2}$ rutile-An alternative anode material for safe lithium-ion batteries. 2011, J. Power Sources, Vol. 196, pp. 6815-6821.

177. S. Leroy, F. lanchard, R. Dedryvere, B. Carre, D. Lemordant, D. Gonbeau. Surface film formation on a graphite electrode in Li-ion batteries: AFM and XPS study. 2005, Surf. Interface Anal., Vol. 37, pp. 773-781.

178. K. Edstrom, M. Herstedt, D.P. Abraham. A new look at the solid electrolyte interhase on graphite anodes in Li-ion batteries. 2006, J. Power Sources, Vol. 153, pp. 380-384. 
179. H. Bryngelsson, M. Stjerndahl, T. Gustafsson, K. Edstrom. How dynamic is the SEI? 2007, J. Power Sources, Vol. 174, pp. 970-975. 


\section{CONCLUSIONS}

This dissertation presented a detailed study on growth of multi walled carbon nanotubes (MWCNT) on interface-engineered substrates and application of such structures in enhancing performance of two types of CNT based devices - field emission device and lithium ion battery. While complete structural characterization and field emission analysis have been performed for the field emission devices, application of CNT based anodes in Li-ion batteries was supported by electrochemical and structural characterization, with special emphasis given on understanding the stability of solid electrolyte interphase (SEI), which is very closely related to reliable performance and safety of the battery. It was observed in the present study that the interface-controlled growth of CNTs significantly affected the emission properties of field emitters. Furthermore, application of similar structures as anodes of Li-ion batteries improved the capacity of anode by a factor of three, clearly indicating the beneficial effects of this structure. The main aim of the present study was to develop CNT based energy efficient devices, which was achieved by successful demonstration of these two types of devices. Specific conclusions on this study can be summarized as follows:

Substrate for MWCNT growth has been observed to have profound effect on the field emission properties. Among the substrates studied, i.e., $\mathrm{Cu}, \mathrm{Si}, \mathrm{W}, \mathrm{Al}$ and LTCC, it was observed that $\mathrm{Cu}$ substrate offered best field emission response, in terms of lower turn-on field, high level of emission current and good stability during long-time operation. This behavior can be related to several favorable factors such as strong bonding between CNTs and substrate, higher electrical and 
thermal conductivities of $\mathrm{Cu}$ compared to others, which led to easier electron injection and faster heat dissipation, respectively. While low resistance to electron injection facilitated lower turn-on field, high heat dissipation ensured that the substrate-CNT bonding was not damaged, which led to better long-time operation.

Ti appeared as a good interface layer, which enhanced bonding between $\mathrm{Cu}$ and CNTs. Further, its low resistance ensured that electron could be transferred through this layer without much hindrance.

$\mathrm{A} \mathrm{Cu}$ interface layer - for non-Cu substrates, acted as a source of electrons and easy flow path for electrons and thus, increased field emission response of MWCNTs grown on substrates like Si, W, Al, LTCC. This observation can lead to achievement of high emission current, even in cases where $\mathrm{Cu}$ can not be directly used as substrate for device design consideration.

Catalyst used for CNT growth also affected field emission behavior, as it was present in the electron flow path, either at root or tip of the CNTs. Ni catalyst was found to outperform Fe catalyst, in consideration of turn-on field.

MWCNTs were found to bond strongly with $\mathrm{Cu}-\mathrm{Ti}$ (substrate-interlayer combination) than with Si-Ti combination. Nano-scrtaches performed on both these types of samples showed almost 4.7 times higher bonding energy for $\mathrm{Cu}$ and $\mathrm{CNT}$, as compared to $\mathrm{Si}$ and CNT. This huge difference can be directly related to 
intermetallic formation between $\mathrm{Cu}$ and $\mathrm{Ti}$ and $\mathrm{TiC}$ formation between $\mathrm{Ti}$ and $\mathrm{C}$. However, Si did not react with Ti to form any such intermetallic.

Nano-scratch technique offered high accuracy and resolution, such that it can even be applied to quantify bonding energy of individual (lithographically patterned) carbon nanocones. This technique can be used for any kind of onedimensional nanomaterial to quantify its bonding with substrate materials. However, caution should be exercised to explain the results obtained by nanoscratch as bonding energy is strongly dependent on material's history.

3-dimensional (3-D) emitter, which constituted of MWCNTs grown within microchannels engraved in $\mathrm{Cu}$ substrates, showed much better performance as compared to conventional 2-D emitters. 3-D CNT emitter on $\mathrm{Cu}$ substrate was found offer very high emission current density (189 mA/cm², RMS), which was one of the best emission currents, reported in open literature. Emission current was found to be directly related with number of micro-channels up to the level of 16 channels, experimented during this study.

3-D emitter structure on $\mathrm{Cu}$ substrate with 16 channels showed a gigantic 27 times enhancement in current density, as compared to its 2-D counterpart. It also offered a huge 23 times increment in emission current density over similar 3-D structure on alumina substrate. 
Graphene-CNT hybrid emitter offered two extremely important features to field emitters - transparency and flexibility, thus making it suitable for next generation flexible display materials. This hybrid emitter offered sufficient emission performance to establish feasibility of the proof-of-concept device.

MWCNT-on-Cu was used as anode of Li-ion batteries and the anode performed excellently. Even at very high charging/discharging rate of $1.116 \mathrm{Ag}^{-1}(3.0 \mathrm{C})$, the electrode showed a reversible capacity of $767 \mathrm{mAhg}^{-1}$, representing $106 \%$ increment in capacity as compared to the theoretical capacity of graphite anodes $\left(372 \mathrm{mAhg}^{-1}\right)$.

MWCNT-on- $\mathrm{Cu}$ anode also offered excellent stability of its specific capacity, up to 50 cycles. The structure showed zero capacity loss, after few initial cycles, which made it attractive for application in Li-ion batteries. Such outstanding performance of the anode could be related with CNTs' high conductivity, surface area and zero expansion/contraction during lithiation/de-lithiation and strong bonding between $\mathrm{CNTs}$ and $\mathrm{Cu}$ substrate.

An ultra-thin alumina coating of the MWCNTs (through atomic layer deposition process) further enhanced specific capacity of the anodes and added extra safety feature to it. Specific capacity increment can be related to extra $\mathrm{Li}^{+}$ion intercalation sites offered by alumina. 
$>$ Differential scanning calorimetry (DSC) studies showed no appreciable exothermic peak for the MWCNT anode, which supported its safety issue.

$>$ Electrochemical impedance spectroscopy (EIS) and x-ray photoelectron spectroscopy (XPS) explained reactions happening in solid electrolyte interphase (SEI) and low impedance of the cell during long run, which clarified excellent stability of the electrode. 


\section{RECOMMENDATIONS FOR FUTURE RESEARCH}

The current research was aimed to develop energy efficient devices based on carbon nanotubes. In that effort, highly efficient 2-dimensional and 3-dimensional CNT based field emitters have been demonstrated. Application of MWCNT-on-Cu in Li-ion batteries (as anode) offered much higher specific capacity and excellent capacity retention ability. With these success stories, the aim of the research was achieved. However, 'science' never stops and there is no end to scientific understanding and technological developments. Some of the related issues need further exploration to finally achieve the goal of commercial production of these CNT based devices. Following is a list of recommendations for advancing this research towards its final destination.

\section{Interface engineering: Optimization of structure}

In the present study, only five substrate materials, two interfacial layers and two catalysts could be studied. However, this set of experiments should be expanded to cover more substrate-interface-catalyst combinations with an aim to optimize the structure required for best field emission performance of CNT based emitters. Further, addition of $\mathrm{Cu}$ intermediate layer should also be extended to all possible substrate materials, so that the degree of field enhancement is known, when CNTs are grown on all such substrates. On a large scale, this study can lead to creation of a roadmap for optimized performance of CNT based field emitters. This important step will certainly help to popularize application of CNT field emitters. 


\section{3-D field emitters: Simulation and experimentation}

The present study portrays enormous scope for 3-D field emitters, which offers very high emission current density. Many scientific issues are not yet fully understood for the 3-D field emitters. For example, knowledge about shape and magnitude of electric fields of such emitters can assist to understand interaction between different electric fields and thus, can predict maximum possible emission current. Simulation studies can be helpful to understand these issues very quickly. In parallel, experiments should also be performed to practically measure highest emission current achievable from such devices. This knowledge will be extremely useful in expanding the application areas of 3-D field emitters.

\section{Performance optimization of CNT-based anodes in Li-ion batteries}

This study has shown good promise for application of directly grown CNTs on $\mathrm{Cu}$ current collectors as anode in Li-ion batteries. However, before this structure can be used commercially further characterization is required. These anodes are to be tested for longer cycles, may be for 1000 cycles, their rate capabilities are needed to be understood more clearly - specifically at very higher current rates. Further, all the characterization should be repeated at elevated and lower temperatures, in order to understand their behavior at extreme weather conditions. With all these information in hand, it will be easy to perform full-cell tests with commercially available or promising cathode materials, which will finally lead to probable commercialization of these anodes. 


\section{Understanding the mechanism of Li-ion intercalation/de-intercalation}

Though XPS and EIS studies have highlighted some of the important issues related to the mechanism of charge transfer in MWCNT based batteries, further scientific studies are required to be performed in order to understand the system fully. This involves thorough XPS and EIS studies at different states of charge and depths of discharge, for many numbers of cycles. Further, an in-situ TEM study can reveal more information about structural change of CNTs at nano-level during charging and discharging. All these basic information will be helpful in further developing the system.

\section{Graphene-CNT hybrid structure as anode in Li-ion batteries}

The MWCNT anode structure, studied in this research, is grown directly on $\mathrm{Cu}$ current collectors. At the root of the CNTs, a few layer graphene could be inserted. While the graphene structure will offer extra intercalation sites, thus increasing capacity of the electrode, it will also offer stronger bond with the substrate, which will ensure long electrode life-time. This hybrid structure has good promise for anode application in Liion batteries.

\section{3-D electrode design and performance optimization}

In line with the idea of construction of 3-D field emitters and their excellent response, 3-D anode should be designed for Li-ion battery application also. Since, 3-D electrodes offer much more surface area, it is expected that those anodes will offer much higher specific capacities. Such 3-D electrodes should be characterized thoroughly, as is 
done in the present study and as recommended in the above-mentioned points (\# 3 and 4) to gain full knowledge about such electrodes. 


\section{APPENDICES}

\section{Appendix-1: Growth time effect on nano-scratch tests}
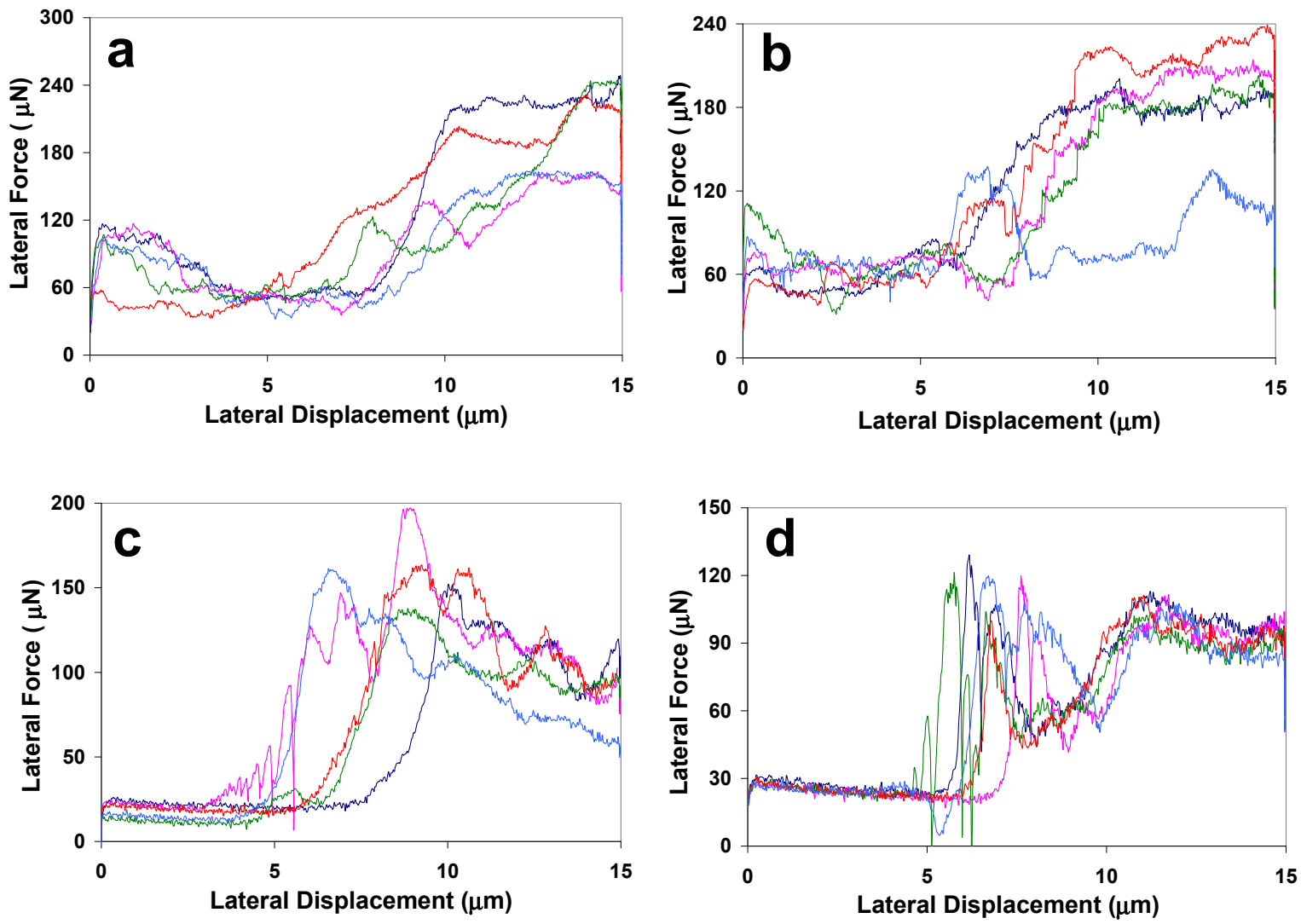

Figure A1: Nano-scratch tests on Cu ( $a$ and b) and Si (c and d) substrates, after a CNT growth time of 2 minutes ( $a$ and $c$ ) and 30 minutes ( $b$ and $d$ ). Both the samples have shown approximately same lateral force increment values for 2 minutes and 30 minutes, indicating that length of CNTs (which is much higher for 30 minutes samples) do not affect the scratching force to any significant level. 


\section{Appendix-2: Calculation of de-bonding energy of single CNTs from nano-scratch} tests

For calculation of debonding energy, following relations were used.

$$
\begin{aligned}
& A=W \times L \\
& W=8 \times h \\
& E=F \times L \\
& N=\rho \times A \\
& E^{0}=E / N
\end{aligned}
$$

Where,

$$
\begin{aligned}
& \mathrm{A}=\text { Area of interest } \mu \mathrm{m}^{2} \\
& \mathrm{~W}=\text { Width of scratch, } \mu \mathrm{m} \\
& \mathrm{L}=\text { Arbitrary length of scratch, taken as } 1 \mu \mathrm{m} \\
& \mathrm{h}=\text { Normal displacement as measured from the normal displacement plots generated by } \\
& \quad \text { the software, } \mathrm{nm} \\
& \mathrm{E}=\text { Energy to de-bond CNTs, } \mathrm{pJ} \\
& \mathrm{F}=\text { Lateral force as measured from lateral force-displacement plots generated by the } \\
& \quad \text { software, } \mu \mathrm{N} \\
& \mathrm{N}=\text { Number of CNTs in area of interest A } \\
& \rho=\text { CNT density (as measured from SEM images), } / \mu \mathrm{m}^{2} \\
& \mathrm{E}^{0}=\text { Energy required to de-bond each CNT, pJ per CNT }
\end{aligned}
$$

Lateral force $(F)$ used in this calculation were calibrated to subtract the effects of substrates and catalysts. 
During CNT density calculations, 50 images (which is a statistically significant number) were captured at high magnification $(>30,000 \mathrm{X})$ for each type of samples. Number of CNTs in the area of images was counted on each of these images using ImageJ software. ${ }^{37}$ For CNT density calculation, counted number for CNTs for each image was divided by the area of the image. 


\section{Appendix-3: Calculation of area in 3-D field emitters}

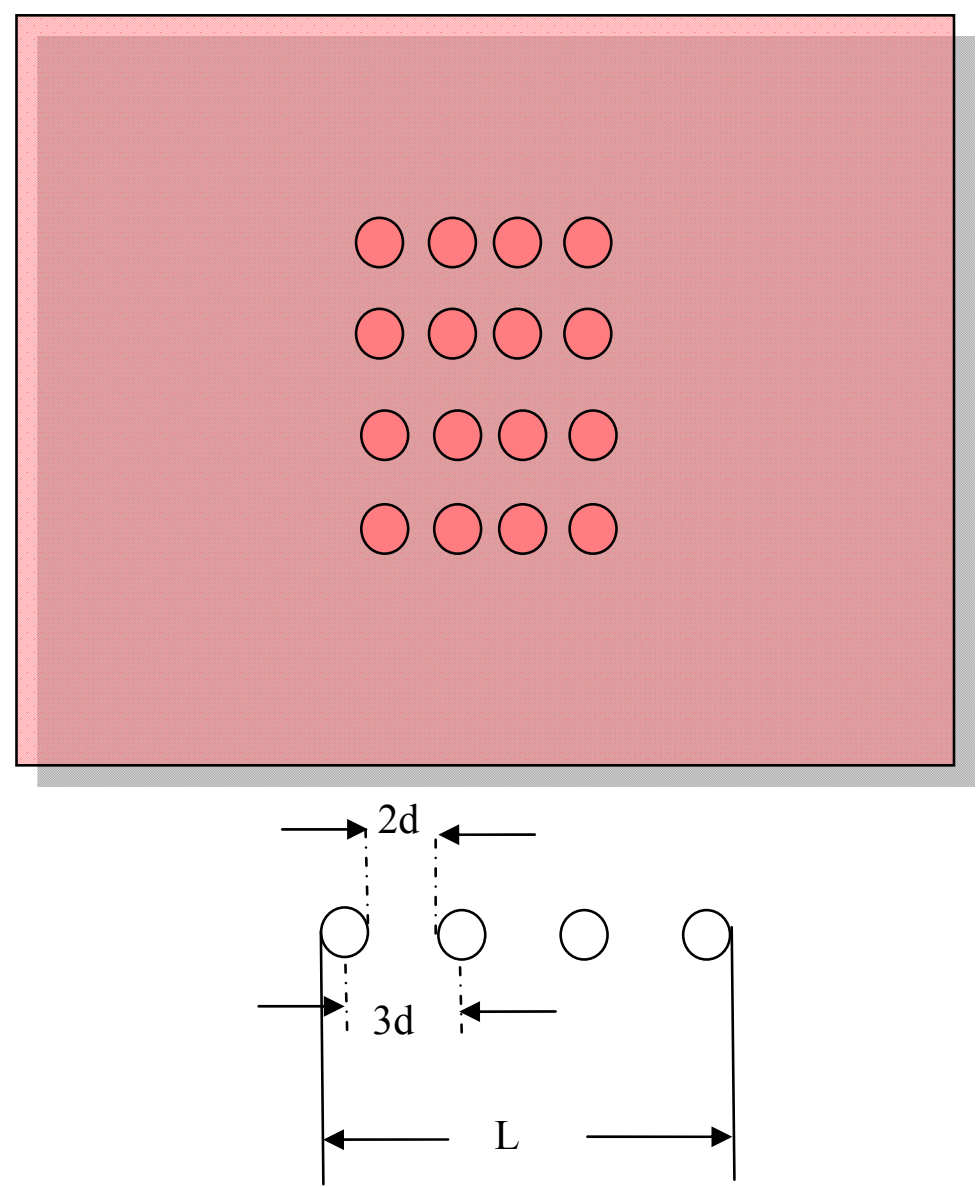

Figure A2: Schematic representation of calculation scheme followed for MCP area enhancement.

Let, $d=$ diameter of each channel $(\sim 100 \mu \mathrm{m}) ; \mathrm{t}=$ thickness of copper $\mathrm{MCP}(\sim 250 \mu \mathrm{m})$.

If number of channels in one line (of length $\mathrm{L}$, taken as $1 \mathrm{~mm}$ in the present study) is given by $\mathrm{n}$, then the following relation holds true for the geometry given above.

$\mathrm{L}=(\mathrm{n}-1) \cdot 3 \mathrm{~d}+2 \cdot(\mathrm{d} / 2)$

So, $n=\{(L-d) / 3 d+1\}$ 
In a square of size $\mathrm{L}^{2}$ (taken as $1 \mathrm{~mm}^{2}$ in the present study), there will be $\mathrm{n}^{2}$ number of channels. In the calculation (excel file), we assumed $n^{2}=N$.

Now, the total 3-dimensional area of the MCPs will be given by A.

$\mathrm{A}=\left[(\mathrm{N} . \pi \mathrm{dt})+\left\{\mathrm{L}^{2}-\left(\mathrm{N} . \pi \mathrm{d}^{2} / 4\right)\right\}\right]$

$\mathrm{A}_{0}=$ Standard 2-dimensional emitter area $=1 \mathrm{~mm}^{2}$

Table A1: Active area for CNT growth in each sample (calculated following the method mentioned above)

\begin{tabular}{|c|c|}
\hline Number of channels & $\begin{array}{c}\text { Active area for CNT } \\
\text { growth }\left(\mathbf{c m}^{\mathbf{2}} \mathbf{)}\right.\end{array}$ \\
\hline 4 & 0.012826 \\
\hline 9 & 0.016359 \\
\hline 16 & 0.021304 \\
\hline
\end{tabular}




\section{Appendix-4: Characteristics of graphene used in transparent, flexible field emitter}
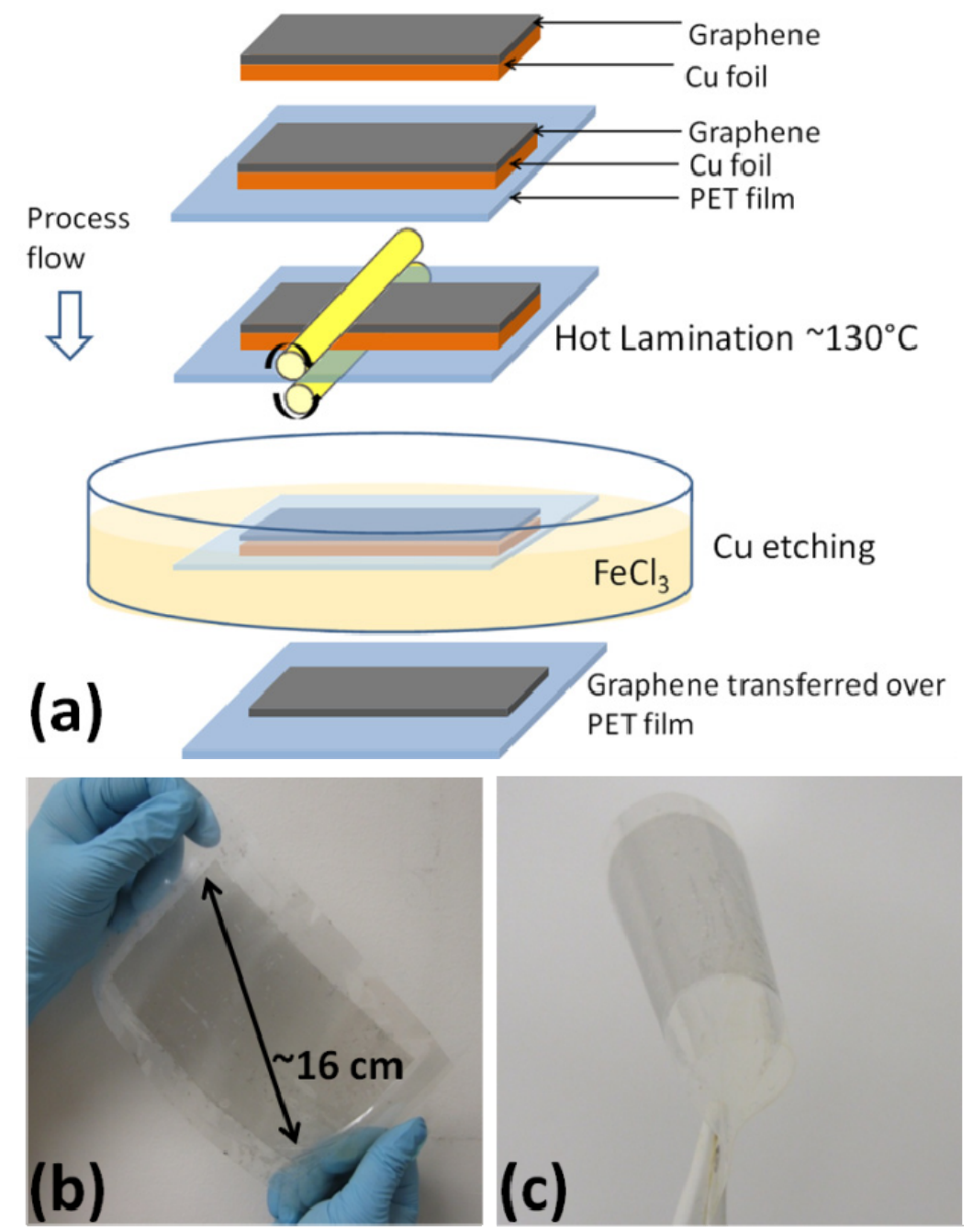

Figure A3: Fabricating graphene-on-PET. (a) Process flow for graphene transfer from Cu foil to PET substrate. Hot press lamination and chemical etching processes were used in this method. (b) Large area graphene film transferred over PET substrate. (c) Flexibility of graphene/PET film. 

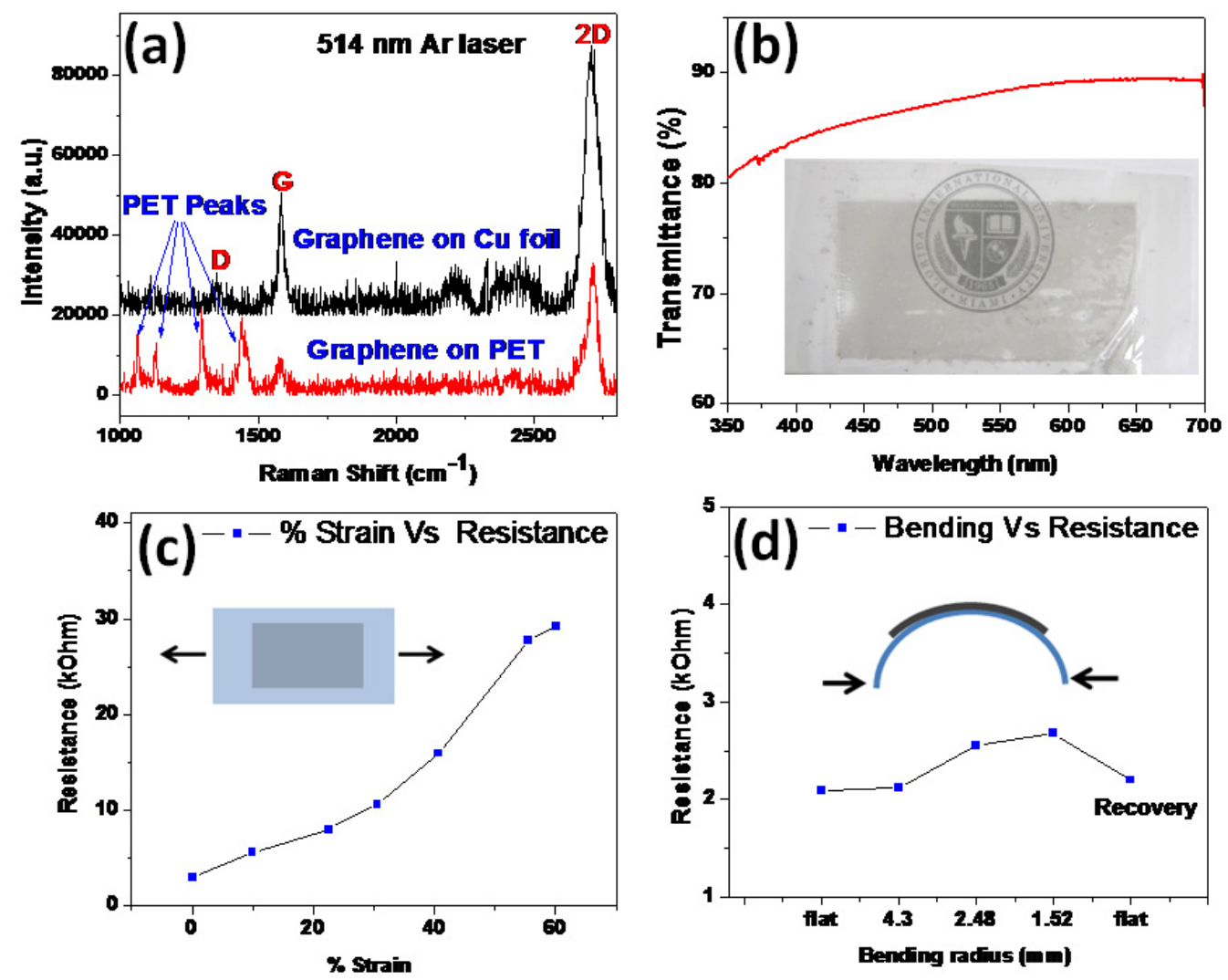

Figure A4: Characteristics of graphene-on-PET. (a) Raman spectra from graphene on copper foil and PET substrates. (b) Transmittance of graphene film over PET substrate. Inset shows large area transparent graphene/PET film. (c) Variation in resistance of graphene/PET film uniaxial stretched by $60 \%$. (d) Resistance of graphene/PET film with different bending radii. Insets show schematic of stress modes applied to graphene/PET film.

\section{Reference}

1. Ved Prakash Verma, Santanu Das, Indranil Lahiri, Wonbong Choi, Large-area graphene on polymer film for flexible and transparent anode in field emission device, Applied Physics Letters, 96 (20), 2010, 203108 (1-3). 


\section{Appendix-5: List of publications during current PhD research}

\section{$\underline{\text { Patent }}$}

1. W.B. Choi, S.J. Cho, I. Lahiri, High efficiency lithium ion battery anode using interface-controlled binder-free carbon nanotubes grown on metal/alloy substrates, US Provisional Patent Appl. No. 61/222,481 (Filed on July 2, 2009).

\section{$\underline{\text { Book Chapters }}$}

1. Indranil Lahiri, Wonbong Choi, Field emission and graphene: An overview of current status, In "Graphene: Synthesis and applications" Ed. W. Choi, J.-W. Lee, CRC Press (Boca Raton, USA), August 2011 (expected publication date), ISBN: 9781439861875.

2. Indranil Lahiri, Wonbong Choi, Graphene and graphene based materials in solar cell application, In "Graphene: Synthesis and applications" Ed. W. Choi, J.-W. Lee, CRC Press (Boca Raton, USA), August 2011 (expected publication date), ISBN: 9781439861875.

\section{Journals (Published/Accepted)}

1. Indranil Lahiri, Seung-Min Oh, Jun Y. Hwang, Chiwon Kang, Hyeongtag Jeon, Rajarshi Banerjee, Yang-Kook Sun, Wonbong Choi, Ultrathin alumina coated carbon nanotubes as negative electrodes for high capacity and safe Li-ion battery, Journal of Materials Chemistry (DOI: 10.1039/C1JM11474C). (Impact Factor: 5.099) 
2. Indranil Lahiri, Wonbong Choi, Interface control: A modified rooting technique for enhancing field emission from multiwall carbon nanotube based bulk emitters, Acta Materialia, 59, 2011, 5411-5421. (Impact Factor: 3.781)

3. Indranil Lahiri*, Debrupa Lahiri*, Sungho Jin, Arvind Agarwal, Wonbong Choi, Carbon Nanotubes: How strong is their bond with the substrate?, ACS Nano, 5(2), 2011, 780-787. (Impact Factor: 9.855) * Authors of equal contribution

Highlighted as spotlight in "Nanowerk"

(http://www.nanowerk.com/spotlight/spotid=19707.php)

4. Indranil Lahiri, Ved Prakash Verma, Wonbong Choi, An all-graphene based transparent and flexible field emission device, Carbon, 49 (5), 2011, 1614-1619. (Impact Factor: 4.893)

5. Indranil Lahiri, Santanu Das, Chiwon Kang, Wonbong Choi, Application of carbon nanostructures - Energy to electronics, JOM, 63(6), 2011, 70-76. (Impact Factor: 1.175)

6. Santanu Das, Raghunandan Seelaboyina, Ved Verma, Indranil Lahiri, Jun Yeon Hwang, Rajarshi Banerjee, Wonbong Choi, Synthesis and Characterization of SelfOrganized Multilayered Graphene-Carbon Nanotube Hybrid Films, Journal of Materials Chemistry, 21, 2011, 7289-7295. (Impact Factor: 5.099)

7. Jun Huang, Unjeong Kim, Bei Wang, Indranil Lahiri, Eunhong Lee, Peter C. Eklund, Wonbong Choi, Controlled Growth of Single-walled Carbon Nanotubes for Unique Nanodevices, Journal of Nanoscience and Nanotechnology, 11(1), 2011, 262-269. (Impact Factor: 1.351) 
8. Indranil Lahiri, Sung-Woo Oh, Jun Y. Hwang, Sungjin Cho, Yang K. Sun, Rajarshi Banerjee, Wonbong Choi, High capacity and excellent stability of lithium ion battery anode using interface-controlled binder-free multiwall carbon nanotubes grown on copper, ACS Nano, 4(6), 2010, 3440-3446. (Impact Factor: 9.855)

9. Indranil Lahiri, Raghunandan Seelaboyina, Jun Y Hwang, Raj Banerjee, Wonbong Choi, Enhanced field emission from multi-walled carbon nanotubes grown on pure copper substrate, Carbon, 48 (5), 2010, 1531-1538. (Impact Factor: 4.893)

10. Indranil Lahiri, Prospects of oxide materials in Li-ion batteries, American Ceramic Society Bulletin, 89 (6), 2010, 17-18. (Impact Factor: 0.177)

11. Jun Huang, Bei Wang, Indranil Lahiri, Awnish K. Gupta, Peter C. Eklund, Wonbong Choi, Effect of bending on the resistivity and Raman spectrum of single-walled carbon nanotubes, Advanced Functional Materials, 20, 2010, 4388-4393. (Impact Factor: 8.486)

12. Ved Prakash Verma, Santanu Das, Indranil Lahiri, Wonbong Choi, Large-area graphene on polymer film for flexible and transparent anode in field emission device, Applied Physics Letters, 96 (20), 2010, 203108 (1-3). Also published in Virtual Journal of Nanoscale Science and Technology, Vol. 21, Issue 22, May 31, 2010. (Impact Factor: 3.820)

13. Raghunandan Seelaboyina, Indranil Lahiri, Wonbong Choi, Carbon Nanotube Embedded Novel 3-Dimensional Alumina Microchannel Cold Cathodes for High Electron Emission, Nanotechnology, 21 (14), 2010, 145206. (Impact Factor: 3.644) 
14. Wonbong Choi, Indranil Lahiri, Raghunandan Seelaboyina, Yong Soo Kang, Synthesis of graphene and its applications: a review, Critical Reviews in Solid State and Materials Sciences, 35, 2010, 52-71. (Impact Factor: 6.143)

\section{$\underline{\text { Peer-reviewed Symposium Proceedings (Published/Accepted) }}$}

1. Indranil Lahiri, Raghunandan Seelaboyina, Won Bong Choi, Field Emission Response from Multiwall Carbon Nanotubes Grown on Different Metallic Substrates, In Nanotubes and Related Nanostructures - 2009, Ed. Yoke Khin Yap (Mater. Res. Soc. Symp. Proc. Volume 1204, Warrendale, PA, 2010), 1204-K18-21.

2. Indranil Lahiri, Sung-Woo Oh, Yang-Kook Sun, Wonbong Choi, High specific capacity and excellent stability of interface-controlled MWCNT based anodes in lithium ion battery, Mater. Res. Soc. Symp. Proc. 2010 (accepted for publication).

3. Santanu Das, Indranil Lahiri, Chiwon Kang, Wonbong Choi, Engineering Carbon Nanomaterials for Future Applications: Energy and Bio-sensor, Proc. SPIE 8031, 80311K (2011), DOI: 10.1117/12.883743.

4. Ved P. Verma, Santanu Das, Indranil Lahiri, Wonbong Choi, Large area graphene on polymer films for transparent and flexible field emission device, Mater. Res. Soc. Symp. Proc. 2010 (accepted for publication). 


\section{$\underline{\text { Journal (Under Preparation) }}$}

1. Indranil Lahiri, Wonbong Choi, Ultra-high current density multiwall carbon nanotube field emitter structure on three-dimensional micro-channeled copper.

2. Indranil Lahiri, Wonbong Choi, Charge transfer mechanism in MWCNT anodes for lithium ion battery. 


\title{
Journal of Materials Chemistry
}

Gite this: DOt: $10.1039 / \mathrm{c} 1 \mathrm{jm} 11474 \mathrm{C}$

www.rsc.org/materials

PAPER

\section{Ultrathin alumina-coated carbon nanotubes as an anode for high capacity Li-ion batteriesं}

\author{
Indrani Lahiri, ${ }^{a}$ Seung-Min Oh, ${ }^{b}$ Jun Y. Hwang, ${ }^{c}$ Chiwon Kang, ${ }^{a}$ Mansoo Choi, ${ }^{d}$ Hyeongtag Jeon, ${ }^{d}$ \\ Rajarshi Banerjee, ${ }^{c}$ Yang-Kook Sun ${ }^{b}$ and Wonbong Choi ${ }^{\star \star b}$
}

Received 7th April 2011, Accqped 14th June 2011

DOL: $10.1039 \mathrm{cl}$ jin11474

Alumina-coated carbon nanotubes (CNTs) were synthesizal on a copper substrate and have bean used as an anode in Li-ion batteries. CNTs were grown directly on the copper current collector by chemical vapor deposition and an ultrathin layer of alumina was deposited on the CNTs by atomic byer deposition, thus forming the binder-free electrode for the Li-ion battery. While CNTs, which form the wre of thestructure, provide excellent conductivity, structural integrity and Li-ion intercalation ability, the alumini um oxide coating provides additional stabihity to the electrode, with further enhancement $o f$ apacity. The anode showed very high specific capacity, good capacity retention ability and excellent rate capability. This novd anode may be considered as an advanced anode for future Li-ion batteries.

\section{Introduction}

High demand for rediarges ble batteries in hybrid or all-eledric vehicks and portable electronicand mecical devices has anforced extensive research efforts in advanced Li-ion batteries. ${ }^{1}$ Next generation Li-ion batteries need to offer better safety as well as excellent gapacity and high rate capability. Development of new dectrode materials and novel electrode designs are, thus, bang explored for enhanding the performance of electrodes, whik maintaining their structural integrity. Mesocarbon microbead $(\mathrm{MCMB})^{2}$ and/or natural graphite are widdy used as the anode materials of commencially available Li-ion battenies. However, low thooretical spacific capacity of graphite $\left(372 \mathrm{~mA} \mathrm{~h} \mathrm{~g}^{-1}\right)$ necessitates development of new anode materials with improved capacities." Recently, Si nanostructures have recived appredable attention due to its excelknt capacity. 5 -7 Carbon nanotubes $(\mathrm{CNT} s)^{4-11}$ and various oxides ${ }^{12-4 t}$ are also highlighted as possible elatrodk mataiak.

Though initial research efforts with CNTs falled to show better apacity, ${ }^{3-10}$ we have racently demonstrated high apacity, binder-free anode using interface-controlled CNTs diratly grown on a Cu current colledor. ${ }^{11}$ Inactive metal oxides are often considered as an anode material or as one of the components of

-Deparman of Mechanioal and Mateids Englnaring. Florid

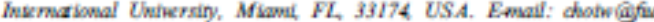

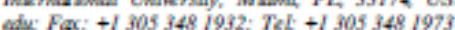

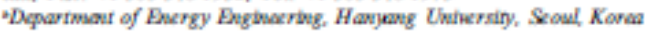

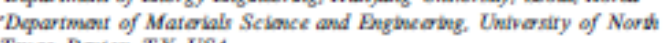
Texas, Dourow, $T X$ USA

'Departmant of Materialis Sciace and Engherabeg, Hanyang Chiversiry, Sroul Kona

† Electronic supplementary information (EST) available: Raman spectrosoopy and differential scanning calorimetry (DSC) result. See DOI: 10. $0039 \mathrm{k} / \mathrm{jml} 1474 \mathrm{c}$ anode due to their ability to protect solid dectrolyte interphase (SEI) and thus, wntributing towards better cyck life and safety. Surface modifications of active anode matariak, such as mild oxdiation, ${ }^{15}$ coating by metal oxides, ${ }^{1617}$ have been suggested for higher efficiancy electrodes. Among them, oxide-coated carbon nanostructures are expected to parform better, combining addi. tional stability, safety and capacity offered by the oxide and excellent conductivity (thus, faster charge transport) and strength (rigidity of the structure) provided by $\mathrm{C}$-nanostructures, specifically CNTs. Thus, a hybrid strudure wnsisting thinbyered oxide wated CNTs seems to be a promising candidate as an anode material for Li-ion batteries. A thin oxide costing on carbon nanotubes is expected to restrict unwanted reactions betwean carbon and the electrolyte and thus, provide extra stability and more stability to the anode. ${ }^{13}$ It may be notal at this point that solid dectrolyte intaphase (SEI), which is known to be hithium ethylene di-carbonate, ${ }^{10}$ allows Li+ ions to diffuse through it, whik blocking the electrolyte molecuks; ensuring reversible interatation and deintercalation of Li* ions during cycling ${ }^{20}$ Thus, stability of SEI is extremdy important for good cyclability of Li-ion batteries. Surface modification by oxides was observal to improve bonding of the SEI with carbon, better wetting with elatrolyte and les decomposition of SEI during charge-discharge, ${ }^{15-p}$ thus enhancing stability of the SE Improved stability of SEI also contributes towards better safety of the cell as SEI decomposition is known to be exothemnic, kading to themnal runaway. ${ }^{21}$ is Reontly, Y. Jung ef al. have danonstratal that atomic byer deposition of al umina directly on natural graphite improval stability and safety performance of the Li-ion battery." Enhanced performance, especially at elevatal temperature, was attributed to formation of a protective surface layer on graphitic particks. Therefore, introduding a protective surface byer on nanostructured carbon materials is

This joumal is $\mathbb{Q}$ The Royal Society of Chemistry 2011

1. Mater. Chem 


\title{
Interface control: A modified rooting technique for enhancing field emission from multiwall carbon nanotube based bulk emitters
}

\author{
Indranil Lahiri, Wonbong Choi ${ }^{*}$

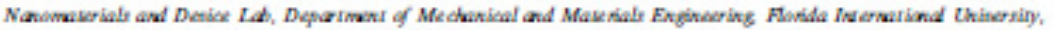

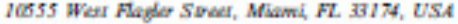 \\ Rooeivod 4 March 2011; axephod 5 May 2011 \\ Avaluble online 12 June 2011
}

Abstract

The unique properties of curbon nanotuhes (CNTs) have raised hopes that these materials might find wide application as cold cathodes in various electron sources. The excellent field amission properties shown by CNT based field emitters has further stimulated this expectation. However, efficient performance of a practical field emitter, which comprises a large number of randomly or regularly oriented CNTs, is restricted primarily due to poor junctions formed between CNTs and substrates. This study is a imed at enhancing the junction performance by way of a modified "rooting" technique interface control. In this process, the in terface between CNTs and substrate has been tailored with different metals in an attempt to improve the CNT-subrtrate junction performance. Multiwall carbon nanotubes (MWCNTs) were syn thesined on different intefface controlled substrates, i.e. Cu, Al, W, Si a nd low-temperature co-fired oeramic. All the samples produced mat-type, randomly oriented MWCNT structures. Among the four different substrates studied, MWCNT. based field emitters on Cu substrate demonstrated the best field emission response, in terms of low turn-on field, high emission current, good field enhancement fictor and excellent stahility in long-term opera bon. Emitter structures and thair field amission behavior were cor rela ted and it was shown that interface control, as a n ad vanced "rooting" process, plays an important role in determining the emission response from a bulk field emitter.

Q 2011 Acta Materinlia Inc. Puhlished by Elevier Ltd. All rights reserved.

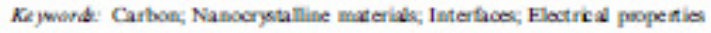

\section{Introduction}

In recent years, cold fiekd electron emitters have shown a wide range of applications, in field emission displays [1], vacuum electronic devices $[2,3]$, micro wave power amplifers [4] elect ron microscopes [5] gas sensors [6] and X-ray tubes [7], Owing to their favorable properties, namely fine tips, high aspect ratio, good chemical resistance, efficient thermal conductivity and exceptional mechanical strength, carbon nanot ubes (CNTs) have attracted most attention as the most suitable material for future field anission devices [8-12]. Many CNT-based field emission devices have shown excellent responses [13-15]. However, it was noted

\footnotetext{
- Correponding autbor. Tel: + 306343 1973 fax +1 3063481932

Email aldress choiw offin odu (W. Ooof).
}

that practical fiekd emitters, which often have a large number of vertically or randomly onental CNTs, did not perform well when compared to the emission response of a single emitter [16]. While it is feasible to manufacture a good junction between a single CNT emitter and its substrate (in most of the cases, the substrate is metalic), it is practically impossible to apply similar techniques to create good junctions between all emitters in a bulk fiekd emission device with the substrate. It is also noted that many of the reported CNT field emitters, expecially for large-seak applications, have been fabricated based on screen printing or pasting processes. The interfaces between CNTs and substrates formed in this way show structures bosely bonded either by van der Waals forcas or partial chemical bonding. Stable CNT-substrate bonding has been the focus for high efficiency field emission and for long-term stability. 


\section{Carbon Nanotubes: How Strong Is Their Bond with the Substrate?}

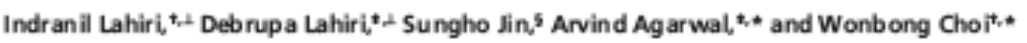

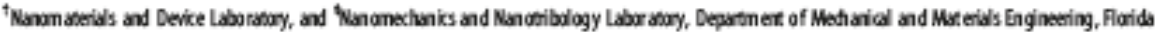

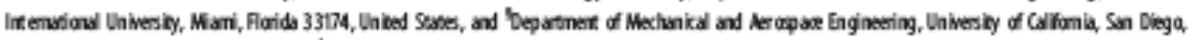

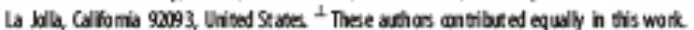

\begin{abstract}
A wate widle variety of one-dimens bna nanomaterials, such as nanotubes and nanowires, are proposed for im proved performance of a number of future devices - fromsensos to solar cells ${ }^{1,2} \mathrm{How}$ ever, desired seamiess integration, long time, reliable performance, and extended device Ifefime of all these nanomaterialsbased devices necessitates stability of the structure ${ }^{3-5}$ One of the critical issues in structural stability is the bonding between the nanomaterials and the substrate. A pocr nanomaterial-substrate adhesion may cause unteliable behavior and a very short Iffe span of such a device. Hence, knowledge about nature and strength of the nanostructure-substrate bond is considered as one of the most fundamentalissues It is of utmost importance to devise a methodology to quantify the nanomaterial-substrate bonding, to apply that technique to undestand bonding between different nanostructure-substrate bonding, ulfimately leading to development of well-bonded nanostructured devices.

Among all the one-dimensional (1-D) nanomaterials, carbon nanotubes (CNT) are probably the most important for myriad of potenfial next generation devices owing to their faschating properties, such as unique structures ${ }^{6}$ excellent eledronic and electrical properties ${ }^{7 \text { A }}$ highs secific strength good themal conductivity, ${ }^{10}$ and chemical inentness " Amost two decades of intense research activifies have resulted in the dernonstrafion of various CNT-based devices, for example, nanoelactronics, fiet emission displyys ${ }^{12,13}$ biasensars and biomateriat, ${ }^{14} 15$ and hydrogen starageand energy generstion devices ${ }^{16-19}$ Research efforts (Supporting information Table S1) to clarify the nature of bonding between nanomaterials and sub strates are thus mostly nestricted to CNTbased systems. Hawever, the qualitative methocs utiloed do not affer amy standardiaed
\end{abstract}

LAHER ET $A$.
ABSTRACT A rdiatle quafication sechrique for intorpefing nammatenid-subtrate band

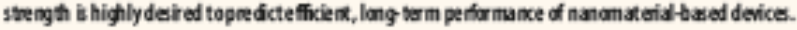
Adopting a nowd namoratrabased technique, bere we demanstrate quarifiation of carbon nanotibe (ONT)-ybatrate adheion strength for dense ON structure and for pattemed carbon nanocane (CNC) structures. Debnding erery for a inge ONT it ilustrated to range between 1 and

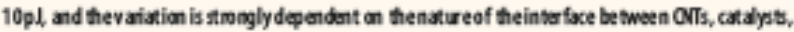
and abstrste. Our popposd techrique could be adqut for characterization of bending strength between a wide vaiety of nambibs, nanowirs, and ober onedimensional nanostruxtured materials and their undetying substrater.

KEYWORDSt nanotubes - adheslon - nanoscratch + bonding mengy

procedure and reproducible results, as the techniques are extremelyuser sensitive. The quantitative methods experimented so far have some common groumd for companson of adhesion strengtha, though all of these techniques pose several limitations. First none of these methods have been carbrated to single out the bonding between only CNT - substrate. Proposed quantitative methods do not show the amount of stress required to pull out the adhesive tape from a blank substrate (without having $\mathrm{CNT}$ grown on iff. 2418 Thus, the strength values found in these methods ane often over estimated values of exact contribution from the CNT-substrate bond. Second, the adhesion strength was calculated assuming the area of the tape as the contact area. The actual contact anea, where CNTs ate in contact with thetape, is much lawer.So, the reported values ane likely to be an under estimate of the actual strength nequired to debond $\mathrm{CNT}$ s from the substrate. Third the number of CNTs in contact with the tape was not known, and hence, none of these methods could predict adhesion strength of a single CNT. Furthermare, it was not known whether the CNTs were breaking at the CNT-substrate interface or at any defective lacations along its length or if one or few 


\title{
An all-graphene based transparent and flexible field emission device
}

\author{
Indranil Lahiri ${ }^{a}$, Ved Prakash Verma ${ }^{a}$, Wonbong Choi $a, b, *$ \\ "Nanomatsrials and Devices Labaratory, Denartment of Mechanioal and Materials Engine ering, Florida Intemational Unitursity, MA ami, \\ FL. 33174, USA \\ "WCU Departonent of Enogy Enginaring, Hanyang Uniuorsity, Sowl, Konea
}

\section{A RT ICLEINFO}

\section{Noticle kidory}

Peceived 12 Octobe 2010

Acceptad 13 December 2010

Nrallable online 21 December 2010

\begin{abstract}
A BSTRACT
All grap hene based cathode and anode structures wese fabricated and their application as a flerble and trana perent field emisision devioe ia presentad. The graphene film was grown on a $\mathrm{C}$ foal by thermal chemical vapor depoedition and laver transierred to a polymer substrate through a hot press lamination wchnique Multwall cabon nanotubes (MwCNTa) wese spinco ated onto a graphene film on a ransparent, flexoble subs tate to form the cathode of the feld emiasion device $\Lambda$ green-phosphor coatud graphene.PET film was used as the anode. The destce showed good transparency and flerbbity as well as givingan appre. dable emistion current. The simple processing techriques uad can eacly be upgraded to a large scale and be talored for any tranaparent and flerible subatrat. The device offers exdring appilicationa of carbon nanos tructures in foldable electronics.
\end{abstract}

Q 2010 Elaevier 1td. All rights reservad.

\section{Introduction}

Graphene, a new class of carbon material, has creathd novel device concepts owing to its unique m mhination of electrial, optical and mechanical properties [1,2]. This single atom thick, 2 dimenriomal (2D) material demonatrates high arrent a pocity, optical transporency and stiffness Graphene exhibits an electron mobility of $\sim 15,000 \mathrm{~cm}^{2} / \mathrm{cm}$, tra manittance -97.5\% and Young's moduhs $\sim 10 \mathrm{TPa}$ [1-4]. These properties make graphere an ideal candidate for potantial a pplication as tra naparent flexible electrode in foldable eloctronics and in new generation opte-electronic devices. Carbon nanotubes (CNT), conceptralized as 1-dimensional (1D) rolled sheets of graphene, are well known candidate for dimlay tectunologies such as field emission devices (FEDa), incandescent display and touch screens [5,6]. In particular, CNTs have huge potential in FEDe because of their high aspect ratio and low work function, along with exding mechanical properties, good chemial inertness and thermal robustness $[7,8]$. CNT bsesd
FEDs show very high field en hancement fuctors $\left(\beta \Leftrightarrow 10^{\circ}\right)$, high emiarion current and low tum on fields $(<1 \mathrm{~V} / \mathrm{m})$ [7,8]. A hybrid structure of $1 \mathrm{D} \mathrm{CNTs}$ over $2 \mathrm{D}$ graphene can be a nideal architecture for application as flevable and transparent field emisrion diplays. Application of these fleable and tranaparent diplays ranges from flevible hesd-up diplays, foldable electronics, lightring tiles and others.

Recenty, synthesis of graphene and nanowire/namotrbe hybrid structure and their application in electronic and optoelectronicsystema have been reported $[9,10]$. In a similar development, Leeet al has grown CNT forest structure byplas. ma-enhanced chemical vapor deporition (PECVD) method, on spin costed graphene film [11]. In this method CNT grows locally from patterned Fe catalyst particles on graphene film. This CNT forest/Fe-catalyst/graphene hybrid structure was transferred on poly dimethyl slowane (ommonly known as PDMS) and ured for field emistion device. However, this CNT/ graphene film ontains unwanted Fe catalyat nanoparticles, ove lapped na noplatelets of graphene in the hybrid structure.

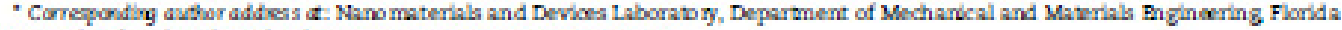
Internationd University, Mami, FI. 33174, USh. Pax +1 3053481982

E-mall add sess choi we fru adu (W. Choi).

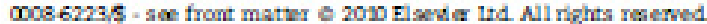

dol.101016) jeabon.2010.12044
} 
$A$ wide variety of carbon nanostructures, from zero-dimensional fullerene through one-dimensional carbon nanotube to two-dimensional graphene, have attracted attention of scientific community worldwide for their exciting properties. Canbon-based nanomaterials have found applications in a vast field -electronics, sensors, biotechnology. eneggy, structural, etc. We have concentrated our effort in doweloping now engineering nanomaterials (graphene. carbon namotubes) and their device applications in the fleld of energy generation and starage, nanoelectronics, and bio-electronic sensors. This article aims to capture those necent nesearch efforts in synthesis and applications of carbonbased nanomaterials.

\section{SYNTHESIS OF NANOMATERIALS}

\section{Graphene}

Large Scale Graphene Synthesis using Thermal Chenical Vapor Depasition

Graphene, the two-dimensional carbon nanostructure, is being considered as a promising next generation material owing to its unusual properties like high carrier concentration and mobility, good mechanical and thermal properties, extraordinary optoelectronics properties, etc. Graphene can be directly synthesized on transition metal surface (metal foil) or transition metal thin film using the thermal chemical vapor technique. Graphene research, in our lab, is concentrated on growth of graphene over a wide range of transition metals, and thin films, of size from $1 \mathrm{~cm} \times 1 \mathrm{~cm}$ up to as large as $16 \mathrm{~cm} \times 6 \mathrm{~cm}$ and their application in energy and electronics. During graphene growth, both metal foils (Ni1 and $\mathrm{Cu}$, thickness $\sim 50-200 \mu \mathrm{m}$ ) and thin films $(\mathrm{Ni}$ and $\mathrm{Cr}, 300-500 \mathrm{~nm}$ thick) were used as substrate. For the latter context, $\mathrm{Ni}$ and $\mathrm{Cu}$ thin films were deposited on $\mathrm{SiO}_{2} / \mathrm{Si}$ substrates using RF magnetron sputtering system and $\mathrm{E}$ beam evaporation. Before synthesis, the substrates were cleaned properly with acetone, methanol and iso-propanol (IPA) solution and amnealed at $1,000^{\circ} \mathrm{C}$ for $1 \mathrm{~h}$ in an inert (Ar) gas atmosphere

How would you... describe the overall significance of this paper? This article highlights syuthesis 7 af a variety of carbon namotube structunes and graphene, and discusses the widespread wee of

77 there carbon basad nomostrut in electromicos, sensor, field emission, $\checkmark$ and energy-related appolicatians. .describe this work to a

materials science and engineering professional with no experience in your techaical specialty?

- Corbon nonostruchures, especially simgie-vall and wuitt-wall carbon

I namotuber and graphens, have attracted a myriad of applications

El due to their exoiting electronici. chemicol, and mechanical

properties. An onerview of synthesis and opplications of these structure. will faclitute better understanding

E of the current stivation and promote fiture research ditrections.

E...describe this work to a layperson? One-dimensianal ourban nansotube

E and two-dimensional graphene have huge specefice surface area, excoptional electrowic and photanic

properties, axciting sensing capabilities, and are known as

wery high-strength materials.

These properties were wegul in imviting new device appications

in many fields from smaller and faster electronic gadgets to new

typer of sensors and high potentiol energy starage devioes. This article presents a summary of many swoch applications. followed by acetic acid treatment to remove any residnal oxide layer. The asprepared substrates were placed inside a quartz tube of a thermal chemical vapor deposition (CVD) system for graphene synthesis. The graphene growth process was performed at an elevated temperature of $1,000^{\circ} \mathrm{C}$ for $5-15$ minutes. The ambient of CVD furnace was kept at 0.8 atmospberic pressure in the presence of ivert gas (Ar) atmosphere and $\mathrm{CH}_{8}: \mathrm{H}_{2}$ (1:4) was used as a precursor gas mixture for grapbene growth. Finally, the funace was cooled down and brought down to room temperature under a constant flow of Ar gas in order to avoid any surface oxidation ${ }^{23}$ Graphene, grown on metallic surface, can further be transferred to polymeric substrates for device fabrication.

Fabrication of Graphene/PET

Flexible Film as Flexible Transpanent Conducting Electrodes

A unique combination of hot press lamination technique and chemical etching process is used to transfer graphene from the metal foils outo transparent flexible polyethylene tetrapthalate (PET) substrates. The transfer process is illustrated as a schematic in Figure la. Even after transferring, graphene maintains continuity in the large sample $\left(16 \times 6 \mathrm{~cm}^{2}\right.$, Figure lb). Graphene/PET film is flexible, transparent and conducting as demonstrated in Figure lc, d, and e, respectively. Furthenmore, the film exhibits $89 \%$ optical transmittance and $1.1742 \mathrm{~kW} / \mathrm{sq}$ sbeet resistance. ${ }^{2} \mathrm{Ra}-$ man spectra, taken from as-synthesized CVD graphene on $\mathrm{Cu}$ and as-transferred on PET (Figure lf), show characteristic sharp $G$ band and 2D (or G) band of graphene and a ratio of $\mathrm{I}_{\mathrm{v}} \mathrm{I}_{\mathrm{DD}} \sim 0.5$, representing monolayer grapbeve on Cu and a successful transfer of graphene on 


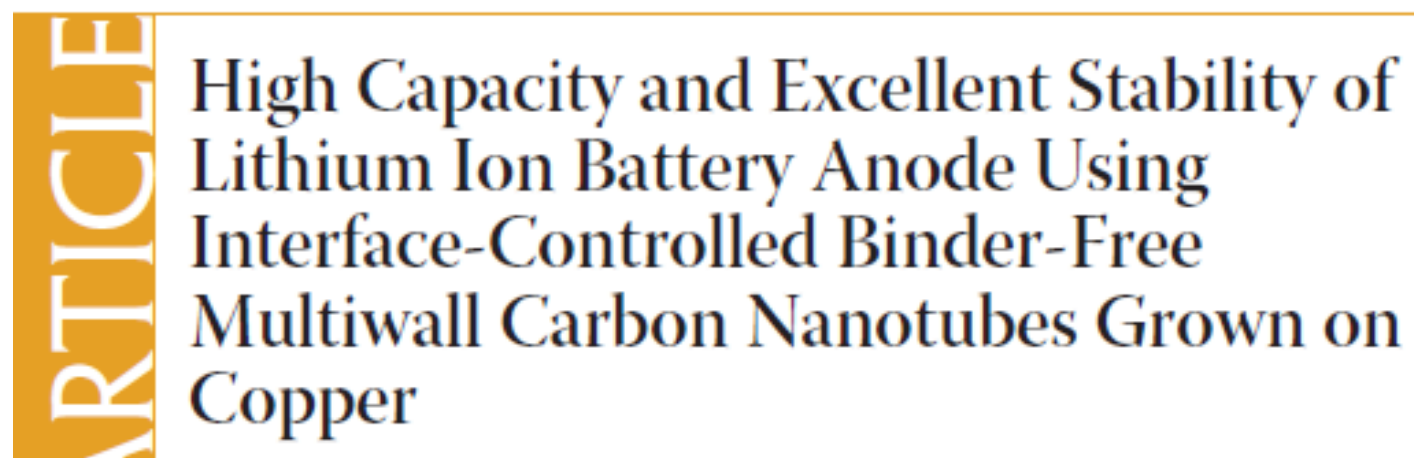

Indranll Lahirl, ${ }^{+}$Sung-Woo Oh,, Jun Y. Hwang, ${ }^{5}$ Sungjlin Cho, ${ }^{+}$Yang-Kook Sun, Rajarshl Banerjee, ${ }^{5}$ and Wonbong Chol*t,t*

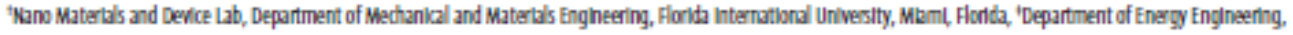

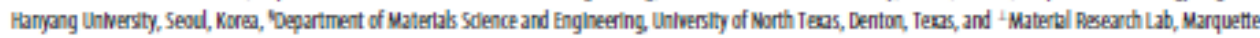
Untversily, Milwatkee, Wisconsin

\begin{abstract}
We present a nowel binder-free multiwall carbon nanoube (MWCMT) structure as an anode in Li ion batteries. The interface-controlled MwCNT structure, ggnthesined through a two-step process of atalyst deposition and chemical rapor deposition (CVD) and directly grown on a copper cuntent collector, shwed very high specific capacity, almost three times as that of graphite, eacellent rate apablity even at a charging/discharging rate of $3 \mathrm{C}$, and no capacity degradation up to 50 cydes. Sigrificantly enhanced properties of this anode could be related to high $L i$ ion intercalation on the carbon nanotube walls, strong boeding with the substrate, and excelent conductivity.

KEYWORDS: carbon nanotube - Intercalation - lithlum lon batteries - specific capacity $\cdot$ stability

Address correspo

Recelved for review Fobruary 26, 2010 and accopted Apel 28, 2010

Publlshed onlline May 4, 2010. 10.1021/nn 100400r

- 2010 Amestcan Chamical Sockty ment of new anode materials with high capadtes. The introduction of nanom (intended overall better performance simply bec. ce area as compared to their bulk co parts. Hence, much research effort was concentrated on selecting good (or the best) nanomaterlals sultable for this application.

Out of the many nanomaterlals avallable, carbon nanotubes (CNTS) attracted much attention mainly because of their excellent conductivity propertles. ${ }^{2}$ The application of CNTs in $U$ lon batterles dates back to 2001. However, all those initial efforts ${ }^{4-6}$ could only obtain a maximum capacity of 280 mAh $\mathrm{g}^{-1}$, much below the theoretical $\mathrm{ca}^{-}$ pacity of graphite. Though a few recent efforts have shown higher attainable reversible capacitles using single-walled nanotubes (SWNTs), the processing techniques involve defect creation by etching or ball milling, use addittives in electrolyte, and apply CNT paste (with organic binder) as anode. ${ }^{\text {TA }}$ However, like graphite, CNTS showed good stablity in the long run. ${ }^{\circ} \mathrm{Re}$ searchers also used other allotropes of carbon, graphene and fullerene and composItes of them, as anodic replacement of graphite in $u$ ion cells. ${ }^{10}$ The best capacity obtained from these materials is $784 \mathrm{mAh}$ $\mathrm{g}^{-1}$ at a low current rate of $0.13 \mathrm{C}$ (for graphene-fullerene composite), but with a capacity fading with cycles. Among other materlals, $\mathrm{Sl}$ and $\mathrm{SnO}_{2}$ attracted much attention because of their very high theoretical spectic capacitles, 4200 and 782 mAh g $^{-1}$ respectively, ${ }^{11,12}$ but both these materials suffer from the limitation that during $u$ ion Intercalation and deintercalation, they experlence huge (300-400\%) expansion and contraction, resulting in pulverization and capactty loss in a high number of cycles. Though it was shown that an efficlent design of their nanostructures can generate 


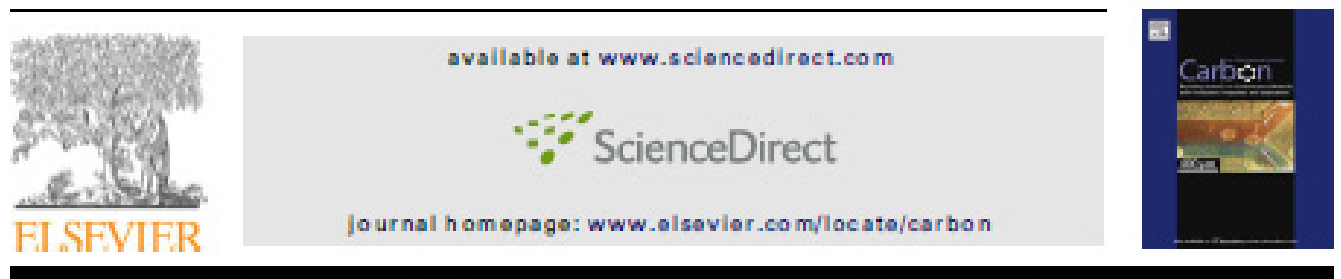

\title{
Enhanced field emission from multi-walled carbon nanotubes grown on pure copper substrate
}

\author{
Indranil Lahiri ${ }^{a}$, Raghunandan Seelaboyina ${ }^{a}$, Jun Y. Hwang ${ }^{b}$, Raj Banerjee ${ }^{b}$, \\ Wonbong Choi ${ }^{a}$,
}

"Nanomaterials and Devices Laboretory, Deparonent of Mechanical and Materials Engineering, Florida Intemational University. Miami, FL 33174, USA

"Departmont of Materials Science and Enginaring, Univeraity of North Texas, Denton, TX 76203, USA

\section{ARTICLEINFO}

Artick kiatory

Recelved 13 May 2009

Acceptad 19 Nowember 2009

Novalable online 28 December 2009

\section{A B S T R ACT}

An efficient CNT baged feld emitwr was prepaed on a Cu subatrate waing thermal chem. ical vapor deposition. Pld emiasion characteriation of the emitter showed a very low turn on feld, high emist on current, long tome stability and good redistrnce to degadation in high-feld, long tome exponus. These superior feld emistion chancwriatcs are atrb. uted to a lowe contact banier and higher conductivity of the subatrate. High resolution tansmiasion electron microsco pe analysia showed the presence of a conducting phase, $\mathrm{TC}$, at the meal CNT inverface, providing a bow contact wastance barrier.

o 2009 Flavewer Ltd. All tights reserved.
1.

Introduction

Cold field emitters have subatantially replaced thermionic emitters in almost all vacum electronic devios [1,2] like electron microsopes [3], X-ray tibes [4], microwave power amplifiers 15], field emission dimlays 16]. Among the field emitters, carbon nanotibe (CNT) array emitter has attracted most attention due to its fine tips, high aspect ratio, good chemical resistanos, efficient thermal onductivity and exceptional mechanical strength [7-10]. Due to it potential applien tions in semiconductor industries. CNTs are corven. tionally grown on Si wafers. However, Si is not the best muit able substrate matwial for applications where high electrical and thermal conductivity are required. Thus it is important to grow CNTs on suitable metallic subs tra tws without compromising the properties required for the intended applica tion. Moreover, minimization of the contact resistance between a thin metal film and the CNTs has remained a ma. jor isure in nano-electronics [11]. Apart from geometrical fac. tors, contact resistance depends mostly on alignment of Fermi energy levels of CNT and the subatrate Since, MWCNTs are predominantly metallic, thus a metallic substrate is ex pected to have least contact resiatance. Talapatra et al. [12] have directly grown COIIs on Inonel mibstrate and stressed upon the inmediate noed of anthesizing CVTs on high conductivity metal surfaces, for application in interconnects, super capadion and field emitters. The present paper focuses on the field emitter application.

The most important requirement of a commerdal field emitter device are low tum on field, high emiarion current, and good stability of emistion. While an ohmic contact enaures easy electron transport and thus low turn on field, a well-developed CNT structure supports high emission current. Howerer, emistion stability is affected by many parameters such as low substrate-CNT barrier (to ensure un-internupted electon transort from substrate to CNT structure), high thermal conductivity of the subatrate to ex. tract ary heat that is generated due to contact resistance (b) minimize debonding of CNTs from subatrate), presence of gas contamimants on CVI surface (most of which incresses work function, hinders continuous tunneling of electrons from the emitter suface, thus rectucing emissivity). poor

\footnotetext{
- Correponding actha: Fax +1 3053481932

E.mad address chotwetru adu (WB. Cho i)

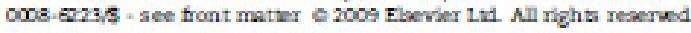

doil10. D16/) carbon 2009.11 .064
} 
Gte this: J. Mater. Chem, 2011, 21, 7289

\title{
Synthesis and characterization of self-organized multilayered graphene-carbon nanotube hybrid films
}

\author{
and Wonbong Choi twe \\ Received 20th Januany 2011, Accepted 9th March 2011 \\ DOE: $10.1039 \mathrm{kcl}$ jm1031 6d \\ We report the synthesis and characterization of a sdf-organized graphene-carbon nanotube (CNT) \\ hybrid film ansisting of multilaye graphene (MLG) supported by vertical CNTs $\propto \mathrm{\alpha}$ a Si substrate. The \\ hybrid film shows an interesting structure of graphene in connecting to vertical CNTs while \\ maintaining ohmic contact at its junction. The in situ fornation of a CNT-graphene heterojunction is \\ wnfirmed by high resolution transmission dectron microcopy (HR TEM). The as-obtained film shows \\ a hinar rdationship for electrical conductanow with significantly low resis tance var ying from $100-400 \Omega$ \\ on Si substrate. This hybrid film is inverted and transferred to flexible substrates for its application in \\ flexible electronics, demonstrating an observable variation of electrical conductivity for both tansion \\ and compression. Furthermore, both turn-on fiekd and total anission current were found to depend \\ strongly on the bending radius of the film and were found to vary in ranges of 0.8-3.1 $\mathrm{v} \mu \mathrm{m}^{-1}$ and \\ $4.2-0.4 \mathrm{~mA}$, respectively.
}

Santanu Das, " Raghunandan Seekboyina, ${ }^{a}$ Ved Verma, , Indranil Lahiri, ${ }^{e}$ Jun Yeon Hwang, ${ }^{b}$ Rajarshi Banerjee ${ }^{b}$

\section{Introduction}

Carbon manomaterials have ben studied over the last 2 decades with great attention for their fasibility in future applications due to their exaptional properties like high current carrying capacity, ballistic charge transport, high themal conductivity, chemical inertness, and high mechanical strength ${ }^{1-t h}$ Fulkene (OD), Garbon nanotube (CNT) (1D) and graphane (2D) are the carbon nanostructures most widely investigated in order to explore their novel properties. Apart from their individual properties, hybrid properties are also studied and applications have ben proposed based on fulkenes-CNT, graphenefulkene and graphene-CNT due to the significant technological importance of the hybrid structure. ${ }^{11,2 e s}$. The 1D form, carbon manotube, has ben stucied rigorously for last 2 deades, but has limited applications due the difficulties in manufacturing it ondemand with uniform electrical and physical properties. ${ }^{19-20}$

Since the firstreport on isolation of single byer graphene sheet in 2004, scaling up of graphene is a major concem which is not only for its scientific interest but also for its impact on the feasi bility of different tachnological applications. ${ }^{60}$ So far graphene has ben synthesined in either on SiC or metal substrates, and transfarred to other fkxible substrates afterwards bo beal for fexible electronics and solar all electrodes. ${ }^{14-17}$ The res ul ts due to the exceptional properties of graphene, even in the device proto

- Departmar of Mechavioal and Materids Englnaging. Flarida

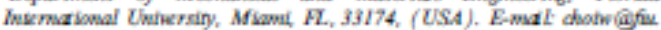

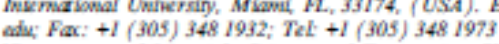

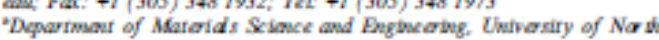
Texas, Datrow, TX, 70203, USA type stage, has led to danonstration of various devices in a wide range of areas including fiekd emission (FE) cathode, sensors, electronics, solar cells, Li-ion batteries and so on. ${ }^{2-69-1221}$

In addition to the appications based on either graphene or CNT, the graphene-CNT hy brid structure is highly beneficial for microdedronics applications as it can incorporate the advantages from properties of graphene and CNTs in the planar and axial directions, respectively. In particular, direct transfer of CNTs onto a polymer matrix suffers from lack of a homoge. noous bottom contact for the CNT layers, as polymer substrates arehigh resis tance in nature. In this context, the hybrid structure will be in high demand as it coukd provide a uniform conducting graphene byer which woukd be beneficial for applying on any type of polymer substrate. Furthemnore, the themnal condustivity of a multiwall manotube (MWCNT) is $\sim 3000 \mathrm{~W} \mathrm{mK}^{-1}$ which reduces by several orders of magritude even to $20 \mathrm{~W} \mathrm{mK}^{-1}$ or lower when MWCNTs are in the form of mat or forest structures. ${ }^{12,11}$ The primary rasson for the reduction in themal conductivity is due to the discontinuitis in the homogeneous bottom ontacts in all the forest CNTs. ${ }^{12}$ Similarly, eloctrical conductivity is also affected due to the non-uniform contact between all the CNTs in a mat or forest structure CNT film over any substrate. ${ }^{1,121 k a s}$ al Some of these shortomings can be addressed by the unique graphene-carbon manotube hy trid film, consisting of CNTs connacted perpandiaularly to graphene byers. Recently, Lee et al $^{\text {2h }}$ reportal an interesting approach to grow vertically aligred MWCNTs on rafuced graphene oxide platelets and proposed its applicability as a flexible fidd emission device. However, the reduad graphene oxide involves bot of issus like, low conductivity, kss uniformity, and high 


\title{
Controlled Growth of Single-Walled Carbon Nanotubes for Unique Nanodevices
}

\author{
Jun Huang ${ }^{1}$, Unjeong Kim², Bei Wang ${ }^{3}$, Indranil Lahiri', Eunhong Lee ${ }^{2}$, \\ Peter C. Eklund ${ }^{3,4}$, and Wonbong Choi ${ }^{1, *}$ \\ 'Department of Mechanical and Matenals Engineening, Florida International Untversity, Miami, FL, 33174 USA \\ ${ }^{2}$ Samsung Advanced Institute of Technology (SAIT), Yongin, Gyeonggi-do, 446-712 Republic of Korea \\ ${ }^{3}$ Department of Physics, The Pennsylvania State University, University Park, PA, 16802 USA \\ ${ }^{4}$ Department of Materials Science \& Engineering and Materials Research Institute, \\ The Pennsyivania State University, University Park, PA, 16802 USA
}

The controlled growth of bent and horizontally aligned single-walled carbon nanotubes (SWNTs) is demonstrated in this study. The bent SWNTs growth is attributed to the interaction between van der Waals force with substrate and aerodynamic force from gas flow. The curvature of bent SWNTs can be tailored by adjusting the angle between gas flow and step-edge direction. Electrical characterization shows that the one-dimensional resistivity of bent SWNTs is correlated with the curvature, which is due to strain induced energy bandgap variation. Additionally, a downshift of $10 \mathrm{~cm}^{-1}$ in G-band is found at curved part by Raman analysis, which may be resulted from the bending induced carbon-carbon bond variation. In addition, horizontally allgned SWNTs and crossbar SWNTs were demonstrated. To prove the possibility of integrating the SWNTs heving controllable morphology in carbon nanotube based electronics, an inverter with a gain of 2 was built on an individual horizontally aligned carbon nanotube.

Keywords: Single-Walled Carbon Nanotube, Controlled Growth, Bending, Horizontally Aligned, Electrical Property, Inwerter.

\section{INTRODUCTION}

The unique electrical and mechanical properties of singlewalled carbon nanotubes (SWNTs) make them one of the most promising candidates for next generation nanoelectronics. ${ }^{1}$ For better utilization of the electrical properties of SWNTs, it is crucial to control their morphologies (e.g., straight, junction, coiled) and growth direction (e.g. vertical, horizontal). The growth of SWNTs with the controlled morphologies and functionalities may open a new way to interesting nanoelectronic circuits and nanoelectromechanical systems (NEMS). In this report, we demonstrate novel SWNTs devices based on different morphologies, i.e., bent and horizontally aligned SWNTs.

In the previous reports, horizontally aligned SWNT growth has been demonstrated by application of electric field during growth, ${ }^{2}$ using "fast-heating" method, ultra low gas flow method, ${ }^{4}$ and utilizing certain types of singlecrystal substrates, including quart $z^{5-9}$ and sapphire. ${ }^{0-14}$ Complex geometries, such as crossbar structure, have been

\footnotetext{
-Autbor to whom correspondence should be addressed.
}

achieved by multi-step transfer of aligned SWNTs onto other substrates ${ }^{9}$ or by applying electric-field during nanotube growth. ${ }^{12}$ However, direct growth of SWNTs in the complex geometries still remains a challenging task. To fully appreciate SWNTs in nanoelectronic circuit or nanoelectromechanical systems (NEMS), it may be necessary to grow SWNTs having different morphologies and functionalities. Very recently, SWNTs with multiple bends (so called nanotube serpentines) have been grown by catalytic chemical vapor deposition and no appreciable current drops were found along the nanotube serpentines by electrostatic force microscopy. ${ }^{15}$ The present study, however, show that the curvature of bent SWNTs plays an important role in electron transport, which may be attributed to the introduction of topological defects, heterojunctions and local strain at the bends. The existence of negative curvature fullerene based units in bent SWNTs necessitates the presence of topological defects (in the form of pentagons, heptagons and octagons) at the junction regions to maintain a low energy $s p^{2}$ configuration. ${ }^{16}$ Presence of these intrinsic defects can modify chirality of bent SWNTs across the junctions and thus hetero-junctions

$1533-486072011 / 11 / 2622003$ doi: $10.11665 / \mathrm{gn} .2011 .3156$ 


\title{
Tuning Electrical Conductance of Serpentine Single-Walled Carbon Nanotubes
}

\author{
By Jun Huang Bei Wang, Indranil Lahiri, Awnish K. Gupta, Peter C. Eklund, \\ and Wonbong Choi*
}

Changes in resistivity of serpentine single-walled carbon nanotubes are presented as a function of bending radius, $r_{b}$, in the range of $100-2000 \mathrm{~nm}$. Resistivity $(\rho)$ is observed to increase with curvature $\left(1 / r_{b}\right)$, which is consistent with theoretical speculation on strain-induced bandgap increment. Furthermore, a sharp bend $\left(r_{b}<50 \mathrm{~nm}\right)$ in the nanotubes results in a drastic change in the field-effect behavior, i.e., from ambipolar to $p$ type across the bend. Local Raman spectra show that the G-band Raman frequencies shift along the curvature, which may be attributed to local deformation and broken cylindrical symmetry in the nanotubes. The results suggest the possibility to tune the electrical properties by bending nanotubes and to build an allnanotube device by modulating the structure of the same tube.

\section{Introduction}

The unique electrical and mechanical properties of single walled carbon nanotubes (SWNTs) make them one of the most promising candidates for nanoelectronics. [1.] Although SWNTs have been studied for more than a decade, large-scale integration of the tubes into micro- or macroscale systems has not yet been achieved. One of the major bottlenecks is the difficulty to efficiently control the namotube electronic properties, which is directly related to diameter and wrapping indices $(n, m)$ of SWNTs. II] To utilize SWNTs as different electronic components (e.g., resistor or transistor), it is critical to tailor the electrical properties of the SWNIs. One of the simplest approaches, in

[*] Dr. J. Huang I. Lahiri, Prof.W. B. Choi

Department of Mechanical and Materials Engineering

Florida International University

Mami, FL 33174, USA

B. Wang A. K. Gupta

Department of Physics

The Penngyivania State University

University Park, PA 16802, USA

Prof. P. C. Eklundil

Department of Physics

Department of Materials Science \& Engineering and Materials

Department of M

Pesearch Institute

University Park, PA 16802, USA

lii] Prof. Eluund passed away in Aug. 2009

DOl: $10.1002 / \mathrm{adfm} .201000957$ terms of ease of operation, is to control the conductance of individual SWNTs by mod. ulation of the morphology via mechanical perturbation. For instance, atomic force microscopy (AFM) tips have been used to introduce local defects in SWNT strueture $]^{3]}$ A mechanical perturbation can induce defect sites into the SWNT structure, which results in a drop of conductivity due to increased density of scattering centers. Moreover, the theoretical speculation of bandgap variation in the bent part can play an important role. 1.51 How. ever, synthesis of locally stressed SWNT structure, in the growth stage itself has remained as a theoretical possibility for a long time. Recently, self-organized SWNT serpentine objects were reported to be synthesized by controlling the combined forces of surface adhesion and aerodynamic drag ${ }^{[67]}$ By uti. lizing the parallel segments of an individual serpentine tube, Jeon et al. ${ }^{3 / 1}$ and Yao et al ${ }^{19}$ have achieved high current output from bent nanotube devices, which is promising for future high-current carbon-nanotube-based nanoelectronic devices. Until now, however, in-depth study to correlate the curved part of these serpentine tubes with their electrical conductance has not been performed. Our previous result demonstrated that an SWNT segment with sharp curvature has higher resistance than a smooth one.? The resistance change may be attributed to the induced strain where the C-C bonds on the inner and outer sides of the bend are put under compressive and tensile strain, respectively. It was also reported that a strain of $3.4 \%$, imposed via AFM manipulation, can reduce the conductance by two orders of magnitude. $\left.\right|^{3]}$ Uniaxial strain in metallic (m) SWNTs has been theoretically proposed to open small gaps at the Fermi level $\left(E_{E}\right)$, while in semiconducting (s) SWNTs, it induces significant change in the energy bandgap, i.e., $\Delta E_{g}$

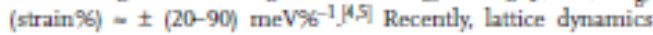
calculations have predicted that G-band components of Raman spectrum change their position and intensity due to broken symmetry in bent SWNIs, when the curvature of the bend is high. ${ }^{10}$ Very sharp curvature can produce kinks in the tube wall and possibly induce a perturbation of the $\mathrm{sp}^{2}$ structure of the SWNT and a noticeable shift in Raman spectrum. Although controlled formation of serpentine SWNTs has been reported by our group and others, systematic study of curvature effect on electrical property has not been reported yet. Here, we present experimental studies of the resistivity of serpentine 


\title{
Large-area graphene on polymer film for flexible and transparent anode in field emission device
}

\author{
Ved Prakash Verma, Santanu Das, Indranll Lahin, and Wonbong Chol ${ }^{\mathrm{a}}$ ) \\ Department of Mechanical and Materials Engineering, Florida International University, Miami, \\ Florida 33174, USA
}

(Received 25 March 2010; accepted 28 April 2010; published online 20 May 2010)

\begin{abstract}
We present the fabrication and electrical characterization of large graphene structure on polyethylene terephthalate (PET) flexible substrate. Graphene film was grown on $\mathrm{Cu}$ foil by thermal chemical vapor deposition and transferred to PET by using hot press lamination. The graphene/PET film shows high quality, flexible transparent conductive structure with unique electrical-mechanical properties; $\sim 88.80 \%$ light transmittance and $\sim 1.1742 \mathrm{k} \Omega / \mathrm{sq}$ sheet resistance. We demonstrate application of graphene/PET film as flexible and transparent electrode for field emission displays. Our proposed techniques can be tailored for any flexible substrate and large scale production, which could open up exciting device applications in foldable electronics. 2010 American Institute of Physics. [doi:10.1063/1.3431630]
\end{abstract}

Graphene is a two-dimensional (2D) carbon material having unique band structure and outstanding thermal, mechanical, and electrical properties. ${ }^{1-3}$ Some of the potential applications of graphene are for sensors, transistors, supercapacitors, solar cells, and flexible displays. ${ }^{48}$ It is well known that graphene has high mechanical strength with flexibility, high transmittance, and high electron mobility. ${ }^{8,9}$ These properties make graphene an emerging alternate for transparent conductive metal oxides electrodes, in particular, indium tin oxide (ITO) which contains indium as a costly and scarce element.

In order to make a transparent conductive graphene film, most of the researchers have used liquid solution of graphene flakes (obtained by reducing graphene oxide flakes) for deposition of transparent conductive film. ${ }^{7,10}$ Recently, Yamaguchi et $a L^{11}$ has deposited chemically derived graphene solution on flexible substrate for large area transparent flexible electrode which contains 2 to 30 layers of graphene. Application of graphene in flexible electronics will need synthesis of continuous graphene film on substrates and transfer it to polymeric substrate in large scale. Li ef $a l^{12}$ has grown high quality, predominately monolayer graphene films on copper foils by chemical vapor deposition (CVD) method. Kim et al. ${ }^{8}$ have demonstrated two different techniques (stamping and scooping) for transferring graphene from nickel substrate to other arbitrary substrates. These techniques are not effective for industrial application which will require low cost, high quality, and large area production of graphene flexible electrodes.

Here we present a direct and effective method for synthesis of large graphene film on copper foils and transferring it to polyethylene terephthalate (PET) flexible substrate by hot press lamination process. This method provides an effective way to handle large area of graphene film with minimal physical damage to it. The resulting graphene polymer film is flexible and remains conductive under high tensile strains. The application of this graphene film as flexible transparent conductive anode has been demonstrated in carton nanotube (CNT) field emission devices (FEDs).

"Electrunic mail: choiw G fiu ede.
Graphene film was synthesized by CVD of hydrocarbon on copper foil. Commercially available, cold rolled $\mathrm{Cu}$ foil of 50 to $200 \mu \mathrm{m}$ thickness and large area $(6 \mathrm{~cm}$ width and $15 \mathrm{~cm}$ length) was first annealed at $1000^{\circ} \mathrm{C}$ for $1 \mathrm{~h}$ under $\mathrm{Ar}$ atmosphere. After annealing, $\mathrm{Cu}$ foil was acid-treated for 10 min using $1 \mathrm{M}$ acetic acid at $60^{\circ} \mathrm{C}$. This acid treatment helps in removing oxide layer generated at the Cu foil surface during annealing process. Copper foils were thoroughly washed with de-ionized water and dried at the ambient conditions. Graphene films were grown on copper foils in a similar way to the previously reported CVD process. ${ }^{12}$ In short, graphene on the $\mathrm{Cu}$ foil was synthesized at $1000^{\circ} \mathrm{C}$ and 1 atm pressure, using a 5 min flow of $\mathrm{CH}_{4}$ and $\mathrm{H}_{2}$ gases in 1:4 ratios. After graphene growth, the foil was cooled down to room temperature before being taken out from the furnace. Graphene growth on $\mathrm{Cu}$ foil has been reported as a surface-catalyzed process which indicates that $\mathrm{Cu}$ act as catalyst for CVD of graphene. A detailed discussion about growth mechanism of graphene formation on copper foil has been presented elsewhere. ${ }^{12}$

We have used hot press lamination and chemical etching process for transferring graphene grown over the $\mathrm{Cu}$ foils to the transparent flexible substrates. Figure 1(a) shows flow diagram of graphene transfer technique. $\mathrm{Cu}$ foils with graphene were hot press rolled with a transparent flexible PET film having thickness $\sim 50 \mu \mathrm{m}$. For complete removal of $\mathrm{Cu}$ from the graphene and laminated film, we have used concentrated $\mathrm{FeCl}_{3}$ solution. Laminated polymer film with graphene and $\mathrm{Cu}$ foil undemeath was floated over the $\mathrm{FeCl}_{3}$ acid bath at room temperature. After $\mathbf{4 0}$ min of etching process $\mathrm{Cu}$ was completely dissolved into the solution leaving graphene film with the PET substrate. This transparent flexible film was then thoroughly washed with de-ionized water and dried in air at room temperature. Figure l(b) demonstrates a flexible, transparent graphene film with diagonal length of $\sim 16 \mathrm{~cm}$. This hot press lamination process provides a very adherent graphene film on the flexible substrate which can be deformed easily into various geometries [Fig. 1(c)] without damaging the film.

Characterization of graphene over $\mathrm{Cu}$ and flexible substrate were done by Raman spectroscopy. The Raman spec- 


\title{
Carbon-nanotube-embedded novel three-dimensional alumina microchannel cold cathodes for high electron emission
}

\author{
Raghunandan Seelaboyina, Indranil Lahiri and Wonbong Choi ${ }^{1}$ \\ Nanomalerials and Devioes Lab, Mechanical and Materials Engineering Department, \\ Forida Internasional University, Miami, FL. 33174, USA \\ E-maik choiw 9 fruedu \\ Recetved 16 December 2009 , in final form 28 January 2010 \\ Published 16 March 2010 \\ Online at stacks.iop.org/ $\mathrm{Nano} / 21 / 145206$

\section{Abstract} \\ The present work describes a comprehensive design and scalable micro-fabrication technique \\ for the production of novel 3D cathodes, consisting of chemical-vapor-deposition-grown \\ high-density multi-wall carbon nanotubes along the walls of alumina microchannels. Under \\ high DC and AC electric nelds, the 3D cathodes displayed signincantly high and moderately \\ stable emission current of $\sim 5.25$ and $\sim 14 \mathrm{mLA}$, respectively. The inherent advantages of the 3D \\ microchannel geometry for cold cathodes are higher emitter area, less ion bombardment and \\ robustness under high voltage conditions. The 3D cathodes are envisioned to offer an entirely \\ new class of miniature electron sources for high current vacuum microelectronics. \\ (Some ngures in this article are in colour only in the electronic version)
}

\section{Introduction}

Feld emission cathodes baed on carbon nanotubes (CNTs) are innovative electron sources that can potentially replace themionic cathodes. The extraordinary neld emission (FE) properties of CNTs arise due to their unique structure, and remarkable mechanical, electrical and chemical properties [1-5]. Furthermore, the large aspect ratio and nanodimensional apex of CNTs has resulted in electron emission at exceptionally low threshold electric nelds $\left(F_{\text {th }}\right)$ of $0.5-2 \mathrm{v} \mu \mathrm{m}^{-1}[1-10]$. Emission current density of $\sim 10 \mathrm{~mA} \mathrm{~cm}{ }^{-2}$ can be achieved from CNT emitters at an $F_{\mathrm{h}}$, of the order of $1-10 \mathrm{~V} \mu \mathrm{m}^{-1}[4]$, which is relatively low, compared to several other emitters, such as diamond and Spindt-type tips $[4,6,7]$. All the aforementioned properties make CNTs suitable for several technological applications. Few of the demonstraled prototype devices based on FE properties of CNTs include feid emission displays [9], electron sources for high power microwave (HPM) cathodes $[1,3]$ and miniature $x$-ray sources [10]. Despite numerous ploneering research reports on CNT FE properties, several challenges including long-term emission stability with high emission current, emission uniformity and

${ }^{1}$ Nuthor to whom any com:pondence should be adiresed

$095-443410145206+0053000$ methods for the emcient production of CNT cathodes still exist [4].

Emission current from CNT cold cathodes has been suggested to increas, by enhancing the number of CNT emitters present in a specinc area, provided the majority of the emitters participate in the electron emission process $[4,5]$. For any cold cathode, in addition to higher emission curnent, stable operation in the range of a fow m.A to $\mathrm{A}$ in highelectric Deld conditions [1-5] is also essential. To achieve such a high magnitude of emisslon cuntent, electron sources in HPM and other high current applications have to be operated at high electric nelds, which may caus debilitating effects such as ion bombardment and arcing [4]. Dwe to these phenomena, performance of cold cathodes can be permanently impaired To address these issues, in this report for the first time ever we propose a novel cold cathode concept for moderately high, uniform and stable electron emission. As shown in figures 1 (a) and 2(a)-(c), the novel cathode consists of chemical-vapor-deposition (CVD)-grown high-density multwall carbon nanotubes (MWNTs) along the walls of threedimensional (3D) alumina $\left(\mathrm{Al}_{2} \mathrm{O}_{3}\right)$ microchannels. The inherent atvantage of 3D cathodes, compared to conventional planar two-dimensional (2D) cathodes, is enhanced emitter density in a specinc area and robusiness at high voltage conditions To date, the primary geometry of the substrate on 


\title{
Synthesis of Graphene and Its Applications: A Review
}

\author{
Wonbong Choi, ${ }^{1,+}$ Indranil Lahiri, ${ }^{1}$ Raghunandan Seelaboyina, ${ }^{1}$ \\ and Yong Soo Kang ${ }^{2}$ \\ ${ }^{1}$ Nanomaterials and Devices Laboratory, Florida International Universily, Miami, Florida, USA \\ ${ }^{2}$ Department of Energy Engineering, Hanyang University, Seoul, Korea
}

\begin{abstract}
Graphene, one-atom-thick planar sheet of carbon atoms densely packed in a honeycomb crystal lattice, has grabbed appreciable attention due to its exceptional electronic and optoelectronic properties. The reported properties and applications of this two-dimensional form of carbon structure have opened up new opportunities for the future devices and systems. Although graphene is known as one of the best electronic materiak, synthesizing single sheet of graphene has been less explored. This review article aims to present an overview of the advancement of research in graphene, in the area of synthesis, properties and applications, such as field emission, sensors, electronics, and energy. Wherever applicable, the limitations of present knowledgebase and future research directions have also been highlighted.
\end{abstract}

Keywords electronic propertics, growth techniques, scnsors, tnansistors, electrodes, rechargeable batiery

Table of Contents

1. INTRODUCTION

2. PROPERTIES OF GRAPHENE

2.1. Properties of Single- and Bi-layer Graphene

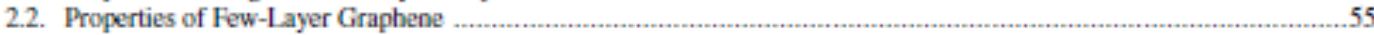

3. GRAPHENE SYNTHESIS METHODS

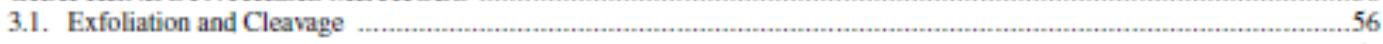

3.2. Thermal Chemical Vapor Deposition Techniques …..................................................................................57

3.3. Plasma Enhanced Chemical Vapor Deposition Techniques .........................................................................58

3.4. Other Processing Routes …n......58

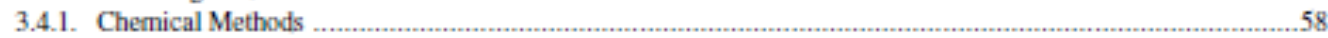

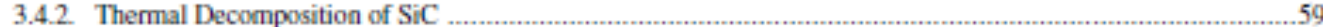

3.4.3. Thermal Decomposition on Other Substrates .............................................................................60

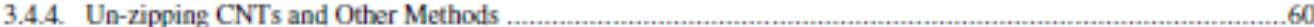

4. APPLICATIONS OF GRAPHENE

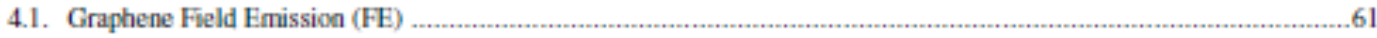

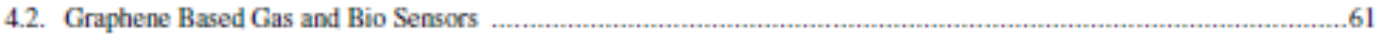

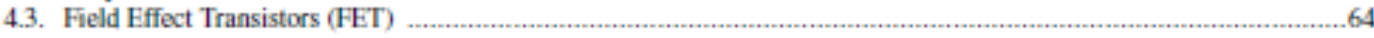

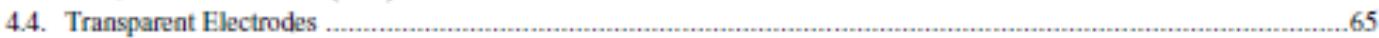

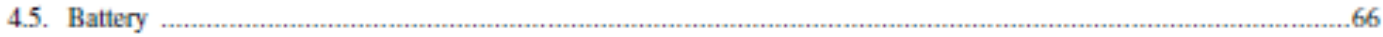

5. SUMMARY

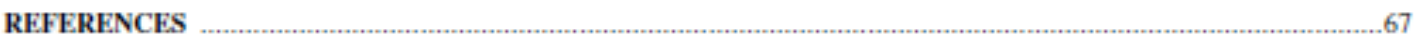

"E-mail: choiw@ fiu.edu 
VITA

INDRANIL LAHIRI

August 2, 1975

Aug. 1994 - Jun. 1998

Aug. 1998 - Jun. 2000

Jan. 2008 - Sep. 2011
: Born, Kolkata, India

: Bachelor of Engineering (B.E) in Metallurgical Engineering, Bengal Engineering College, Shibpur, India (Second position in class of 1998)

: Master of Technology (M. Tech) in Metallurgical and Materials Engineering, Indian Institute of Technology, Kanpur, India

: Doctoral Candidate (Ph.D) in Florida International University, Miami, USA (GPA - 4.0/4.0)
PROFESSIONAL EXPERIENCE

May 2000 - Apr. 2005

May 2005 - Jun. 2007
: Scientist, Non Ferrous Materials Technology

Development Centre, Hyderabad, India

: Senior Scientist, Non Ferrous Materials

Technology Development Centre, Hyderabad, India

\section{ACADEMIC AWARDS AND HONORS}

1. Recipient of Dissertation Year Fellowship (DYF) by University Graduate School, FIU, Spring-Fall, 2011.

2. Recipient of Dissertation Evidence Acquisition (DEA) Fellowship by University Graduate School, FIU, Summer-Fall, 2010.

3. Won TMS Student Best Paper Contest 2011 - Graduate Level $-2^{\text {nd }}$ Place.

4. Awarded Second prize in "The Best Junior Researcher Awards in 2011 Nanomaterials Symposium", TMS 2011.

5. Selected as Student Board Member, ASM International - Board of Trustees, 2010-11.

\section{PUBLICATIONS AND PRESENTATIONS}

Journals (Published/Accepted)

1. Indranil Lahiri, Seung-Min Oh, Jun Y. Hwang, Chiwon Kang, Hyeongtag Jeon, Rajarshi Banerjee, Yang-Kook Sun, Wonbong Choi, Ultrathin alumina coated carbon nanotubes as negative electrodes for high capacity and safe Li-ion battery, Journal of Materials Chemistry (DOI: 10.1039/C1JM1 1474C). (Impact Factor: 5.099)

2. Indranil Lahiri, Wonbong Choi, Interface control: A modified rooting technique for enhancing field emission from multiwall carbon nanotube based bulk emitters, Acta Materialia, 59, 2011, 5411-5421. (Impact Factor: 3.781) 
3. Indranil Lahiri*, Debrupa Lahiri*, Sungho Jin, Arvind Agarwal, Wonbong Choi, Carbon Nanotubes: How strong is their bond with the substrate?, ACS Nano, 5(2), 2011, 780-787. (Impact Factor: 9.855). * Co-first authors

4. Indranil Lahiri, Ved Prakash Verma, Wonbong Choi, An all-graphene based transparent and flexible field emission device, Carbon, 49 (5), 2011, 1614-1619. (Impact Factor: 4.893)

5. Indranil Lahiri, Santanu Das, Chiwon Kang, Wonbong Choi, Application of carbon nanostructures - Energy to electronics, JOM, 63(6), 2011, 70-76. (Impact Factor: $1.175)$

6. Indranil Lahiri, Sung-Woo Oh, Jun Y. Hwang, Sungjin Cho, Yang K. Sun, Rajarshi Banerjee, Wonbong Choi, High capacity and excellent stability of lithium ion battery anode using interface-controlled binder-free multiwall carbon nanotubes grown on copper, ACS Nano, 4(6), 2010, 3440-3446. (Impact Factor: 9.855)

7. Indranil Lahiri, Raghunandan Seelaboyina, Jun Y Hwang, Raj Banerjee, Wonbong Choi, Enhanced field emission from multi-walled carbon nanotubes grown on pure copper substrate, Carbon, 48 (5), 2010, 1531-1538. (Impact Factor: 4.893)

8. Jun Huang, Bei Wang, Indranil Lahiri, Awnish K. Gupta, Peter C. Eklund, Wonbong Choi, Effect of bending on the resistivity and Raman spectrum of single-walled carbon nanotubes, Advanced Functional Materials, 20, 2010, 4388-4393. (Impact Factor: 8.486)

(Listed 8 out of 22 published journal articles and 4 peer-reviewed symposium proceedings)

\section{Book Chapters}

1. Indranil Lahiri, Wonbong Choi, Field emission and graphene: An overview of current status, In "Graphene: Synthesis and applications" Ed. W. Choi, J.-W. Lee, CRC Press (Boca Raton, USA), October 2011 (expected publication date), ISBN: 9781439861875.

2. Indranil Lahiri, Wonbong Choi, Graphene and graphene based materials in solar cell application, In "Graphene: Synthesis and applications" Ed. W. Choi, J.-W. Lee, CRC Press (Boca Raton, USA), October 2011 (expected publication date), ISBN: 9781439861875.

Patent

1. W.B. Choi, S.J. Cho, I. Lahiri, High efficiency lithium ion battery anode using interface-controlled binder-free carbon nanotubes grown on metal/alloy substrates, US Provisional Patent Appl. No. 61/222,481 (Filed on July 2, 2009).

\section{Conference Presentations}

28 technical presentations in international conferences, including major conferences like TMS Annual Meeting, MRS Meetings, ECS Meetings, MS\&T etc. 